\title{
Deciphering mitochondrial and molecular pathology in Type 2 Diabetes Mellitus
}

Citation for published version (APA):

van Tienen, F. H. J. (2011). Deciphering mitochondrial and molecular pathology in Type 2 Diabetes Mellitus. [Doctoral Thesis, Maastricht University]. Universiteit Maastricht. https://doi.org/10.26481/dis.20110204ft

Document status and date:

Published: 01/01/2011

DOI:

10.26481/dis.20110204ft

Document Version:

Publisher's PDF, also known as Version of record

\section{Please check the document version of this publication:}

- A submitted manuscript is the version of the article upon submission and before peer-review. There can be important differences between the submitted version and the official published version of record. People interested in the research are advised to contact the author for the final version of the publication, or visit the DOI to the publisher's website.

- The final author version and the galley proof are versions of the publication after peer review.

- The final published version features the final layout of the paper including the volume, issue and page numbers.

Link to publication

\footnotetext{
General rights rights.

- You may freely distribute the URL identifying the publication in the public portal. please follow below link for the End User Agreement:

www.umlib.nl/taverne-license

Take down policy

If you believe that this document breaches copyright please contact us at:

repository@maastrichtuniversity.nl

providing details and we will investigate your claim.
}

Copyright and moral rights for the publications made accessible in the public portal are retained by the authors and/or other copyright owners and it is a condition of accessing publications that users recognise and abide by the legal requirements associated with these

- Users may download and print one copy of any publication from the public portal for the purpose of private study or research.

- You may not further distribute the material or use it for any profit-making activity or commercial gain

If the publication is distributed under the terms of Article $25 \mathrm{fa}$ of the Dutch Copyright Act, indicated by the "Taverne" license above, 


\section{Deciphering mitochondrial and molecular pathology in Type 2 Diabetes Mellitus}




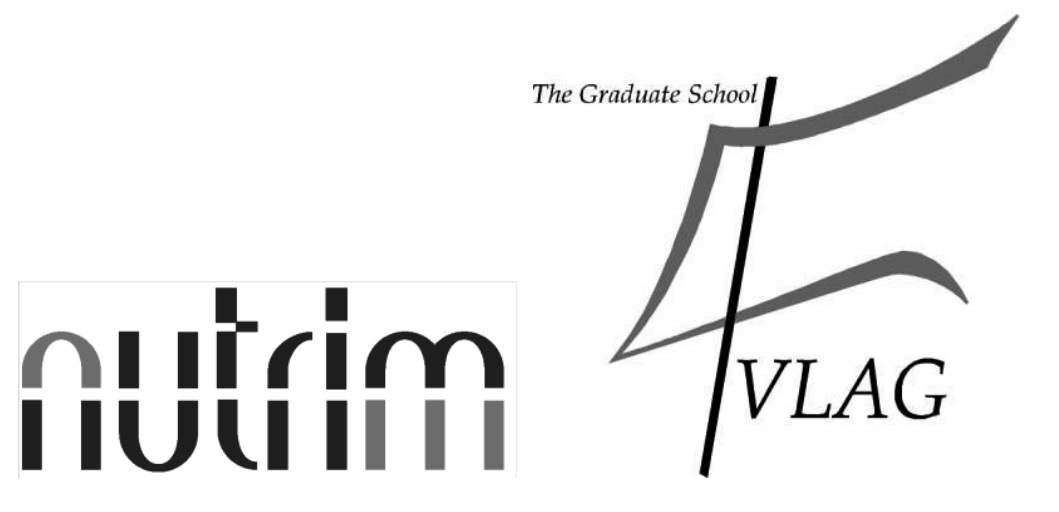

The study presented in this thesis was performed within NUTRIM School for Nutrition, Toxicology and Metabolism which participates in the Graduate School VLAG (Food Technology, Agrobiotechnology, Nutrition and Health Sciences), accredited by the Royal Netherlands Academy of Arts and Sciences.

(c) Florence H. J. van Tienen, Obbicht 2010

ISBN 978-90-8891-227-6

Cover design: $\quad$ Florence van Tienen and BOXPress

Printed by: Proefschriftmaken.nl || Printyourthesis.com

Published by: Uitgeverij BOXPress, Oisterwijk 


\section{Deciphering mitochondrial and molecular pathology in Type 2 Diabetes Mellitus}

\section{Proefschrift}

ter verkrijging van de graad van doctor aan de Universiteit Maastricht, op gezag van de Rector Magnificus, Prof. mr. G.P.M.F. Mols, volgens het besluit van het College van Decanen, in het openbaar te verdedigen op vrijdag 4 februari 2011 om 12.00 uur

door

Florence Hubertine Johanna van Tienen

Geboren te Sittard op 21 augustus 1980 


\section{Promotor}

Prof. dr. H.J.M. Smeets

\section{Copromotores}

Dr. I.F.M. de Coo

Dr. C.J.H. van der Kallen

\section{Beoordelingscommissie}

Prof. dr. A.C. Nieuwenhuijzen Kruseman (voorzitter)

Prof. dr. E.E. Blaak

Prof. dr. J.F. Glatz

Prof. dr. J.A. Maassen (AMC, Amsterdam)

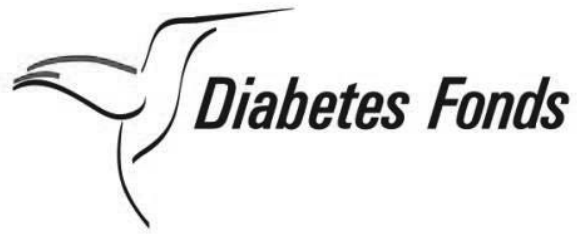

The studies described in this thesis were supported by a grant of the Dutch Diabetes Foundation (DFN 2004.00.040).

Printing of this thesis was financially supported by the Dutch Diabetes Research Foundation, which is gratefully acknowledged by the author. 
Experience is not the things that happened to you, but what you did with it

- A. Huxley - 



\section{Table of Contents}

\section{Chapter 1}

General introduction

\section{Chapter 2}

Skeletal muscle mitochondrial dysfunction is secondary to Type 2

Diabetes and can be improved by prolonged exercise training

\section{Chapter 3}

Gene expression profiling reveals differences in pathological processes in skeletal muscle of affected carriers of the m.3243A>G (MELAS) mutation

\section{Chapter 4}

Preadipocytes of Type 2 Diabetes patients display an intrinsic genetic expression profile of decreased differentiation capacity

\section{Chapter 5}

Wnt5b stimulates adipogenesis at the start of differentiation by inhibiting the canonical Wnt signalling pathway and activation of PPARY

\section{Chapter 6}

Prolonged Nrf1 overexpression triggers inflammation and insulin resistance in 3T3-L1 adipocytes

\section{Chapter 7}

General discussion 



\section{Chapter 1}

General Introduction 


\section{Diabetes Mellitus}

Diabetes Mellitus (DM) is a group of metabolic disorders of multiple aetiologies, resulting in chronically high blood glucose levels, which is also called hyperglycaemia. Depending on the cause, in which generally a combination of hereditary factors and environmental conditions are involved, hyperglycaemia can be the result of defective insulin secretion, diminished tissue response to the actions of insulin, or both. Hyperglycaemia is responsible for the acute signs of diabetes: excessive urine production, compensatory thirst and increased fluid intake, blurred vision, unexplained weight loss, lethargy, and changes in energy metabolism. Long-term complications of diabetes include retinopathy, nephropathy, neuropathy and cardiovascular dysfunction. Treatment generally involves lifestyle intervention as well as pharmacological treatment using insulin secretagogues, insulin sensitizers and/or exogenous insulin. The exact treatment strategy will depend on the cause and stage of the disease.

\section{Classification}

Based on the different causes known of DM, the expert committee of the American Diabetes Association (ADA) and the World Health Organization (WHO) proposed in 1997 a classification system of four categories:

- Type 1 Diabetes Mellitus (T1DM), also called Insulin Dependent Diabetes Mellitus (IDDM) or juvenile-onset diabetes. T1DM accounts for $5-10 \%$ of the diabetes patients and results from a T-cell mediated autoimmune response to pancreatic $\beta$-cells, leading to gradual destruction of the $\beta$-cells and failure to produce insulin [2]. The rate of $\beta$-cell destruction is quite variable, but the diagnosis T1DM is generally identified during childhood by manifestation of ketoacidosis. Since there is no cure, the therapy consists of exogenous insulin administration.

- Gestational diabetes. During pregnancy, a constant flow of glucose and other nutrients towards the foetus is facilitated by increased maternal insulin secretion and a physiological level of insulin resistance. A super-physiological level of insulin resistance 
and/or failure of $\beta$-cells to increase their activity results in gestational diabetes [4]. As a consequence, women with gestational diabetes have macrosomic babies, $60 \%$ of whom are born with caesarean sections. Gestational diabetes affects about $4 \%$ of all pregnancies and increases the risk of developing type 2 diabetes mellitus within 5 to 16 years after the index pregnancy with $17-63 \%$ [2].

- Other specific types of diabetes. This group comprises a number of monogenetic forms of diabetes. Genetic defects in both nuclear genes and mitochondrial (mt) DNA have been reported to affect $\beta$-cell function or insulin action, e.g. genetic defects in HNF-1a, HNF-4a, or mtDNA tRNAleu. Diseases or infections of the pancreas, endocrinopathies resulting in altered hormone secretion, drugs that interfere with insulin secretion or action, or other genetic syndromes associated with diabetes are also included in this class.

- Type 2 Diabetes Mellitus (T2DM). T2DM is the most common form of diabetes as it account for $90-95 \%$ of the diabetes patients. T2DM is the focus of this thesis and will be explained in more detail in the following paragraphs.

\section{Type 2 Diabetes Mellitus (T2DM)}

Insulin produced by the pancreatic $\beta$-cells is required for uptake of glucose from the bloodstream into peripheral tissue to generate energy (figure 1). This metabolic process is affected in T2DM. T2DM is characterized by high blood glucose levels due to reduced glucose uptake in peripheral tissues in response to insulin signaling, called insulin resistance, in combination with disinhibited hepatic glucose output and a reduced capacity of pancreatic $\beta$-cells to produce insulin. In the insulin resistant state, which precedes T2DM, impaired glucose tolerance in itself can be compensated by hyperinsulinaemia in order to maintain euglycaemia [8]. In this state, pancreatic islet $\beta$-cells produce an excessive amount of insulin. When $\beta$-cell are unable to compensate for the reduced insulin efficiency, and fasting blood glucose levels rise $\geq 7.0 \mathrm{mmol} / \mathrm{L}$, then T2DM is diagnosed [2]. The primary risk factor for development of T2DM is obesity, but physical inactivity, age, history of 


\section{Chapter 1}

cardiac disease, and a family history of T2DM contribute to the risk as well.

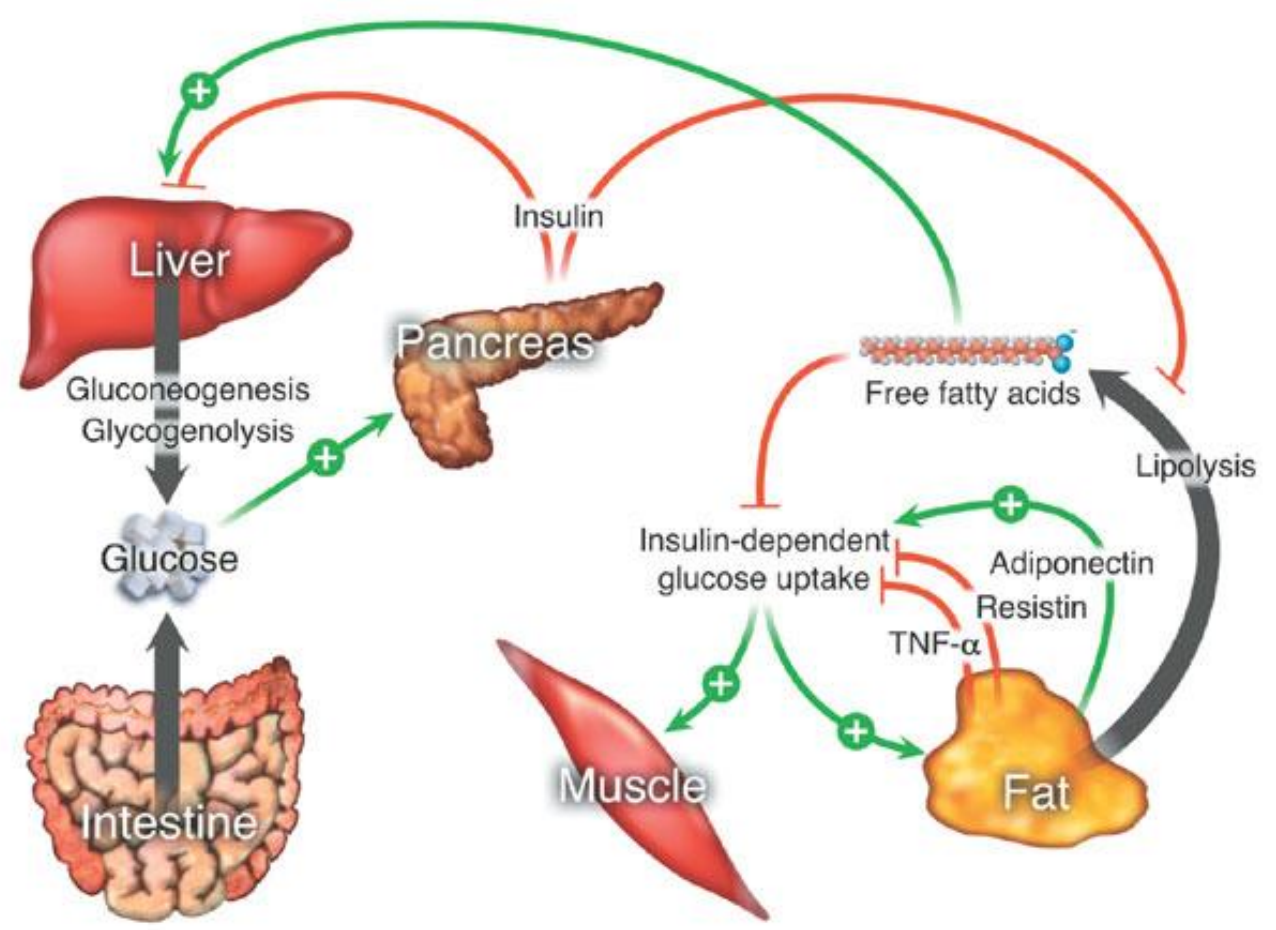

Fig. 1. Tissue cross-talk in glucose and lipid homeostasis in normal and diabetic subjects Normally, glucose derived from food or liver stimulates pancreatic insulin secretion. Insulin subsequently stimulates glucose uptake in muscle and fat tissue, while it inhibits gluconeogenesis and glycogenolysis in the liver and lipolysis and adiponectin secretion in the fat tissue. Obese and T2DM subjects generally show increased circulating plasma free fatty acids concentrations, which contribute to insulin resistance by inhibiting insulindependent glucose uptake. In addition, altered adipokine signaling, e.g. decreased adiponectin, and increased TNFa and Resistin, can inhibit insulin-stimulated glucose uptake [9].

The high blood glucose levels in T2DM can cause short-term complications like seizures and diabetic coma, and long-term consequences due to organ damage, e.g. heart and kidney disease, eye and foot complications, Alzheimer disease and increased stroke risk, eventually leading to a reduced life-span. Preventing the increase in blood glucose levels can dramatically reduce the risk of these complications. For many newly diagnosed T2DM patients, a healthy and balanced diet combined with regular physical activity is sufficient to maintain near normal glucose levels. If life-style changes are insufficient to control T2DM, medication is used that increase insulin sensitivity, 
reduce hepatic glucose output, and stimulate insulin production. When these drugs fail to lower the blood glucose level, exogenous insulin administration is required.

The incidence of T2DM increases dramatically and it is becoming a major health problem. In 2000, 171 million people (age $>20 \mathrm{yrs}$ ) were diagnosed with diabetes world-wide and the number of diabetes patients is expected to double within 30 years [10]. The three countries with the highest prevalence of diabetes in the world were respectively India, China and United States. The most recent data from 2007 is from the United States, where 23.6 million people $(7,8 \%)$ were diagnosed with diabetes, from which $90-95 \%$ is T2DM [11]. In the Netherlands, data from general practitioner registries showed that in 2003 over 600.000 people were diagnosed with diabetes [12] and the diabetes prevalence increased from $3 \%$ in $2002 / 2003$ to $4 \%$ in $2007 / 2008$ [13].

\section{Energy metabolism}

Energy metabolism is the complex of physical and chemical processes within a living cell that yield ATP, the cellular source of energy, by breaking down or synthesizing specific cellular substances. As T2DM results from disturbed energy metabolism, it is essential to understand the normal process. Energy metabolism involves multiple organs, e.g. (skeletal) muscle, adipose tissue, pancreas and liver, which break-down or store metabolic compounds in normal energy metabolism. In the following paragraphs the energy metabolism and the role of the main organs or tissues involved will be discussed.

\section{Generation of cellular energy}

Carbohydrates, proteins and lipids are the main sources of energy. Oxidation of these metabolic fuels is coupled to biosynthesis of ATP, which is required for virtually all biological processes in mammals (figure 2). The majority of ATP is generated by oxidative phosphorylation in the mitochondria. Upon ingestion, carbohydrates, proteins and lipids are processed in different pathways into acetyl coenzyme A (acetyl-CoA), which is oxidized through the citric acid cycle and electron transport chain. Carbohydrate metabolism starts with anaerobic glycolysis in the 


\section{Chapter 1}

cytosol, which releases energy from glucose into two molecules of pyruvate, which are translocated to the mitochondria. Proteins are digested into amino acids, which can be resynthesized in new proteins or other compounds, but about $10 \%$ is used for energy production. After deamination, removing the nitrogen group, the remaining carbon skeleton can be converted into acetyl CoA directly, or into pyruvate, or a citric acid cycle intermediate. Finally, fatty acids are derived from ingested lipids or generated from carbohydrates or amino acids. They react with ATP to give a fatty acyl adenylate plus inorganic pyrophosphate. This reactive acyl adenylate then reacts with free coenzyme A ( CoA) to give a fatty acyl-CoA ester that can be transported to and metabolized in the mitochondria.

In the mitochondrial matrix, pyruvate and fatty acyl CoA generate via the Krebs cycle and $\beta$-oxidation ATP, but also $\mathrm{NADH}$ and $\mathrm{FADH}_{2}$, which are molecules that can enter the oxidative phosphorylation system (OXPHOS) (figure 2). Oxidative phosphorylation starts with a series of four oxidation/reduction reactions during which an electrochemical gradient is created by pumping protons from the mitochondrial matrix into the mitochondrial inter-membrane space. This electron built-up is energetically coupled to an exergonic reaction by the ATPase complex that drives the condensation of ADP and inorganic phosphate into ATP. The formed ATP is subsequently transported out of the mitochondrial matrix into the cytosol by the adenine-nucleotide transporter (ANT1) [14].

Because the majority of ATP is generated in the mitochondria, they are called the energy factories of a cell. The number of mitochondria present in a cell depends on the energy requirement of the tissue, e.g. skeletal muscle requires more ATP production to function than adipocytes. Mitochondria are also the only organelles in cells apart from the nucleus, which contain their own maternally transmitted DNA. The mtDNA is a double-stranded circular molecule of 16,569 bp, which comprises 22 tRNA genes, 2 rRNA genes and 13 protein coding genes. Replication of the mtDNA starts from a non-coding region, called the displacement loop (D-loop). The proteins encoded by the mtDNA are subunits of the OXPHOS complexes I, III, IV and V. The other subunits of these complexes and the entire complex II are encoded by the nuclear DNA. Mutations, i.e. point mutations, deletions or duplications in the mtDNA, 
can lead to dysfunctional OXPHOS and clinical symptoms especially in energy consuming tissues like brain and skeletal muscle. Since each mitochondrion contains multiple copies of mtDNA and both wild-type and mutated mtDNA can be present in a cell, mutation percentages can vary between $0 \%$ and $100 \%$. Due to a high replication rate, a limited repair mechanism and high availability of ROS, the mtDNA mutation rate is about 10-20 times higher than the nuclear DNA [15, 16]. Therefore, the clinical effect of the mutation depends on both the location of the mutation in the mtDNA and the quantity. During the process of oxidative phosphorylation, reactive oxygen species (ROS) are formed. ROS are ions or very small molecules that include oxygen ions (superoxide), free radicals, and peroxides (hydrogen peroxide), both inorganic and organic. They are highly reactive due to unpaired electrons, and they have important roles in cell signaling [17]. However, as a result of environmental stress or dysfunctional OXPHOS, ROS levels can increase dramatically, and damage RNA, DNA and proteins. This ROS accumulation is also known as oxidative stress [18].

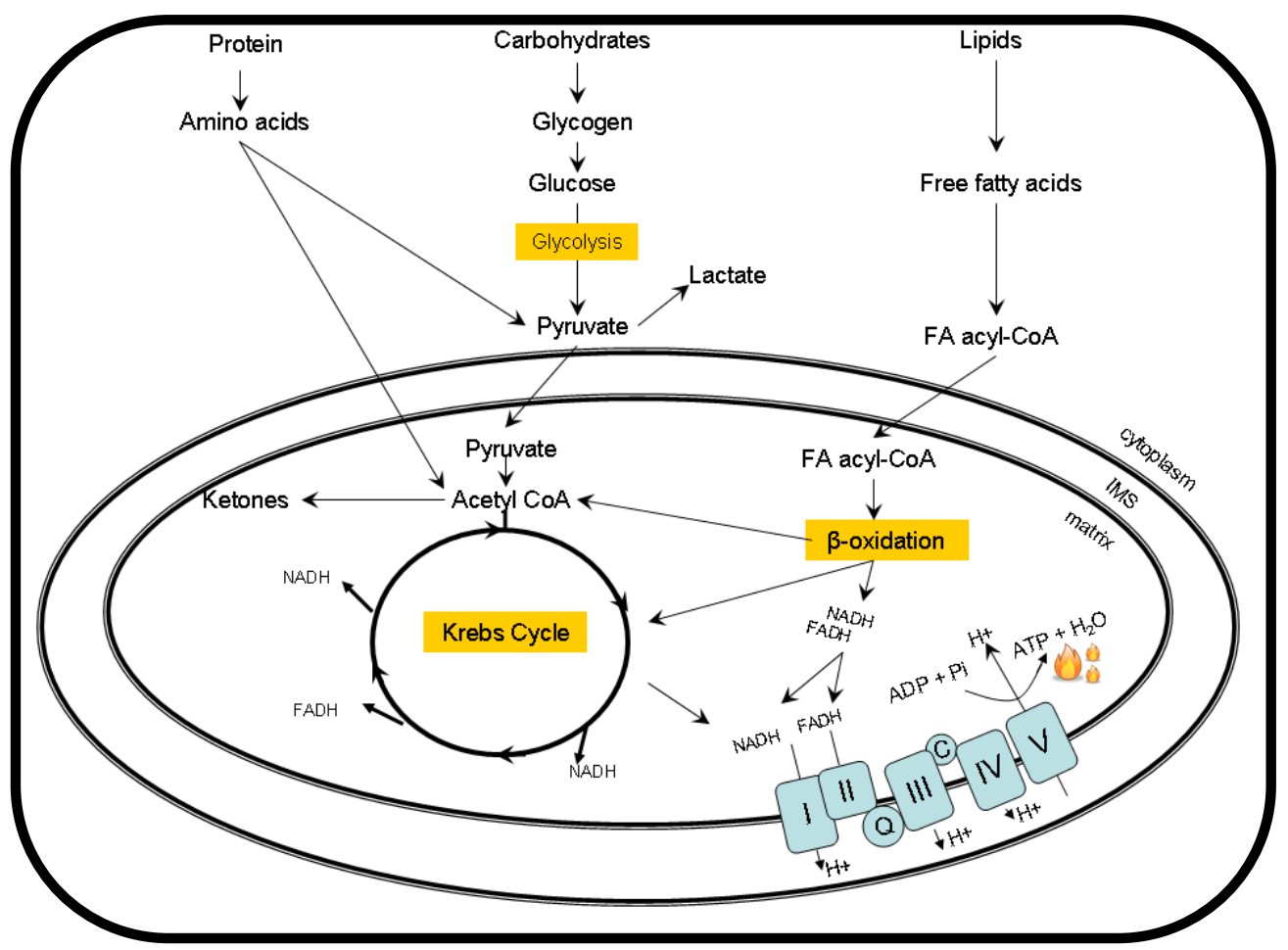

Fig. 2. Energy generation from proteins, fat and carbohydrates [19]. 


\section{Chapter 1}

\section{Adipose tissue}

Adipose tissue is composed of various subcutaneous and visceral depots throughout the body. It consists of mature energy storing adipocytes, adipocyte precursor cells called preadipocytes, and stromal vascular cells (SVCs). In the past, adipose tissue was predominantly regarded as a passive reservoir for storage of lipids in the form of triglycerides. Adipose tissue is an insulin sensitive organ, insulin can mediate glucose uptake that can also be converted to fatty acids, and prevents breakdown of triglycerides by inactivation of hormone-sensitive lipase (HSL). In contrast, glucagon activates HSL, causing the breakdown of the stored triglycerides into fatty acids, which are exported into the blood. Fatty acids are taken up by muscle and cardiac tissue as a fuel source, and glycerol is taken up by the liver for gluconeogenesis. Nowadays, adipose tissue is regarded as an endocrine organ, as it secretes locallyand systemic acting proteins, termed adipokines, e.g. IL-6, resistin, adiponectin and leptin. These adipokines act in tissues such as liver, skeletal muscle and brain to modulate insulin action. Other adipokines, e.g. TNF- $a$ and growth factors, act in an autocrine/paracrine fashion and play a role in adipose tissue proliferation and differentiation [20]. The key role of adipose tissue in energy homeostasis and its influence on insulin sensitivity is emphasized by the fact that lack of sufficient adipose tissue amounts, as seen with lipodystrophy, is associated with insulin resistance [21].

Before differentiation of preadipocytes into adipocytes, growth arrest of preadipocytes is required, which is followed by one round of DNA replication and cell doubling, termed clonal expansion of committed cells. This process is accompanied by drastic changes in gene expression, which is under tight control of hormones, e.g. insulin and glucocorticoids, nutrients, cytokines and signaling molecules, changing expression of a number of transcription factors that subsequently influence the conversion into adipocytes. Key transcription factors in adipogenesis are the families of CCAAT-enhancer binding proteins $(C / E B P-a,-\beta$, and $-\delta)$, peroxisome proliferator-activated receptors $($ PPAR $-\gamma,-\beta,-\delta)$, and sterol regulatory element binding transcription factor 1c (SREBP-1c), which is a member of the basic helix-loop-helix (bHLH) transcription factor family [22]. Morphologically, the fibroblastlike preadipocyte converts into round lipid-filled adipocytes. This process starts within the first 12 hours of differentiation and involves dramatic 
changes in cell morphology, extracellular matrix and cytoskeleton [23]. Late events in adipogenesis involve increased lipogenic capacity and acquired insulin sensitivity [24]. In addition to adipogenesis promoting factors, other factors inhibit adipogenesis, and the adipogenic capacity depends on the balance between these factors. Inflammatory cytokines, e.g. interleukins (IL-1, IL-6, IL-11), tumour necrosis factor a (TNF-a), and oncostatin $M$ inhibit adipogenesis by reducing PPARY and $C / E B P a$ expression. Transforming growth factor $\beta$ (TGF- $\beta$ ), platelet derived growth factor (PDGF) and epidermal growth factor (EGF) on the other hand, inhibit differentiation by stimulating proliferation. Other factors, like members of the Wnt family of secreted glycoproteins can inhibit adipogenesis, e.g. by Wnt1 and Wnt10b overexpression [25], but also stimulate adipogenesis by Wnt5a and Wnt5b overexpression $[26,27]$.

\section{Skeletal muscle}

Skeletal muscle tissue is attached to bones, and in contrast to cardiacand smooth muscle, it can contract or relax by conscious control. Both glucose and FFA are the primary fuels for skeletal muscle metabolism. About $85 \%$ of glucose disposal under conditions of glucose infusion can be accounted for by insulin-stimulated glucose uptake into muscle [28]. Glucose uptake in cells is mediated by members of the glucose transporter (GLUT) family, and the main glucose transporter in cardiacand skeletal muscle and adipose tissue is GLUT4, which mediates insulin induced glucose uptake [29].

Insulin facilitates glucose uptake by skeletal muscle when blood glucose availability rises. The insulin signaling pathway starts with binding insulin to the insulin receptor (IR) on the cell membrane, leading to IR autophosphorylation with catalyzing phosphorylation of intracellular substrates, including insulin receptor substrate (IRS) proteins, adapter protein APS, and Cbl. These substrates then recruit distinct subsets of signaling molecules and either the classical insulin signaling cascade or the phosphoinositol 3-kinase (PI-3K) independent cascade is induced. The classical insulin signaling cascade starts with IRS-dependent activation of PI-3K, generating phophoinositol 3-phosphatase (PIP3) that subsequently activates phophoinoside-dependent kinase 1 (PDK1), protein kinase $B(P K B)$ and atypical protein kinase family members $(\mathrm{PKC} \lambda / \zeta)$, resulting in translocation of the muscle glucose transporter (GLUT4) from the intracellular storage sites to the plasma membrane, 


\section{Chapter 1}

augmenting glucose transport into the cell [29] (figure 3). The PI-3K independent signaling cascade involves phosphorylation of $\mathrm{Cbl}$ and recruitment of the CAP-CrkII-C3G complex, which translocates to the lipid raft microdomain where it activates GTP-binding protein TC10 and directly translocates GLUT4 to the plasma membrane [30]. Besides signaling glucose uptake, insulin has additional functions in skeletal muscle. It stimulates amino acid uptake and protein synthesis, inhibits protein degradation, elicits activation of the mitogen activated protein kinase (MAPK) cascade leading to mitogenic responses and promotes storage of carbohydrates in the form of glycogen, lipids and proteins [31].

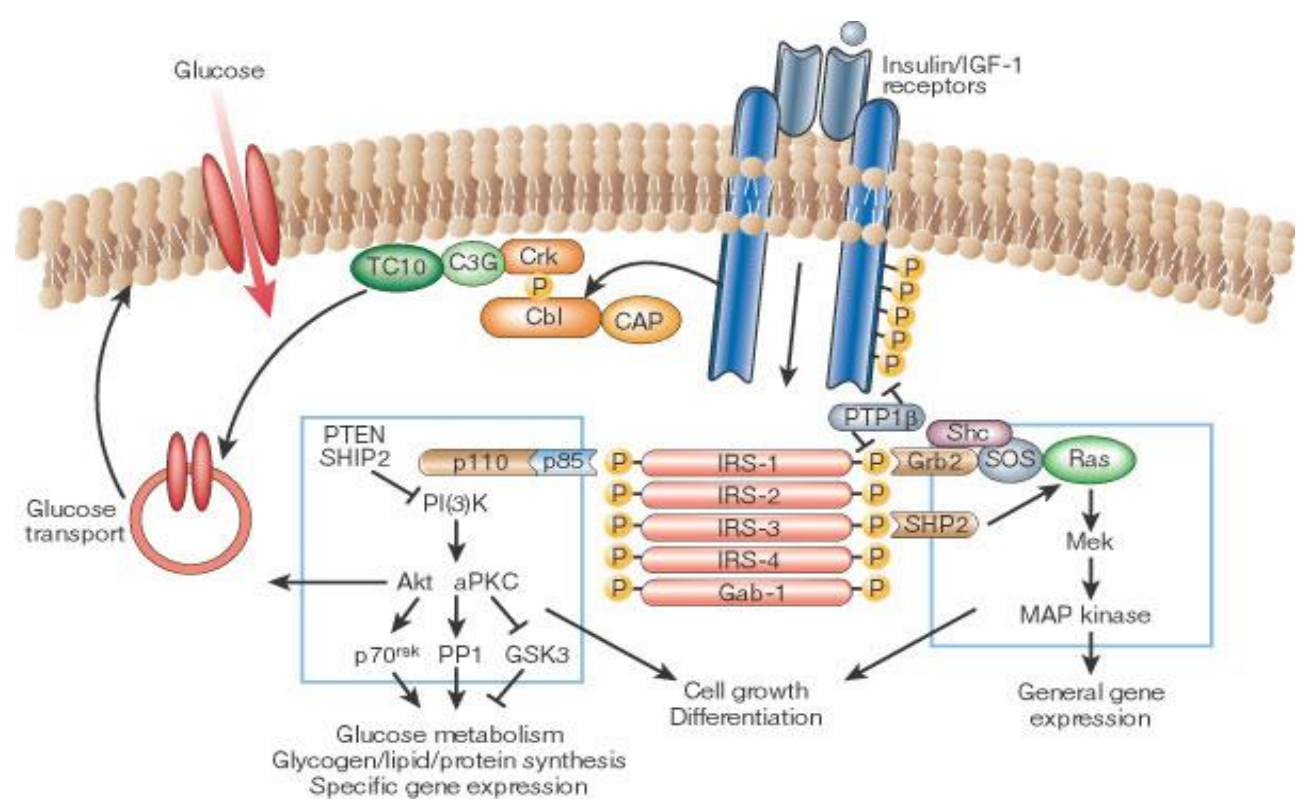

Fig. 3. Insulin mediated GLUT4 translocation for glucose uptake [31].

Skeletal muscle is made up of bundles of individual muscle fibers, called myocytes. These fibres are not identical in structure and function and their contraction rates depend on their ability to generate ATP. Based on various structural and functional characteristics, skeletal muscle fibres are classified into three types: type I fibres, type IIa fibres and type IIb fibres. Type I fibres are so called slow contracting fibres with a small fibre diameter, high mitochondrial and myoglobin content, and high triglyceride storage. They contain less of the enzymes involved in glycolysis, but contain more of the enzymes involved in the oxidative 
pathways. Functionally, type I fibres are used for aerobic activities requiring low-level force production, e.g. walking and maintaining posture. In contrast, type II fibres are called fast contracting fibres because of the quicker rate of calcium release and higher activity of myosin-ATPase that breaks down ATP compared with type I fibres [32, 33]. Type II fibres are further divided into IIa and IIb. Type IIa fibres represent a transition between the two extremes of the type I and type IIb fibres. Structurally, they have a large fibre diameter, a high mitochondrial density, a medium capillary density, and medium myoglobin content. They are high in creatine phosphate and glycogen and medium in triglyceride stores. They have both a high glycolytic and oxidative enzyme activity. Functionally, they are used for prolonged anaerobic activities with a relatively high force output, type IIb fibers on the other hand are used for short anaerobic, high force production activities, such as sprinting. These fibres are also capable of producing more power than type I fibres. Type IIb fiber has a similar fiber diameter as type IIa, but with low mitochondrial and capillary density and myoglobin content. They also are high in creatine phosphate and glycogen, but low in triglycerides. They contain more glycolytic enzymes and less oxidative enzymes. In addition to fibre type differences, the location of mitochondria determines their function, as subsarcolemmal mitochondria provide ATP for energy consuming processes like ion and metabolite transport and cell signaling, while intermyofibrillar mitochondria are thought to be more responsible for generating energy for muscle contraction [34].

\section{Liver}

The liver is an important metabolic organ with a number of functions, including detoxification, haemoglobin breakdown, production of coagulation factors and albumin, storage of vitamins and glucose, immunological effects, breakdown of insulin and other hormones, carbohydrate and lipid metabolism. With regard to carbohydrate metabolism, the liver plays a role in the synthesis of glucose from amino acids, lactate or glycerol (gluconeogenesis) and from glycogen (glycogenolysis). In addition to synthesizing glucose, glucose can also be converted into glycogen by the process of glycogenesis. The liver plays an important role in maintaining physiologically normal blood glucose levels with insulin stimulating the uptake and glycogen synthesis of excessive glucose. In contrast, when blood glucose levels are too low, 


\section{Chapter 1}

more glucagon and less insulin is secreted from the pancreas and the liver stops glycogenesis and starts gluconeogenesis to increase blood glucose levels. In lipid metabolism, the liver is involved in cholesterol and phospholipids synthesis and is extremely active in oxidizing triglycerides to produce energy. A bulk of the lipoproteins are synthesized in the liver and it is the major site for converting excess carbohydrates and proteins into fatty acids and triglyceride (lipogenesis), which are then exported and stored in adipose tissue.

\section{Pancreas}

The pancreas is a gland of the vertebrate endocrine system, which produces important hormones, including insulin, glucagon, and somatostatin, which are involved in glucose metabolism. These three hormones are, respectively, produced by the $\beta$-, a- and $\delta$-cells of the Islets of Langerhans.

- Insulin is responsible for the majority of glucose uptake from the blood by liver, muscle, and adipose tissue, storing the glucose as glycogen in the liver and muscle, and inhibiting the use of fat as an energy source (see fig. 1).

Insulin secretion from the pancreatic $\beta$-cells requires a rise in ATP:ADP ratio from glycolysis leading to closure of potassium channels. Insulin exocytosis is subsequently triggered by activation of voltage-gated calcium channels [35]. Glucose is the predominant stimulator, but insulin release can also be triggered by fatty acids, amino acids and hormones [36-38]. The insulin level is a central metabolic control mechanism. When insulin is low or absent, glucose is not taken up anymore by most body cells and the body begins to use fat as an energy source.

- Glucagon is involved in carbohydrate metabolism with an opposite action compared with insulin. Glucagon is released when the glucose level in the blood is low (hypoglycemia), causing the liver to convert stored glycogen into glucose and release it into the bloodstream. However, glucagon also stimulates the release of insulin to stimulate the uptake of newly available glucose in the bloodstream by insulin-dependent tissues.

- Somatostatin is referred to as an inhibitory hormone which is secreted in the stomach, intestine and by pancreas and among other things inhibits insulin and glucagon release from the pancreas. 
The islets of Langerhans only make up $1-2 \%$ of the mass of the pancreas. The major part of the pancreas are lobules that produce and secrete pancreatic juice containing digestive enzymes that pass to the small intestine, which help the further breakdown of the carbohydrates, protein, and fat.

\section{Pathophysiology of Type 2 Diabetes Mellitus (T2DM)}

Development of T2DM is a gradual process, which is preceded by insulin resistance of tissues and organs involved in glucose homeostasis, like skeletal muscle, adipose tissue and liver. The insulin resistant state requires increased insulin production by the pancreatic $\beta$-cells to facilitate glucose uptake. The inability of $\beta$-cells to do so results in development of T2DM. The following sections will give an overview of the changes in these organs during the development of insulin resistance and T2DM.

\section{Adipose tissue dysfunction}

Insulin resistance is generally defined with a glucocentric view, but can also be regarded from a lipocentric view, since excess fatty acids, which are generally a consequence of obesity, are a major factor predisposing to the insulin resistance and hyperinsulineamia that precede hyperglycaemia [39, 40]. An inverse relation exists between adipose tissue mass and insulin sensitivity [41, 42], indicating that efficient adipose tissue functioning and storage of fatty acids is important for normal glucose metabolism. Adipose tissue is considered an important endocrine organ, which secretes a number of adipokines and is involved in $5-10 \%$ of the insulin-mediated glucose disposal $[43,44]$. In lean individuals, adipose tissue stores excessive energy in the form of triglycerides, and there is a dynamic balance between fatty acid release and use in peripheral tissues for energy production. Obesity is characterized by excess energy intake that leads to high availability of fatty acids. Although the adipocytes are capable of storing excess lipids by enlargement of adipocytes, prolonged excess energy intake results in hypertrophic adipocytes [45]. Hypertrophic adipocytes create a stress condition and have an altered adipokine secretion pattern. Adipokines, which modulate the inflammatory response are increased, e.g. TNFa and MCP-1, whereas adiponectin, which is involved in fatty acid oxidation is 


\section{Chapter 1}

reduced [46-49]). TNFa and MCP-1 function as chemoattractants, enhancing macrophage infiltration into adipose tissue of obese people [50]. As a result, the amount of macrophages in adipose tissue of obese people is $\sim 50 \%$ of total cell number, compared to $5-10 \%$ in lean individuals [51]. TNFa secreted from both adipocytes and macrophages affects adipocyte function profoundly by induction of NF-kB and JNK-AP1 pro-inflammatory signaling pathways. Downstream effects depend on the cellular context, but include apoptosis, ER stress, mitochondrial dysfunction, reduced differentiation capacity, stimulation of inflammation and insulin resistance [52]. Taken together, hypertrophic inflamed adipocytes have a decreased uptake, utilization and storage of lipids, and at the same time enhanced transport of free fatty acids out of the adipocytes by lipolysis. This leads to increased lipid availability in the circulation and increased uptake of fatty acids by peripheral tissues, e.g. skeletal muscle, liver and pancreas. In non-adipose tissues, accumulation of free fatty acids in the cytoplasm, which is also called lipotoxicity, can impair normal cell function causing cell dysfunction and possibly cell death [53]. The pro-infammatory adipokine TNFa plays an important role in these processes.

As shown in figure 4, increased TNFa affects multiple processes in adipocytes. Besides affecting lipid handling capacity, TNFa can induce physiological insulin resistance in adipocytes by interfering with the insulin signaling pathway directly. It inhibits proteins required for insulin-stimulated glucose uptake, such as Ser/Thr phophorylation of the insulin receptor substrate (IRS) proteins and the insulin receptor itself [54]. At the transcriptional level, TNFa inhibits GLUT4 expression that is required for glucose uptake [55]. A combination of increased inflammation together with abolished insulin signaling reduces adipocyte function and regeneration capacity even further. In addition, TNFamediated inflammatory response can down-regulate expression of key transcriptional regulators of adipogenesis, like $P P A R Y$ and $C / E B P a$, via NF-kB signaling [56], and degrade PPARY mRNA post-transcriptionally via MAP4K4 signaling [57]. Additionally, insulin resistance of adipose tissue also prevents PPARY and C/EBPa expression, and GATA2 and FOXO1 phosphorylation, inhibiting adipogenesis further [58, 59]. Free fatty acid availability in the circulation is enhanced due to TNFamediated inhibition of PPARY-mediated uptake, synthesis, hydrolysis and deposition of triglycerides in the lipid droplet, which is mediated by 
lipoprotein lipase (LPL), phosphoenolpyruvate carboxykinase (PEPCK), and fatty acid synthase (FAS). Furthermore, TNFa inhibits lipid-droplet enlargement by CIDE-domain containing proteins FSP27 and CIDEA [6063], and enhances lipolysis of free fatty acids through cAMP and protein kinase A (PKA) mediated phosphorylation of $\mathrm{HSL}[64,65]$. All these processes enhance free fatty acid availability in the circulation, predisposing to insulin resistance and hyperinsulineamia.

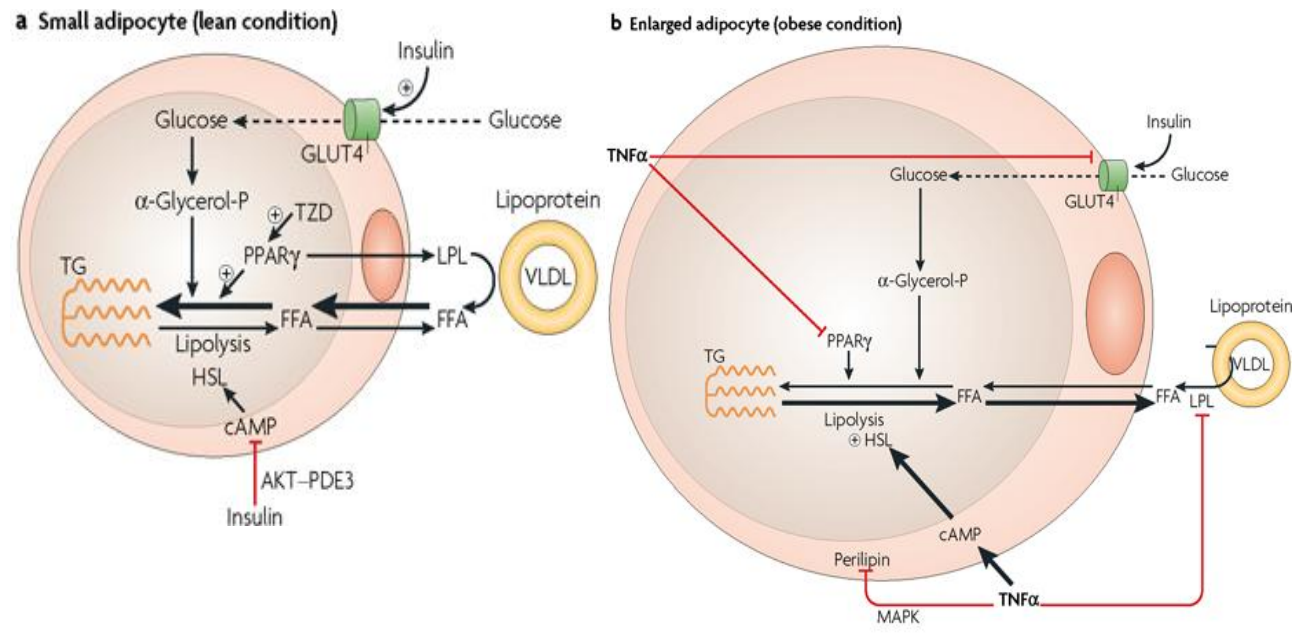

Fig. 4. Metabolism in small and enlarged adipocytes [45].

a) In small adipocytes (lean condition), insulin promotes free fatty acid (FFA) esterification into triglycerides (TG) through stimulation of glucose transporter type-4 (GLUT4)-mediated glucose uptake. Glucose can be converted to a-glycerol phosphate, the main source of the glycerol backbone of TG. Peroxisome proliferator-activated receptor-y (PPARY) activates lipoprotein lipase $(L P L)$ expression and the TG biosynthetic pathway. Secreted LPL hydrolyses TG from circulating very low-density lipoprotein (VLDL), releasing FFAs to be re-esterified. Several thiazolidinediones (TZDs) can activate PPAR. Insulin signaling also downregulates TG lipolysis through hormone-sensitive lipase (HSL). Insulin stimulation of the phosphatidylinositol 3-kinase (PI3K)-AKT/protein kinase B (PKB) pathway leads to activation of the enzyme phosphodiesterase-3 (PDE3). This enzyme catalyses the breakdown of cyclic AMP (CAMP) which in turn reduces activation of HSL.

b) In enlarged adipocytes from inflamed adipose tissue, high levels of tumour necrosis factor a (TNFa) result in decreased fatty acid esterification and enhanced lipolysis. GLUT4, LPL and PPAR protein levels are attenuated by TNFa, resulting in decreased glucose transport and fatty acid esterification. TNFa also has a stimulatory effect on lipolysis by increasing the levels of CAMP and activation (plus signal) of HSL, combined with the downregulation of perilipin through activation of the mitogen-activated protein kinase (MAPK) pathway. 


\section{Chapter 1}

Skeletal muscle insulin resistance

Free fatty acids from the circulation enter skeletal muscle through the fatty acid transporter protein (FATP) and increase the acyl-CoA pool. In skeletal muscle, the lipids are either transported to the mitochondria for $\beta$-oxidation or they are stored as intramyocellular lipids (IMCLs) like triglycerides, diacylglycerol (DAG) and ceramides [66, 67]. A negative relation between IMCL content and insulin sensitivity has been frequently reported, even in young lean first-degree relatives of T2DM patients [68-72]. This indicates that free fatty acids in skeletal muscle are key players in the development of T2DM. Paradoxically, trained insulin sensitive athletes also have increased IMCL content and physical activity is associated with increased insulin sensitivity, but also increased IMCL. Training rather affects the distribution of intramyocellular lipids than the quantity of $I M C L$, since training is associated with a shift from diacylglycerol and ceramide towards more intramyocellular triglycerides [73].

Fatty acid induced insulin resistance was already demonstrated more than 40 years ago by Randle et al., who hypothesized that this was the result of substrate competition between fatty acids and glucose, as increased fatty acid oxidation would enhance the acyl-CoA level, which would in turn inhibit key enzymes of glycolysis [74-76]. However, current data indicate that intramyocellular fatty acids do not compete with glucose, but inhibit the insulin signaling pathway directly at the initial signaling steps by inhibition of tyrosine phosphorylation and by stimulating serine phosphorylation of insulin receptor and IRS proteins [77]. In addition to high availability of fatty acids, altered adipokine signaling from insulin-resistant adipose tissue affects muscle metabolism. For example adiponectin and leptin, which are involved in skeletal muscle glucose and fatty acid oxidation, respectively, are negatively correlated with insulin resistance [78-82]. In contrast, the pro-inflammatory adipokines TNFa and IL- 6 that interfere with insulin signaling are positively correlated with insulin resistance [83-85]. Since skeletal muscle is responsible for about $80 \%$ of the insulin stimulated glucose disposal, it is regarded as one of the most insulin-sensitive organs [86]. Disruption of skeletal muscle insulin signaling is therefore one of the earliest hallmarks of insulin resistance, even 10-20 years before onset T2DM, and a major contributor to the development of T2DM $[87,88]$. Skeletal muscle insulin resistance is characterized by 
reduced glucose transport and phosphorylation [89], reduced rate of glycogen synthesis [90], abnormal fatty acid metabolism, and reduced lipid oxidation during fasting and insulin-stimulated conditions [91, 92].

In T2DM patients a shift from the oxidative fiber-type 1 towards more glycolytic fiber-type 2 has been observed. This was associated with a reduction in mitochondrial content and in ATP production [93, 94]. ATP production capacity was also reduced in young lean offspring of T2DM subjects who had a reduced insulin-mediated glucose uptake. Based on these data, it was hypothesized that mitochondrial dysfunction resulted in a decreased fatty acid oxidation and enhanced IMCL content, contributing to insulin resistance. The exact role of mitochondrial quantity and functioning in T2DM remains to be identified, as a growing number of conflicting data on the topic appears. An overview of studies that have explored changes in mitochondrial quantity, quality and functioning in skeletal muscle of T2DM patients will be discussed in the next paragraph.

\section{Hepatic insulin resistance}

In addition to skeletal muscle, free fatty acids from the circulation are also taken up by the liver, converted into triglycerides and stored as hepatocellular lipids or secreted as very-low-density-lipoproteins (VLDL). Accumulation of hepatocellular lipid ( $\mathrm{HCL}$ ) is positively correlated with insulin resistance $[95,96]$, and more than $70 \%$ of T2DM subjects have a fatty liver [97]. Hepatic insulin resistance is characterized by high lipid availability, hyperglycemia and hyperinsulinemia. In the liver so-called selective insulin resistance is observed, which is characterized by impaired insulin-mediated inhibition of gluconeogenesis and impaired glycogen synthesis, via IRS-2 and Fork-head box 01 (FoxO1). In contrast, lipogenesis is stimulated by insulin induced $P P A R Y$ and SREBP1C expression [98, 99]. In insulin resistant hepatocytes, JNK signaling can be activated directly via increased TNFa, fatty acids or glucose, or indirectly as a result of OXPHOS dysfunction. Activated JNK signaling inactivates IRS-1 and insulin signaling, creating hepatic insulin resistance and elevation of the plasma glucose level [100-103].

The fatty liver of T2DM patients is a major risk for non-alcoholic steatohepatitis (NASH) and subsequent fibrosis [97]. Mitochondrial functioning plays a key role in disease progression. The high availability 


\section{Chapter 1}

of fatty acids initially increases mitochondrial $\beta$-oxidation, which also augments formation of reactive oxygen species (ROS) [104-106]. The high production of ROS in a lipid loaded environment can induce OXPHOS damage and reduce ATP production. Mitochondrial abnormalities have been observed in patients, who have progressed from compensated steatosis towards steatohepatatitis [107, 108], which implicates a key role for mitochondrial dysfunction in disease progression [99].

\section{Pancreatic $\beta$-cell failure}

The major difference between insulin resistance and full-blown diabetes is pancreatic $\beta$-cell failure. In the insulin resistant state, high glucose level in the circulation increases insulin secretion from the pancreatic $\beta$ cells to allow glucose uptake by peripheral tissues and results in a reduction of glucose in the circulation. This high demand for insulin production requires both expansion of the $\beta$-cell mass and enhanced $\beta$ cell functioning. Failure to expand and to produce sufficient insulin results in reduced uptake of glucose in peripheral tissues and high glucose in the circulation, hence development of T2DM. At diagnosis of T2DM, the $\beta$-cells generally operate at less than $25 \%$ of their functional capacity, of which $\sim 50 \%$ is contributed for by decreased $\beta$-cell mass due to apoptosis [109]. In addition to reduced $\beta$-cell mass, $\beta$-cells of T2DM patients show a decreased responsiveness to produce insulin. This reduced responsiveness is specific for T2DM and is not present in T1DM in which disease development is solely the result of severely reduced $\beta$ cell mass [110]. The current hypothesis for $\beta$-cell failure is that continuous excess of fatty acids leads to increased acyl-CoA, constitutive up-regulation of pyruvate cycling and increased basal insulin hypersecretion, while at the same time glucose induced pyruvate cycling and insulin secretion is suppressed. In contrast to other tissues, current data indicates that $\beta$-cell failure occurs as a consequence of increased fatty acid oxidation in $\beta$-cells, rather than of decreased fatty acid oxidation [111]. Chronic lipid exposure may also affect mitochondrial functioning, like changes in ATP:ADP ratio and membrane potential, proton leakage via UCP2 or increased oxidative stress, which might also contribute to decreased insulin production and $\beta$-cell death $[112,113]$. Insulin secretion is also influenced by growth factors and hormones, which are deregulated in T2DM, like decreased secretion of adiponectin and leptin resistance, which reduce insulin secretion and increase fatty 
acid depositioning in the pancreas [38, 114]. Additionally, insulin is accompanied by amyloid in the secretory granules [115], and increased insulin biosynthesis creates endoplasmatic reticulum stress and increased formation of toxic amyloid fibers. By a presently unknown mechanism, abnormal amyloid fibers are deposited in the $\beta$-cells of T2DM patients, and these amyloid fibers induce $\beta$-cell degradation and apoptotic cell death $[116,117]$. Due to reduced $\beta$-cell mass and functioning, the pancreatic $\beta$-cells fail to produce sufficient insulin for glucose uptake by peripheral tissues, the circulating glucose will be continuously elevated, which can cause organ and tissue damage.

\section{The role of mitochondria in T2DM pathophysiology}

A number of studies have demonstrated that mitochondrial dysfunction plays a key role in development of T2DM. Alterations like decreased mitochondrial size, decreased expression of genes involved in mitochondrial biogenesis and altered mitochondrial activity have been observed. However the picture is still far from clear. There are a number of conflicting reports on the role of mitochondria in the development of diabetes. In the next sections, different aspects of mitochondrial functioning and alterations in skeletal muscle and adipose tissue from insulin resistant and T2DM patients will be discussed.

\section{Mitochondrial quantity and morphology in T2DM}

The number of mitochondria differs between cell-types depending on their energy requirement. The number of intact mitochondria is commonly assessed by measuring Citrate Synthase (CS) activity, or by quantifying the mtDNA/nDNA ratio, which is also called the mtDNA copy number. Subcutaneous adipose tissue of obese insulin resistant subjects was shown to have a markedly lower mtDNA copy number than that of younger leaner subjects. Treatment of insulin resistant subjects with pioglitazone, which stimulates PPARY, for 12 weeks was shown to restore the mtDNA copy number and CS activity to the same level as the lean young control group [118]. Skeletal muscle mitochondrial quantity and quality has been more intensively studied than adipose tissue, and a number of groups have observed reduced mitochondrial content in skeletal muscle of T2DM patients when compared to lean controls with a 


\section{Chapter 1}

significantly lower BMI [5, 119, 120]. However, when compared with BMI-matched controls, only Kelley et al. observed decreased mitochondrial content, while other studies did not observe significant differences in mitochondrial content between insulin sensitive and T2DM subjects matched for BMI [5, 119-124]. On the other hand, the mtDNA copy number is found to be reduced in normoglycemic sedentary young males with a positive family history of T2DM compared with those without a family history of T2DM [125]. Additionally, physical activity is another factor involved, as exercise training correlates with increased mitochondrial content [126]. Current data indicates that reduced mitochondrial content in skeletal muscle is more a feature of obesity or an intrinsic metabolic defect rather than a risk factor for the development T2DM.

Apart from a reduced mitochondrial content, altered mitochondrial morphology has been observed in skeletal muscle of T2DM subjects. While subsarcolemmal mitochondria have not been specifically studied, morphologic analysis of intermyofibrillar mitochondria from lean, obese and obese T2DM subjects indicated a $\sim 35 \%$ reduced mitochondrial size in obese and T2DM subjects when compared with controls [5]. The intermyofibrillar mitochondria area was also shown to correlate positively with insulin sensitivity. A combined physical activity training and weight loss program increased the intermyofibrillar mitochondrial size and density, and also increased insulin sensitivity in obese insulin resistant subjects [127]. Similar results were also obtained with T2DM patients in another study, although no discrimination between intermyofibrillar and subsarcolemmal mitochondria was made [128]. In addition to the smaller mitochondria, also other aberrations were observed in intramyofibrillar mitochondria of T2DM subjects, like degenerated mitochondria and mitochondria with altered internal structure [5]. 
Oxidative stress and T2DM

Hyperglycemia has been shown to cause oxidative stress and is implicated in T2DM and associated renal, neurological and endothelial complications. Oxidative stress is thought to activate stress kinases, like JNK and NF-kB, which interfere with insulin signaling via serine phosphorylation of IRS1 [129]. The $\mathrm{H}_{2} \mathrm{O}_{2}$ emission rate reflects the balance between the superoxide formation rate in the OXPHOS system and the scavenging capacity in the mitochondrial matrix [130]. Studies with mice on high-fat high-sucrose diet, which developed diabetes, showed that the diabetic state, but not the insulin resistant stage, was associated with increased oxidative stress and altered mitochondrial quantity and functioning. Reducing oxidative stress by adding antioxidants improved mitochondrial integrity. In contrast, studies with obese and insulin resistant $\mathrm{KKA}^{\mathrm{V}}$ mice without hyperlipidemia, but with only mild hyperglycemia, did not experience local oxidative stress and mitochondrial dysfunction, indicating that most likely high circulating lipid levels are required to cause oxidative stress [131]. A similar observation was recently made by Anderson et al., who showed that a high fat diet directly caused increased $\mathrm{H}_{2} \mathrm{O}_{2}$ production in skeletal muscle of obese human and obese mice [132]. In contrast, the results of Abdul-Ghani et al. do not favor the hypothesis of increased mitochondrial oxidative stress in the development of insulin resistance. They showed in isolated mitochondria that the absolute ROS production rate is not increased in obese or diabetic subjects, but that ATP synthesis rate is reduced. As a consequence, diabetic subjects produced twice as much ROS for the same amount of ATP as obese insulin resistant and lean individuals [133]. Supporting this hypothesis, Mogensen et al. also explored the role of oxidative stress and mitochondrial capacity in skeletal muscle of T2DM subjects and age- and BMI-matched controls. In their study, mitochondrial capacity was reduced, but the oxidative stress parameter, $\mathrm{HNE}$, was similar between groups. In addition, the protein levels of UCP3, which is implicated in protection against oxidative stress, was similar between T2DM and obese controls [123].

While oxidative stress is increased in T2DM, anti-oxidants, e.g. glutathione peroxidase $3(G P X 3)$, superoxide dismutase $(S O D)$ and catalase, have been found to be down-regulated in T2DM subjects. Thiazolidiones (TZDs), which are frequently included in T2DM therapy, 


\section{Chapter 1}

can induce expression of GPX3, which results in reduced skeletal muscle $\mathrm{H}_{2} \mathrm{O}_{2}$ levels and enhanced insulin sensitivity [134]. In adipose tissue, oxidative stress directly causes insulin resistance by induction of TNFa and glucocorticoid mechanisms and reduced GLUT4 translocation [135, 136]. Moreover, fat accumulation, measured by BMI, was positively correlated with oxidative stress, and inversely correlated with plasma adiponectin levels, indicating that fat accumulation itself can induce oxidative stress, which might contribute to adipokine dysregulation [137].

In conclusion, current data suggests that elevated fatty acids can induce oxidative stress, but this is not causal for the development of insulin resistance. However, oxidative stress contributes to mitochondrial dysfunction in the diabetic state. At this moment, assessing the significance of the data on oxidative stress parameters in T2DM development is difficult, as there are a number of ways to quantify oxidative stress, a variety of biologically important reactive species, and no golden standard for determining oxidative stress or possibility to quantify oxidative stress in vivo in specific tissues or organs exists.

Mitochondrial (dys)function in diabetic skeletal muscle

Glucose entering a muscle cells via insulin-mediated uptake is subsequently converted into glycogen via glycogenesis and stored in the muscle cells, or is used to generate energy in the form of adenosine triphosphate (ATP), which can be used to drive energy requiring processes like replication, repair and growth. Glycolysis, the process by which glucose is converted into pyruvate, and the Krebs cycle generate only a few ATP molecules. The bulk of ATP is generated via the oxidative phosphorylation system (OXPHOS) in the inner membrane of the mitochondria. A number of groups have studied mitochondrial function in muscle in relation to insulin resistance and diabetes. Table 1 gives a summary of these studies, including the method (see also methods box I), sample group characteristics and the effect of correction for mitochondrial content (if performed). 


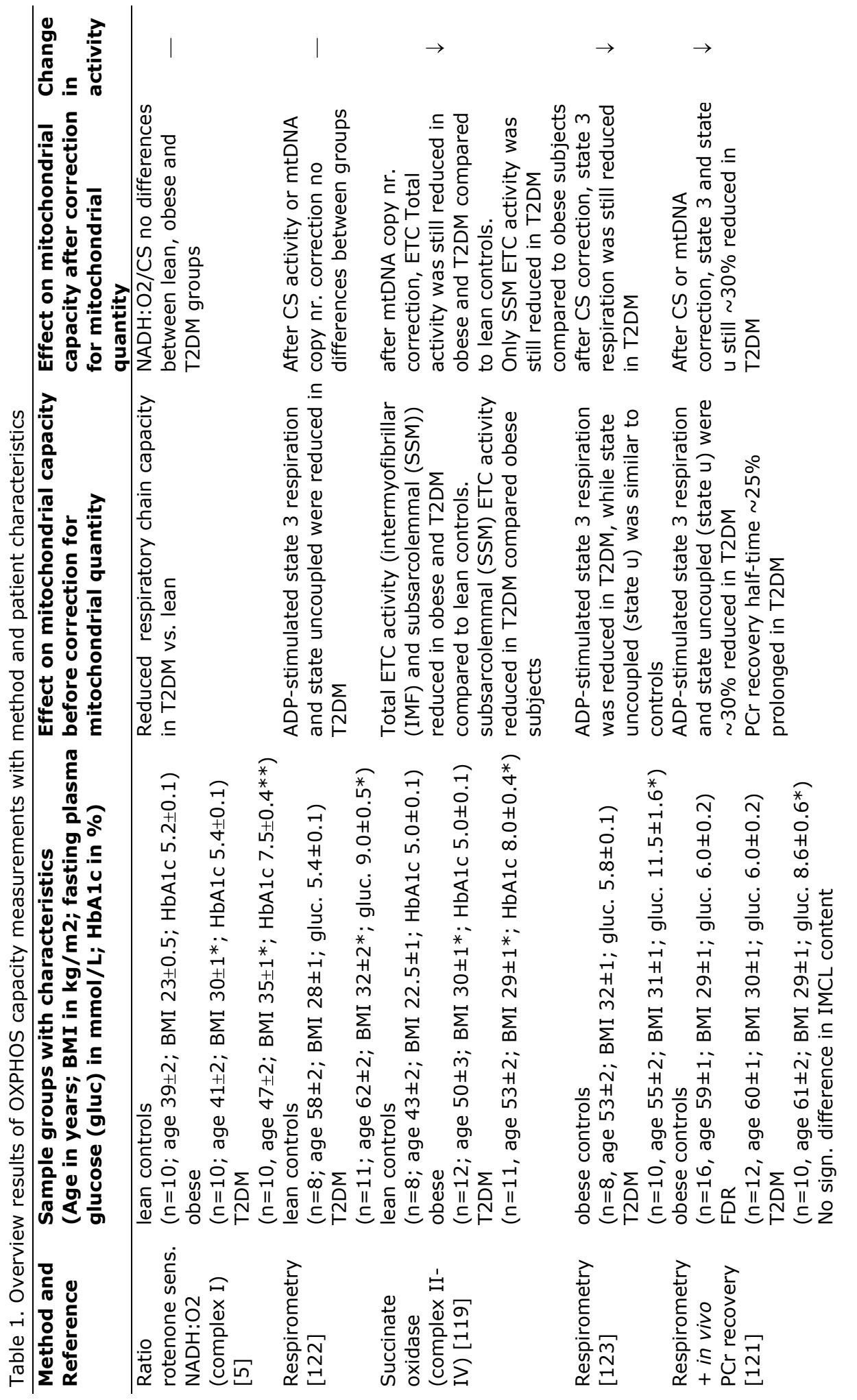




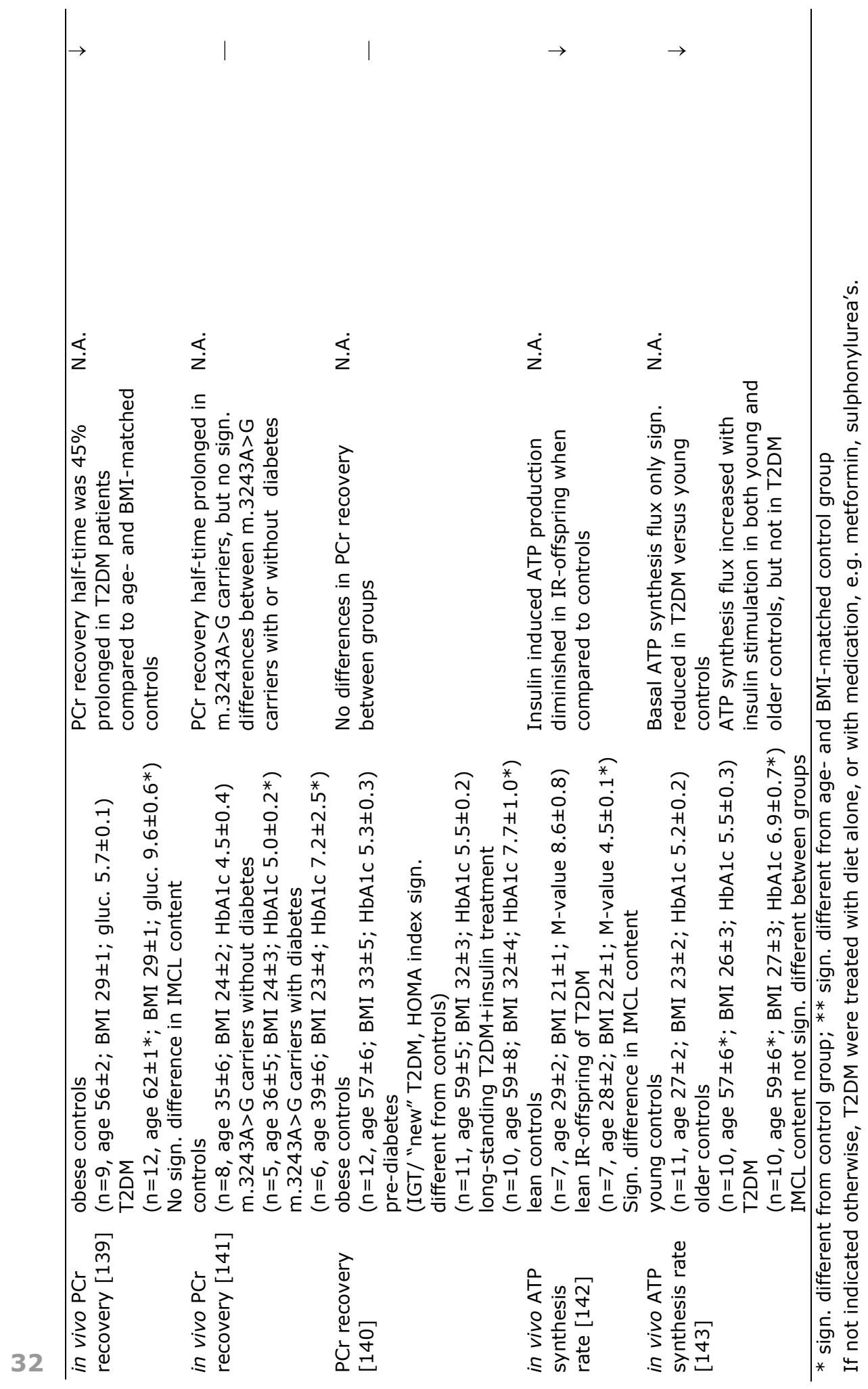




\section{Methods box I: assessment OXPHOS capacity}

Ex vivo quantification of mitochondrial OXPHOS capacity

- Mitochondrial respiration can be measured in cells and tissues using high-resolution respirometry with an Oxygraph. It contains an oxygen electrode that measures oxygen consumption in mitochondria in isotonic medium containing substrate and phosphate. By applying substrate inhibition protocols the capacity of the oxidative phosphorylation system can be evaluated. State 3 respiration is regarded as the active respiration state, while state 4 is the uncoupled state that reflects maximal electron transport chain capacity.

- Determination of rotenone-sensitive $\mathrm{NADH}: \mathrm{O} 2$ oxidoreductase activity reflects complex I function [5-7]. Spectrophotometrical analysis in intact mitochondria is used after membrane permeabilization with alamethicin to allow NADH access.

- Succinate oxidase activity represents the electron transport chain activity from complex II to IV. In isolated mitochondria succinate oxidase can be analyzed with high-performance liquid chromatography (HPLC) measurement of fumarate accumulation, which is the end-product of succinate oxidase [7].

In vivo quantification of mitochondrial OXPHOS capacity

- Mitochondrial ATP production rate in skeletal muscle can be non-invasively measured by phosphorus-31 magnetic resonance spectroscopy $\left({ }^{31} \mathrm{P}-\mathrm{MRS}\right)$. The ATP synthesis rate in basal and insulin-stimulated conditions can be calculated from the exchange between Pi and ATP.

- Phosphocreatine (PCr) can anaerobically donate a phosphate molecule to ADP to form ATP can be generated. Exercise depletes the phosphocreatine (PCr), and during recovery, the speed of $\mathrm{PCr}$ resynthesis can be quantified using ${ }^{31} \mathrm{P}-\mathrm{MRS}$ and reflects the in vivo mitochondrial function.

Difference between the two in vivo methods is that ATP synthesis rate reflects the ATP production in a given situation (basal or insulin-stimulated state) and $\mathrm{PCr}$ recovery reflects maximal ATP production capacity. Advantage of in vivo analysis is that it can be more frequently applied due to low patient burden and is not influenced by technical artifacts in sample handling or mitochondrial isolation procedures. In contrast, ex vivo analysis allows analysis of different aspects of mitochondrial functioning and density, not influenced by substrate availability, which is always a determining factor for in vivo analysis.

In the first studies, mitochondrial capacity appeared to be reduced in muscle of T2DM patients when compared with lean controls, but a number of parameters which could affect the outcome were not taken into account, like obesity, physical activity level, and mitochondrial density. In table 1, five studies are presented, each assessing muscle mitochondrial capacity ex vivo and reporting a decreased OXPHOS capacity in T2DM. However, after correcting for mitochondrial quantity (mtDNA or citrate synthase activity), the differences disappeared in two studies [5, 122], while the other three still observed down-regulated OXPHOS capacity in T2DM subjects $[119,121,123]$. From the three studies observing down-regulation, two studies found deceased mitochondrial capacity in T2DM subjects when compared with age- and BMI matched controls [121, 123], and the third study only observed decreased capacity in T2DM and age- and BMI-matched controls when 


\section{Chapter 1}

compared with lean controls [119]. These results indicated that if reduced mitochondrial capacity was observed, it was not solely due to the diabetic state, but also the obesity status appears to contribute. In the study of Mogensen et al. and Phielix et al., T2DM subjects treated with metformin and sulfonylureas, which inhibit complex I, stopped this treatment for respectively one week and one day, which could have influenced the results [138]. In addition to biological variation, differences in analytical procedures could have influenced the measurements. For instance, ex vivo analysis required muscle biopsies and analysis was performed either on isolated mitochondria or permeabilized muscle fibers. So, current ex vivo measurements do not provide sufficient evidence for an intrinsic mitochondrial defect in T2DM development.

In addition, a number of non-invasive in vivo measurements to study mitochondrial function and intramyocellular lipid (IMCL) content were performed using magnetic resonance spectroscopy (MRS). Two studies by the same group observed an increased phosphocreatin recovery (PCr) half-time in T2DM subjects when compared with age- and BMImatched controls, indicating decreased mitochondrial function in T2DM with respectively $45 \%$ and $25 \%[121,139]$. The variation in $\mathrm{PCr}$ recovery between both studies is quite large, whereas the sample groups and methods are similar, suggesting large assay variability. In contrast, de Feyter et al. did not observe a reduction with untreated prediabetic and insulin treated T2DM subjects by assessing $\mathrm{PCr}$ recovery [140]. Van Elderen et al., who studied PCr recovery in patients with mitochondrial diabetes due to the m.3243A $>G$ mutation, did not find changes in mitochondrial capacity due to the development of diabetes in these patients [141]. Since this is a specific patient group which leads to a monogenetic form of diabetes, results cannot be directly extrapolated to T2DM subjects. It merely suggests that mitochondrial dysfunction is not causal in diabetes development.

Whereas the in vivo $\mathrm{PCr}$ recovery determines the capacity of the OXPHOS system, ATP-saturation transfer methods measure the momentary flux of ATP synthesis in the resting- or insulin-stimulated state. Two studies observed a decreased ATP production rate in T2DM subjects and insulin-resistant offspring compared with lean controls $[142,143]$. The blunted ATP response in T2DM subjects with insulin- 
infusion may be derived from a reduced capacity to generate ATP, but may also be due to blunted insulin signaling or other causes [144]. In addition, base-line ATP production can also be found reduced due to decreased mitochondrial content or medication, e.g. thiozolidinediones (TZDs) like metformin inhibit complex I [138]. Major drawback in these in vivo studies is the impossibility to determine the mitochondrial density, which has been shown to have a major impact in in vitro studies, and on isolated analysis of OXPHOS activity irrespective of substrate availability. Additionally, as pointed out by De Feyter et al., the study protocol used to assess $\mathrm{PCr}$ half-time also influences the results.

Taken together, current in vivo and ex vivo data do not convincingly show that mitochondrial deficiency is causal for T2DM development. Decreased mitochondrial capacity in T2DM skeletal muscle was observed in multiple studies, but could partly be explained by decreased density rather than intrinsic mitochondrial defects. As shown in Table 1, subject characteristics largely influence the conclusions, making it difficult to compare results between studies. The conflicting results are probably due to the fact that mitochondrial function is influenced by many biological factors, such as age, BMI, IMCL content, physical activity level, medication and family history. In addition, a number of methodological issues exist, like the techniques used, analysis in isolated mitochondria or permeabilized muscle fibers, and for in vivo determination, the exercise protocol, basal vs. insulin-stimulated measurements and lack of quantification mitochondrial density.

\section{Mitochondria related gene expression changes in T2DM}

Global gene expression analysis (see methods box II) in skeletal muscle of T2DM subjects compared with normoglycemic subjects generally observed a reduced expression of genes involved in insulin signaling, transcription regulation, protein and fatty acid metabolism, mitochondrial maintenance and oxidative phosphorylation [145-148]. Insulin treatment of T2DM subjects improved transcription of genes involved in insulin signaling, transcription factors and mitochondrial maintenance, and as a result, reduced the differences in expression patterns between T2DM and control subjects [148]. Recently, Palsgaard et al. showed that insulin-resistant subjects increased expression of components of the insulin signaling pathway in skeletal muscle after a 


\section{Chapter 1}

hyperinsulinemic euglycemic clamp, while in T2DM subjects insulin signaling was down-regulated [149].

Key regulators of mitochondrial biogenesis and functioning, e.g. PGC1a and $\beta$, which are regarded as master regulators and their downstream targets NRF1, ERRa and GABPAa/b, have been found down-regulated in skeletal muscle of T2DM subjects $[145,146,150]$. PGC1a and $\beta$ expression can be induced by exercise, calcium and cold exposure and is negatively correlated with aging. As a result, down-regulation of PGC1 is not only observed in T2DM, but is also detectable in skeletal muscle of insulin resistant, obese, and inactive subjects [151, 152], so results should be interpreted carefully with respect to cause and consequence. In T2DM subjects, PGC1 expression can be induced by rosiglitazone treatment, which enhances insulin sensitivity and GLUT4 expression [153]. Since PGC1 is preferably expressed in type I fibers [154], decreased $P G C 1$ may be a contributor to the increased type IIb fiber content in insulin resistant subjects [93]. However, fiber-type shift is not the main determinant of reduced PGC1 expression, since it is also found down-regulated in T2DM compared with normoglycemic skeletal muscle samples with similar fiber-type composition [145]. Sparks et al. showed that a high-fat diet for three days reduced gene expression of OXPHOSgenes with $20-30 \%$. Additionally, expression of the key regulators of mitochondrial biogenesis, PGC1a and PGC1 $\beta$, were also $20-25 \%$ reduced in young insulin sensitive males. In contrast, the PGC1a targets NRF1 and TFAM were not affected by the high-fat diet [155], indicating that the pathways and processes involved are complex and regulated at multiple stages. Gene expression analysis in T2DM and normoglycemic age- and BMI-matched controls at equal levels of insulin and euglycemia show that altered mitochondrial function in T2DM can be attributed to insulin responsiveness rather than to an intrinsic mitochondrial defect. In T2DM, most mitochondria related genes were down-regulated but mitochondria encoded transcripts encoding subunits of electron transport chain (ETC) complexes were up-regulated at low insulin levels, which suggests compensation in T2DM subjects to maintain normal ATP production rate [124]. 
Gene expression analysis in adipose tissue of T2DM and normoglycemic subjects showed reduced expression of genes involved in the electron transport chain, citric acid cycle, ribosomal proteins and translation [156]. Expression of genes involved in mitochondrial biogenesis is downregulated in adipose tissue of $\mathrm{db} / \mathrm{db}$ mice, which are leptin-resistant due to a mutation in the leptin receptor and exhibit glucose intolerance, hyperglycemia, hyperinsulinemia, and in ob/ob mice, which are leptin deficient and have milder glucose intolerance and other metabolic symptoms. Mitochondrial biogenesis can be improved by rosiglitazone/pioglitazone treatment, which are frequently used in T2DM treatment [118, 157]. Gene expression analysis indicates decreased adipogenic capacity in adipose tissue of non-diabetic insulin resistant (IR) first-degree relatives of T2DM subjects, since key regulators of adipogenesis, e.g. peroxisome proliferator-activated receptor $\mathrm{Y}(P P A R Y)$, CCAAT/enhancer-binding proteins (C/EBPS), and sterol regulatory elements binding proteins (SREBPS) were found down-regulated. Additionally, IR subjects had enlarged adipocytes and expression of Wnt signaling genes, adiponectin and fatty acid binding protein ( $a P 2)$ were inversely correlated with adipocyte size. $[118,158]$.

\section{Methods box II: Gene expression analysis}

Differentially expressed genes between 2 or more different cell lines or tissues (for example affected versus controls) can be identified by global gene expression profiling. These genes provide information on the different ongoing molecular processes and can unravel the molecular pathology involved. Labeled amplified RNA (aRNA) of each sample is hybridized simultaneously to a large number of complementary probes, each specific for a single gene. The amount of hybridization reflects the amount of RNA and the activity of each gene. The exact procedure to perform gene expression analysis depends on the platform chosen; the two most frequently used platforms are explained here:

- The Affymetrix microarray system

This is the most widely used commercially available microarray system, which uses for each transcript 11 probes of 25 nucleotides, which hybridize specifically to a unique gene transcript, and 11 mismatch probes to measure non-specific hybridization. The Affymetrix probe-set algorithm interprets the signals from the all 22 individual probes from each probe set and derives a single value for each gene [1]. For procedure see figure 5.

- The two-color system

This system can be commercial, but is frequently a non-commercial system using home-made microarrays of spotted DNA (e.g. 70-mer oligonucleotides). For this procedure see figure 5.

Gene expression analysis generates huge amounts of data for each sample $(20,000-40,000$ signal values), which requires statistical analysis to identify significantly changed transcripts between the sample groups. Visualization and analysis of microarray data can be performed using the Gene Map Annotator and Pathway Profiler (GenMAPP) [3]. Gene expression data can be viewed within biological pathways, and the genes can be mapped based on their gene ontology into molecular function, cellular component or biological process, depending if the gene product performs respectively a molecular function, participates in a biological process or is located on or in a cellular component. Significantly overrepresented pathways and gene ontology terms can be identified based on the number of changed genes in a pathway, taking into account the size and number of analyzed genes in the pathway using the MAPPFinder tool. 


\section{Chapter 1}

Gene expression data from T2DM skeletal muscle and adipose tissue show that mitochondria-related gene expression is down-regulated in T2DM subjects, providing a molecular basis for the alterations in mitochondrial density and function observed in these subjects. However, if mitochondrial dysfunction is a consequence or a causative factor in T2DM development is unclear. When assessing several studies, patient characteristics should be kept in mind, as this can influence the results drastically. A comparison between T2DM patients and lean insulinsensitive subjects will identify alterations due to development of insulin resistance, but will also identify obesity-related processes. Likewise, different physical activity level and medication can result in different observations.

a)

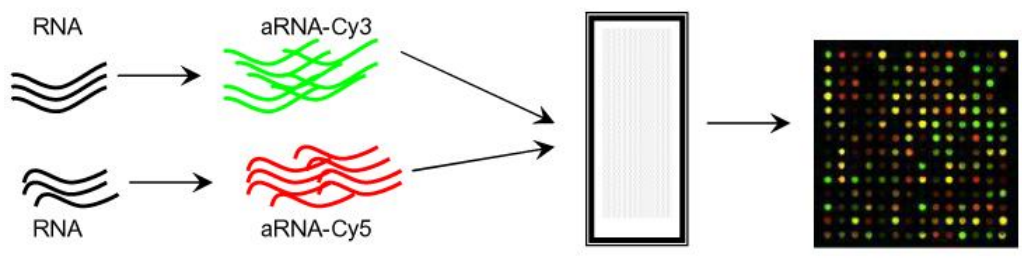

b)

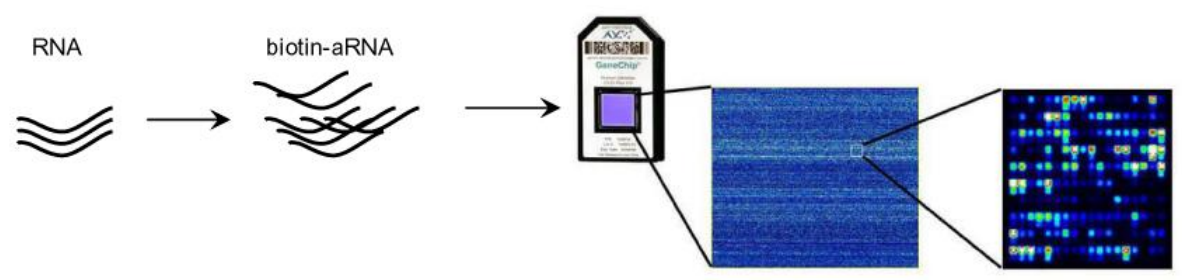

Fig 5. Schematic overview of two-color and one-color affymetrix microarray experiment

a) In a two-color experiment, microarray slides coated with $\pm 15,000$ 70-mer oligonucleotides are used. Equal amounts of two RNA samples are amplified and labeled with different fluorophores and hybridized on one microarray (one sample (e.g. control) is labeled with Cy3 (green) and sample 2 (e.g. patient) is labeled with Cy5 (red)). After hybridization and washing, the microarray is scanned at the wavelengths of both Cy 3 and Cy5. If equal amounts of a transcript are present in both samples, these transcripts will appear yellow on the array. If a transcript is overexpressed in the diseased sample labeled with Cy5, a red dot will appear. A green dot indicates overexpression in the control sample. By analyzing the $\mathrm{Cy} 5 / \mathrm{Cy} 3$ ratio, transcripts significantly over- or underrepresented in the disease state can be identified.

b) Affymetrix microarray chips contain multiple 10 -mer probes per transcript. In contrast to two-color microarrays, one amplified and biotin labeled RNA is hybridized per chip. After hybridization, the chip is stained, washed and scanned. Comparison of the absolute signal intensities between Affymetrix chips is used for identification of up- and downregulated transcripts. 
In conclusion, current data does not provide sufficient evidence that mitochondrial dysfunction has a causal role in T2DM development. However, mitochondrial function does play a central role as improving mitochondrial function has been shown to improve insulin resistance. Because of the strong dependence of T2DM on obesity and associated excessive lipid availability, mitochondria should possibly not be regarded as ATP-producing organelles, but as scavengers of the increased lipidmetabolites in the cell that inhibit insulin signaling and increase production of reactive oxygen species. Decreased capacity to metabolize the excessive lipids due to inactivity and/or intrinsic (mitochondrial) capacity may result in increased storage of harmful lipid intermediates that contribute to disease progression more than decreased ATP production rate, which might rather be a consequence. Future studies should combine data on mitochondrial capacity to oxidize glucose and lipids, amount and composition of intracellular lipids, oxidative stress level, and compare these data of T2DM subjects with controls that are matched for parameters with respect to physical activity level and free fatty acids in addition to age and BMI. Adipose tissue is most likely the first organ affected by obesity and subsequent insulin resistance, analyzing and improving adipose tissue functioning and signaling could identify, predict, and prevent disease progression at an earlier stage than when other peripheral tissues like skeletal muscle are affected.

\section{Aims and outline of this thesis}

T2DM is an energy metabolism disorder and as mitochondria are the energy factories of a cell, they have been implicated in T2DM pathophysiology. Most studies on the role of mitochondria in T2DM have focused on a single mitochondrial feature, either mitochondrial quantity, gene expression or activity. The overall aim of this thesis was to perform a comprehensive study of the contribution of mitochondria and mitochondrial dysfunction to the pathophysiology of T2DM and to determine if improvement of mitochondrial function could be beneficial for T2DM patients. This was complemented by global gene expression profiling in cells and tissues of patients to characterize new molecular pathophysiological mechanisms. 


\section{Chapter 1}

Specific aims were:

- To characterize alterations in mitochondrial function in skeletal muscle of T2DM patients.

- To characterize the pathological molecular processes in skeletal muscle and preadipocytes of patients with T2DM and primary mitochondrial diabetes by gene expression profiling.

- To characterize by in vitro experiments relevant genes and key regulators as potential targets for therapeutic interventions.

- To test the effect of exercise training on mitochondrial function and wellbeing in T2DM patients.

Chapter 1 reviews the state-of-the-art in T2DM and the role of mitochondrial energy metabolism. The picture is fragmented and heterogeneous, with a number of conflicting data sets, requiring more comprehensive investigations, as presented in this thesis. Chapter 2 describes the alterations in mitochondrial density, integrity and function in skeletal muscle tissue with the development of T2DM. After a therapeutic intervention using exercise training the same parameters have been analyzed again to measure the effect of this intervention. Additional altered processes in skeletal muscle and preadipocytes of T2DM and primary mitochondrial diabetes were identified using global gene expression analysis (Chapter 2 - 4). From these studies we selected two genes, which were specifically reduced in T2DM. The function and role of these two genes were studied in more detail in vitro (Chapter 5, 6). In the general discussion (Chapter 7), the findings and significance of the different studies were merged and discussed in a broader perspective of the pathophysiology and future treatment options for T2DM. 


\section{References}

1. Lipshutz, R.J., et al., High density synthetic oligonucleotide arrays. Nat Genet, 1999. 21 (1 Suppl): p. 20-4.

2. ADA, Diagnosis and classification of diabetes mellitus. Diabetes Care, 2005. 28 Suppl 1: p. S37-42.

3. Salomonis, N., et al., GenMAPP 2: new features and resources for pathway analysis. BMC Bioinformatics, 2007. 8: p. 217.

4. Devlieger, R., K. Casteels, and F.A. Van Assche, Reduced adaptation of the pancreatic $B$ cells during pregnancy is the major causal factor for gestational diabetes: current knowledge and metabolic effects on the offspring. Acta Obstet Gynecol Scand, 2008. 87(12): p. 1266-70.

5. Kelley, D.E., et al., Dysfunction of mitochondria in human skeletal muscle in type 2 diabetes. Diabetes, 2002. 51(10): p. 2944-50.

6. Rabol, R., R. Boushel, and F. Dela, Mitochondrial oxidative function and type 2 diabetes. Appl Physiol Nutr Metab, 2006. 31(6): p. 675-83.

7. Ritov, V.B., E.V. Menshikova, and D.E. Kelley, High-performance liquid chromatography-based methods of enzymatic analysis: electron transport chain activity in mitochondria from human skeletal muscle. Anal Biochem, 2004. 333(1): p. 27-38.

8. Eckel, R.H., S.M. Grundy, and P.Z. Zimmet, The metabolic syndrome. Lancet, 2005. 365(9468): p. 1415-28.

9. Horn, S. [cited 2009; Answers to these and other questions concerning energy metabolism, exercise and the food we eat are found here at MedBio.]. Available from:

http://www.medbio.info/Horn/IntMet/integration of metabolism\%20v4.htm.

10. Wild, S., et al., Global prevalence of diabetes: estimates for the year 2000 and projections for 2030. Diabetes Care, 2004. 27(5): p. 1047-53.

11. CDC, C.f.D.C.a.P. National diabetes fact sheet: general information and national estimates on diabetes in the United States 2007. 2008 [cited 2009 May 4th 2009]; Available from: http://www.cdc.gov/diabetes/pubs/pdf/ndfs 2007.pdf.

12. Baan, C., Poos, MJJC, Hoe vaak komt diabetes mellitus voor en hoeveel mensen sterven eraan? , in Volksgezondheid Toekomst Verkenning, Nationaal Kompas Volksgezondheid. Bilthoven. 2005, RIVM.

13. Bruggink, J., 4 percent in Dutch population suffer from diabetes mellitus. CBS Web Magazine, 2009.

14. Neckelmann, N., et al., CDNA sequence of a human skeletal muscle ADP/ATP translocator: lack of a leader peptide, divergence from a fibroblast translocator cDNA, and coevolution with mitochondrial DNA genes. Proc Natl Acad Sci U S A, 1987. 84(21): p. 7580-4.

15. Brown, W.M., M. George, Jr., and A.C. Wilson, Rapid evolution of animal mitochondrial DNA. Proc Natl Acad Sci U S A, 1979. 76(4): p. 1967-71.

16. Wallace, D.C., et al., Sequence analysis of CDNAs for the human and bovine ATP synthase beta subunit: mitochondrial DNA genes sustain seventeen times more mutations. Curr Genet, 1987. 12(2): p. 81-90.

17. Hancock, J.T., R. Desikan, and S.J. Neill, Role of reactive oxygen species in cell signalling pathways. Biochem Soc Trans, 2001. 29(Pt 2): p. 345-50.

18. Sohal, R.S. and R. Weindruch, Oxidative stress, caloric restriction, and aging. Science, 1996. 273(5271): p. 59-63.

19. Saraste, M., Oxidative phosphorylation at the fin de siecle. Science, 1999. 283(5407): p. 1488-93.

20. Heilbronn, L., S.R. Smith, and E. Ravussin, Failure of fat cell proliferation, mitochondrial function and fat oxidation results in ectopic fat storage, insulin resistance and type II diabetes mellitus. Int J Obes Relat Metab Disord, 2004. 28 Suppl 4: p. S12-21.

21. Garg, A., Acquired and inherited lipodystrophies. N Engl J Med, 2004. 350(12): p. 1220-34. 
22. Feve, B., Adipogenesis: cellular and molecular aspects. Best Pract Res Clin Endocrinol Metab, 2005. 19(4): p. 483-99.

23. Hackl, H., et al., Molecular processes during fat cell development revealed by gene expression profiling and functional annotation. Genome Biol, 2005. 6(13): p. R108.

24. Gregoire, F.M., C.M. Smas, and H.S. Sul, Understanding adipocyte differentiation. Physiol Rev, 1998. 78(3): p. 783-809.

25. MacDougald, O.A. and S. Mandrup, Adipogenesis: forces that tip the scales. Trends Endocrinol Metab, 2002. 13(1): p. 5-11.

26. Nishizuka, M., et al., Wnt4 and Wnt5a promote adipocyte differentiation. FEBS Lett, 2008. 582(21-22): p. 3201-5.

27. Kanazawa, A., et al., Wnt5b partially inhibits canonical Wnt/beta-catenin signaling pathway and promotes adipogenesis in 3T3-L1 preadipocytes. Biochem Biophys Res Commun, 2005. 330(2): p. 505-10.

28. Minokoshi, Y., C.R. Kahn, and B.B. Kahn, Tissue-specific ablation of the GLUT4 glucose transporter or the insulin receptor challenges assumptions about insulin action and glucose homeostasis. J Biol Chem, 2003. 278(36): p. 33609-12.

29. Huang, S. and M.P. Czech, The GLUT4 glucose transporter. Cell Metab, 2007. 5(4): p. 237-52.

30. Saltiel, A.R. and J.E. Pessin, Insulin signaling in microdomains of the plasma membrane. Traffic, 2003. 4(11): p. 711-6.

31. Saltiel, A.R. and C.R. Kahn, Insulin signalling and the regulation of glucose and lipid metabolism. Nature, 2001. 414(6865): p. 799-806.

32. Harigaya, S. and A. Schwartz, Rate of calcium binding and uptake in normal animal and failing human cardiac muscle. Membrane vesicles (relaxing system) and mitochondria. Circ Res, 1969. 25(6): p. 781-94.

33. Fitts, R.H. and J.J. Widrick, Muscle mechanics: adaptations with exercise-training. Exerc Sport Sci Rev, 1996. 24: p. 427-73.

34. Roden, M., Muscle triglycerides and mitochondrial function: possible mechanisms for the development of type 2 diabetes. Int J Obes (Lond), 2005. 29 Suppl 2: p. S111-5.

35. Henquin, J.C., et al., Hierarchy of the beta-cell signals controlling insulin secretion. Eur J Clin Invest, 2003. 33(9): p. 742-50.

36. Newgard, C.B. and J.D. McGarry, Metabolic coupling factors in pancreatic beta-cell signal transduction. Annu Rev Biochem, 1995. 64: p. 689-719.

37. Baggio, L.L. and D.J. Drucker, Biology of incretins: GLP-1 and GIP. Gastroenterology, 2007. 132(6): p. 2131-57.

38. Okamoto, M., et al., Adiponectin induces insulin secretion in vitro and in vivo at a low glucose concentration. Diabetologia, 2008. 51(5): p. 827-35.

39. Jensen, M.D., et al., Insulin regulation of lipolysis in nondiabetic and IDDM subjects. Diabetes, 1989. 38(12): p. 1595-601.

40. Eckel, R.H., Lipoprotein lipase. A multifunctional enzyme relevant to common metabolic diseases. N Engl J Med, 1989. 320(16): p. 1060-8.

41. Shimomura, I., et al., Leptin reverses insulin resistance and diabetes mellitus in mice with congenital lipodystrophy. Nature, 1999. 401(6748): p. 73-6.

42. Robbins, D.C., et al., Familial partial lipodystrophy: complications of obesity in the non-obese? Metabolism, 1982. 31(5): p. 445-52.

43. Horowitz, J.F., S.W. Coppack, and S. Klein, Whole-body and adipose tissue glucose metabolism in response to short-term fasting in lean and obese women. Am J Clin Nutr, 2001. 73(3): p. 517-22.

44. Kershaw, E.E. and J.S. Flier, Adipose tissue as an endocrine organ. J Clin Endocrinol Metab, 2004. 89(6): p. 2548-56.

45. Guilherme, A., et al., Adipocyte dysfunctions linking obesity to insulin resistance and type 2 diabetes. Nat Rev Mol Cell Biol, 2008. 9(5): p. 367-77.

46. Feinstein, D.L., et al., Receptor-independent actions of PPAR thiazolidinedione agonists: is mitochondrial function the key? Biochem Pharmacol, 2005. 70(2): p. 177-88. 


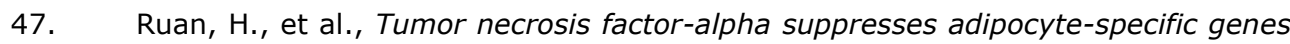
and activates expression of preadipocyte genes in 3T3-L1 adipocytes: nuclear factor-kappaB activation by TNF-alpha is obligatory. Diabetes, 2002. 51(5): p. 1319-36.

48. Sartipy, P. and D.J. Loskutoff, Monocyte chemoattractant protein 1 in obesity and insulin resistance. Proc Natl Acad Sci U S A, 2003. 100(12): p. 7265-70.

49. Yamauchi, T., et al., The fat-derived hormone adiponectin reverses insulin resistance associated with both lipoatrophy and obesity. Nat Med, 2001. 7(8): p. 941-6.

50. Curat, C.A., et al., From blood monocytes to adipose tissue-resident macrophages: induction of diapedesis by human mature adipocytes. Diabetes, 2004. 53(5): p. 1285-92.

51. Weisberg, S.P., et al., Obesity is associated with macrophage accumulation in adipose tissue. J Clin Invest, 2003. 112(12): p. 1796-808.

52. Lionetti, L., et al., From chronic overnutrition to insulin resistance: the role of fatstoring capacity and inflammation. Nutr Metab Cardiovasc Dis, 2009. 19(2): p. 146-52.

53. Schaffer, J.E., Lipotoxicity: when tissues overeat. Curr Opin Lipidol, 2003. 14(3): p. 281-7.

54. Bouzakri, K. and J.R. Zierath, MAP4K4 gene silencing in human skeletal muscle prevents tumor necrosis factor-alpha-induced insulin resistance. J Biol Chem, 2007. 282(11): p. 7783-9.

55. Stephens, J.M., J. Lee, and P.F. Pilch, Tumor necrosis factor-alpha-induced insulin resistance in 3T3-L1 adipocytes is accompanied by a loss of insulin receptor substrate-1 and GLUT4 expression without a loss of insulin receptor-mediated signal transduction. J Biol Chem, 1997. 272(2): p. 971-6.

56. Zhang, B., et al., Negative regulation of peroxisome proliferator-activated receptor-gamma gene expression contributes to the antiadipogenic effects of tumor necrosis factor-alpha. Mol Endocrinol, 1996. 10(11): p. 1457-66.

57. Puri, V., et al., RNAi screens reveal novel metabolic regulators: RIP140, MAP4k4 and the lipid droplet associated fat specific protein (FSP) 27. Acta Physiol (Oxf), 2008. 192(1): p. 103-15.

58. Menghini, R., et al., Phosphorylation of GATA2 by Akt increases adipose tissue differentiation and reduces adipose tissue-related inflammation: a novel pathway linking obesity to atherosclerosis. Circulation, 2005. 111(15): p. 1946-53.

59. Zhang, X., et al., Phosphorylation of serine 256 suppresses transactivation by FKHR (FOXO1) by multiple mechanisms. Direct and indirect effects on nuclear/cytoplasmic shuttling and DNA binding. J Biol Chem, 2002. 277(47): p. 45276-84.

60. Tamori, Y., et al., Role of peroxisome proliferator-activated receptor-gamma in maintenance of the characteristics of mature 3T3-L1 adipocytes. Diabetes, 2002. 51(7): p. 2045-55.

61. Tontonoz, P., et al., PPAR gamma 2 regulates adipose expression of the phosphoenolpyruvate carboxykinase gene. Mol Cell Biol, 1995. 15(1): p. 351-7.

62. Puri, V., et al., Fat-specific protein 27, a novel lipid droplet protein that enhances triglyceride storage. J Biol Chem, 2007. 282(47): p. 34213-8.

63. Puri, V., et al., Cidea is associated with lipid droplets and insulin sensitivity in humans. Proc Natl Acad Sci U S A, 2008. 105(22): p. 7833-8.

64. Large, V., et al., Metabolism of lipids in human white adipocyte. Diabetes Metab, 2004. 30(4): p. 294-309.

65. Granneman, J.G. and H.P. Moore, Location, location: protein trafficking and lipolysis in adipocytes. Trends Endocrinol Metab, 2008. 19(1): p. 3-9.

66. Boden, G., et al., Effects of acute changes of plasma free fatty acids on intramyocellular fat content and insulin resistance in healthy subjects. Diabetes, 2001. 50(7): p. 1612-7.

67. Watt, M.J., et al., Ciliary neurotrophic factor prevents acute lipid-induced insulin resistance by attenuating ceramide accumulation and phosphorylation of c-Jun Nterminal kinase in peripheral tissues. Endocrinology, 2006. 147(5): p. 2077-85. 
68. Goodpaster, B.H., et al., Skeletal muscle lipid content and insulin resistance: evidence for a paradox in endurance-trained athletes. J Clin Endocrinol Metab, 2001. 86(12): p. 5755-61.

69. Anderwald, C., et al., Effects of insulin treatment in type 2 diabetic patients on intracellular lipid content in liver and skeletal muscle. Diabetes, 2002. 51(10): p. 3025-32.

70. Petersen, K.F. and G.I. Shulman, Pathogenesis of skeletal muscle insulin resistance in type 2 diabetes mellitus. Am J Cardiol, 2002. 90(5A): p. 11G-18G.

71. Perseghin, G., et al., Metabolic defects in lean nondiabetic offspring of NIDDM parents: a cross-sectional study. Diabetes, 1997. 46(6): p. 1001-9.

72. Petersen, K.F., et al., Impaired mitochondrial activity in the insulin-resistant offspring of patients with type 2 diabetes. N Engl J Med, 2004. 350(7): p. 66471.

73. Dube, J.J., et al., Exercise-induced alterations in intramyocellular lipids and insulin resistance: the athlete's paradox revisited. Am J Physiol Endocrinol Metab, 2008. 294(5): p. E882-8.

74. Roden, M., et al., Mechanism of free fatty acid-induced insulin resistance in humans. J Clin Invest, 1996. 97(12): p. 2859-65.

75. Randle, P.J., et al., The glucose fatty-acid cycle. Its role in insulin sensitivity and the metabolic disturbances of diabetes mellitus. Lancet, 1963. 1(7285): p. 785-9.

76. Dresner, A., et al., Effects of free fatty acids on glucose transport and IRS-1associated phosphatidylinositol 3-kinase activity. J Clin Invest, 1999. 103(2): p. 253-9.

77. Morino, K., et al., Reduced mitochondrial density and increased IRS-1 serine phosphorylation in muscle of insulin-resistant offspring of type 2 diabetic parents. ] Clin Invest, 2005. 115(12): p. 3587-93.

78. Weyer, C., et al., Hypoadiponectinemia in obesity and type 2 diabetes: close association with insulin resistance and hyperinsulinemia. J Clin Endocrinol Metab, 2001. 86(5): p. 1930-5.

79. Hivert, M.F., et al., Associations of adiponectin, resistin, and tumor necrosis factor-alpha with insulin resistance. J Clin Endocrinol Metab, 2008. 93(8): p. 3165-72.

80. Hotta, K., et al., Plasma concentrations of a novel, adipose-specific protein, adiponectin, in type 2 diabetic patients. Arterioscler Thromb Vasc Biol, 2000. 20(6): p. 1595-9.

81. Minokoshi, Y., et al., Leptin stimulates fatty-acid oxidation by activating AMPactivated protein kinase. Nature, 2002. 415(6869): p. 339-43.

82. Tatti, P., et al., Leptin levels in diabetic and nondiabetic subjects. Endocrine, 2001. 15(3): p. 305-8.

83. Jellema, A., J. Plat, and R.P. Mensink, Weight reduction, but not a moderate intake of fish oil, lowers concentrations of inflammatory markers and PAI-1 antigen in obese men during the fasting and postprandial state. Eur J Clin Invest, 2004. 34(11): p. 766-73.

84. Kern, P.A., et al., Adipose tissue tumor necrosis factor and interleukin-6 expression in human obesity and insulin resistance. Am J Physiol Endocrinol Metab, 2001. 280(5): p. E745-51.

85. Tilg, H. and G.S. Hotamisligil, Nonalcoholic fatty liver disease: Cytokine-adipokine interplay and regulation of insulin resistance. Gastroenterology, 2006. 131(3): p. 934-45.

86. Defronzo, R.A., et al., The effect of insulin on the disposal of intravenous glucose. Results from indirect calorimetry and hepatic and femoral venous catheterization. Diabetes, 1981. 30(12): p. 1000-7.

87. Defronzo, R.A., R.C. Bonadonna, and E. Ferrannini, Pathogenesis of NIDDM. A balanced overview. Diabetes Care, 1992. 15(3): p. 318-68.

88. Lillioja, S., et al., Insulin resistance and insulin secretory dysfunction as precursors of non-insulin-dependent diabetes mellitus. Prospective studies of Pima Indians. N Engl J Med, 1993. 329(27): p. 1988-92. 
89. Kelley, D.E., et al., The effect of non-insulin-dependent diabetes mellitus and obesity on glucose transport and phosphorylation in skeletal muscle. J Clin Invest, 1996. 97(12): p. 2705-13.

90. Shulman, G.I., et al., Quantitation of muscle glycogen synthesis in normal subjects and subjects with non-insulin-dependent diabetes by $13 \mathrm{C}$ nuclear magnetic resonance spectroscopy. N Engl J Med, 1990. 322(4): p. 223-8.

91. Blaak, E.E., et al., Plasma FFA utilization and fatty acid-binding protein content are diminished in type 2 diabetic muscle. Am J Physiol Endocrinol Metab, 2000. 279(1): p. E146-54.

92. Kelley, D.E. and J.A. Simoneau, Impaired free fatty acid utilization by skeletal muscle in non-insulin-dependent diabetes mellitus. J Clin Invest, 1994. 94(6): p. 2349-56.

93. Nyholm, B., et al., Evidence of an increased number of type IIb muscle fibers in insulin-resistant first-degree relatives of patients with NIDDM. Diabetes, 1997. 46(11): p. 1822-8.

94. Oberbach, A., et al., Altered fiber distribution and fiber-specific glycolytic and oxidative enzyme activity in skeletal muscle of patients with type 2 diabetes. Diabetes Care, 2006. 29(4): p. 895-900.

95. Roden, M., Mechanisms of Disease: hepatic steatosis in type 2 diabetes-pathogenesis and clinical relevance. Nat Clin Pract Endocrinol Metab, 2006. 2(6): p. 335-48.

96. Krssak, M., et al., Alterations in postprandial hepatic glycogen metabolism in type 2 diabetes. Diabetes, 2004. 53(12): p. 3048-56.

97. Cusi, K., Nonalcoholic fatty liver disease in type 2 diabetes mellitus. Curr Opin Endocrinol Diabetes Obes, 2009. 16(2): p. 141-9.

98. Brown, M.S. and J.L. Goldstein, Selective versus total insulin resistance: a pathogenic paradox. Cell Metab, 2008. 7(2): p. 95-6.

99. Begriche, K., et al., Mitochondrial dysfunction in NASH: causes, consequences and possible means to prevent it. Mitochondrion, 2006. 6(1): p. 1-28.

100. Gentile, C.L. and M.J. Pagliassotti, The endoplasmic reticulum as a potential therapeutic target in nonalcoholic fatty liver disease. Curr Opin Investig Drugs, 2008. 9(10): p. 1084-8.

101. Li, Z., et al., The lysosomal-mitochondrial axis in free fatty acid-induced hepatic lipotoxicity. Hepatology, 2008. 47(5): p. 1495-503.

102. Kamata, H., et al., Reactive oxygen species promote TNFalpha-induced death and sustained JNK activation by inhibiting MAP kinase phosphatases. Cell, 2005. 120(5): p. 649-61.

103. Ozcan, U., et al., Endoplasmic reticulum stress links obesity, insulin action, and type 2 diabetes. Science, 2004. 306(5695): p. 457-61.

104. Sanyal, A.J., et al., Nonalcoholic steatohepatitis: association of insulin resistance and mitochondrial abnormalities. Gastroenterology, 2001. 120(5): p. 1183-92.

105. Miele, L., et al., Hepatic mitochondrial beta-oxidation in patients with nonalcoholic steatohepatitis assessed by 13C-octanoate breath test. Am J Gastroenterol, 2003. 98(10): p. 2335-6.

106. Esposito, L.A., et al., Mitochondrial disease in mouse results in increased oxidative stress. Proc Natl Acad Sci U S A, 1999. 96(9): p. 4820-5.

107. Caldwell, S.H., et al., Mitochondrial abnormalities in non-alcoholic steatohepatitis. J Hepatol, 1999. 31(3): p. 430-4.

108. Cortez-Pinto, H., et al., Alterations in liver ATP homeostasis in human nonalcoholic steatohepatitis: a pilot study. Jama, 1999. 282(17): p. 1659-64.

109. Butler, A.E., et al., Beta-cell deficit and increased beta-cell apoptosis in humans with type 2 diabetes. Diabetes, 2003. 52(1): p. 102-10.

110. Kloppel, G., et al., Islet pathology and the pathogenesis of type 1 and type 2 diabetes mellitus revisited. Surv Synth Pathol Res, 1985. 4(2): p. 110-25.

111. Muoio, D.M. and C.B. Newgard, Mechanisms of disease: molecular and metabolic mechanisms of insulin resistance and beta-cell failure in type 2 diabetes. Nat Rev Mol Cell Biol, 2008. 9(3): p. 193-205. 
112. Lee, J.W., et al., Mitochondrial dysfunction: glucokinase downregulation lowers interaction of glucokinase with mitochondria, resulting in apoptosis of pancreatic beta-cells. Cell Signal, 2009. 21(1): p. 69-78.

113. Affourtit, C. and M.D. Brand, On the role of uncoupling protein-2 in pancreatic beta cells. Biochim Biophys Acta, 2008. 1777(7-8): p. 973-9.

114. Unger, R.H., Lipotoxic diseases. Annu Rev Med, 2002. 53: p. 319-36.

115. Lukinius, A., et al., Co-localization of islet amyloid polypeptide and insulin in the $B$ cell secretory granules of the human pancreatic islets. Diabetologia, 1989. 32(4): p. 240-4.

116. Scheuner, D., et al., Control of mRNA translation preserves endoplasmic reticulum function in beta cells and maintains glucose homeostasis. Nat Med, 2005. 11(7): p. 757-64.

117. Westermark, P., et al., Islet amyloid in type 2 human diabetes mellitus and adult diabetic cats contains a novel putative polypeptide hormone. Am J Pathol, 1987. 127(3): p. 414-7.

118. Bogacka, I., et al., Pioglitazone induces mitochondrial biogenesis in human subcutaneous adipose tissue in vivo. Diabetes, 2005. 54(5): p. 1392-9.

119. Ritov, V.B., et al., Deficiency of subsarcolemmal mitochondria in obesity and type 2 diabetes. Diabetes, 2005. 54(1): p. 8-14.

120. Ortenblad, N., et al., Reduced insulin-mediated citrate synthase activity in cultured skeletal muscle cells from patients with type 2 diabetes: evidence for an intrinsic oxidative enzyme defect. Biochim Biophys Acta, 2005. 1741(1-2): p. 206-14.

121. Phielix, E., et al., Lower intrinsic ADP-stimulated mitochondrial respiration underlies in vivo mitochondrial dysfunction in muscle of male type 2 diabetic patients. Diabetes, 2008. 57(11): p. 2943-9.

122. Boushel, R., et al., Patients with type 2 diabetes have normal mitochondrial function in skeletal muscle. Diabetologia, 2007. 50(4): p. 790-6.

123. Mogensen, M., et al., Mitochondrial respiration is decreased in skeletal muscle of patients with type 2 diabetes. Diabetes, 2007. 56(6): p. 1592-9.

124. Asmann, Y.W., et al., Skeletal muscle mitochondrial functions, mitochondrial DNA copy numbers, and gene transcript profiles in type 2 diabetic and nondiabetic subjects at equal levels of low or high insulin and euglycemia. Diabetes, 2006. 55(12): p. 3309-19.

125. Ukropcova, B., et al., Family history of diabetes links impaired substrate switching and reduced mitochondrial content in skeletal muscle. Diabetes, 2007. 56(3): p. 720-7.

126. Menshikova, E.V., et al., Characteristics of skeletal muscle mitochondrial biogenesis induced by moderate-intensity exercise and weight loss in obesity. J Appl Physiol, 2007. 103(1): p. 21-7.

127. Toledo, F.G., S. Watkins, and D.E. Kelley, Changes induced by physical activity and weight loss in the morphology of intermyofibrillar mitochondria in obese men and women. J Clin Endocrinol Metab, 2006. 91(8): p. 3224-7.

128. Toledo, F.G., et al., Effects of physical activity and weight loss on skeletal muscle mitochondria and relationship with glucose control in type 2 diabetes. Diabetes, 2007. 56(8): p. 2142-7.

129. Bloch-Damti, A. and N. Bashan, Proposed mechanisms for the induction of insulin resistance by oxidative stress. Antioxid Redox Signal, 2005. 7(11-12): p. 155367.

130. Stone, J.R. and S. Yang, Hydrogen peroxide: a signaling messenger. Antioxid Redox Signal, 2006. 8(3-4): p. 243-70.

131. Bonnard, C., et al., Mitochondrial dysfunction results from oxidative stress in the skeletal muscle of diet-induced insulin-resistant mice. J Clin Invest, 2008. 118(2): p. 789-800.

132. Anderson, E.J., et al., Mitochondrial H2O2 emission and cellular redox state link excess fat intake to insulin resistance in both rodents and humans. J Clin Invest, 2009. 
133. Abdul-Ghani, M.A., et al., Mitochondrial reactive oxygen species generation in obese non-diabetic and type 2 diabetic participants. Diabetologia, 2009. 52(4): p. 574-82.

134. Chung, S.S., et al., Glutathione peroxidase 3 mediates the antioxidant effect of peroxisome proliferator-activated receptor gamma in human skeletal muscle cells. Mol Cell Biol, 2009. 29(1): p. 20-30.

135. Houstis, N., E.D. Rosen, and E.S. Lander, Reactive oxygen species have a causal role in multiple forms of insulin resistance. Nature, 2006. 440(7086): p. 944-8.

136. Rudich, A., et al., Prolonged oxidative stress impairs insulin-induced GLUT4 translocation in 3T3-L1 adipocytes. Diabetes, 1998. 47(10): p. 1562-9.

137. Furukawa, S., et al., Increased oxidative stress in obesity and its impact on metabolic syndrome. J Clin Invest, 2004. 114(12): p. 1752-61.

138. Brunmair, B., et al., Thiazolidinediones, like metformin, inhibit respiratory complex I: a common mechanism contributing to their antidiabetic actions? Diabetes, 2004. 53(4): p. 1052-9.

139. Schrauwen-Hinderling, V.B., et al., Impaired in vivo mitochondrial function but similar intramyocellular lipid content in patients with type 2 diabetes mellitus and BMI-matched control subjects. Diabetologia, 2007. 50(1): p. 113-20.

140. De Feyter, H.M., et al., Early or advanced stage type 2 diabetes is not accompanied by in vivo skeletal muscle mitochondrial dysfunction. Eur J Endocrinol, 2008. 158(5): p. 643-53.

141. van Elderen, S.G., et al., Phosphorus-31 magnetic resonance spectroscopy of skeletal muscle in maternally inherited diabetes and deafness A3243G mitochondrial mutation carriers. J Magn Reson Imaging, 2009. 29(1): p. 127-31.

142. Petersen, K.F., S. Dufour, and G.I. Shulman, Decreased insulin-stimulated ATP synthesis and phosphate transport in muscle of insulin-resistant offspring of type 2 diabetic parents. PLoS Med, 2005. 2(9): p. e233.

143. Szendroedi, J., et al., Muscle mitochondrial ATP synthesis and glucose transport/phosphorylation in type 2 diabetes. PLoS Med, 2007. 4(5): p. e154.

144. Wagenmakers, A.J., Insulin resistance in the offspring of parents with type 2 diabetes. PLoS Med, 2005. 2(9): p. e289.

145. Mootha, V.K., et al., PGC-1alpha-responsive genes involved in oxidative phosphorylation are coordinately downregulated in human diabetes. Nat Genet, 2003. 34(3): p. 267-73.

146. Patti, M.E., et al., Coordinated reduction of genes of oxidative metabolism in humans with insulin resistance and diabetes: Potential role of PGC1 and NRF1. Proc Natl Acad Sci U S A, 2003. 100(14): p. 8466-71.

147. Yang, X., et al., Microarray profiling of skeletal muscle tissues from equally obese, non-diabetic insulin-sensitive and insulin-resistant Pima Indians. Diabetologia, 2002. 45(11): p. 1584-93.

148. Sreekumar, R., et al., Gene expression profile in skeletal muscle of type 2 diabetes and the effect of insulin treatment. Diabetes, 2002. 51(6): p. 1913-20.

149. Palsgaard, J., et al., Gene expression in skeletal muscle biopsies from people with type 2 diabetes and relatives: differential regulation of insulin signaling pathways. PLoS One, 2009. 4(8): p. e6575.

150. Mootha, V.K., et al., Erralpha and Gabpa/b specify PGC-1alpha-dependent oxidative phosphorylation gene expression that is altered in diabetic muscle. Proc Natl Acad Sci U S A, 2004. 101(17): p. 6570-5.

151. Baar, K., et al., Adaptations of skeletal muscle to exercise: rapid increase in the transcriptional coactivator PGC-1. Faseb J, 2002. 16(14): p. 1879-86.

152. Benton, C.R., D.C. Wright, and A. Bonen, PGC-1alpha-mediated regulation of gene expression and metabolism: implications for nutrition and exercise prescriptions. Appl Physiol Nutr Metab, 2008. 33(5): p. 843-62.

153. Al-Khalili, L., et al., Enhanced insulin-stimulated glycogen synthesis in response to insulin, metformin or rosiglitazone is associated with increased mRNA expression of GLUT4 and peroxisomal proliferator activator receptor gamma coactivator 1. Diabetologia, 2005. 48(6): p. 1173-9. 


\section{Chapter 1}

154. Lin, J., et al., Transcriptional co-activator PGC-1 alpha drives the formation of slow-twitch muscle fibres. Nature, 2002. 418(6899): p. 797-801.

155. Sparks, L.M., et al., A high-fat diet coordinately downregulates genes required for mitochondrial oxidative phosphorylation in skeletal muscle. Diabetes, 2005. 54(7): p. 1926-33.

156. Dahlman, I., et al., Downregulation of electron transport chain genes in visceral adipose tissue in type 2 diabetes independent of obesity and possibly involving tumor necrosis factor-alpha. Diabetes, 2006. 55(6): p. 1792-9.

157. Rong, J.X., et al., Adipose mitochondrial biogenesis is suppressed in $d b / d b$ and high-fat diet-fed mice and improved by rosiglitazone. Diabetes, 2007. 56(7): p. 1751-60.

158. Yang, X., et al., Evidence of impaired adipogenesis in insulin resistance. Biochem Biophys Res Commun, 2004. 317(4): p. 1045-51. 


\section{Chapter}

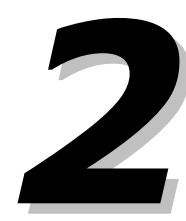

Skeletal muscle mitochondrial dysfunction is secondary to Type 2 Diabetes and can be reversed by prolonged exercise training

F.H.J. van Tienen, S.F.E. Praet, H.M. de Feyter, N.M. van den Broek, P.J. Lindsey, K.G.C. Schoonderwoerd, I.F.M. de Coo, K. Nicolay, J.J. Prompers, H.J.M. Smeets, L.J.C. van Loon 


\section{Chapter 2}

\section{Abstract}

The aim of our study is to clarify the role of mitochondrial (dys)function in the development of T2DM by a comprehensive ex vivo analysis of mitochondrial function combined with global gene expression analysis in muscle tissue. Furthermore, we assessed the effect of prolonged exercise training on mitochondrial function, performance capacity and glycemic control in long-standing insulin-treated T2DM patients.

We performed extensive ex vivo analysis of mitochondrial quality, quantity, and function combined with global gene expression analysis of skeletal muscle biopsies collected from 12 age- and BMI-matched controls, 12 pre-diabetic- and 11 long-standing T2DM subjects. In addition, in vivo ATP production capacity was assessed after a one year intrasubject controlled training intervention in long-standing T2DM patients.

Mitochondrial density, complex I activity, and expression of Krebs cycle and OXPHOS related genes were lower in long-standing T2DM subjects, but not pre-diabetic subjects compared with controls, indicating a reduced capacity to generate ATP in long-standing T2DM patients only. Gene expression analysis in pre-diabetic subjects suggested a switch from carbohydrate towards lipid as an energy source. One year exercise training raised in vivo skeletal muscle ATP production capacity by $21 \pm 2 \%$ with a trend of increased mitochondrial density and complex I activity. In addition, expression levels of $\beta$-oxidation, Krebs cycle and OXPHOS-related genes were higher following exercise training.

Mitochondrial dysfunction is a consequence rather than a cause of type 2 diabetes development. Prolonged exercise training can, at least partly, reverse the mitochondrial impairments associated with the longstanding diabetic state. 


\section{Introduction}

Type 2 diabetes mellitus (T2DM) is characterized by peripheral insulin resistance and impairments in pancreatic insulin secretion. Skeletal muscle is the critical organ, accounting for $\sim 75 \%$ of insulin-mediated glucose disposal [1]. The majority of ATP is generated by oxidative phosphorylation in the mitochondria and alterations in skeletal muscle mitochondrial quality and quantity have been observed in T2DM patients $[2,3]$. However, other studies failed to confirm these impairments [4, 5]. Similarly, in vivo and ex vivo measurements of ATP production have been reported to be reduced in skeletal muscle of T2DM patients compared with age and BMI-matched controls in some $[6,7]$, but not all studies [4, 8]. These inconsistencies could (partly) be explained by different patient characteristics and medication that either stimulate mitochondrial biogenesis or inhibit complex I activity $[9,10]$.

Mitochondrial function is negatively affected by the sedentary lifestyle many T2DM patients have adopted [11]. The lack of sufficient physical activity reduces the expression of genes involved in mitochondrial biogenesis and metabolism [12]. Reconditioning by endurance and/or resistance type exercise training represents an effective therapeutic strategy in T2DM patients, improving glycemic control, lowering blood pressure and reducing oxidative stress $[13,14]$. Physical performance capacity of T2DM patients is strongly associated with their disease status. T2DM patients with complications, such as polyneuropathy, generally display muscle weakness [15], impaired physical performance [11], poor glycemic control and a high cardiovascular risk profile [16]. These subjects are generally not advised to participate in more intense exercise intervention programs because insufficient data is available on the clinical benefits and potential health risks of such interventions. Nevertheless, prolonged resistance and interval type exercise training has been shown to augment maximal workload capacity, reduce resting blood pressure, and attenuate the progressive increase in exogenous insulin requirements in long-standing, insulin treated T2DM patients with co-morbidities [17].

Our study analyzed mitochondrial quality and quantity and global gene expression profiles in skeletal muscle from early and overt T2DM 


\section{Chapter 2}

patients compared with normoglycemic age- and BMI-matched controls. Furthermore, we also assessed the impact of prolonged exercise training on in vivo and ex vivo mitochondrial function, again in combination with global gene expression analysis, in long-standing insulin treated T2DM patients. The latter to assess whether the progression of the T2DM disease state can be attenuated and/or even reversed by exercise intervention.

\section{Methods}

Prediabetic, T2DM and control subjects

The Human Investigation Review Committee of the Máxima Medical Center (Veldhoven, The Netherlands) approved the study protocol, and the trial has been registered (ISCRTN nr.23677189). Eleven patients with long-standing ( $>5 \mathrm{yrs}$ ) T2DM, treated with exogenous insulin ( $>2$ yrs), 12 impaired glucose tolerant (IGT) pre-diabetic and newly identified T2DM subjects, and 12 normoglycemic, age- and BMI-matched controls were recruited (Table 1). All subjects were males and provided written informed consent. Control and impaired glucose tolerant subjects had no family history of T2DM and were selected based on an OGTT according to the WHO criteria [18]. Control subjects showed normal fasting glucose concentrations and glucose tolerance (fasting glucose $<6.1 \mathrm{mmol} / \mathrm{L}$ and $2 \mathrm{~h}$ glucose $<7.8 \mathrm{mmol} / \mathrm{L}$ ). Five pre-diabetic subjects had elevated fasting plasma glucose concentration (fasting glucose $\geq 6.1$ $\mathrm{mmol} / \mathrm{L}$ and $<7.0 \mathrm{mmol} / \mathrm{L}$, and $2 \mathrm{~h}$ glucose $<7.8 \mathrm{mmol} / \mathrm{L}$ ), three showed impaired glucose tolerance (fasting glucose $<7.0$ and $2 \mathrm{~h}$ glucose $\geq 7.8$ and $<11.1 \mathrm{mmol} / \mathrm{L})$ and four were recently diagnosed with T2DM $(<1$ month; fasting glucose $\geq 7.0 \mathrm{mmol} / \mathrm{L}$ or $2 \mathrm{~h}$ glucose $\geq 11.1 \mathrm{mmol} / \mathrm{L}(\mathrm{n}=4)$. None of the pre-diabetic subjects were using glucose-lowering medication and all showed glycosylated haemoglobin $\left(\mathrm{HbA}_{1 \mathrm{c}}\right)$ levels below $6.0 \%$. All T2DM patients were on exogenous insulin treatment and had been on a stable medication regimen for at least 3 months. Subjects did not have the diabetogenic m.3243A>G mutation, impaired liver function, renal failure, severe retinopathy or a history of severe cardiovascular problems. 
Table 1. Subjects' characteristics

\begin{tabular}{|c|c|c|c|c|}
\hline & \multirow[t]{2}{*}{ Controls } & \multirow[t]{2}{*}{ Pre-diabetic } & \multicolumn{2}{|c|}{ T2DM } \\
\hline & & & $t=0$ & $t=52$ \\
\hline $\mathrm{n}$ & 12 & 12 & 8 & 8 \\
\hline Age (years) & $56 \pm 6$ & $58 \pm 5$ & $60 \pm 7$ & $61 \pm 7$ \\
\hline BMI $(\mathrm{kg} / \mathrm{m} 2)$ & $32.9 \pm 4.6$ & $32.9 \pm 6.2$ & $31.7 \pm 3.3$ & $31.7 \pm 3.9$ \\
\hline Body weight (kg) & $101.0 \pm 14.7$ & $106.5 \pm 13.1$ & $96.0 \pm 15.2$ & $95.7 \pm 16.8$ \\
\hline $\mathrm{FFM}(\mathrm{kg})$ & $70.7 \pm 7.1$ & $72.4 \pm 6.9$ & $68.3 \pm 10.2$ & $68.3 \pm 10.2$ \\
\hline Truncal fat mass $(\mathrm{kg})$ & $15.5 \pm 5.5$ & $16.3 \pm 2.9$ & $15.4 \pm 3.5$ & $16.0 \pm 4.1$ \\
\hline $\mathrm{W}_{\max }(\mathrm{W})$ & $246.7 \pm 42.0$ & $245.5 \pm 29.3$ & $150.4 \pm 36.7^{b d}$ & $174.6 \pm 49.5^{\mathrm{e}}$ \\
\hline$W_{\text {max per kg } B W}(\mathrm{~W} / \mathrm{kg})$ & $2.48 \pm 0.5$ & $2.35 \pm 0.5$ & $1.61 \pm 0.5^{\mathrm{bd}}$ & $1.8 \pm 0.6^{\mathrm{e}}$ \\
\hline $\mathrm{VO}_{2 \text { peak per } \mathrm{kg} \mathrm{BW}}(\mathrm{ml} / \mathrm{min}$ per $\mathrm{kg})$ & $32.3 \pm 5.4$ & $33.0 \pm 6.3$ & $26.2 \pm 3.5^{b d}$ & $26.4 \pm 2.3$ \\
\hline $\mathrm{VO}_{2 \text { peak per } \mathrm{kg} F \mathrm{FM}}(\mathrm{ml} / \mathrm{min}$ per $\mathrm{kg})$ & $45.6 \pm 6.0$ & $47.7 \pm 8.5$ & $34.7 \pm 6.8^{b d}$ & $36.9 \pm 8.6$ \\
\hline $\mathrm{VO}_{2 \text { submax } 50 \%}$ & $2.07 \pm 0.22$ & $2.23 \pm 0.27$ & $1.73 \pm 0.18^{\mathrm{d}}$ & $1.56 \pm 0.25^{\mathrm{e}}$ \\
\hline $\mathrm{VO}_{2 \text { submax per } \mathrm{kg} \mathrm{BW}}(\mathrm{ml} / \mathrm{min}$ per $\mathrm{kg})$ & $21.3 \pm 4.4$ & $21.2 \pm 3.4$ & $18.3 \pm 2.9$ & $16.8 \pm 3.6^{\mathrm{e}}$ \\
\hline $\mathrm{VO}_{2 \text { submax per } \mathrm{kg} F \mathrm{FM}}(\mathrm{ml} / \mathrm{min}$ per $\mathrm{kg})$ & $29.9 \pm 4.4$ & $30.8 \pm 4.7$ & $25.7 \pm 3.9^{b c}$ & $23.6 \pm 4.8^{\mathrm{e}}$ \\
\hline $\mathrm{VO}_{2}$ submax per $\mathrm{kg}$ LMM $(\mathrm{ml} / \mathrm{min}$ per $\mathrm{kg})$ & $92.5 \pm 14.5$ & $99.2 \pm 14.9$ & $85.7 \pm 14.1$ & $76.6 \pm 14.5^{f}$ \\
\hline O2-pulse_submax & $17.7 \pm 2.8$ & $18.1 \pm 2.6$ & $16.0 \pm 1.8^{c}$ & $16.1 \pm 2.1$ \\
\hline Mean arterial pressure $(\mathrm{mmHg})$ & $102 \pm 9$ & $103 \pm 10$ & $104 \pm 8$ & $93 \pm 9^{f}$ \\
\hline HR steady-state submax & $118 \pm 12$ & $125 \pm 11$ & $109 \pm 14^{c}$ & $98 \pm 13^{f}$ \\
\hline $\mathrm{HR}_{\max }(\mathrm{b} / \min )$ & $166 \pm 13$ & $172 \pm 11$ & $135 \pm 25^{b d}$ & $131 \pm 25$ \\
\hline$\%$ predicted $\mathrm{HR}_{\max }$ & $99 \pm 8$ & $103 \pm 7$ & $81 \pm 14^{\mathrm{bd}}$ & $80 \pm 14$ \\
\hline Activity level (MET h/day) & $19.3 \pm 7.4$ & $19.7 \pm 8.4$ & $11.3 \pm 4.8^{\mathrm{ac}}$ & $12.5 \pm 4.1$ \\
\hline Fasting glucose (mmol/L) & $5.7 \pm 0.2$ & $6.5 \pm 0.5^{b}$ & $9.4 \pm 2.8^{\mathrm{bd}}$ & $10.8 \pm 3.7$ \\
\hline Fasting Insulin (mU/ml) & $16.2 \pm 9.1$ & $21.5 \pm 9.5$ & ND & ND \\
\hline HOMA index & $4.1 \pm 2.2$ & $6.3 \pm 2.7^{\mathrm{a}}$ & ND & ND \\
\hline 2-h glucose (mmol/L) & $5.7 \pm 1.3$ & $8.4 \pm 2.5^{\mathrm{a}}$ & ND & ND \\
\hline 2-h Insulin $(\mathrm{mU} / \mathrm{ml})$ & $74.8 \pm 40.5$ & $140.2 \pm 65^{b}$ & ND & ND \\
\hline $\mathrm{HbA}_{1 \mathrm{c}}(\%)$ & $5.3 \pm 0.3$ & $5.5 \pm 0.2$ & $7.4 \pm 0.8^{\mathrm{bd}}$ & $7.4 \pm 0.8$ \\
\hline NEFA $(\mathrm{mmol} / \mathrm{L})$ & $0.31 \pm 0.10$ & $0.40 \pm 0.12$ & $0.45 \pm 0.23^{\mathrm{a}}$ & $0.57 \pm 0.25$ \\
\hline Years with type 2 diabetes & NA & NA & $12.5 \pm 7.7$ & $13.5 \pm 7.7$ \\
\hline Years with insulin therapy & NA & NA & $8 \pm 9$ & $9 \pm 9$ \\
\hline Exogenous insulin total (IE) & NA & NA & $92.6 \pm 37.0$ & $92.0 \pm 28.1$ \\
\hline Exogenous insulin per kg (IE) & NA & NA & $0.98 \pm 0.40$ & $0.98 \pm 0.38$ \\
\hline
\end{tabular}

Significantly different from controls $\left({ }^{\mathrm{a}} \mathrm{p}<0.05,{ }^{\mathrm{b}} \mathrm{p}<0.01\right)$; significantly different from prediabetic ( $\left.{ }^{c} \mathrm{p}<0.05,{ }^{d} \mathrm{p}<0.01\right)$; significantly different from T2DM $t=0$ with paired $t$-test $\left({ }^{e} p<0.05,{ }^{f} p<0.01\right)$. Characteristics T2DM are subjects calculated for the 8 subjects that completed the 52 weeks of training to allow comparison before and after training. For cross-sectional comparison with pre-diabetic and control group, 10 T2DM subjects were analyzed, but the 8 trainings subjects are representative for the whole group $(n=10)$ and did not affect statistical analyses of differences when compared with pre-diabetic or control group. FFM, fat-free mass; $W_{\max }$, maximal workload capacity; $W_{\max }$ per $\mathrm{kg} B \mathrm{~B}, \mathrm{~W}_{\max }$ per $\mathrm{kg}$ body weight; $\mathrm{VO}_{2}$ peak, maximal oxygen uptake capacity per $\mathrm{kg}$ body weight of fat free mass; $\mathrm{VO}_{2}$ submax $50 \%$, oxygen uptake at $50 \%$ of $\mathrm{VO}_{2}$ max peak capacity; $\mathrm{VO}_{2}$ submax per LMм, $\mathrm{VO}_{\text {2submax }}$ per $\mathrm{kg}$ lean muscle mass; $\mathrm{HR}_{\max }$, maximal heart rate during the $\mathrm{VO}_{\text {peak }}$ test; \% predicted HRmax, percentage of the age-predicted $\mathrm{HR}_{\max }$; HRsteady-state submax, heart rate steady state during submax test; MET, metabolic equivalents; HOMA, homeostasis 


\section{Chapter 2}

model assessment; $2 \mathrm{~h}$ glucose/insulin, glucose/insulin concentration $2 \mathrm{~h}$ after ingestion of glucose load during the oral glucose tolerance test; $\mathrm{HbA}_{1 \mathrm{c}}$, glycosylated haemoglobin; NEFA, non-esterified fatty acids; NA, not applicable; ND, not determined.

Body composition, blood pressure and physical performance measures Body mass, waist circumference, segmental and whole-body bone and fat-free mass, systolic and diastolic blood pressure, (sub-)maximal whole-body oxygen uptake capacity $\left(\mathrm{VO}_{\text {2peak }}\right)$, workload capacity $\left(\mathrm{W}_{\max }\right)$, habitual physical activity level and quality of life were assessed as described before $[8,17]$.

\section{Training procedures, adverse events and in vivo ${ }^{31} P$ MRS analysis} All T2DM patients followed the same $1 \mathrm{yr}$ supervised exercise protocol with 5 months of progressive resistance type exercise training (PRT), supplemented with high-intensity interval exercise training (HIT) of 4-8 cycling bouts of $30 / 60 \mathrm{~s}$ at $50-60 \% \mathrm{~W}_{\max }$ [19]. Thereafter, exercise was shifted towards more endurance type exercise training with an average intensity of $79 \pm 5 \%$ of maximum heart rate frequency and an average duration of $30 \pm 6$ min per session. The latter was supplemented with $29 \pm 6$ min of PRT per session. This consisted of $2 \times 12$ reps, at $65 \pm 6 \%$ of 1 repetition maximum strength (1RM) [20], targeting 5-7 major muscle groups of the upper and lower limbs. Exercise sessions were offered 3 times a week for $1 \mathrm{yr}$. Eight of the 11 T2DM patients that started the study completed the 52 weeks exercise intervention. Three subjects stopped after 5 months, because of psychosocial (2) or medical reasons (1). In vivo analysis of ${ }^{31} \mathrm{P}$ MRS Phosphocreatine (PCr) recovery rate and ${ }^{1} \mathrm{H}$ MRS intramyocellular lipid (IMCL) content were performed 2 weeks prior to the start and 1 week after completing the training program. ${ }^{31} \mathrm{P}$ and ${ }^{1} \mathrm{H}$ MRS measurements and subsequent data analysis were performed [8]. Because of technical difficulties, ${ }^{31} \mathrm{P}$ MRS and ${ }^{1} \mathrm{H}$ MRS IMCL data from one T2DM patient were excluded from analysis.

\section{Blood analysis and ex vivo mitochondrial analysis}

In the evening, subjects received a standardized meal (mean $\pm S D$ : $41.2 \pm 15.2 \mathrm{~kJ}$ per $\mathrm{kg}$ body weight, containing 39.5 energy\% (En\%) fat, $15.1 \mathrm{En} \%$ protein and $45.4 \mathrm{En} \%$ carbohydrate), took their medication and then remained fasted. The next morning, a venous blood sample was collected after 5-10 min of supine rest. Fasting plasma glucose, non-esterified fatty acids (NEFA), serum cholesterol, HDL-cholesterol, 
LDL-cholesterol and triacylglycerol, blood glycolysated haemoglobin $\left(\mathrm{HbA}_{1 \mathrm{c}}\right)$, plasma insulin, serum adiponectin, CRP and C-peptide were analysed as described previously [17]. A percutaneous muscle biopsy was performed from the M. vastus lateralis, freed from any visible nonmuscle material, and immediately frozen in liquid nitrogen, and stored at $-80^{\circ} \mathrm{C}$. Muscle homogenates were prepared from frozen muscle and Citrate Synthase (CS), Complex I and Complex IV were measured [21, 22] and analyzed. Detailed description of the analysis is explained in electronic supplementary material (ESM) 5; All ESM can be found at http://www.personeel.unimaas.nl/florence-vantienen/, also indicated by reference [23]. DNA was isolated using the Wizard Genomic DNA isolation kit (Promega, Madison, WI, USA). Mitochondrial DNA (mtDNA) copy number was determined by real-time PCR (Applied Biosystems, Carlsbad, CA, USA). The mtDNA copy number quantification, screening for mitochondrial deletions and detection of the m.3243A>G mutation were performed using (real-time) PCR as described in ESM 5, see [23].

Gene expression analysis and real-time $P C R$ validation

Total RNA was isolated from muscle using the TRIzol reagent (Invitrogen, Carlsbad, CA, USA) and purified with the RNeasy clean-up kit (Qiagen, Hilden, Germany). Muscle RNA was amplified and hybridized on Affymetrix U133 plus 2.0 arrays, followed by staining and washing steps in the GeneChip fluidics station 400 according manufacturers' protocol (Affymetrix, Santa Clara, CA, USA). Arrays were scanned using the GeneChip scanner 3000 and quantified with GCOS software. The microarray data reported in this manuscript have been deposited in NCBI Gene expression omnibus (GEO), accession number GSE19420. Subsequent statistical analysis using multivariate Gaussian linear regression modeling has been fully described in ESM 5 [23]. The genes analyzed and fold changes were loaded into the Gene Map Annotator (GenMAPP, version 2.0) [24] and Pathway Profiler (MAPPFinder) [25] software packages. Gene database Hs-std_20070817 and MAPPs version Hs_contributed_20080619 were used to obtain a ranked list of pathways with differentially expressed genes. MAPPs with a z-score $>1.96$ and permute $p \leq 0.05$ were found significantly altered. The DAVID tool was used for identifying enriched gene ontology biological processes at level 5 , and function groups with an enrichment score $\geq 1.30(p \leq 0.05)$ were regarded as significantly changed [26]. Real-time quantitative PCR (qPCR) was used for validation. Primers (ESM 4 [23]) were designed 


\section{Chapter 2}

using Primer Express $₫$ software version 3.0 (Applied Biosystems). qPCR was performed as described [27]. The mRNA levels were normalized to the housekeeping gene for TATA-box binding protein (TBP), and results were analyzed in the same way as the microarray data (ESM 5 [23]).

\section{Statistical analysis exogenous insulin requirement}

The quantity of exogenous insulin requirements were analyzed using a multivariate Gaussian linear regression including time and a first order autocorrelation and/or a random effect to take into account the dependence among the time series of observations from the same subject. The Akaike information criterion (AIC) was used to assess whether there was a linear time trend both during the exercise training and non-training period of insulin intake (detailed description in ESM 5 [23]).

\section{Results}

Physical and ex vivo mitochondrial characteristics

HOMA-index, fasting and $2 \mathrm{~h}$ glucose and insulin levels were significantly elevated in pre-diabetic subjects when compared with the normoglycemic controls (Table 1 ). In the T2DM patients at $t=0$, peak oxygen uptake $\left(\mathrm{VO}_{2 \text { peak }}\right)$, maximal workload capacity $\left(\mathrm{W}_{\max }\right)$, maximal heart rate $\left(H R_{\max }\right)$ and activity level (MET $h$ /day) were significantly lower than the other 2 groups. In addition, non-esterified fatty acid content, and fasting glucose and insulin levels were higher when compared with controls and pre-diabetic subjects. Mitochondrial density (CS) and complex I per CS were significantly reduced in the T2DM patients, and complex IV per CS was slightly elevated in both pre-diabetic and T2DM subjects (Table 2). MtDNA copy number was comparable for control, pre-diabetic and T2DM groups $(3201 \pm 719,3598 \pm 742$, and $3607 \pm 622$ (mean \pm SD), respectively). Six of the 12 controls had no mtDNA deletion, 3 had a single deletion and 3 had multiple deletions. In the 12 prediabetic subjects, no deletion was detectable in half of the group, 4 subjects had a single deletion, and 2 subjects had multiple deletions. Finally, in the T2DM subjects, 5 out of 9 had no deletion, 1 a single deletion and 3 multiple deletions. 


\section{Mitochondrial dysfunction in T2DM}

Table 2. Alterations in OXPHOS activity pre-T2DM and T2DM compared with controls, and after 52 weeks of training in T2DM group.

\begin{tabular}{lccc}
\hline & $\begin{array}{c}\text { pre-T2DM } \begin{array}{c}(\mathrm{n}=12) \\
\text { vs. Controls } \\
(\mathrm{n}=11)\end{array} \\
\text { CS / mg protein }\end{array}$ & $\begin{array}{c}\text { T2DM }(\mathrm{n}=7) \text { vs. } \\
\text { Controls }(\mathrm{n}=11)\end{array}$ & $\begin{array}{c}\text { T2DM } \mathrm{t}=52 \text { vs. } \mathrm{t}=0 \\
(\mathrm{n}=6)\end{array}$ \\
Complex I / CS & $0.86(0.73-1.00)$ & $0.70(0.57-0.85)^{\mathrm{a}}$ & $1.14(0.96-1.35)$ \\
Complex I / mg protein & $0.83(0.67-1.02)$ & $0.54(0.42-0.70)^{\mathrm{a}}$ & $1.26(1.00-1.61)$ \\
Complex IV / CS & $1.11(0.53-0.91)^{\mathrm{a}}$ & $0.36(0.26-0.50)^{\mathrm{a}}$ & $1.39(1.10-1.74)^{\mathrm{b}}$ \\
Complex IV / mg protein & $0.96(0.82-1.12)$ & $0.90(0.74-1.17)$ & $1.05(0.88-1.61)$ \\
\hline
\end{tabular}

Data is expressed as mean fold change ( \pm C.I.). The Akaike information criterion was used for statistical modeling. ${ }^{\text {a }}$ Significantly changed compared with the control group; ${ }^{b}$ significantly changed in T2DM group at t52 compared to T2DM at t0.

Table 3. ${ }^{31} \mathrm{P}$ MRS parameters during rest, end of exercise and recovery in 7 insulin-treated T2DM patients before and after 52 weeks of exercise training.

\begin{tabular}{llcc}
\hline & & T2DM t $=0$ & T2DM t=52 \\
\hline Rest & $\mathrm{pH}$ & $7.06 \pm 0.02$ & $7.08 \pm 0.01^{\mathrm{a}}$ \\
& {$[\mathrm{PCr}](\mathrm{mM})$} & $36.6 \pm 4.4$ & $36.4 \pm 2.2$ \\
& {$[\mathrm{Pi}](\mathrm{mM})$} & $4.5 \pm 0.8$ & $4.8 \pm 0.3$ \\
& {$[\mathrm{ADP}](\mathrm{uM})$} & $10.1 \pm 0.4$ & $10.5 \pm 0.3^{\mathrm{a}}$ \\
& {$[\mathrm{PDE}](\mathrm{mM})$} & $6.2 \pm 1.4$ & $5.72 \pm 1.4^{\mathrm{a}}$ \\
End-exercise & $\mathrm{pH}$ & $6.9 \pm 0.12$ & $6.94 \pm 0.06$ \\
& {$[\mathrm{PCr}](\mathrm{mM})$} & $20.3 \pm 3.9$ & $19.2 \pm 2.8$ \\
& {$[\mathrm{Pi}](\mathrm{mM})$} & $19.8 \pm 3.5$ & $19.5 \pm 2.5$ \\
Recovery & {$[\mathrm{ADP}](\mathrm{uM})$} & $46.4 \pm 11.8$ & $53.5 \pm 11.0^{\mathrm{a}}$ \\
\hline & $\mathrm{K}_{\mathrm{PCr}}(1 / \mathrm{min})$ & $1.33 \pm 0.44$ & $1.63 \pm 0.65^{\mathrm{a}}$ \\
\hline
\end{tabular}

Data is expressed as mean \pm S.D.; PCr, phosphocreatine; Pi, inorganic phosphate; ADP, adenosine diphosphate; $\mathrm{pH}$, intracellular muscle $\mathrm{pH} ; \mathrm{K}_{\mathrm{PCr}}, \mathrm{PDE}$, phosphodiesters; $\mathrm{PCr}$ recovery rate constant. Significantly changed from $t=0$ analyzed with paired $t$-test, ${ }^{a}$ $\mathrm{p}<0.05$.

Table 4. Serum analysis before and after training of T2DM subjects

\begin{tabular}{lcc}
\hline & T2DM t=0 & T2DM t=52 \\
\hline Adiponectin $(\mathrm{mg} / \mathrm{l})$ & $2.13 \pm 0.88$ & $2.24 \pm 0.81$ \\
C-peptide $(\mathrm{nmol} / \mathrm{l})$ & $0.60 \pm 0.16$ & $0.66 \pm 0.20$ \\
CRP $(\mathrm{mg} / \mathrm{l})$ & $1.77 \pm 0.58$ & $1.73 \pm 0.33$ \\
FFA $(\mu \mathrm{mol} / \mathrm{l})$ & $478 \pm 69$ & $569 \pm 75$ \\
Triacylglycerol $(\mathrm{mmol} / \mathrm{l})$ & $1731 \pm 148$ & $2503 \pm 431$ \\
Total cholesterol $(\mathrm{mmol} / \mathrm{l})$ & $4.09 \pm 0.18$ & $4.50 \pm 0.29$ \\
HDL cholesterol $(\mathrm{mmol} / \mathrm{l})$ & $0.83 \pm 0.07$ & $0.96 \pm 0.06^{\mathrm{a}}$ \\
LDL cholesterol $(\mathrm{mmol} / \mathrm{l})$ & $3.34 \pm 0.14$ & $2.40 \pm 0.23^{\mathrm{b}}$ \\
HbA1c $(\%)$ & $7.36 \pm 0.24$ & $7.43 \pm 0.26$ \\
\hline
\end{tabular}

Data is expressed as mean $\pm S D ; n=8 . ;$ Significantly changed from $t=0$ analyzed with paired t-test, ${ }^{\mathrm{a}} \mathrm{p}<0.05,{ }^{\mathrm{b}} \mathrm{p}<0.01$ 


\section{Chapter 2}

Global gene expression analysis in muscle

A total of 1,707 genes were differentially expressed with a fold-change of $>10 \%$ (ESM 1 [23]), 153 genes were differently expressed in both the pre-diabetic and T2DM group compared with controls, 550 specifically in the pre-diabetic subjects and 851 in the T2DM patients. In the pre-diabetic group an equal number of genes were up- and downregulated, but in the T2DM group the majority (70\%) was decreased. Fold changes were in general small. In the pre-diabetic group the highest fold changes were 0.57 and 1.93, in the T2DM group 0.43 and 1.91. qPCR analysis of 11 genes showed a significant fold change in the same direction for 8 genes, but 3 genes only a non-significant trend (ESM 4, see [23]).

Annotated differently expressed genes were used for gene ontology analysis (GenMAPP/MAPPFinder program; Table ESM 2, see [23]) to identify altered pathways (Table 5). Next, we used DAVID for gene ontology analysis of biological processes. For the pre-diabetic group, 2 functional groups were significantly enriched, namely GO terms associated with glucose and steroid metabolism (ESM 3a, see [23]). In the T2DM group, 6 functional groups were significantly enriched, including Krebs cycle, protein metabolism and transport, nucleic acid metabolism and epigenetic regulators (ESM 3b, see [23]). In general, DAVID confirmed the MAPPFinder processes, adding processes not included in the local MAPPs of MAPPFinder, e.g. chromatin modifiers, $\mathrm{NAD}^{+} / \mathrm{NADH}$ recycling, tRNA synthesis and aminoacetylation and amino acid synthesis.

Stage-specific alterations in energy metabolism related gene expression In the pre-diabetic group, genes involved in the initial breakdown of glucose to pyruvate in the glycolysis were decreased compared with controls. In contrast, fatty acid $\beta$-oxidation, fatty acid and triglyceride synthesis were increased, suggesting a switch from glucose towards fatty acids as an energy source (Table 5). Genes of the striated muscle contraction pathway encoding components of type I or oxidative fibers were up-regulated in pre-diabetic subjects, while expression of type II glycolytic muscle fiber components was lower. 


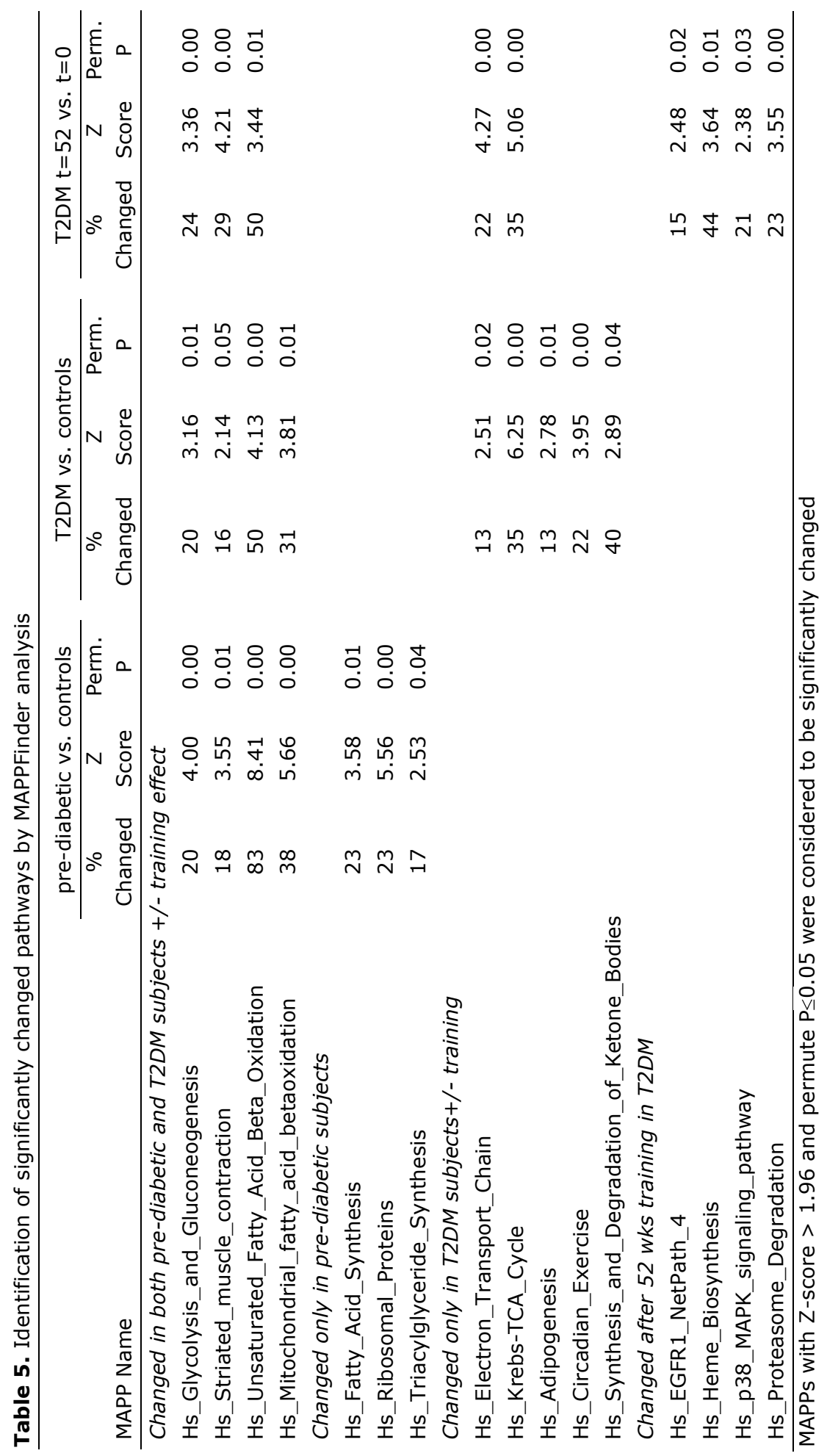




\section{Chapter 2}

Genes involved in glycolysis did not differ between T2DM and prediabetic subjects. However, expression of genes involved in substrate transport into mitochondria, conversion of pyruvate into acetyl-CoA, and the aspartate-malate shuttle, which recycles NADH/NAD ${ }^{+}$used for ATP synthesis in the mitochondria, was lower in the T2DM patients. Besides a lower ability to utilize glucose as an energy source, expression of genes involved in Krebs cycle and electron transport chain was lower in T2DM patients. The latter might impair the capacity to use fatty acids for energy generation. In addition, increased ketone body synthesis by upregulation of $H M G C S 2$, and decreased ketone body metabolism reflected by decreased expression of the key regulators $O X C T 1$ and $B D H$ were observed in T2DM patients.

\section{Processes specifically altered in T2DM subjects}

Other processes were also altered in the T2DM group (Table 5). Circadian rhythm components are regulated in a time- and exercisedependent manner and play a role in various processes, including transcription, cell-cycle regulation and glucose uptake [28]. In addition, a number of genes were altered in the adipogenesis pathway, which contains genes involved in the transcriptional regulation of adipogenesis and cell-cycle regulation, but also in lipid storage and energy metabolism.

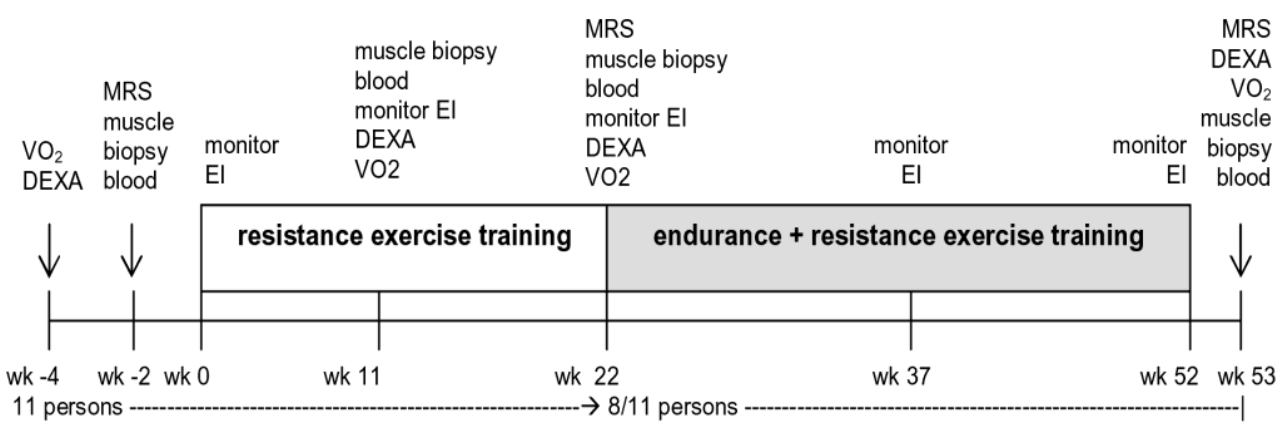

Fig 1. Overview of the study protocol, including time-points of sample/data collection. monitor EI, monitor exogenous insulin requirement; MRS, ${ }^{31} \mathrm{P}$ and ${ }^{1} \mathrm{H}$ MRS measurements of mitochondrial function and IMCL content respectively; VO2, oxygen uptake at peak and $50 \%$; DEXA, dual energy X-ray absorptiometry to assess body composition and fat free mass. 
Increased physical performance and mitochondrial capacity after 52 weeks of exercise training

Eight T2DM subjects completed a 52 weeks exercise training protocol (Fig. 1). They participated on average in 1.9 \pm 0.4 (mean \pm SD) exercise sessions per week, representing $62 \pm 13 \%$ of all sessions. After 52 weeks, maximal workload capacity $\left(\mathrm{W}_{\max }\right)$, submaximal oxygen uptake and heart rate improved significantly compared with baseline measurements (Table 1). No significant differences in quality of life were observed with scores of $61 \pm 24$ and $65 \pm 23$ (mean \pm SD), respectively, on the RAND36 scale of $0-100$. No significant changes in glycemic control were identified, but exogenous insulin (EI) requirements were significantly reduced after 52 weeks of training when compared with the expectations derived from the 3 preceding years ( +0.80 units $\mathrm{EI} / \mathrm{month}$ ) (Figure 2).

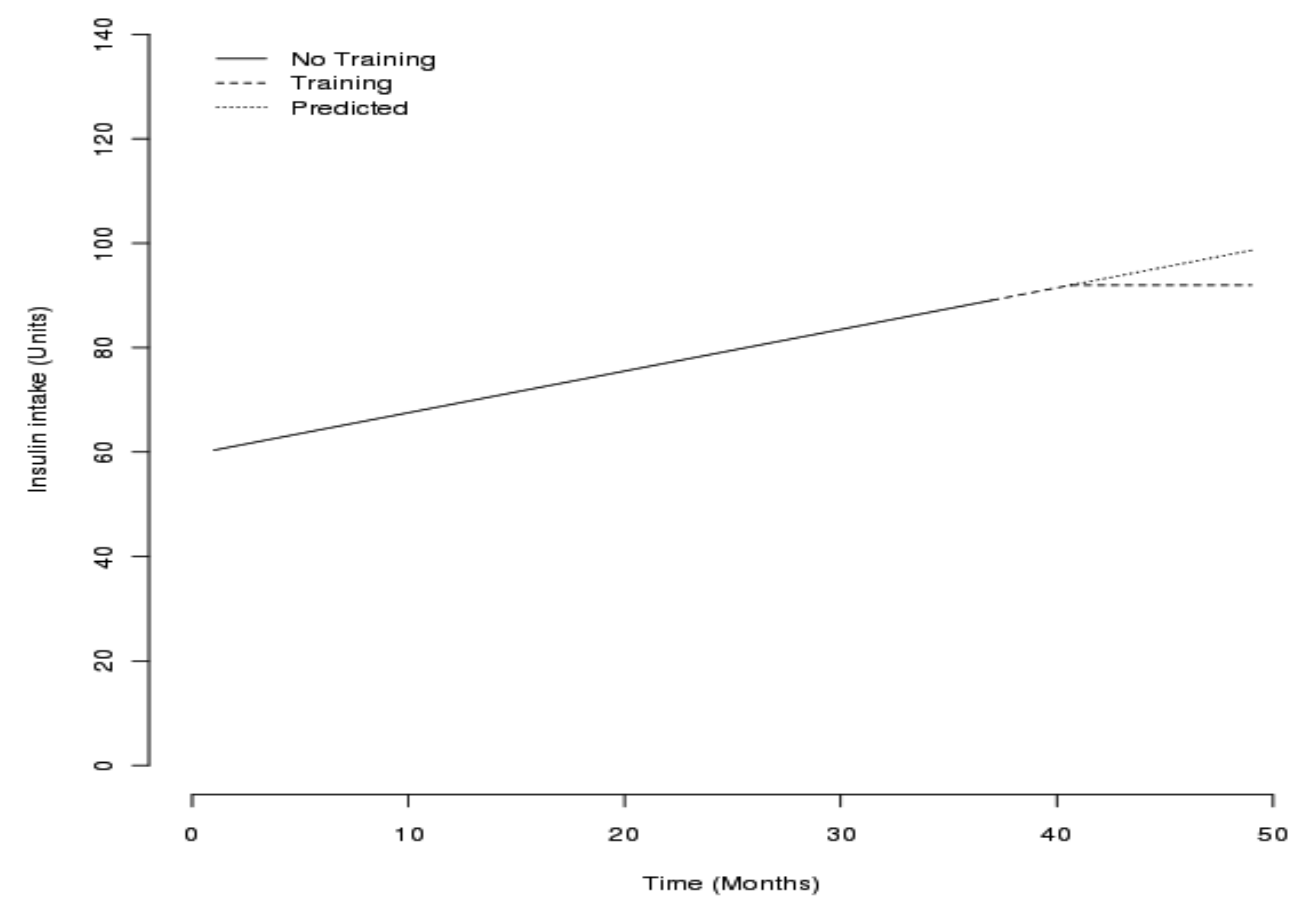

Fig. 2. Exogenous insulin requirements of T2DM patients prior to and during the exercise training period.

The solid line represents the mean exogenous insulin (EI) requirements of the eight longstanding T2DM subjects before participating in the exercise training study, and the EI requirements during the 52 weeks of exercise training, from month 36 till 48, indicated by dashed line. The dotted line represents the expected increase in EI requirement without exercise training, which was calculated based on the EI increase prior to participation in the training protocol. 


\section{Chapter 2}

In vivo ${ }^{31} \mathrm{P}$ MRS measurements showed that exercise training significantly increased the $\mathrm{PCr}$ recovery rate constant $(21 \pm 2 \%)$, which is a measure for oxidative capacity (Table 3 ). ${ }^{1} \mathrm{H}$ MRS measurements showed no changes in IMCL content. Ex vivo analysis of citrate synthase activity and Complex I activity/CS activity ratio showed no significantly increase, but complex I activity/protein ratio was significantly higher after 52 weeks of exercise training (Table 2). Complex IV activity remained unchanged. In addition, mtDNA copy number at $\mathrm{t}=0$ and $\mathrm{t}=52$ were $3662 \pm 641$ and $3513 \pm 1023$ (mean \pm SD) respectively, indicating no significant differences in mitochondrial content. Finally, no differences in large-scale mtDNA deletions were observed.

The effect of 52 weeks exercise training on skeletal muscle gene expression

After 52 weeks of training, 1,095 genes were altered in T2DM subjects; 387 were decreased and 708 increased (ESM 1 [23]). The highest fold changes were 0.59 and 3.58 . Some altered pathways were the same as in pre-diabetic/T2DM patients compared with controls, but others were specific for exercise training (Table 5, ESM 2 [23]). In addition, 6 GO biological process terms related to Krebs cycle, electron transport, protein transport, heme biosynthesis, cell growth and (negative) regulation of apoptosis were altered (ESM 3c [23]). While the majority of genes involved in energy metabolism were reduced in long-term T2DM patients, training significantly enhanced expression of a number of genes in electron-transport chain, Krebs cycle, fatty acid $\beta$-oxidation and glycolysis (Fig. 3, and Table 5).

Prolonged exercise training altered the expression profile of the striated muscle contraction pathway towards the pre-diabetic subjects. The training also increased the EGFR1 and heme biosynthesis pathway, both of which are involved in a wide range of cellular processes, like differentiation, proliferation and transcription/translation cell-cycle regulation. In contrast, the catabolic proteasome degradation pathway was decreased after training. 


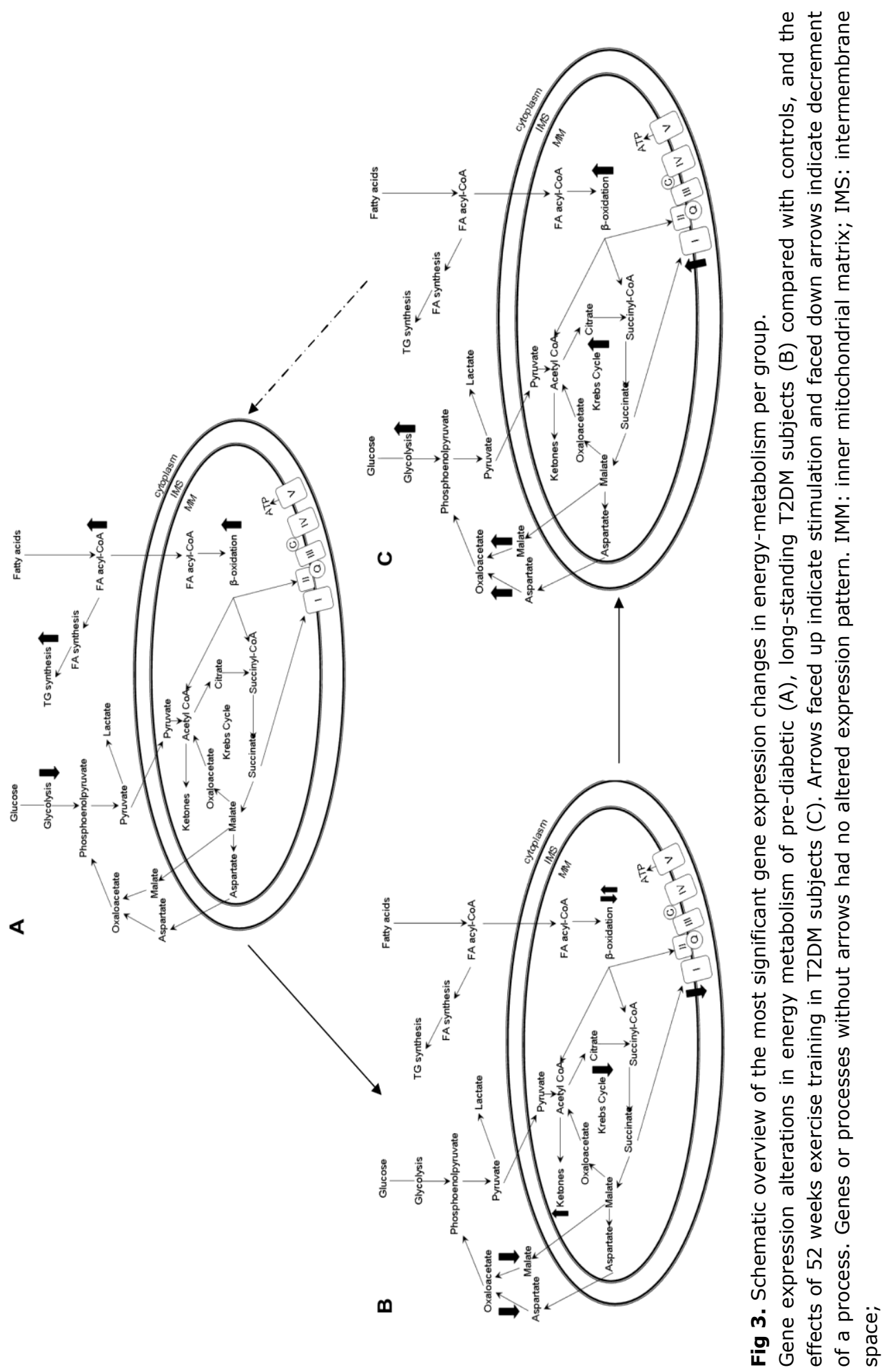




\section{Chapter 2}

\section{Discussion}

The present study shows that mitochondrial dysfunction is prevalent in long-standing insulin treated T2DM patients and not in pre-diabetic patients, putting mitochondrial dysfunction secondary to diabetes development (Fig. 3). Moreover, gene expression analysis implies an increased use of fatty acids for energy provision in pre-diabetic subjects, which is subsequently abolished in insulin-treated T2DM subjects. The insulin-treated T2DM patients are physically less active than the other groups, show less oxidative capacity and a lower expression of Krebs cycle and electron transport chain components. After a year of regular exercise training, the expression profiles in the T2DM subjects were partly reversed, now resembling the gene expression profile as observed in the pre-diabetic subjects (Fig. 3). Prolonged exercise training improved in vivo and ex vivo mitochondrial function as well as global gene expression analysis, indicative of an increased capacity to metabolize fat for energy generation.

Mitochondrial dysfunction is secondary to T2DM development

Cross-sectional comparison of pre-diabetic subjects, T2DM patients, and age- and BMI-matched controls allowed us to analyze mitochondrial function and integrity at different stages of diabetes development. We did not observe an increase in the amount of mtDNA deletions and excluded the presence of the m.3243A $>\mathrm{G}$ mutation in the pre-diabetic and T2DM patients. Therefore, we conclude that mtDNA integrity is comparable among the different groups, but a different prevalence of point-mutations cannot be excluded. Citrate Synthase (CS) activity, a marker for mitochondrial density, was significantly lower in the T2DM group, whereas no differences in mtDNA copy number were present between groups. In addition to CS activity, complex I activity per mitochondrion was also specifically reduced in the T2DM patients. These changes in mitochondrial capacity were supported by reduced gene expression levels in the Krebs cycle and electron transport chain pathways. The absence of impairments in mitochondrial function, decreases in mitochondrial density and/or mitochondria related gene expression in pre-diabetic subjects indicated that mitochondrial dysfunction has no primary, causal role in diabetes development. It is 
more likely a consequence of the T2DM state and associated decrement in physical activity.

Over the last few years, a number of studies have reported mitochondrial function, reflected by reduced ATP production capacity, to be compromised in T2DM patients when compared to age- and BMI matched controls $[6,29]$. In contrast, others have failed to detect any impairments in muscle tissue of T2DM patients [4]. Biological and technical factors may (partly) be responsible for these discrepancies. Biologically, mitochondrial function can be influenced by physical activity status and the use of medication, e.g. metformin [9]. Technically, analysis of mitochondrial function requires correction for mitochondrial density and should be based on an independent and pure marker of mitochondrial function. Additional evidence that mitochondrial dysfunction is not a prerequisite for the development of insulin resistance and/or T2DM is provided by the disproportionately large capacity for mitochondrial ATP-generation [30] and the prevalence of enhanced mitochondrial function in diabetic Asian Indians [31]. Furthermore, mitochondrial respiratory chain defects that result in severely decreased ATP production in skeletal muscle tissue are generally not accompanied by T2DM development. One exception is the mtDNA m.3243A>G mutation [32], but development of diabetes in these subjects is not associated with a further reduction in in vivo $\mathrm{PCr}$ recovery rate [33]. Taken together, impaired mitochondrial function does not seem to represent a cause, but rather a consequence of T2DM development.

This could be determined in our study, in contrast to others, by including both a group of newly identified and a group of long-standing T2DM subjects. Moreover, the observed impairments may not merely be the result of T2DM development. It could also be attributed to a reduced physical activity level [12], chronic use of metformin [9, 10] and persisting hyperglycemia [34], despite exogenous insulin therapy, which is known to improve mitochondrial function $[5,34]$.

Ritov et al. showed a reduction in thickness of the subsarcolemmal mitochondrial layer and electron transport chain activity in T2DM subjects [7]. Subsarcolemmal mitochondria provide energy for membrane-related processes, play a key role in fatty acid metabolism and account for one third of the ATP production [7, 35]. Like most other 


\section{Chapter 2}

studies, we could not detect these location-specific impairments when analyzing mixed muscle homogenates containing both subsarcolemmal and intramyofibrillar (IMF) mitochondria. However, the observed higher complex IV/CS activity in pre-diabetic and T2DM subjects might indirectly indicate a greater proportion of IMF fraction in the homogenate [36]. This observation favors that mitochondrial content is lower due to a reduced subsarcolemmal area [7], but studies applying electron microscopy are warranted to confirm this hypothesis.

\section{Lipid handling capacity plays a key role in T2DM etiology}

In the pre-diabetic subjects, reduced expression was observed for genes involved in glucose metabolism, but increased expression of genes in lipid metabolism. This activation was subsequently abolished in longstanding T2DM patients (Fig. 3). This supports that mitochondrial function is not impaired in the pre-diabetic state, allowing greater fat use for energy generation $[37,38]$. Increased $\beta$-oxidation is likely a rescue mechanism to avoid the detrimental effects of increased lipid(intermediates) on insulin signaling, like in obese normoglycemic subjects [38]. The increase in $\beta$-oxidation disappeared in full-blown T2DM and was associated with increased formation of ketone bodies and IMCL content [8]. Our data emphasizes that mitochondria are more than just ATP producers. Insufficient sequestering of fatty acids by the mitochondria results in increased storage of lipids and lipid intermediates, without affecting the in vivo ATP production capacity. Further study of the relation between IMCL content, lipid-intermediates and mitochondrial function should provide important knowledge on disease progression and possible intervention targets.

\section{The effects of long-term interval exercise training}

The long-standing insulin-treated T2DM subjects showed decreased muscle strength, aerobic capacity and a lower activity level, and were unable to participate in a more generic endurance exercise training intervention program designed to treat chronic metabolic disease. A combined resistance and interval type exercise training program has been proven feasible and more beneficial for this patient subgroup [17]. To study the long-term effects, 8 subjects completed a 52 week training protocol. T2DM patients increased muscle strength, submaximal aerobic capacity and in vivo and ex vivo mitochondrial function. Gene expression analysis showed stimulation of fatty acid $\beta$-oxidation, Krebs cycle and 
OXPHOS-related genes, also normally stimulated by exercise training in healthy controls [39]. These processes were specifically decreased in the T2DM subjects compared to controls. The exercise-induced stimulation indicated that the right processes were targeted, being beneficial for the T2DM patients. For example, NAD+ regeneration capacity was reduced in untrained T2DM patients but was increased after training, like in prediabetic subjects. In addition, training also increased glucose metabolizing capacity, similar to exercise training of metabolic syndrome patients [40]. The positive influence of exercise training on mitochondrial function without a change in body composition, further confirms that mitochondrial function is not only influenced by obesity, but also inactivity $[12,41]$.

The 52 weeks of exercise training increased PGC1a expression, a key regulator of mitochondrial function and biogenesis. PGC1a has been reported to be down-regulated in T2DM, but is also decreased as a result of obesity and inactivity [42]. Increased PGC1a expression by exercise training in obese rats has been shown to increase fatty acid $\beta$ oxidation and enable mitochondria to cope better with increased fatty acids [43]. Because of the key regulatory function of $P G C 1 a$, a number of changes observed after training were likely a result of PGC1a activation. Training also improved blood lipid-profiles of T2DM subjects, but glycemic control was not changed with respect to changes in fasting glucose or HbA1c. However, their exogenous insulin requirements stabilized in contrast to the increasing requirements in the 3 preceding years (Fig. 2). Since we did not observe changes in C-peptide, the stabilization of exogenous insulin requirements can be attributed to the adaptive response to training on whole-body insulin sensitivity, e.g. by decreasing hepatic glucose output, increasing muscle mass, increasing glycogen and intramuscular lipid storage capacity, and improving skeletal muscle perfusion. In line with this, a recent 12-week training intervention of well-controlled metformin-treated T2DM subjects showed that exercise training restored metabolic flexibility, and increased muscle strength, mitochondrial density and activity, with only a slight improvement in insulin-mediated glucose depositioning [44]. Finally, gene expression analysis indicated reduced muscle wasting and increased muscle regeneration, especially for type I muscle fiber, which possibly contributes to increased fatty acid oxidation. Exercise training 


\section{Chapter 2}

may, therefore, reverse the loss of oxidative fibers in metabolic syndrome and T2DM $[45,46]$.

In summary, our most important findings in skeletal muscle of prediabetic and insulin-treated T2DM subjects are: (1) mitochondrial dysfunction is only apparent in long-standing T2DM patients and should, as such, be regarded a consequence rather than a cause of T2DM development, (2) mitochondrial dysfunction in long-standing T2DM patients on exogenous insulin therapy can, at least partly, be reversed by prolonged endurance and resistance type exercise training.

\section{Acknowledgements}

The authors would like to thank J. Senden, S. Vanherle and I. Eijkenboom for technical assistance, and P. Rietjens, J. Swolfs, I. Jansen, L. van den Meijdenberg and N. van Herpen for their assistance and supervising the exercise intervention program. The research presented was funded by grants from the Ministry of Health, Welfare and Sport and the Dutch Diabetes Research Foundation (DFN 2004.00.040). 


\section{References}

1. Stump, C.S., et al., The metabolic syndrome: role of skeletal muscle metabolism. Ann Med, 2006. 38(6): p. 389-402.

2. Ortenblad, N., et al., Reduced insulin-mediated citrate synthase activity in cultured skeletal muscle cells from patients with type 2 diabetes: evidence for an intrinsic oxidative enzyme defect. Biochim Biophys Acta, 2005. 1741(1-2): p. 206-14.

3. Kelley, D.E., et al., Dysfunction of mitochondria in human skeletal muscle in type 2 diabetes. Diabetes, 2002. 51(10): p. 2944-50.

4. Boushel, R., et al., Patients with type 2 diabetes have normal mitochondrial function in skeletal muscle. Diabetologia, 2007. 50(4): p. 790-6.

5. Asmann, Y.W., et al., Skeletal muscle mitochondrial functions, mitochondrial DNA copy numbers, and gene transcript profiles in type 2 diabetic and nondiabetic subjects at equal levels of low or high insulin and euglycemia. Diabetes, 2006. 55(12): p. 3309-19.

6. Phielix, E., et al., Lower intrinsic ADP-stimulated mitochondrial respiration underlies in vivo mitochondrial dysfunction in muscle of male type 2 diabetic patients. Diabetes, 2008.

7. Ritov, V.B., et al., Deficiency of subsarcolemmal mitochondria in obesity and type 2 diabetes. Diabetes, 2005. 54(1): p. 8-14.

8. De Feyter, H.M., et al., Early or advanced stage type 2 diabetes is not accompanied by in vivo skeletal muscle mitochondrial dysfunction. Eur J Endocrinol, 2008. 158(5): p. 643-53.

9. Brunmair, B., et al., Thiazolidinediones, like metformin, inhibit respiratory complex I: a common mechanism contributing to their antidiabetic actions? Diabetes, 2004. 53(4): p. 1052-9.

10. Feinstein, D.L., et al., Receptor-independent actions of PPAR thiazolidinedione agonists: is mitochondrial function the key? Biochem Pharmacol, 2005. 70(2): p. 177-88.

11. Sayer, A.A., et al., Type 2 diabetes, muscle strength, and impaired physical function: the tip of the iceberg? Diabetes Care, 2005. 28(10): p. 2541-2.

12. Timmons, J.A., et al., Expression profiling following local muscle inactivity in humans provides new perspective on diabetes-related genes. Genomics, 2006. 87(1): p. $165-72$.

13. Santeusanio, F., et al., Diabetes and exercise. J Endocrinol Invest, 2003. 26(9): p. $937-40$.

14. Nojima, $\mathrm{H}_{.}$, et al., Effect of aerobic exercise training on oxidative stress in patients with type 2 diabetes mellitus. Metabolism, 2008. 57(2): p. 170-6.

15. Andersen, $\mathrm{H}_{\text {., }}$ et al., Muscle strength in type 2 diabetes. Diabetes, 2004. 53(6): p. $1543-8$.

16. Wei, M., et al., Low cardiorespiratory fitness and physical inactivity as predictors of mortality in men with type 2 diabetes. Ann Intern Med, 2000. 132(8): p. 60511.

17. Praet, S.F., et al., Long-standing, insulin-treated type 2 diabetes patients with complications respond well to short-term resistance and interval exercise training. Eur J Endocrinol, 2008. 158(2): p. 163-72. 


\section{Chapter 2}

18. World Health Organization and International Diabetes Federation, Definition and diagnosis of diabetes mellitus and intermediate hyperglycaemia report of a WHO/IDF consultation. WHO Press. 2006, Geneva.

19. De Feyter, H.M., et al., Exercise training improves glycemic control in longstanding insulin-treated type 2 diabetic patients. Diabetes Care, 2007. 30(10): p. 2511-3.

20. Reynolds, J.M., T.J. Gordon, and R.A. Robergs, Prediction of one repetition maximum strength from multiple repetition maximum testing and anthropometry. J Strength Cond Res, 2006. 20(3): p. 584-92.

21. Scholte, H.R., et al., Riboflavin-responsive complex I deficiency. Biochim Biophys Acta, 1995. 1271(1): p. 75-83.

22. van Empel, V.P., et al., Downregulation of apoptosis-inducing factor in harlequin mutant mice sensitizes the myocardium to oxidative stress-related cell death and pressure overload-induced decompensation. Circ Res, 2005. 96(12): p. e92e101.

23. Van Tienen, F.H. Supplementary material thesis. 2010 [cited; Available from: http://www.personeel.unimaas.nl/florence-vantienen/.

24. Salomonis, N., et al., GenMAPP 2: new features and resources for pathway analysis. BMC Bioinformatics, 2007. 8: p. 217.

25. Doniger, S.W., et al., MAPPFinder: using Gene Ontology and GenMAPP to create a global gene-expression profile from microarray data. Genome Biol, 2003. 4(1): p. R7.

26. Dennis, G., Jr., et al., DAVID: Database for Annotation, Visualization, and Integrated Discovery. Genome Biol, 2003. 4(5): p. P3.

27. van Tienen, F.H., et al., Wnt5b stimulates adipogenesis by activating PPARgamma, and inhibiting the beta-catenin dependent Wnt signaling pathway together with Wnt5a. Biochem Biophys Res Commun, 2009. 387(1): p. 207-11.

28. Zambon, A.C., et al., Time- and exercise-dependent gene regulation in human skeletal muscle. Genome Biol, 2003. 4(10): p. R61.

29. Mogensen, M., et al., Mitochondrial respiration is decreased in skeletal muscle of patients with type 2 diabetes. Diabetes, 2007. 56(6): p. 1592-9.

30. Holloszy, J.O., Skeletal muscle "mitochondrial deficiency" does not mediate insulin resistance. Am J Clin Nutr, 2009. 89(1): p. 463S-6S.

31. Nair, K.S., et al., Asian Indians have enhanced skeletal muscle mitochondrial capacity to produce ATP in association with severe insulin resistance. Diabetes, 2008. 57(5): p. 1166-75.

32. $\mathrm{t}$ Hart, L.M., et al., Heteroplasmy levels of a mitochondrial gene mutation associated with diabetes mellitus decrease in leucocyte DNA upon aging. Hum Mutat, 1996. 7(3): p. 193-7.

33. van Elderen, S.G., et al., Phosphorus-31 magnetic resonance spectroscopy of skeletal muscle in maternally inherited diabetes and deafness A3243G mitochondrial mutation carriers. J Magn Reson Imaging, 2009. 29(1): p. 127-31.

34. Sreekumar, R., et al., Gene expression profile in skeletal muscle of type 2 diabetes and the effect of insulin treatment. Diabetes, 2002. 51(6): p. 1913-20.

35. Koves, T.R., et al., Subsarcolemmal and intermyofibrillar mitochondria play distinct roles in regulating skeletal muscle fatty acid metabolism. Am J Physiol Cell Physiol, 2005. 288(5): p. C1074-82. 


\section{Mitochondrial dysfunction in T2DM}

36. Cogswell, A.M., R.J. Stevens, and D.A. Hood, Properties of skeletal muscle mitochondria isolated from subsarcolemmal and intermyofibrillar regions. Am J Physiol, 1993. 264(2 Pt 1): p. C383-9.

37. Mogensen, M., et al., Maximal lipid oxidation in patients with type 2 diabetes is normal and shows an adequate increase in response to aerobic training. Diabetes Obes Metab, 2009. 11(9): p. 874-83.

38. Perseghin, G., et al., Normal insulin sensitivity and IMCL content in overweight humans are associated with higher fasting lipid oxidation. Am J Physiol Endocrinol Metab, 2002. 283(3): p. E556-64.

39. Earnest, C.P., Exercise interval training: an improved stimulus for improving the physiology of pre-diabetes. Med Hypotheses, 2008. 71(5): p. 752-61.

40. Hittel, D.S., et al., Exercise training increases electron and substrate shuttling proteins in muscle of overweight men and women with the metabolic syndrome. J Appl Physiol, 2005. 98(1): p. 168-79.

41. Szendroedi, J., et al., Muscle mitochondrial ATP synthesis and glucose transport/phosphorylation in type 2 diabetes. PLoS Med, 2007. 4(5): p. e154.

42. Patti, M.E., et al., Coordinated reduction of genes of oxidative metabolism in humans with insulin resistance and diabetes: Potential role of PGC1 and NRF1. Proc Natl Acad Sci U S A, 2003. 100(14): p. 8466-71.

43. Koves, T.R., et al., Peroxisome proliferator-activated receptor-gamma coactivator 1alpha-mediated metabolic remodeling of skeletal myocytes mimics exercise training and reverses lipid-induced mitochondrial inefficiency. J Biol Chem, 2005. 280(39): p. 33588-98.

44. Meex, R.C., et al., Restoration of muscle mitochondrial function and metabolic flexibility in type 2 diabetes by exercise training is paralleled by increased myocellular fat storage and improved insulin sensitivity. Diabetes. 59(3): p. 5729.

45. Gaster, M., et al., GLUT4 is reduced in slow muscle fibers of type 2 diabetic patients: is insulin resistance in type 2 diabetes a slow, type 1 fiber disease? Diabetes, 2001. 50(6): p. 1324-9.

46. Nyholm, B., et al., Evidence of an increased number of type IIb muscle fibers in insulin-resistant first-degree relatives of patients with NIDDM. Diabetes, 1997. 46(11): p. 1822-8. 



\section{Chapter}

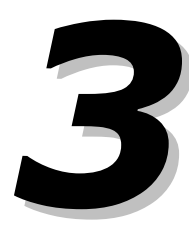

Gene expression profiling reveals differences in pathological processes in skeletal muscle of affected carriers of the m.3243A>G (MELAS) mutation

F.H.J. van Tienen, P.J. Lindsey, S.C.E.H. Salleveld, S.G. Frints, I.F.M. de Coo, V. Tiranti, H.J.M. Smeets 


\section{Chapter 3}

\section{Abstract}

The m.3243A>G mutation of the mitochondrial tRNALeu is one of the most frequent mtDNA mutations. It can give rise to mitochondrial encephalopathy with lactic acidosis and stroke-like episodes (MELAS), but additionally or separately also to a number of other clinical manifestations, like diabetes, hearing loss and cardiomyopathy. The mutation percentage only partly explains the clinical heterogeneity. Therefore, we performed gene expression profiling in a group of 8 patients with this mutation and 3 controls to identify processes, which could explain the variety of clinical manifestations. Unsupervised hierarchical clustering of all genes with alterations larger than median gene variability grouped two m.3243A>G patients with healthy controls (cluster 1). The second cluster contained the other 6 patients. Subsequent process analysis of genes differently expressed between the two clusters indicated a reduced expression of genes involved in aerobic energy generation in the second cluster. In addition, increased proapoptotic gene expression and altered expression of processes implicated in cell proliferation and muscle regeneration were observed, indicating increased cellular stress. This data indicates that therapies directed at maintaining muscle regenerative capacity may slow down disease progression. The 2 patients, who clustered with the controls in cluster 1 , were the youngest patients with the highest mutation load. Both suffered from diabetes, but neuromuscular manifestations were mild. As no clear gene expression differences were observed in muscle between the controls and these patients, this may indicate that the diabetes does not primarily result from pronounced muscle alterations, but more likely from pancreatic $\beta$-cell dysfunction. Studying pancreatic $\beta$-cell and skeletal muscle of mitochondrial diabetes patients will increase our understanding of insulin resistance and mitochondrial malfunctioning in common type 2 diabetes patients. 


\section{Introduction}

A large number of mitochondrial DNA (mtDNA) mutations have been described, giving rise to a broad variety of clinical manifestations. One of the most common mtDNA mutations is the m.3243A>G mutation in the dihydrouridine loop of mitochondrial tRNA ${ }^{\text {Leu(UUR) }}$ [1]. As a result of the mutation, the tertiary structure of the tRNA leucine changes and leads to a general reduction of mitochondrial protein synthesis $[2,3]$. The majority of m.3243A>G carriers develop mitochondrial myopathy, encephalopathy, lactic acidosis and stroke-like episodes (MELAS), but the mutation can in addition or separately lead to other phenotypes like hypertrophic cardiomyopathy, hearing loss, or renal failure. In addition, nearly all m.3243A $>$ G carriers will have developed diabetes by the age of 70 years, although the age at onset varies considerably. Accordingly, the m.3243A $>\mathrm{G}$ is regarded as a highly diabetogenic mutation [4]. In general, major clinical symptoms are correlated with muscle m.3243A>G mutation load, e.g. encephalopathies are detected at younger age in m.3243A $>G$ carriers with a high mutation load [5]. However, clinical presentation is highly variable [6] and no exact correlation between the m.3243A>G mutation percentage, clinical phenotype $[7,8]$, haplogroup or additional polymorphisms have been observed [9]. Such a correlation does exist for some other mtDNA mutations, like the m.8993T>G mutation [10]. So far, skeletal muscle mutation load has only been correlated with development of diabetes and hearing loss in a single study [11], and clearly, other factors are involved in determining nature and severity of clinical and other phenotypic manifestations.

In a previous study by our group, gene expression profiling was used to identify altered processes in affected and unaffected m.3243A>G carriers. Unaffected carriers induced processes implicated in protein turnover and metabolic adaptation to compensate for the genetic defect. Development of mild symptoms in m.3243A>G carriers was associated with loss of these compensatory processes and activation of muscle regeneration by complement activation. The number of differentially expressed genes between affected m.3243A>G carriers and controls was strikingly lower than between asymptomatic carriers and controls [12]. Since the m.3243A $>\mathrm{G}$ mutation leads to a wide range of clinical 


\section{Chapter 3}

manifestations, different processes may be distinguishable within the group of symptomatic m.3243A $>\mathrm{G}$ carriers that are missed when comparing with only unaffected m.3243A>G carriers. Therefore, we applied gene expression profiling combined with unsupervised hierarchical clustering as an unbiased approach to cluster 8 affected female m.3243A>G carriers and three female controls. The significantly altered processes between the groups were related to the phenotypic manifestations in each of the groups.

\section{Materials and Methods}

\section{Subjects}

Our study included three female controls and eight female m.3243A>G carriers with a mutation load $>10 \%$ in skeletal muscle. A muscle biopsy from the $M$. vastus lateralis, taken from each person under local anesthesia, was immediately frozen in liquid nitrogen. All phenotypic characteristics of the m.3243A $>$ G carriers are presented in Table 1. The control group consisted of three healthy females of 38, 39 and 59 years old without the m.3243A>G mutation.

\section{Quantification of m.3243A>G mutation percentage}

DNA was isolated from the skeletal muscle samples using the DNA cleanup wizard kit (Promega) according to the manufacturer's protocol. To amplify the fragment carrying the m.3243A>G mutation by PCR, forward 5'-CAACTTAGTATTATACCCACAC-3' and reverse primer 5'ATTACGAATGGCTTGCTTT-3' were used, and a FAM-labelled primer in the last PCR cycle. Heteroplasmy levels were determined by mutation specific restriction digestion with the HaeIII enzyme resulting in a wildtype fragment of 128 bases and a mutant fragment of 56 bases long. Digested products were analyzed on an ABI-PRISM 3100 genetic analyzer using the GeneScan Analysis 3.7 software package (Applied Biosystems, Foster City - CA, USA). The ratio of the area under the mutant peak and the sum of the areas under the mutant and wild-type peaks were calculated to determine the heteroplasmy levels of the m.3243A>G mutation. 
Table 1. Characteristics m.3243A $>$ G carriers

\begin{tabular}{|c|c|c|c|c|c|c|c|c|}
\hline m.3243A>G ID nr. & 15 & 19 & 31 & 33 & 34 & 35 & 52 & 53 \\
\hline Cluster & 2 & 2 & 2 & 1 & 2 & 1 & 2 & 2 \\
\hline Age at diagnosis (years) & 32 & 32 & 50 & 27 & 55 & 26 & 31 & 54 \\
\hline \multicolumn{9}{|l|}{ Age at muscle biopsy } \\
\hline (years) & 33 & 40 & 51 & 29 & 56 & 27 & 31 & 54 \\
\hline Stroke & no & no & no & no & no & no & & \\
\hline Seizures & no & no & no & no & no & no & & \\
\hline Lactic acidosis & no & no & & no & & & & \\
\hline Focal brain lesions (CT/MRI) & no & yes & & & & no & & \\
\hline Headache/migraine & no & yes & yes & no & no & yes & & \\
\hline BG calcifications & no & & & & & no & & \\
\hline Hearing loss & yes & yes & yes & yes & yes & yes & yes & yes \\
\hline Diabetes & no & no & no & yes & no & yes & no & yes \\
\hline Ophtalmoparesis & yes & yes & no & no & no & no & & \\
\hline \multirow[t]{2}{*}{ Family history } & yes & yes & yes & yes & yes & yes & yes & yes \\
\hline & 31 & & 15 & 34,35 & 33,35 & 33,34 & 53 & 52 \\
\hline \multirow[t]{2}{*}{ Short stature* } & no & yes & & yes & & no & & \\
\hline & $-1,5 \mathrm{SD}$ & $-2 S D$ & & $-2,5 \mathrm{SD}$ & & $-1 S D$ & & \\
\hline Weakness & yes & yes & no & $\begin{array}{l}\text { yes } \\
\text { (mild) }\end{array}$ & no & $\begin{array}{l}\text { yes } \\
\text { (mild) }\end{array}$ & & \\
\hline Cardiomyopathy & yes & yes & no & \pm & \pm & \pm & & \\
\hline Ataxia & yes & yes & no & no & no & no & & yes \\
\hline Mental retardation & yes & \pm & no & \pm & no & no & & \\
\hline Swallow problems & no & no & no & no & no & no & & \\
\hline Facial weakness & yes & \pm & no & \pm & no & no & & \\
\hline Diffuse cerebellar atrophy & no & no & & & & no & & \\
\hline Cognitive disorder & yes & no & no & no & no & no & & \\
\hline Tired & yes & yes & no & yes & yes & yes & & yes \\
\hline Retinitis pigmentosa & yes & yes & & yes & yes & & & \\
\hline Opticus atrophy & yes & no & & no & & & & \\
\hline Ptosis & yes & yes & no & no & yes & no & & \\
\hline Conduction defect (ECG) & yes & yes & \pm & no & no & no & & \\
\hline Renal disease & no & & no & yes & no & no & yes & \\
\hline RRF & yes & & no & no & yes & no & no & yes \\
\hline COX-fibers & & & yes & yes & yes & no & no & yes \\
\hline Morph. alterations muscle & yes & & yes & yes & yes & no & yes & yes \\
\hline A3243G muscle (\%) & 32 & 61 & 27 & 76 & 54 & 74 & 59 & 58 \\
\hline A3243G blood (\%) & 15 & 15 & 5 & 32 & 6 & 30 & & \\
\hline Complex I activity & & $41 \%$ & & & & & & \\
\hline
\end{tabular}

\pm indicates border-line; $*$ shorter than $2 *$ SD is defined as short stature 


\section{Chapter 3}

Gene expression analysis

Total RNA was isolated using the TRIzol reagent (Invitrogen, Carlsbad CA, USA) and purified with the RNeasy clean-up kit (Qiagen, Hilden Germany) according to the manufacturer's protocol. RNA quantity and purity were determined spectrophotometrically using the Nanodrop ND1000 (Nanodrop Technologies, Wilmington - DE, USA) and RNA integrity was assessed by determining the RNA 28S/18S ratio using the Bioanalyzer 2100 (Agilent Technologies, Santa Clara - CA, USA). Muscle RNA spiked with four bacterial RNA transcripts was reverse transcribed into CDNA and amplified in a two-round amplification reaction according to the manufacturer's protocol (Affymetrix, Santa Clara - CA, USA). A mixture of CDNA and added hybridization controls was hybridized on Affymetrix U133 plus 2.0 arrays, followed by staining and washing steps in the GeneChip fluidics station 400 (Affymetrix) according to the manufacturer's procedures. To assess the raw probe signal intensities, chips were scanned using the GeneChip scanner 3000 (Affymetrix).

\section{Statistical analysis}

Normalization and gene expression summaries were computed for each probe set using robust multichip analysis (RMA). In addition, all transcripts showing variability significantly smaller than the median gene variability were removed $\left(P<0.01\right.$, based on the $\chi^{2}$ test implemented in $\mathrm{BRB}$ array tools version 3.8.0). All transcripts that passed the selection criteria were used for unsupervised hierarchical clustering with average linkage.

The chip description file (CDF) used for the analysis was an update created and freely distributed by the microarray lab of the university of Michigan [13, 14] based on Ensembl (version 10). The genes were analyzed using a Gaussian linear regression including the chip's log mean intensity and the mutation percentage. The inference criterion used for comparing the models is their ability to predict the observed data, i.e. models are compared directly through their minimized minus log-likelihood. When the numbers of parameters in models differ, they are penalized by adding the number of estimated parameters, a form of the Akaike information criterion (AIC) [15]. For each gene, the group was then added to the model. The gene under consideration was found to be differentially expressed if the AIC decreased compared to the model not containing the group effect, the confidence interval did not 
include a fold change of 1 , the fold change increase or decrease was at least $10 \%$, and the mean signal intensity was $>200$ in both groups or $>300$ in one group.

Visualization and analysis of microarray data on biological pathways was performed using the Gene Map Annotator and Pathway Profiler (GenMAPP, version 2.0) [16]. Gene database Hs-std_20070817 and MAPPs version Hs_contributed_20080619 were used. Significantly changed pathways were identified using the MAPPFinder program, which expresses a z-score, permute-p and adjusted-p for each pathway using Fisher's exact test. Pathways with a $z$-score $>1.96$ and permute $P \leq 0.05$ were considered to be significant [17]. The DAVID tool was used for identifying enriched gene ontology biological processes at level 5, and function groups with an enrichment score $\geq 1.30$ ( $p \leq 0.05$ ) were regarded as significantly changed [18].

\section{Real-time quantitative $P C R$ validation}

Differentially expressed genes were validated by real-time quantitative PCR (qPCR) on the same RNA samples as used for the microarrays. Primers were designed using Primer Express $₫$ software version 3.0 (Applied Biosystems), (sequences primers, see electronic supplementary material (ESM) 4, also indicated with [19]). cDNA was synthesized using Superscript II cDNA synthesis kit (Invitrogen). Quantification was carried out using the ABI HT7900 Real-Time PCR detection system using qPCR Sensimix for SYBR Green I (Quantace). The cycling conditions were: an initial step for $2^{\prime}$ at $50^{\circ} \mathrm{C}$, activation of the enzyme at $95^{\circ} \mathrm{C}$ for $10^{\prime}$, and 40 cycles of $15^{\prime \prime}$ at $95^{\circ} \mathrm{C}$ followed by $1^{\prime}$ at $60^{\circ} \mathrm{C}$. The mRNA levels of each gene were normalized to those of the housekeeping gene encoding TATA-box binding protein (TBP), which was invariable between the muscle samples. Results were analyzed using a Gaussian linear regression, similar to the microarray analysis. The housekeeping gene and age were included in the analysis. The AIC was used to assess whether there was a difference between cluster 1 and cluster 2. All statistical analyses presented were performed using the freely available program R [20] and the publicly available library 'growth' [21]. 
Microarray analysis U133 plus 2.0

3 healthy controls \& 8 m.3243A $>$ G carriers

Unsupervised clustering using 13,645 transcripts

with variability larger than median gene variability,

$P<0.01$, based on the $\chi^{2}$ test

Cluster 1

young mildly affected $\mathrm{m} .3243 \mathrm{~A}>\mathrm{G}$ and controls

\section{Cluster 2}

young more severely affected and older mildly affected

m.3243A $>\mathrm{G}$ carriers

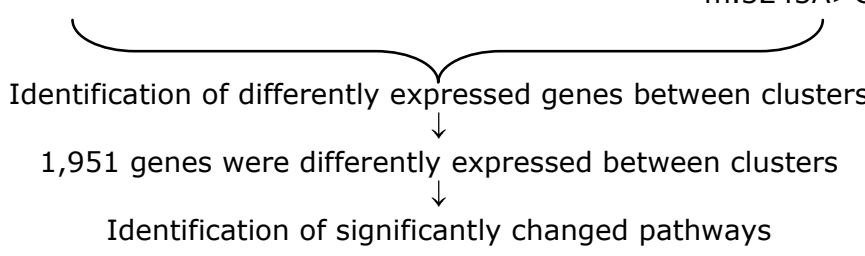

Fig. 1. Microarray analysis procedure

\section{Results}

Subject characteristics and cluster analysis

Skeletal muscle biopsies were obtained from three healthy females that did not carry the m.3243A $>$ G mutation and 8 females with $>10 \%$ of the m.3243A>G mutation in muscle. Characteristics of the m.3243A>G carriers and skeletal muscle at the time of the biopsy are presented in Table 1. Gene expression analysis of skeletal muscle was performed for all $\mathrm{m} .3243 \mathrm{~A}>\mathrm{G}$ carriers and controls (Figure 1). Unsupervised hierarchical clustering was applied on all genes that displayed a larger variation than the median gene variability $(n=13,645)$. The three controls clustered with m.3243A>G patients 33 and 35 , which had a mutation percentage of respectively $76 \%$ and $74 \%$, in cluster 1 . The second cluster comprised six m.3243A $>\mathrm{G}$ patients with a mutation percentage between $27 \%$ and $61 \%$ (Fig. 2). Patient 52 clustered in group 2, but insufficient clinical characteristics were available to determine if she fitted the clustering. The differentially expressed genes and processes, which were responsible for the clusters observed, were characterized in further detail. 


\section{Pathological changes in m.3243A>G carriers}

Correlation
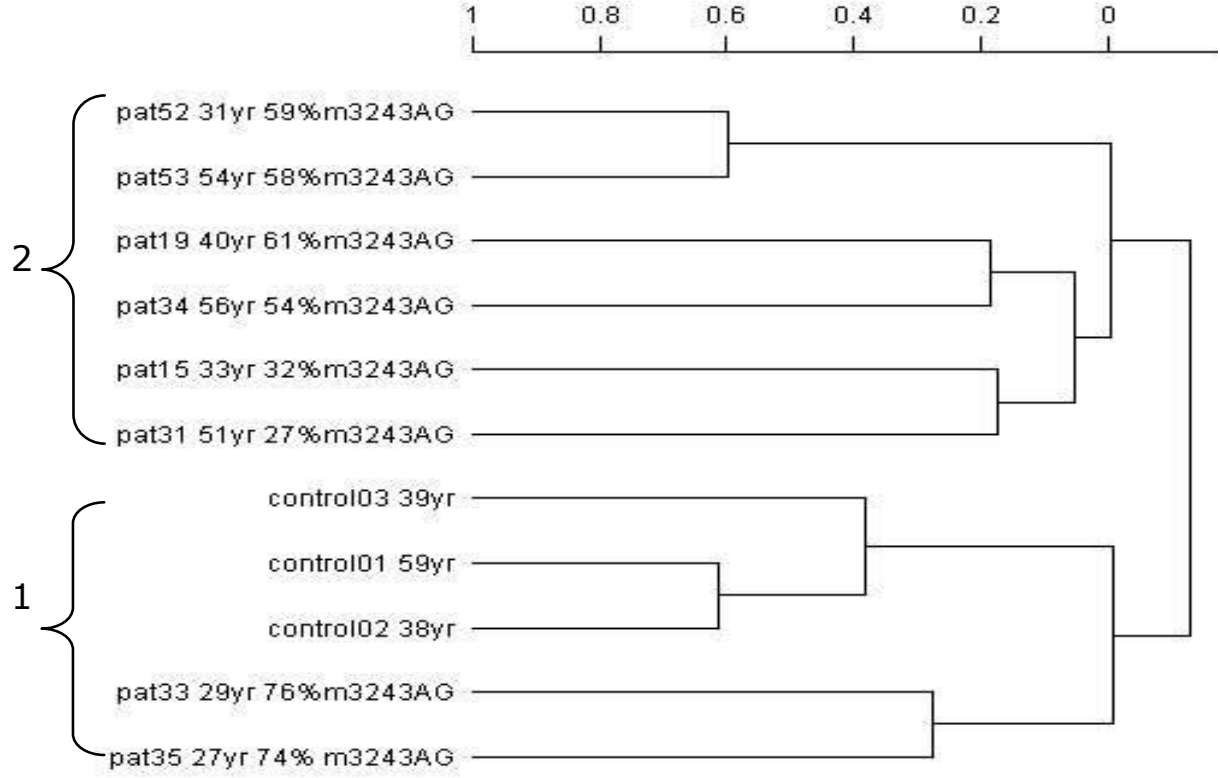

Fig 2. Cluster dendrogram of a hierarchical clustering of 13,645 genes from eight female m.3243A $>$ G carriers and three female controls.

Table 2. qPCR validation of microarray results

\begin{tabular}{lccc}
\hline Gene & Symbol & $\begin{array}{c}\text { Fold change } \\
\text { microarray }( \pm \mathrm{CI})\end{array}$ & $\begin{array}{c}\text { Fold change } \\
\text { qPCR }( \pm \mathrm{CI})\end{array}$ \\
\hline $\begin{array}{l}\text { Protein Kinase, Camp-Dependent } \\
\text { Regulatory, Type II, Beta }\end{array}$ & PRKAR2B & $0.34(0.29-0.40)$ & $0.52(0.28-0.97)$ \\
Actin, Alpha, Cardiac Muscle 1 & ACTC1 & $3.07(2.11-4.45)$ & $4.00(2.16-7.42)$ \\
Cyclin-Dependent Kinase Inhib. & CDKN1A & $2.67(2.27-3.14)$ & $5.05(3.25-7.86)$ \\
Collagen, Type XXI, Alpha 1 & COL21A1 & $1.97(1.39-2.78)$ & $2.11(1.28-3.58)$ \\
Fatty Acid Binding Protein 4 & FABP4 & $0.39(0.32-0.47)$ & $0.60(0.39-0.91)$ \\
Hemoglobin, Beta & HBB & $0.56(0.41-0.77)$ & $0.38(0.15-0.93)$ \\
Phosphofructokinase, Platelet & PFKP & $1.83(1.61-2.07)$ & $1.72(1.12-2.67)$ \\
SH3 And Cysteine Rich Domain 3 & STAC3 & $1.70(1.40-2.07)$ & $1.34(1.13-1.58)$ \\
Mediator Complex Subunit 21 & MED21 & $0.53(0.48-0.77)$ & $0.70(0.55-0.88)$ \\
Alcohol Dehydrogenase 1B, Beta & ADH1B & $0.20(0.16-0.26)$ & $0.55 \mathrm{NS}(0.26-1.12)$ \\
Connective Tissue Growth Factor & CTGF & $1.99(1.19-3.32)$ & $1.36 \mathrm{NS}(0.67-2.75)$ \\
Nicotinamide N-Methyltransferase & NNMT & $2.64(1.40-5.01)$ & $1.65 \mathrm{NS}(0.89-3.09)$ \\
Polymerase (RNA) II & POLR2J & $0.59(0.46-0.97)$ & $0.91 \mathrm{NS}(0.77-1.07)$ \\
(DNA Directed) Polypeptide J & & & \\
RAS Related Protein 10 & RAB10 & $0.28(2.24-0.32)$ & $0.79 \mathrm{NS}(0.59-1.06)$ \\
WW Domain Binding Protein 2 & WBP2 & $1.72(1.37-2.18)$ & $1.13 \mathrm{NS}(0.86-1.49)$ \\
\hline
\end{tabular}

NS: not significantly changed, confidence interval includes 1 


\section{Chapter 3}

Differentially expressed genes between clusters

Of the 17,215 transcripts analyzed, normal regression modelling identified 1,951 genes as differentially expressed with a fold-change of $>10 \%$ between the clusters (ESM 1, see [19]). A total of 1,093 genes were found increased and 882 genes were found decreased in cluster 2 . Fold-changes were generally small, less than two fold in- or decreased. Only 15 of the differentially expressed genes were more than 2 fold higher and 8 transcripts were more than 2 fold lower. As an independent validation of the microarray analysis, qPCR of a number of differentially expressed genes was performed. As shown in Table 2, all genes analyzed with GPCR showed a fold-change in the same direction as observed in the microarray analysis. Nine out of the fifteen genes analyzed were significantly changed on both platforms, and six genes significantly changed on the microarray displayed a non-significant trend with qPCR analysis.

\section{Pathway analysis of differentially expressed genes}

We used a process-based approach to identify differentially expressed pathways between the two clusters. The GenMAPP/MAPPFinder program identified pathways altered in cluster 2, and provided a z-score and permute $p$-value to assess statistical significance. Significantly altered processes could be divided in metabolic and cellular processes. As shown in Table 2 and ESM 2, metabolic processes comprised Krebs cycle, electron transport chain and fatty acid $\beta$-oxidation, which were all predominantly down-regulated in cluster 2 . Altered cellular processes were implicated in cell proliferation and apoptosis, and were both inhibited and stimulated in cluster 2. Furthermore, DAVID program was used to assess significantly enriched GO biological processes at level 5 . In general, GO-based analysis supported the results obtained with pathway analysis, but additionally identified altered processes involved in protein metabolism and muscle regeneration (table 3 and ESM 3, see [19]). 
Table 3. Identification of significantly changed pathways by MAPPFinder analysis

\begin{tabular}{lccccc}
\hline MAPP Name & $\begin{array}{c}\# \\
\text { Changed }\end{array}$ & $\begin{array}{c}\# \\
\text { Measured }\end{array}$ & $\begin{array}{c}\% \\
\text { Changed }\end{array}$ & $\begin{array}{c}\text { Z } \\
\text { Score }\end{array}$ & $\begin{array}{c}\text { Permute } \\
\text { Metabolic processes }\end{array}$ \\
Hs_Electron_Transport_Chain & 43 & 97 & 44 & 8.35 & 0.00 \\
Hs_Krebs-TCA_Cycle & 9 & 31 & 29 & 2.25 & 0.04 \\
Hs_Fatty_Acid_Beta_Oxidation_Meta & 9 & 32 & 28 & 2.14 & 0.03 \\
Hs_Unsat_Fatty_Acid_ß_Oxidation & 5 & 6 & 83 & 4.74 & 0.00 \\
Hs_Mitoch_fatty_acid_ß_oxidation & 7 & 16 & 44 & 3.28 & 0.00 \\
Hs_Glycogen_Metabolism & 10 & 36 & 28 & 2.22 & 0.05 \\
Physiological processes & & & & & \\
Hs_Striated_muscle_contraction & 15 & 38 & 39 & 4.32 & 0.00 \\
Hs_Ribosomal_Proteins & 18 & 53 & 34 & 3.98 & 0.00 \\
Hs_EGFR1_NetPath_4 & 41 & 171 & 24 & 3.50 & 0.00 \\
Hs_Translation_Factors & 14 & 42 & 33 & 3.42 & 0.00 \\
Hs_G13_Signaling_Pathway & 12 & 36 & 33 & 3.16 & 0.01 \\
Hs_TNF-alpha-NF-kB_NetPath_9 & 39 & 177 & 22 & 2.81 & 0.01 \\
Hs_p38_MAPK_signaling_pathway & 10 & 33 & 30 & 2.53 & 0.02 \\
\hline MAPPs with Z-score >1.96 and & permute P $\leq 0.05$ & were & considered & to be & significantly \\
changed & & & & &
\end{tabular}

Table 4. Significantly altered GO biological processes

\begin{tabular}{ll}
\hline Annotation clusters GO biological process level 5 & $\begin{array}{l}\text { Enrichment } \\
\text { score }\end{array}$ \\
\hline Electron transport chain & 8.16 \\
Protein transport & 6.58 \\
RNA processing & 3.57 \\
muscle development & 3.21 \\
Protein import & 2.57 \\
Angiogenesis & 2.16 \\
Protein metabolism & 1.69 \\
Lipid oxidation & 1.60 \\
Polysaccharide metabolism & 1.53 \\
Protein catabolism & 1.52 \\
Mitochondrial complex I assembly & 1.48 \\
Phosphate metabolism & 1.46 \\
Nucleoside metabolism & 1.35 \\
Fatty acid catabolism & 1.33 \\
\hline
\end{tabular}

Enrichment score $>1.30$ are found significantly changed 


\section{Discussion}

The aim of our study was to explore if gene expression profiling of skeletal muscle could explain part of the clinical heterogeneity and/or muscle pathology of the m.3243A>G mutation carriers. Gene expression profiling combined with unsupervised hierarchical clustering divided patients and controls in 2 groups (Fig. 2). Two patients clustered with the controls (cluster 1 ) and the other patients clustered together (cluster 2).

Reduced aerobic energy generation in cluster 2

Expression of a high percentage (44\%) of genes encoding components of all five electron transport chain complexes and the Krebs cycle were reduced in cluster 2 , including malate dehydrogenase 1 (MDH1), which recycles $\mathrm{NADH} / \mathrm{NAD}^{+}$used for ATP synthesis in the mitochondria. In contrast, a number of genes involved in mitochondrial import and utilization of fatty acids for energy generation were increased. For example, increased expression of cytosolic acyl-CoA synthetase longchain family member 3 (ACSL3), carnitine palmitoyltransferase $1 \mathrm{~b}$ $(C P T 1 b)$ and the alpha subunit of the mitochondrial trifunctional protein $(H A D H A)$, which play a key role in lipid biosynthesis, fatty acid degradation, mitochondrial import and $\beta$-oxidation of fatty acids, suggests increased utilization of fatty acids for energy generation. However, increased mitochondrial import of fatty acids and reduced ATP production capacity may increase acetyl-CoA content in the mitochondria, which results in a higher expression of carnitine $\mathrm{O}$ acetyltransferase (CRAT). CRAT overexpression has been shown to inhibit lipid-induced suppression of glucose oxidation [22], but it also catalyzes the reversible transfer of acyl groups from an acyl-CoA thioester to carnitine and regulates the ratio of acylCoA/CoA in the subcellular compartments. In addition, reduced expression of acyl-CoA dehydrogenases ( $A C A D L, A C A D M, A C A D S$ ), which carry out the first step of fatty acid $\beta$-oxidation and expression, may inhibit further build up of acetyl-CoA in this cluster.

Surprisingly, glycogen synthesis was increased in cluster 2. Possibly, increased availability of free fatty acids inhibits insulin-mediated glucose uptake, which results in hyperinsulinemia to accommodate glucose 
uptake by the cells and additionally increases glycogen synthesis [23, 24]. A glucose tolerance test would be required to test this hypothesis. Another potential mechanism of increased glycogen synthesis is increased anaerobic ATP synthesis in these patients, which triggers glycogen synthesis. This phenomenon is also seen during prolonged exhaustive exercise [25].

Increased cellular stress and apoptosis in cluster 2

In addition to processes related to energy metabolism, (signaling) processes implicated in cell proliferation and muscle regeneration were altered (Table 3,4). In cluster 2 , expression was reduced of a number of genes implicated in cell signaling, cytoskeleton reorganization, proliferation and differentiation, suggesting a reduced potential to induce muscle regeneration. For example, Rho GDP-dissociation inhibitor $\beta(A R H G D I B)$, ras-related $C 3$ botulinum toxin substrate $1(R A C 1)$, and expression of cyclin-dependent kinase inhibitor $1 \mathrm{~A}(C D K N 1 A)$, which inhibit cell-cycle progression, were increased, but also a number of genes implicated in muscle regeneration, like myosin 6 (MYH6), MYH8 and mitogen-activated protein kinase kinase 5 (MAP2K5). Furthermore, cluster 2 also displayed both increased and reduced expression of genes involved in translation, protein transport and protein catabolism (Table 4), which may indicate insufficient compensation compared to asymptomatic carriers, who display a consistent upregulation of these processes [12].

Besides inducing cell proliferation, signaling cascades can also trigger an immune response. Expression of a number of pro-inflammatory genes and processes implicated in immune response, like TNFa-NFkB signaling, p38 MAPK signaling, G13 signaling and ribosomal proteins, were increased in cluster 2. Expression of NFKB1 and its binding factor v-rel reticuloendotheliosis viral oncogene homolog $\mathrm{A}$ (RELA) were increased, while expression of their inhibitor NFKBIB was reduced. Ribosomal proteins are also implicated in cell-proliferation and apoptosis, and a number of pro-apoptotic ribosomal proteins, like L13a (RPL13a), RPL5, $R P L 11$ and $R P L 23$, were increased in cluster 2 . These induce apoptosis presumably by triggering $\mathrm{G} 2 / \mathrm{M}$ phase arrest $[26,27]$. In addition, expression of anti-apoptotic RPL35a and B-cell CLL/lymphoma 2 (BCL2) were reduced. Besides increased pro-inflammatory and pro-apoptotic signaling, increased actin cytoskeleton rearrangements also contribute 


\section{Chapter 3}

to cytochrome $c$ release and apoptosis [28]. Two genes involved in this process, cofilin 1 (CFL1) and CFL2, were increased in cluster 2. Recently, actin cytoskeletal rearrangements were identified in m.3243A>G containing lymphoblasts [29]. Interactions between the actin cytoskeleton and mitochondria are required for mitochondrial morphology, movement, apoptosis, and actin polymerization and, consequently, cytoskeleton changes may also lead to secondary mitochondrial dysfunction in m.3243A $>G$ containing cells. Mitochondrial dysfunction in m.3243A>G carriers is also associated with increased formation of reactive oxygen species and endoplasmatic reticulum stress $[30,31]$, reflected by increased DNA-damage-inducible transcript 3 (DDIT3) expression. Taken together, these data suggests that the patients in cluster 2 have increased cellular stress and expression of genes involved in cell death, which is not accompanied by a significant induction of cell regeneration, and likely results in a reduced number of functional cells.

\section{Cluster 1 with the controls and 2 patients}

A striking observation was that 2 of the affected patients (33 and 35) clustered with the controls in cluster 1 . These patients were the youngest and had the highest mutation load. Both suffered from diabetes, but neuromuscular manifestations were mild. Patients in cluster 2 were more heterogeneous, but were either older or more severely affected and only one developed diabetes, yet at an older age. Our data are consistent with the reported correlation between age at onset of diabetes and skeletal muscle mutation load [4]. The high percentage in muscle reflects an overall high mutation load in all tissues, predisposing to neuromuscular pathology later in life. As no clear gene expression differences were observed in muscle between the controls and patients 33 and 35, this may indicate that diabetes does not primarily result from muscle alterations, but more likely from pancreatic $\beta$-cell dysfunction $[24,32]$. In contrast to common T2DM patients, mitochondrial diabetics quickly progress towards exogenous insulindependence because of progressive $\beta$-cell failure. Nearly all m.3243A>G carriers develop diabetes during life and ageing, which is associated with a gradual decline in muscle mass. Therefore, muscle alterations are clearly involved as an additional contributing factor. Development of additional pathology is not essential as diabetes can be the sole clinical manifestation, but it may still contribute to the development of diabetes, 
especially in the lower mutation range. Studying pancreatic $\beta$-cells and skeletal muscle of mitochondrial diabetes patients will increase our understanding of insulin resistance and mitochondrial malfunctioning in common T2DM patients. Since mitochondrial diabetes patients are in general not obese, there is no obesity effect nor altered adipokine signaling present.

The patients in cluster 1 also differ in severity with the young patients $(15,19$ and 52$)$ or in age with the more mildly affected patients $(31,34$ and 53) of cluster 2 . Gene expression differences between cluster 1 and 2 suggest that disease progression and aging trigger a common gene expression profile of reduced mitochondrial function and reduced muscle regeneration. The inability to distinguish between young severely affected and older mildly affected individuals of cluster 2 indicates that other genetic or environmental factors, implicated in disease severity and progression, remain to be identified. This is in agreement with previous studies which observed that myopathy is a common feature of m.3243A > G carriers with large variability between patients irrespective of clinical phenotype $[6,32]$. In addition, maximum oxygen uptake and mitochondrial energy capacity system (MEGS) capacity in muscle have been found to correlate with the m.3243A>G mutation percentage with a clear involvement of aging, but this did not correlate with the clinical presentation [11,33]. Furthermore, the m.3243A>G mutation causes an increase in the production of reactive oxygen species [12]. Reactive oxygen species are functional, for example for muscle regeneration, but are highly detrimental when they are in excess. Exploring the balance between ROS formation and clearance in m.3243A>G carriers in different tissues with different ROS sensitivities would be a relevant factor in m.3243A>G related pathologies. Alternatively, myopathy, exercise intolerance and muscle weakness can also be secondary effects of severely affected m.3243A>G carriers who have adopted a more sedentary life-style due to high disease burden of other tissues. Exercise training has been shown to be an effective intervention to increase muscle mass and mitochondrial function in elderly [34], T2DM patients [35], but also in carriers of a mitochondrial DNA mutation including m.3243A $>G$ carriers [36]. Therefore, exercise or pharmacological compounds aimed at stimulating muscle regeneration and mitochondrial biogenesis in m.3243A $>\mathrm{G}$ carriers may delay onset and/or reduce severity of symptoms in these patients. However, therapies should be 


\section{Chapter 3}

carefully controlled to prevent increased lactate production or enhance formation of reactive oxygen species in m.3243A>G carriers.

In summary, our unsupervised clustering of gene expression data resulted in two clusters of patients. An earlier study showed that asymptomatic carriers have increased expression of OXPHOS subunits [12] compared to healthy controls. The picture for the symptomatic patients is less straightforward, although our data reveal intriguing differences in the underlying pathological processes. Combining gene expression data with data on muscle strength, physical activity, maximal oxygen uptake, MEGS capacity and oxidative stress will likely improve our understanding of these differences and their relation to the clinical phenotype compared with the skeletal muscle parameters analyzed so far. The same would be true for other tissues, although the accessibility of these tissues in patients might be a key problem.

\section{Acknowledgements}

The research presented was funded by the Dutch Diabetes Foundation (DFN 2004.00.040). 


\section{References}

1. Majamaa, K., et al., Epidemiology of A3243G, the mutation for mitochondrial encephalomyopathy, lactic acidosis, and strokelike episodes: prevalence of the mutation in an adult population. Am J Hum Genet, 1998. 63(2): p. 447-54.

2. Chomyn, A., et al., The mitochondrial myopathy, encephalopathy, lactic acidosis, and stroke-like episode syndrome-associated human mitochondrial tRNALeu(UUR) mutation causes aminoacylation deficiency and concomitant reduced association of mRNA with ribosomes. J Biol Chem, 2000. 275(25): p. 19198-209.

3. Park, H., E. Davidson, and M.P. King, The pathogenic A3243G mutation in human mitochondrial tRNALeu(UUR) decreases the efficiency of aminoacylation. Biochemistry, 2003. 42(4): p. 958-64.

4. Maassen, J.A., et al., Mitochondrial diabetes: molecular mechanisms and clinical presentation. Diabetes, 2004. 53 Suppl 1: p. S103-9.

5. Chinnery, P.F., et al., Molecular pathology of MELAS and MERRF. The relationship between mutation load and clinical phenotypes. Brain, 1997. 120 ( Pt 10): p. 1713-21.

6. Karppa, M., et al., Spectrum of myopathic findings in 50 patients with the 3243A>G mutation in mitochondrial DNA. Brain, 2005. 128(Pt 8): p. 1861-9.

7. Tanaka, M., et al., Mitochondrial DNA mutations in mitochondrial myopathy, encephalopathy, lactic acidosis, and stroke-like episodes (MELAS). Biochem Biophys Res Commun, 1991. 174(2): p. 861-8.

8. Pavlakis, S.G., et al., Mitochondrial myopathy, encephalopathy, lactic acidosis, and strokelike episodes: a distinctive clinical syndrome. Ann Neurol, 1984. 16(4): p. 481-8.

9. Pierron, D., et al., New evidence of a mitochondrial genetic background paradox: impact of the $\mathrm{J}$ haplogroup on the A3243G mutation. BMC Med Genet, 2008. 9: p. 41.

10. Tatuch, Y., et al., The 8993 mtDNA mutation: heteroplasmy and clinical presentation in three families. Eur J Hum Genet, 1994. 2(1): p. 35-43.

11. Jeppesen, T.D., et al., Muscle phenotype and mutation load in 51 persons with the 3243A>G mitochondrial DNA mutation. Arch Neurol, 2006. 63(12): p. 17016.

12. van Eijsden, R.G., et al., Termination of damaged protein repair defines the occurrence of symptoms in carriers of the m.3243A > G tRNA(Leu) mutation. J Med Genet, 2008. 45(8): p. 525-34.

13. http://brainarray.mbni.med.umich.edu. [cited; chip description file]. Available from: http://brainarray.mbni.med.umich.edu.

14. Dai, M., et al., Evolving gene/transcript definitions significantly alter the interpretation of GeneChip data. Nucleic Acids Res, 2005. 33(20): p. e175.

15. Akaike, H. Information theory and an extension of the maximum likelihood principle. in Second International Symposium on Inference Theory. 1973. Budapest: Akadémiai Kiadó.

16. Salomonis, N., et al., GenMAPP 2: new features and resources for pathway analysis. BMC Bioinformatics, 2007. 8: p. 217.

17. Doniger, S.W., et al., MAPPFinder: using Gene Ontology and GenMAPP to create a global gene-expression profile from microarray data. Genome Biol, 2003. 4(1): p. R7.

18. Dennis, G., Jr., et al., DAVID: Database for Annotation, Visualization, and Integrated Discovery. Genome Biol, 2003. 4(5): p. P3.

19. Van Tienen, F.H. Supplementary material thesis. 2010 [cited; Available from: http://www.personeel.unimaas.nl/florence-vantienen/.

20. Ihaka R, G., R "R: a language for data analysis and graphics". Journal of Computational Graphics and Statistics, 1996. 5(3): p. 299-314.

21. Lindsey, J., Models for repeated measurements, 2nd edition. 1999, Oxford: Oxford University Press. 536. 
22. Noland, R.C., et al., Carnitine insufficiency caused by aging and overnutrition compromises mitochondrial performance and metabolic control. J Biol Chem, 2009. 284(34): p. 22840-52.

23. Morino, K., et al., Reduced mitochondrial density and increased IRS-1 serine phosphorylation in muscle of insulin-resistant offspring of type 2 diabetic parents. J Clin Invest, 2005. 115(12): p. 3587-93.

24. Lindroos, M.M., et al., The m.3243A>G mutation in mitochondrial DNA leads to decreased insulin sensitivity in skeletal muscle and to progressive \{beta\}-cell dysfunction. Diabetes, 2008.

25. Shearer, J., et al., Increases in glycogenin and glycogenin mRNA accompany glycogen resynthesis in human skeletal muscle. Am J Physiol Endocrinol Metab, 2005. 289(3): p. E508-14.

26. Chen, F.W. and Y.A. Ioannou, Ribosomal proteins in cell proliferation and apoptosis. Int Rev Immunol, 1999. 18(5-6): p. 429-48.

27. Lindstrom, M.S., Emerging functions of ribosomal proteins in gene-specific transcription and translation. Biochem Biophys Res Commun, 2009. 379(2): p. 167-70.

28. Klamt, F., et al., Oxidant-induced apoptosis is mediated by oxidation of the actinregulatory protein cofilin. Nat Cell Biol, 2009. 11(10): p. 1241-6.

29. Felk, S., et al., Activation of the mitochondrial protein quality control system and actin cytoskeletal alterations in cells harbouring the MELAS mitochondrial DNA mutation. J Neurol Sci.

30. Woo, C.W., et al., Adaptive suppression of the ATF4-CHOP branch of the unfolded protein response by toll-like receptor signalling. Nat Cell Biol, 2009. 11(12): p. 1473-80.

31. Cortopassi, G., et al., Mitochondrial disease activates transcripts of the unfolded protein response and cell cycle and inhibits vesicular secretion and oligodendrocyte-specific transcripts. Mitochondrion, 2006. 6(4): p. 161-75.

32. Frederiksen, A.L., et al., High prevalence of impaired glucose homeostasis and myopathy in asymptomatic and oligosymptomatic 3243A>G mitochondrial DNA mutation-positive subjects. J Clin Endocrinol Metab, 2009. 94(8): p. 2872-9.

33. Janssen, A.J., et al., Muscle 3243A-->G mutation load and capacity of the mitochondrial energy-generating system. Ann Neurol, 2008. 63(4): p. 473-81.

34. Verdijk, L.B., et al., Skeletal muscle hypertrophy following resistance training is accompanied by a fiber type-specific increase in satellite cell content in elderly men. J Gerontol A Biol Sci Med Sci, 2009. 64(3): p. 332-9.

35. Bordenave, S., et al., Training-induced improvement in lipid oxidation in type 2 diabetes mellitus is related to alterations in muscle mitochondrial activity. Effect of endurance training in type 2 diabetes. Diabetes Metab, 2008. 34(2): p. 162-8.

36. Taivassalo, T., et al., The spectrum of exercise tolerance in mitochondrial myopathies: a study of 40 patients. Brain, 2003. 126(Pt 2): p. 413-23. 


\section{Chapter 4}

\section{Preadipocytes of Type 2 Diabetes subjects display an intrinsic gene expression profile of decreased differentiation capacity}

F.H.J. van Tienen, C.J.H. van der Kallen, P.J. Lindsey,
R.J. Wanders, M.M. van Greevenbroek,
H.J.M. Smeets 


\section{Chapter 4}

\section{Abstract}

Insulin resistance and Type 2 Diabetes Mellitus (T2DM) are associated with increased adipocyte size, altered secretory pattern and decreased differentiation of preadipocytes. In this study, we identify the underlying molecular processes in preadipocytes of T2DM patients that are a characteristic of the development of T2DM. Preadipocyte cell cultures were prepared from subcutaneous fat biopsies of 7 T2DM patients (age

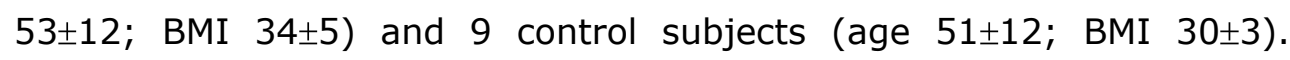
Microarray analysis was used to identify altered processes between the T2DM and control preadipocytes. Gene expression profiling showed changed expression of transcription regulators involved in adipogenesis and in extracellular matrix remodeling, actin cytoskeleton and integrin signaling genes, which indicates decreased capacity to differentiate. Additionally, genes involved in insulin signaling and lipid metabolism were down-regulated, and inflammation/apoptosis was up-regulated in T2DM preadipocytes. Decreased expression of genes involved in differentiation can provide a molecular basis for the reduced adipogenesis of preadipocytes of T2DM subjects, leading to reduced formation of adipocytes in subcutaneous fat depots, and ultimately leading to ectopic fat storage.

\section{Introduction}

Type 2 diabetes mellitus (T2DM) is characterized by hyperglycemia, impaired insulin secretion from pancreatic $\beta$-cells and insulin resistance in peripheral tissues, including metabolically important tissues like liver, skeletal muscle and adipose tissue [1]. Over the past ten years, the incidence of T2DM has increased dramatically with an expected rise to about 300 million patients world wide in 2025 [2, 3]. The development of T2DM is generally preceded by obesity, as $80 \%$ of the patients are obese (BMI $>30 \mathrm{~kg} / \mathrm{m}^{2}$ ), which also increases the risk of other obesityassociated diseases, like cardiovascular diseases [4, 5]. Adipose tissue was once thought to be an inert energy storage depot, but is now known as a critical endocrine organ. It secretes a range of peptides, hormones and other signaling molecules that play a role in a large number of diverse processes, like glucose and lipid homeostasis [6-8]. Changes in 
the secretory pattern of adipocytes are observed in obesity, T2DM and cardiovascular diseases [9].

Adipose tissue is composed of various subcutaneous and visceral depots throughout the body, and is composed of mature energy storing adipocytes, stromal vascular cells (SVCs) and adipocyte precursor cells called preadipocytes [10]. Enlarged adipocytes are found in patients with T2DM and pre-diabetic individuals and appear to be an independent marker of insulin resistance in pre-diabetic subcutaneous adipose tissue [11]. Gene expression analysis of adipocytes and adipose tissue of patients with insulin resistance has revealed a reduced expression of adipose tissue specific proteins and of adipogenic transcription regulators, like peroxisome proliferator-activated receptor $\mathrm{Y}(P P A R Y)$, CCAAT/ehancer-binding proteins (C/EBPS) and sterol regulatory elements binding proteins (SREBPS) [12]. In addition, adipose tissue inflammation in obese and insulin resistant individuals impairs the differentiation of preadipocytes and reduces the oxidative capacity of visceral adipose tissue [13-15].

So far, most gene expression studies focus on the role of adipocytes in the development of T2DM and preadipocyte gene expression analysis were mainly used to study obesity $[12,13,16]$. Our study is the first to perform global gene expression profiling of preadipocytes of T2DM patients. We aim to identify processes in preadipocytes of T2DM patients and non-diabetic age- and BMI-matched controls that are a characteristic of T2DM.

\section{Materials \& Methods}

\section{Subjects}

Our study subjects were 7 patients with T2DM and 9 normoglycemic controls within the same age and BMI range as the T2DM subjects (Table 1). Lipid-lowering medication was stopped two weeks before biopsy, 3 of 7 T2DM subjects continued insulin treatment, one T2DM subject continued with metformin treatment and three T2DM subjects did not use glucose lowering medication. T2DM and controls were recruited through the Lipid Clinic of the Maastricht University Medical 


\section{Chapter 4}

Center (Maastricht UMC). The Human Investigation Review Committee of the MUMC approved the study protocol and all subjects gave written informed consent.

Table 1. Characteristics of control and T2DM subjects

\begin{tabular}{llll}
\hline Characteristic & Control group & T2DM group & $p$ value \\
\hline Nr. of subjects (male/female) & $4 / 5$ & $2 / 5$ & \\
Age $($ years $)$ & $51 \pm 12$ & $53 \pm 12$ & $>0.05$ \\
BMI $\left(\mathrm{kg} / \mathrm{m}^{2}\right)$ & $30 \pm 3$ & $34 \pm 5$ & $>0.05$ \\
Insulin $(\mu \mathrm{U} / \mathrm{mL}) * \#$ & $7.81 \pm 2.92$ & $20.25 \pm 9.87$ & 0.01 \\
Glucose $(\mathrm{mmol} / \mathrm{L}) *$ & $5.26 \pm 0.43$ & $10.72 \pm 2.57$ & 0.001 \\
Waist-hip ratio & $0.97 \pm 0.01$ & $0.97 \pm 0.09$ & $>0.05$ \\
Cholesterol $(\mathrm{mmol} / \mathrm{L}) *$ & $4.33 \pm 0.93$ & $5.74 \pm 0.60$ & 0.01 \\
Triglycerides $(\mathrm{mmol} / \mathrm{L}) * *$ & $1.10 \pm 0.25$ & $3.68 \pm 1.51$ & $<0.001$ \\
\hline
\end{tabular}

Data are means \pm SD.

* Characteristic with $\mathrm{p}$-value $<0.05$ using student T-test

\# log transformed before analysis

\section{Preadipocyte isolation and culture}

At fasted state, subcutaneous adipose tissue biopsy samples were taken caudally from the umbilicus by liposuction under local anesthesia and subsequently treated with collagenase [17]. The cell suspension was filtered through $500 \mu \mathrm{m}$ nylon mesh and spun at $220 \times g$ for $1 \mathrm{~min}$ to separate preadipocytes from mature adipocytes. Preadipocytes were suspended in $5 \mathrm{ml}$ DMEM medium containing $10 \%$ fetal calf serum (Gibco-Invitrogen, Carlsbad, USA) and 1\% glutamax/ penecillin/streptomycin (Gibco-Invitrogen), grown for 2 passages and frozen. Control and T2DM preadipocytes were taken into culture in parallel and cultured for 3 passages till a $90 \%$ confluent $162 \mathrm{~cm}^{2}$ culture flask was obtained per sample (passage 5 - 9) for RNA isolation.

\section{Microarray procedure}

Total RNA was isolated using the TRIzol reagent (Invitrogen, Carlsbad, USA) and purified with the RNeasy clean-up kit (Qiagen, Valencia, USA), according to manufacturers' protocol. RNA quantity and purity were determined spectrophotometrically using the Nanodrop ND-1000 (Nanodrop Technologies, Wilmington, USA). $1 \mu \mathrm{g}$ RNA from each sample with a RNA integrity number (RIN) >7 (Bioanalyser 2100; Agilent, Santa Clara, USA), was amplified using the Amino-Allyl MessageAmp II aRNA 
kit (Ambion, Austin, USA) to generate amino-allyl aRNA (AA-aRNA). AAaRNA was generated according to manufacturer's protocol with a modification in the ratio UTP: AA-UTP, which was changed from 1:1 to 7:3. RNA integrity was assessed by determining the aRNA size. Each amino AA-aRNA sample was coupled to Cy3 or Cy5 (Amersham Biosciences, GE healthcare, Little Chalfont, UK) and purified with the RNeasy Minelute clean-up kit (Qiagen). RNA quantity and dye incorporation were determined spectrophotometrically using the Nanodrop. Hybridization was performed using the Operon Human oligo collection V2 (21 521 features) spotted on CodeLink slides (Amersham Biosciences) printed by the Microarray Facility (University Medical Center Utrecht, The Netherlands). T2DM patients and controls were paired on each slide based on respectively sex, age and BMI. Two-color microarray hybridizations were performed including dye-swap for technical replication. Hybridizations, washings, and slide drying were performed on a Tecan HS4800 hybridization station. Slides were blocked in 5xSSC, $25 \%$ Formamide, $0.1 \%$ SDS, 1\% BSA (Sigma, St Louis, USA) for 1 hour. $120 \mathrm{pmol}$ of each dye-labeled aRNA sample (2\% - $4.5 \%$ dye incorporation) was fragmented using RNA fragmentation buffer (Ambion), mixed with $100 \mu \mathrm{g}$ tRNA (Roche, Mannheim, Germany) and $50 \mu \mathrm{g}$ Herring sperm DNA (Invitrogen) in $120 \mu$ hybridization solution [5xSSC, 25\% Formamide, $0.1 \%$ SDS], denaturated for 5 minutes at $95^{\circ} \mathrm{C}$, hybridized for 16 hours at $42^{\circ} \mathrm{C}$ and washed 3 times with decreasing concentrations of SSC and SDS (1xSSC, $0.2 \%$ SDS; $0.1 \times S S C$, $0.2 \%$ SDS; $0.1 \times S S C)$. Array scanning was performed on an Axon 4000B scanner with laser power $100 \%$ for both channels and three photo multiplier settings for respectively the red and the green channel (1000 and 888 to detect low expressed probes, 756 and 671 to detect highly expressed probes ( $\leq 0.05 \%$ of all spots saturated) and an intermediate setting of 878 and 779). Scanned images were analyzed using Array-Pro analyzer software (MediaCybernetics, Bethesda, USA). All microarray data is deposited in the GEO database, accession GSE15790.

Microarray data statistical analysis

First, each gene was analyzed using a regression model [18]. The Akaike Information Criterion (AIC) was used to identify the differently expressed genes. Then, these are mapped to processes, which are ranked by their $z$-scores and permute $p$-values. Bad and missing spots were not included in the analysis. Secondly, the scanning power with the 


\section{Chapter 4}

least missing and most values above background for each gene were kept. If more than one scan setting was suitable for a spot, then the scanning at medium power was preferred over high power, which was itself preferred over the low setting. Scanning settings for each gene was constant between arrays.

A more detailed description of this analysis is shown in the supplementary data (ESM 5). All ESM can be found at http://www.personeel.unimaas.nl/florence-vantienen/, also indicated by reference [19]. Briefly, the foreground intensities of the remaining genes were analyzed using a multivariate Gaussian linear regression including labeling efficiency, dye swap, hybridization days, background level, sex, age, BMI and a random effect to take into account multiple observations on the same subject. The inference criterion used for comparing the models is their ability to predict the observed data, i.e. models are compared directly through their minimized minus log-likelihood. When the numbers of parameters in models differ, they are penalized by adding the number of estimated parameters, a form of the AIC [20]. For each gene, the group was then added to the model. The gene under consideration was found to be differentially expressed if the AIC was smaller when compared to the AIC of the model not containing the group effect.

The genes and their fold changes were loaded into the Gene Map Annotator (GenMAPP, version 2.0) [21] and Pathway Profiler (MAPPFinder) [22] to evaluate the transcripts in relation to known biological processes, molecular function and cellular component based on Gene Ontology (GO) terms [23] and local maps. Gene database Hsstd_20060526 and MAPPs version Hs_contributed_20070308 were used to obtain a ranked list of pathways with differentially expressed genes. MappFinder software was used to select the MAPPs with relatively high numbers of differentially expressed genes. The ranking of regulated pathways was indicated by the individual z-scores. The z-score increased when higher numbers of changing genes were found, taking into account the number of genes present in the MAPP that were represented on the array, and the total number of genes involved in the concerning MAPP. MAPPs were selected for further study if the z-score was greater than 1.96 . 
Real-time quantitative $P C R$ validation

Differentially expressed genes were validated by real-time quantitative PCR ( $q P C R$ ) on the same RNA samples, as used for the microarrays. Primers were designed using Primer Express $\AA$ software version 3.0 (Applied Biosystems), (sequences primers, see ESM Table 4 [19]). cDNA was synthesized using Superscript II cDNA synthesis kit (Invitrogen). Quantification was carried out using BioRad MyiQ Single-Color Real-Time PCR detection system using qPCR Mastermix Plus for SYBR Green $®$ I (Eurogentec, Seraing, B). The cycling conditions were: an initial step for $2^{\prime}$ at $50^{\circ} \mathrm{C}$, activation of the enzyme at $95^{\circ} \mathrm{C}$ for $10^{\prime}$, and 40 cycles of $15^{\prime \prime}$ at $95^{\circ} \mathrm{C}$ followed by $1^{\prime}$ at $60^{\circ} \mathrm{C}$. The mRNA levels of each gene were normalized to those of the housekeeping gene encoding TATA-box binding protein (TBP), which was invariable between the preadipocyte samples. For best comparison, results were analyzed using Gaussian linear regression, similar to the microarray analysis. The housekeeping gene, age, sex, and BMI were included in the analysis. The AIC was used to assess whether there was a difference between the controls and T2DM subjects (group effect). All statistical analysis presented were performed using the freely available program $R$ [24] and the publicly available library 'growth' [25].

\section{CPT1 activity assay}

CPT1 activity was measured in preadipocytes by tandem mass spectrometry as published by van Vlies et al. [26] except that digitonin was used in a concentration of $20 \mu \mathrm{g} / \mathrm{ml}$ at a final protein concentration of $0.3 \mathrm{mg} / \mathrm{ml}$.

\section{Preadipocyte differentiation}

The human preadipocyte cell-line from a patient with Simpson-GolabiBehmel Syndrome (SGBS), which has a high capacity for adipose differentiation, was used to explore the CPT1a and CPT1b expression pattern during preadipocyte differentiation. SGBS preadipocytes were differentiated according to the protocol as published by Wabitsch et al. [27]. 


\section{Chapter 4}

\section{Results}

Validated microarray gene expression data

Microarray analysis was performed on RNA from preadipocyte cultures of 7 T2DM and 9 control subjects (Table 1), comparable in age, BMI and waist-to-hip ratio. T2DM subjects had elevated insulin and glucose levels, whereas control subjects were normoglycemic. Cholesterol and triglyceride levels were higher in the T2DM subjects. Purity of the culture and infiltration of other cell-types like fibroblasts was checked under a light microscope and by monitoring expression of macrophage, endothelial cell, and stem cell specific genes. No differences were found between the T2DM and control group for endothelial cell or stem cell specific genes, and only 1 of 46 macrophage specific genes was differentially expressed, namely cytochrome b-245 (ESM Table 2 [19]).

To assess the consistency of the microarrays, 12 probes were spotted 17 times on each array and analysed individually. Eleven of the 12 probes gave a signal above detection level with a consistent fold change. All replicates of three probes were decreased (fold change $<0.90$ ), of one probe increased (fold change $>1.10$ ), and expression of the replicates from the other 7 probes were unchanged between T2DM patients and controls. The decreased expression of one of the probes spotted multiple times, namely GAPDH, was also verified by qPCR. Expression data from both platforms were analyzed in a similar manner (Fig. 2). Twenty significantly altered genes on the microarray were changed in the same direction using qPCR, which was significant for 13 genes. The seven remaining genes were significantly changed on the microarray, but had a confidence interval including one with GPCR analysis although their means pointed towards changes in the same direction. The correlation of the 20 genes analyzed on both platforms was 0.82 , with $p<0.01$ (data not shown).

Genes differentially expressed in T2DM preadipocytes

Using two-color microarrays, 21,337 transcripts were analyzed, and 8,678 of those had signal intensity higher than twice the background intensity. Normal linear regression modeling indicated that 1,144 transcripts were differentially expressed with a fold change of minimal $10 \%$, of which, 431 transcripts were increased, and 713 transcripts were decreased in the T2DM preadipocytes (ESM Table 1 and Fig. 1 [19]). 
Fold changes were in general small, 39 of the 713 decreased transcripts $(5.5 \%)$ had a fold change $<0.5$, and the most extreme reduced probe had a fold change of 0.29 . Of the 431 increased transcripts in the T2DM group, only 8 transcripts $(1.9 \%)$ were more than two times higher with a maximum of 2.98 .

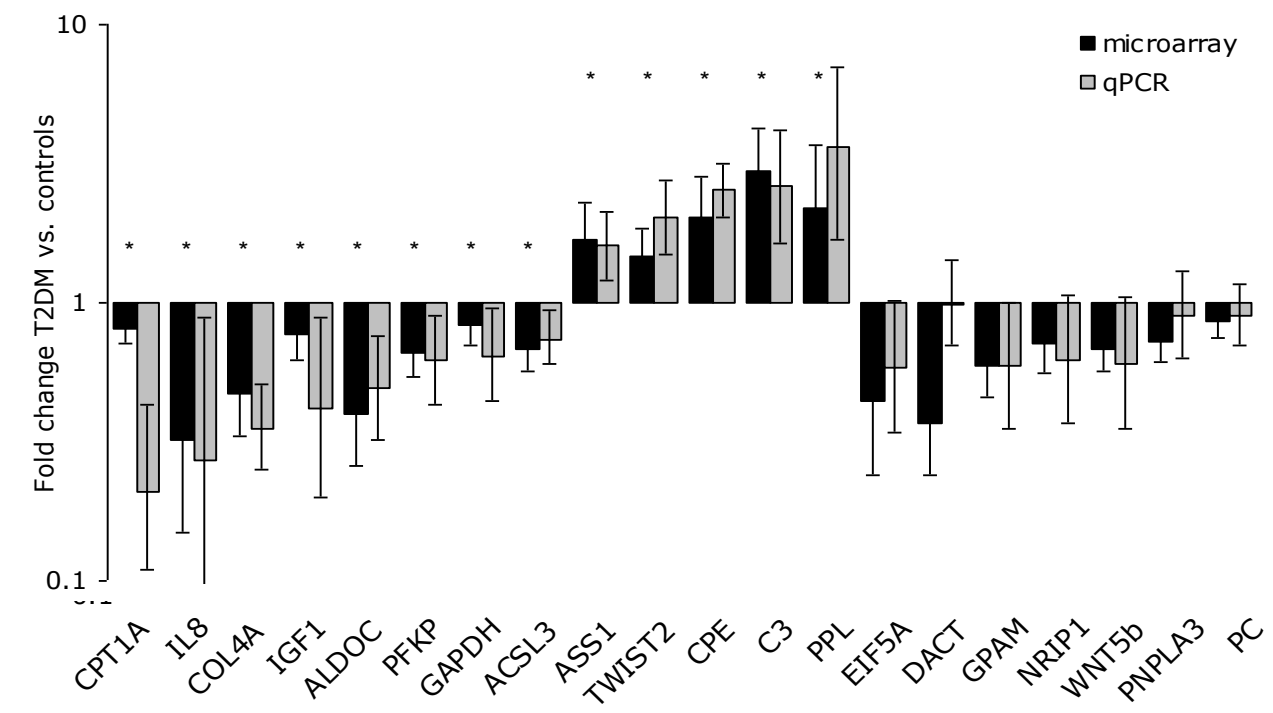

Fig. 1. Validation of microarray analysis by qPCR

Expression of 20 genes that were significantly changed in the microarray analysis were analyzed by qPCR. The fold-change of the T2DM preadipocytes \pm CI of each gene on both platforms is shown. * Genes that are significantly changed on both platforms.

Process-based analysis of differentially expressed genes

We used a process-based approach to identify differentially expressed pathways between preadipocytes from T2DM and control subjects. The 886 differently expressed genes that could be annotated were used for gene ontology based analysis using the GenMAPP/MAPPFinder program (ESM Table 3 [19]). Thirteen pathways (Table 2) were found significantly changed with MAPPFinder analysis with a z-score $>1.96$, and permute $p$-value $<0.10$. The most significantly changed local pathways or gene groups comprised adipogenesis, alanine and aspartate metabolism, glycerolipid metabolism, insulin signaling, ribosomal proteins, androgen receptor signaling and focal cell adhesion.

In addition, $38 \mathrm{GO}$ biological process terms were significantly changed with a z-score $>1.96$ and a minimum of 3 changed genes per term (Table 3). Detailed information about the function of differently 
expressed genes was obtained using Entrez Gene, OMIM and Pubmed to complete the altered processes represented in GenMAPP.

Table 2. Identification of significantly changed pathways by MAPPFinder analysis

\begin{tabular}{|c|c|c|c|c|c|}
\hline MAPP Name & $\begin{array}{l}\text { Changed } \\
\text { (n) }\end{array}$ & $\begin{array}{l}\text { Measured } \\
\quad(n)\end{array}$ & $\begin{array}{c}\text { Changed } \\
(\%)\end{array}$ & Z Score & $\begin{array}{c}\text { Permute } \\
P\end{array}$ \\
\hline \multicolumn{6}{|l|}{ Adipogenesis } \\
\hline Hs_Adipogenesis & 17 & 108 & 15.74 & 3.22 & 0.001 \\
\hline Hs_Focal_adhesion_KEGG & 22 & 149 & 14.77 & 3.34 & 0.003 \\
\hline Hs_MAPK_signaling_pathway & 16 & 130 & 12.31 & 2.05 & 0.029 \\
\hline Hs_Insulin_Signaling & 20 & 136 & 14.71 & 3.16 & 0.005 \\
\hline \multicolumn{6}{|l|}{ Metabolism } \\
\hline Hs_Glycerolipid_metabolism & 10 & 69 & 14.49 & 2.17 & 0.036 \\
\hline \multicolumn{6}{|l|}{ Inflammation / Apoptosis } \\
\hline $\begin{array}{l}\text { Hs_Complement and Coagulation } \\
\text { Cascades_KEGG }\end{array}$ & 7 & 44 & 15.91 & 2.09 & 0.057 \\
\hline \multicolumn{6}{|l|}{ Unclassified } \\
\hline Hs_Ribosomal_Proteins & 10 & 61 & 16.39 & 2.61 & 0.019 \\
\hline Hs_Androgen-Receptor_NetPath_2 & 18 & 91 & 19.78 & 4.42 & 0.000 \\
\hline Hs_IL-7_NetPath_19 & 8 & 33 & 24.24 & 3.62 & 0.003 \\
\hline Hs_Id_NetPath_5 & 7 & 40 & 17.50 & 2.37 & 0.018 \\
\hline Hs_IL-9_NetPath_20 & 4 & 19 & 21.05 & 2.22 & 0.049 \\
\hline Hs_Prostaglandin_synth_regulation & 5 & 29 & 17.24 & 1.97 & 0.074 \\
\hline
\end{tabular}

z-score values $>1.96, p<0.10$, and number changed $>2$ were considered to be significant.
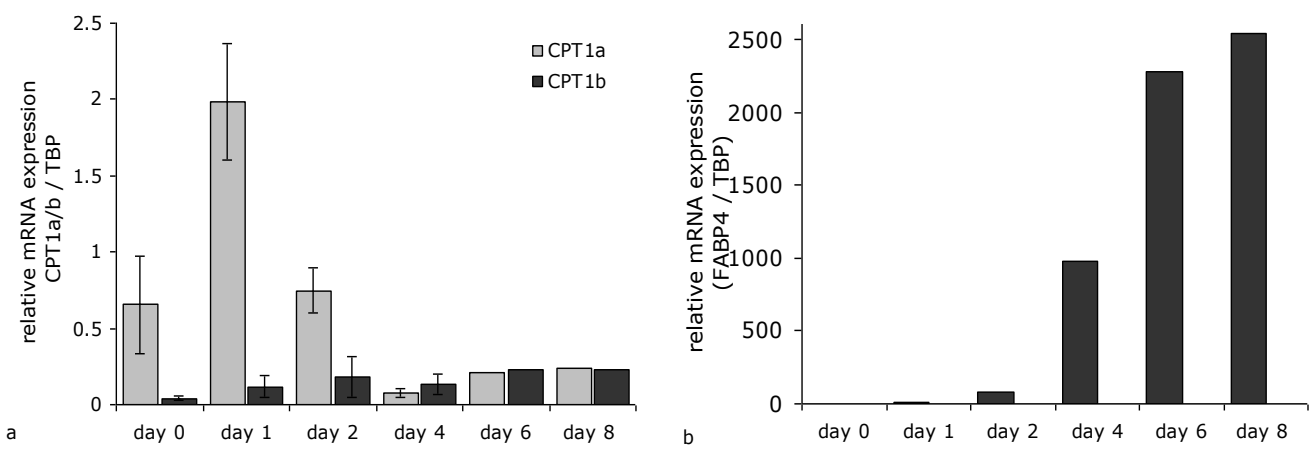

Fig. 2. Expression pattern of $C P T 1 a, C P T 1 b$ and $F A B P 4$ during differentiation of SGBS preadipocyte cell-line. (a) Relative expression of CPT1a and CPT1b. (b) FABP4 expression as marker for differentiation. Total RNA was obtained at different days after induction of differentiation and subjected to GPCR. The expression was normalized with TBP expression. The bars indicate S.E. $(n=2)$. 


\section{Intrinsic gene expression changes in T2DM preadipocytes}

Table 3. Identification of significantly changed GO terms biological processes by MAPPFinder

\begin{tabular}{|c|c|c|c|c|c|c|}
\hline $\begin{array}{l}\text { GO } \\
\text { ID }\end{array}$ & GO term & $\begin{array}{l}\text { Changed } \\
(\mathrm{n})\end{array}$ & $\begin{array}{l}\text { Measured } \\
\text { (n) }\end{array}$ & $\begin{array}{c}\text { Changed } \\
(\%)\end{array}$ & $\begin{array}{c}\text { Z } \\
\text { Score }\end{array}$ & Permute $\mathrm{P}$ \\
\hline 7265 & Ras protein signal transduc & 7 & 18 & 39 & 5.25 & 0.000 \\
\hline 50792 & regulation of viral life cycle & 3 & 6 & 50 & 4.09 & 0.004 \\
\hline 6289 & nucleotide-excision repair & 3 & 6 & 50 & 4.09 & 0.010 \\
\hline 7016 & cytoskeletal anchoring & 3 & 7 & 43 & 3.68 & 0.007 \\
\hline 7346 & $\begin{array}{l}\text { regulation of progression } \\
\text { through mitotic cell cycle }\end{array}$ & 3 & 7 & 43 & 3.68 & 0.008 \\
\hline 6607 & $\begin{array}{l}\text { NLS-bearing substrate } \\
\text { import into nucleus }\end{array}$ & 3 & 8 & 38 & 3.34 & 0.013 \\
\hline 15718 & monocarboxylic acid transp & 3 & 8 & 38 & 3.34 & 0.020 \\
\hline 7242 & intracellular signaling casc. & 58 & 566 & 10 & 3.02 & 0.003 \\
\hline 165 & MAPKKK cascade & 10 & 59 & 17 & 2.95 & 0.006 \\
\hline 8643 & carbohydrate transport & 4 & 15 & 27 & 2.95 & 0.019 \\
\hline 6817 & phosphate transport & 9 & 52 & 17 & 2.87 & 0.014 \\
\hline 51169 & nuclear transport & 8 & 49 & 16 & 2.52 & 0.019 \\
\hline 30036 & $\begin{array}{l}\text { actin cytoskeleton } \\
\text { organization \& biogenesis }\end{array}$ & 9 & 58 & 16 & 2.50 & 0.024 \\
\hline 16043 & cell organization and biog. & 62 & 657 & 9 & 2.43 & 0.012 \\
\hline 48518 & + regulation of biol process & 32 & 301 & 11 & 2.42 & 0.011 \\
\hline 19058 & viral infectious cycle & 3 & 12 & 25 & 2.41 & 0.044 \\
\hline 16032 & viral life cycle & 4 & 19 & 21 & 2.37 & 0.036 \\
\hline 43067 & regul. program. cell death & 19 & 161 & 12 & 2.34 & 0.024 \\
\hline 7517 & muscle development & 11 & 80 & 14 & 2.32 & 0.028 \\
\hline 6913 & nucleo-cytoplasmic transp. & 8 & 53 & 15 & 2.27 & 0.029 \\
\hline 6029 & proteoglycan metabolism & 3 & 13 & 23 & 2.24 & 0.067 \\
\hline 48522 & + regulation of cell process & 27 & 254 & 11 & 2.22 & 0.031 \\
\hline 51329 & interphase mitotic cellcycle & 5 & 28 & 18 & 2.21 & 0.046 \\
\hline 7596 & blood coagulation & 8 & 54 & 15 & 2.21 & 0.037 \\
\hline 7599 & hemostasis & 8 & 55 & 15 & 2.15 & 0.042 \\
\hline 30029 & actin filam-based process & 9 & 65 & 14 & 2.12 & 0.055 \\
\hline 42981 & regulation of apoptosis & 18 & 159 & 11 & 2.09 & 0.042 \\
\hline 6820 & anion transport & 12 & 96 & 13 & 2.07 & 0.042 \\
\hline 7243 & protein kinase cascade & 18 & 160 & 11 & 2.06 & 0.057 \\
\hline 42060 & wound healing/coagulation & 8 & 57 & 14 & 2.04 & 0.066 \\
\hline 7254 & JNK cascade & 5 & 30 & 17 & 2.04 & 0.063 \\
\hline 7264 & $\begin{array}{l}\text { small GTPase mediated } \\
\text { signal transduction }\end{array}$ & 13 & 108 & 12 & 2.00 & 0.059 \\
\hline 6928 & cell motility/location of cell & 14 & 119 & 12 & 1.99 & 0.047 \\
\hline
\end{tabular}

GO terms biological process with Z-score $>1.96, \mathrm{p}<0.10$, and number changed $\geq 3$ were considered to be significantly changed. 


\section{Chapter 4}

Table 4. Significantly changed genes in adipogenesis related processes

\begin{tabular}{|c|c|c|}
\hline Gene & Symbol & $\begin{array}{l}\text { Fold change } \\
\text { T2DM / Contr. }\end{array}$ \\
\hline \multicolumn{3}{|l|}{ Adipogenesis } \\
\hline Interferon regulatory factor 1 & IRF1 & $2.06(1.38-3.10)$ \\
\hline Interleukin 6 signal transducer (gp130) & $I L 6 S T$ & $1.42(1.02-1.97)$ \\
\hline CCAAT/enhancer binding protein (C/EBP), delta & CEBPd & $1.39(1.15-1.68)$ \\
\hline Endothelial PAS domain protein 1 & EPAS1 & $1.30(1.20-1.41)$ \\
\hline Kruppel-like factor 5 & KLF5 & $1.29(1.06-1.58)$ \\
\hline Early-b-factor & $E B F$ & $1.21(1.05-1.40)$ \\
\hline Myocyte enhancer factor 2D & MEF2d & $0.90(0.83-0.97)$ \\
\hline Insulin receptor substrate 1 & IRS1 & $0.80(0.65-0.98)$ \\
\hline Insulin growth factor 1 & IGF1 & $0.77(0.62-0.96)$ \\
\hline Transforming growth factor, beta 1 & TGFB1 & $0.75(0.57-0.99)$ \\
\hline Adiponutrin & $A D P N$ & $0.72(0.61-0.86)$ \\
\hline Nuclear receptor interacting protein 1 & NRIP & $0.71(0.56-0.91)$ \\
\hline Hypoxia induced factor 1 alpha & HIF1a & $0.70(0.50-0.98)$ \\
\hline Nuclear receptor co-repressor 2 & NCOR2 & $0.69(0.56-0.84)$ \\
\hline Kruppel-like factor 6 & $K L F 6$ & $0.63(0.49-0.94)$ \\
\hline \multicolumn{3}{|l|}{ Focal adhesion / Extracellular matrix (ECM) / cytoskeleton } \\
\hline Tenascin XB & $T N X B$ & $1.96(1.12-3.43)$ \\
\hline ADAM metallopeptidase, thrombospondin type 1 motif 5 & ADAMTS5 & $1.61(1.08-2.38)$ \\
\hline Mitogen-activated protein kinase kinase 2 & MAP2K2 & $1.25(1.03-1.51)$ \\
\hline Von Willebrand factor & $V W F$ & $1.15(1.03-1.28)$ \\
\hline Matrix metallopeptidase 2 & MMP2 & $0.90(0.86-0.95)$ \\
\hline Actin, gamma 1 & ACTG1 & $0.88(0.81-0.96)$ \\
\hline Growth factor receptor-bound protein 2 & GRB2 & $0.86(0.76-0.97)$ \\
\hline platelet derived growth factor $\mathrm{D}$ & PDGFD & $0.85(0.75-0.96)$ \\
\hline SHC (Src homology 2 domain containing) transform prot 1 & SHC1 & $0.85(0.73-0.98)$ \\
\hline Rho-associated, coiled-coil containing protein kinase 1 & ROCK1 & $0.80(0.66-0.96)$ \\
\hline Insulin-like growth factor 1 (somatomedin C) & $I G F 1$ & $0.77(0.62-0.96)$ \\
\hline Integrin, alpha 11 & ITGA11 & $0.76(0.63-0.92)$ \\
\hline Actin, beta & ACTB & $0.72(0.58-0.90)$ \\
\hline Integrin, alpha $\mathrm{V}$ & ITGAV & $0.71(0.59-0.86)$ \\
\hline Protein phosphatase 1 , regulatory (inhibitor) subunit $12 a$ & $P P P 1 R 12 a$ & $0.68(0.58-0.81)$ \\
\hline Vascular endothelial growth factor $A$ & VEGF & $0.68(0.48-0.95)$ \\
\hline A desintegrin and metallopeptidase domain 12 & ADAM12 & $0.66(0.49-0.89)$ \\
\hline 3-phosphoinositide dependent protein kinase-1 & $P D P K 1$ & $0.65(0.47-0.89)$ \\
\hline Integrin, alpha 5 (fibronectin receptor, alpha polypeptide) & ITGA5 & $0.65(0.48-0.86)$ \\
\hline Cyclin D1 & CCND1 & $0.63(0.42-0.93)$ \\
\hline Collagen, type $\mathrm{V}$, alpha 1 & COL5A1 & $0.58(0.44-0.75)$ \\
\hline Actinin, alpha 1 & ACTN1 & $0.57(0.45-0.73)$ \\
\hline Tenascin C (hexabrachion) & $T N C$ & $0.50(0.29-0.86)$ \\
\hline Collagen, type IV, alpha 1 & COL4A1 & $0.47(0.33-0.66)$ \\
\hline Collagen, type XI, alpha 1 & COL11A1 & $0.41(0.23-0.71)$ \\
\hline Matrix metallopeptidase 1 & MMP1 & $0.33(0.15-0.73)$ \\
\hline \multicolumn{3}{|l|}{ MAPK signaling pathway } \\
\hline Caspase 1 , apoptosis-related cysteine peptidase & CASP1 & $1.34(1.01-1.77)$ \\
\hline
\end{tabular}




\begin{tabular}{|c|c|c|}
\hline Protein phosphatase $1 \mathrm{~B}$ (formerly $2 \mathrm{C}$ ) & $P P M 1 B$ & $1.32(1.09-1.59)$ \\
\hline Mitogen-activated protein kinase kinase 2 & $M A P 2 K 2$ & $1.25(1.03-1.51)$ \\
\hline Heat shock $70 \mathrm{kDa}$ protein $1 \mathrm{~A}$ & HSPA1A & $1.21(1.01-1.45)$ \\
\hline Nerve growth factor, beta polypeptide & $N G F B$ & $1.20(1.01-1.43)$ \\
\hline Growth factor receptor-bound protein 2 & GRB2 & $0.86(0.76-0.97)$ \\
\hline Mitogen-activated protein kinase kinase kinase kinase 3 & MAP4K3 & $0.79(0.65-0.96)$ \\
\hline Transforming growth factor, beta 1 & TGFB1 & $0.75(0.57-0.99)$ \\
\hline mitogen-activatged protein kinase kinase kinase 4 & MAP3K4 & $0.68(0.47-0.97)$ \\
\hline mitogen-activated protein kinase 8 interacting protein 3 & MAPK8IP3 & $0.67(0.53-0.84)$ \\
\hline Ras oncogene homolog & MRAS & $0.71(0.56-0.91)$ \\
\hline Interleukin 1 , beta & $I L 1 B$ & $0.58(0.36-0.94)$ \\
\hline Dual specificity protein phosphatase 10 & DUSP10 & $0.58(0.43-0.78)$ \\
\hline \multicolumn{3}{|l|}{ Insulin signaling } \\
\hline ADP-ribosylation facto & $A R F 1$ & $1.35(1.02-1.77)$ \\
\hline Phosphoinositide-3-kinase, regulatory subunit $3(p 55, y)$ & PIK3A3 & $1.32(1.21-1.44)$ \\
\hline Mitogen-activated protein kinase kinase 2 & MAP2K2 & $1.25(1.03-1.51)$ \\
\hline Flotillin 1 & FLOT1 & $1.23(1.02-1.49)$ \\
\hline $\mathrm{RAB} 4 \mathrm{~A}, \mathrm{me}$ & $R A B 4 A$ & $1.14(1.02-1.25)$ \\
\hline Phosphoino & PIK $3 C 2 A$ & $0.89(0.79-0.99)$ \\
\hline Inositol polyphosphate phosphatase-like 1 & INPPL1 & $0.88(0.80-0.97)$ \\
\hline Growth factor receptor-bound protein 2 & GRB2 & $0.86(0.76-0.97)$ \\
\hline Mitogen-activat & MAP $3 K 3$ & $0.86(0.75-0.99)$ \\
\hline SHC (Src homology 2 domain containing) transform prot 1 & SHC1 & $0.85(0.73-0.98)$ \\
\hline Syntaxin binding protein 1 & $S T X B P 1$ & $0.85(0.73-0.99)$ \\
\hline n family, member 4 & TBC1D4 & $0.84(0.79-0.89)$ \\
\hline Insulin receptor substrate 1 & IRS1 & $0.80(0.65-0.98)$ \\
\hline Mitogen-activated protein kinase kinase kinase kinase 3 & MAP4K3 & $0.79(0.65-0.96)$ \\
\hline Protein tyrosine phosphatase, non-receptor type 1 & PTPN1 & $0.78(0.63-0.96)$ \\
\hline Solute carrier family 2 (glucose transporter), member 1 & $S L C 2 A 1$ & $0.76(0.61-0.96)$ \\
\hline Mitogen-activated protein kinase kinase kinase kinase 2 & MAP4K2 & $0.74(0.60-0.93)$ \\
\hline 3-phosphoinositide dependent protein kinase-1 & $P D P K 1$ & $0.65(0.74-0.89)$ \\
\hline Insulin induced gene 11 & INSIG1 & $0.37(0.29-0.48)$ \\
\hline
\end{tabular}

Data are means \pm confidence interval (CI)

Differentially expressed processes: Adipogenesis, Insulin signaling, Lipid metabolism and Inflammation and Apoptosis

Pathways comprising genes involved in the adipogenesis process, MAPK signaling pathway, Focal adhesion pathway and insulin signaling were among the most pronounced pathways in the GenMAPP/MAPPFinder analysis (Table 2). Genes involved in these pathways (Table 4) are mainly decreased. Insulin signaling pathway shows reduced insulin receptor substrate regulation, MEK/MAP kinase activation and modulators of insulin signaling. Also lipid metabolism, including genes involved in lipid $\beta$-oxidation, biosynthesis and lipolysis were decreased in T2DM preadipocytes. The hormone sensitive lipolysis (HSL) process was 


\section{Chapter 4}

not clearly represented in GenMAPP, but by performing a literature search of significantly changed genes, we identified changes in key regulators of CAMP-dependent protein kinase induction of $\mathrm{HSL}$, like ADRA2A and PKA, leading to reduced HSL.

Although preadipocytes are metabolically not very active, the key gene for the import of long chain fatty acids into mitochondria and $\beta$ oxidation, CPT1, was severely decreased. CPT1a (liver isoform) was found decreased with both microarray and QPCR analysis in preadipocytes of T2DM preadipocytes, while CPT1b (muscle isoform) expression was unchanged. To test if the reduced CPT1a expression results in reduced CPT1 activity, the CPT1 activity was measured by tandem mass spectrometry, but no significant difference could be detected between the T2DM $(4,779 \pm 587 \mathrm{pmol} / \mathrm{mg} . \mathrm{min})$ and control preadipocytes $(4,139 \pm 987 \mathrm{pmol} / \mathrm{mg} \cdot \mathrm{min})$. To explore the expression pattern of CPT1a and CPT1b during adipogenesis, preadipocytes were differentiated to adipocytes. Since human preadipocytes generally have low differentiation capacity, a preadipocyte cell-line derived from a patient with Simpson-Golabi-Behmel Syndrome (SGBS) with a high differentiation capacity and a gene expression pattern which is similar to differentiating preadipocytes from healthy subjects [27], were cultured and differentiated according to the protocol. Expression of CPT1a and $C P T 1 b$ was analysed by qPCR analysis (Fig. 2a), and TBP was used for normalization. The first day after induction of adipogenesis, CPT1a expression increases and is the predominant form. From day 2 to 4 CPT1a decreases again, whereas CPT1b expression goes up after induction until day 4 . After day 4 the expression of both genes stabilizes at a more or less equal level. FABP4, which is a marker for adipogenesis, was used to analyze differentiation progression, and FABP4 expression rises drastically during differentiation (Fig. 2b).

Genmapp/Mappfinder analysis (Table 2) further identified the local map Complement and Coagulation cascade, and GO terms (Table 3) Regulation of programmed cell death, Regulation of apoptosis and Caspase regulator activity to be significantly overrepresented, indicating stimulation of the process. 


\section{Discussion}

Gene expression profiling of cultured preadipocytes from T2DM and a control group was performed to identify altered molecular processes related to T2DM. Before analyzing the gene expression data in full detail, we first checked the consistency of our results and the homogeneity of the preadipocyte cultures. Microarray data were validated by qPCR analysis of 20 genes that were significantly changed on the microarray platform (Fig. 1). All transcripts validated by qPCR analysis showed a fold change in the same direction on both platforms, although this was non-significant for 7 out of the 20 genes. Analysis of microarray and GPCR data were performed in a similar way, but differences between the platforms, e.g. probe location, dye swap, normalization and relative small fold changes (fold change $<2$ ), might explain why part of the qPCR results did not reach the same level of significance. The correlation between the microarray data and qPCR data was $0.82(p<0.01)$, indicating that the obtained microarray data is accurate and valid [28]. From checking the preadipocyte cultures visually and by analyzing cell-type specific gene expression (ESM Table 2 [19]) we concluded that the results were derived from preadipocyte specific gene expression without significant contribution of macrophage, stem cell or endothelial cell infiltration. This allows identifying intrinsic alterations in molecular mechanisms by excluding the environmental influence. Culturing did not influence the ability to detect altered gene expression related to the diseased state $[13,29]$. Additionally, wash-out of medication, e.g. metformin, is expected during culture, since it acts for only 8-12 hours and adipose tissue is not the primary target. Therefore, we do not expect that metformin therapy influences our data. Insulin was used by 3 T2DM patients, but the insulin and glucose levels were comparable with T2DM subjects withdrawn from therapy. We conclude that our study reflects reliable gene expression differences between preadipocytes from T2DM and controls.

\section{Differentiation capacity of T2DM preadipocytes}

We observed a general decrement of genes involved in transcription regulation of the adipogenesis process, including insulin signaling, transcription regulation, and extracellular matrix (ECM) and cytoskeleton remodeling in T2DM preadipocytes (Figure 3). Transcription activation 


\section{Chapter 4}

by insulin/IGF signaling is one of the first events upon induction of differentiation, and IRS1 and IGF1, which are the stimulators of this process, are decreased in T2DM preadipocytes. IGF1 reduction results in decreased expression of downstream mitogen activated protein kinases (MAPKs) and transcription factor KLF6, which is induced by IGF1 and inhibits delta like kinase 1 ( $D L K 1)$, and decrement of $K L F 6$ expression inhibits adipogenesis $[30,31]$. The fact that the expression of IGFR1 and $D L K 1$ is not changed may imply that these proteins are under posttranslational control. Preadipocytes from T2DM preadipocytes have increased expression of TWIST2, which interacts with sterol regulatory elements binding protein 1 (SREBP1) and thereby inhibits transcriptional activity of SREBP1. Overexpression of TWIST2 may inhibit processes activated by $S R E B P 1$, a key transcription factor in lipogenesis and insulin-dependent gene expression during adipogenesis. In contrast, $C / E B P \delta$ and its downstream target KLF5 are stimulators of adipogenesis that are increased in T2DM preadipocytes [32,33]. Although C/EBPס is a downstream target of $S R E B P$, upon insulin stimulation, $82 \%$ of the effect is achieved via $C / E B P \beta$, which is expressed during adipogenesis [34]. $C / E B P \delta$ has also been implicated in inflammation, and can be activated by e.g. TNF $\alpha$, INF- $\gamma, \mathrm{IL}-1$ and IL-6 [35]. Increased CEBP $\delta$ expression may therefore be related to inflammation rather than insulin-stimulated differentiation. In addition, preadipocytes of T2DM subjects have increased expression of inhibitors of adipogenesis, like IL6st and IRF1. IL6st overexpression was shown to inhibit adipogenesis by affecting Ras/STAT signaling [36] and IRFs play key roles in immune response, but they have been recently also identified as inhibitors of adipogenesis [37]. Morphological changes, when the fibroblast-like shape changes into a spherical shape, mostly occur in the first 12 hours of differentiation and are associated with ECM and cytoskeletal changes $[32,38]$. A number of genes encoding ECM and cytoskeletal constituents showed altered expression in T2DM preadipocytes, like decreased expression of collagens, integrins, actins and tropomyosins, and an increment of fibronectin (Fig 3, Table 4). ECM and cytoskeletal modifiers like MMP1, MMP2 and ADAM12 were also down-regulated in T2DM preadipocytes, only ADAMTS5 was up-regulated in T2DM preadipocytes, but its exact function in adipogenesis is still unknown. Taken together, the expression profile of T2DM preadipocytes suggests a decreased differentiation capacity. This is in line with previous studies that showed decreased expression of key adipogenesis regulators that are induced 
after induction of adipogenesis, e.g. PPAR,$C / E B P \beta$ and SREBP1, and a reduced formation of new adipocytes in T2DM subjects and mouse models [39-41]. Additionally, the importance of insulin signaling in adipogenesis is exemplified by the fact that expression of cytoskeleton, adhesion and ECM components is up-regulated by insulin treatment of mouse 3T3-L1 preadipocytes [42-44]. Previous studies have suggested that decreased adipogenesis in diabetic subjects could be a result of altered adipokine signalling or inflammatory cytokines [45]. This cannot be observed in our data since those studies were conducted on adipose tissue or isolated adipocytes while our study focussed on cultured preadipocytes, which allowed us to detect intrinsic changes leading to decreased adipogenesis capacity. Whether this is the consequence of insulin resistance in T2DM due to altered signalling of macrophages and adipocytes, or that decreased differentiation capacity predisposes to it, is yet unclear. In contrast to the general down-regulation of the adipogenesis process, expression of genes involved in adipogenesis, which are stimulated by inflammation, were up-regulated in T2DM preadipocytes. This shows that adipogenesis involves multiple regulators and the relative effect of the regulators has to be studied more closely to determine key regulators that might define disease progression.

\section{Lipid metabolism}

Expression of genes that play a role in lipid metabolism, including lipid $\beta$-oxidation, HSL-stimulated lipolysis and lipogenesis were downregulated in T2DM preadipocytes. CPT1a expression was down-regulated in T2DM patients, but did not result in a reduced CPT1 activity, indicating that the reduced expression does not affect lipid import into mitochondria for $\beta$-oxidation. Since, CPT1a is first up-regulated at the induction of adipogenesis and then expression switches from mainly CPT1a towards expression of both CPT1a and CPT1b (Fig. 2) [46], the reduced expression of CPT1a in T2DM preadipocytes could be involved in preadipocyte differentiation rather than metabolism in preadipocytes. The reduced expression of genes involved in HSL-mediated lipolysis is in agreement with previous reports in insulin resistant preadipocytes and adipocytes [47]. However, since preadipocytes are not metabolically very active, the effect of reduced lipolysis can also be a consequence of decreased expression of key regulators of adipogenesis in T2DM, like $P P A R Y$ and SREBP1. These intrinsic changes in T2DM preadipocyte lipid metabolism might therefore contribute to the altered adipogenesis 


\section{Chapter 4}

process, but it could also have an effect on the metabolism of fully differentiated adipocytes.

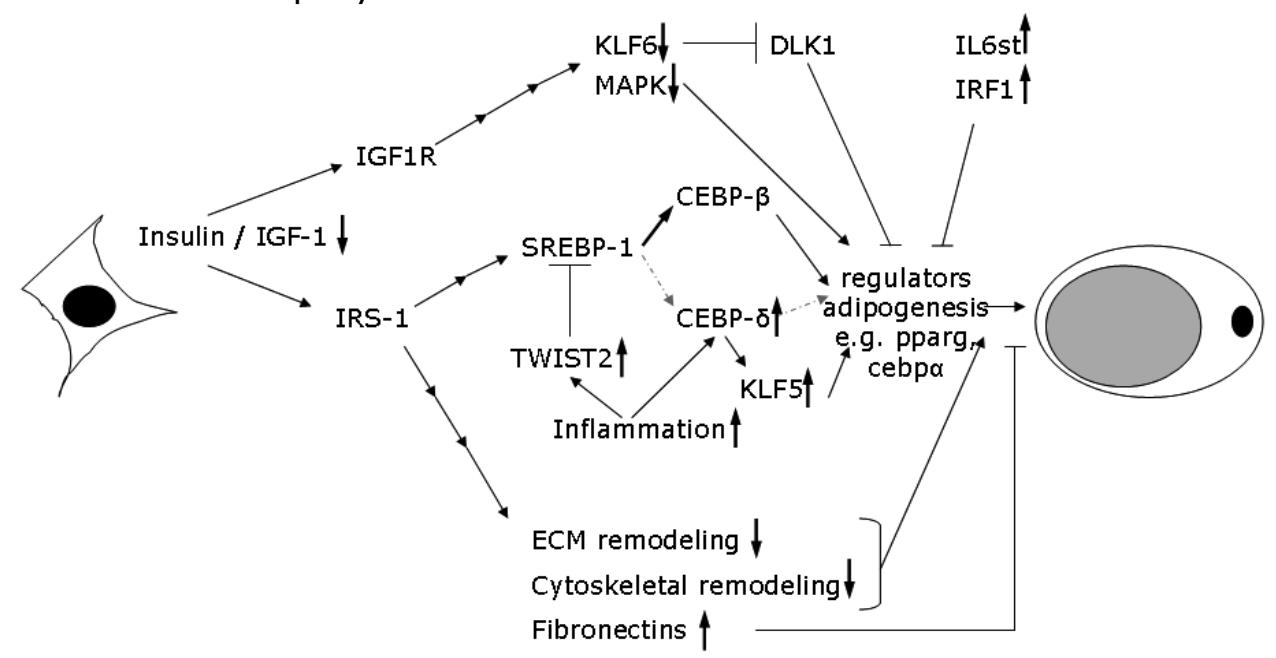

Fig. 3. Schematic overview of the most significant gene expression changes in the adipogenesis process of T2DM preadipocytes. Faced up arrows indicate up-regulated and faced down arrows indicate down-regulated gene expression in T2DM preadipocytes. Genes or processes without arrows had no altered expression pattern. The main preferred routes are depicted in solid lines and dashed lines indicate alternative routes. ECM and cytoskeletal remodelling are down-regulated, based on reduced expression in T2DM of COL4a1, COL5a1, COL11a1, ITGAV, MMP2, ADAM12 like, ACTN1, ACTB, TPM1 and TPM2. Fibronectins (FNDC1, FLRT2) were up-regulated. Inflammation may be induced by e.g. TNFa and INF- $\gamma$. CEPB- $\beta$ and the regulators of adipogenesis are activated during the differentiation process itself and not in the preadipocytes.

\section{Inflammation and apoptosis}

Processes associated with complement and caspase activation and regulation of apoptosis/programmed cell death were overrepresented in T2DM preadipocytes. Anti-apoptotic gene expression was decreased and pro-apoptotic gene expression was increased. Inflammation-related gene expression has been observed in adipocytes and preadipocytes from obese Pima Indians when compared to non-obese Pima Indians $[13,16]$. Inflammation is regarded as a general characteristic of obesity, but gene expression analysis suggests a tendency to enhanced apoptosis in preadipocytes of T2DM subjects. However, no significant change in growth of the T2DM preadipocytes were observed due to enhanced apoptosis. Furthermore accurate experiments, like apoptosis assays and growth curves, should clarify the role and significance of apoptosis in preadipocytes of T2DM patients. 
In conclusion, several molecular pathways are altered in cultured T2DM preadipocytes, providing a first comprehensive overview of the molecular processes involved. This gives a possible explanation for the decreased formation of new adipocytes and enlarged adipocytes in patients with T2DM, which contribute to increased ectopic fat deposition. Additionally, we have demonstrated that these alterations are not due to signalling from other cell-types. Gaining insight in het molecular mechanism of adipogenesis may lead to identification of targets for therapeutic interventions, aiming at enhancing adipocyte differentiation and metabolism to improve lipid storage capacity, which in consequence could reduce ectopic fat depositioning and enhance insulin sensitivity.

\section{Acknowledgements}

We would like to thank Martin Wabitsch for kindly providing the SGBS cells, Glaxo Smith Kline for kindly providing Rosiglitazone, and the Microarray Facility Utrecht for providing the oligonucleotide microarrays. The research presented was funded by the Dutch Diabetes Foundation (DFN 2004.00.040) 


\section{References}

1. Sell, H., J. Eckel, and D. Dietze-Schroeder, Pathways leading to muscle insulin resistance--the muscle--fat connection. Arch Physiol Biochem, 2006. 112(2): p. 105-13.

2. King, H., R.E. Aubert, and W.H. Herman, Global burden of diabetes, 1995-2025: prevalence, numerical estimates, and projections. Diabetes Care, 1998. 21(9): p. 1414-31.

3. Bloomgarden, Z.T., American Diabetes Association Annual Meeting, 1999: diabetes and obesity. Diabetes Care, 2000. 23(1): p. 118-24.

4. P. Puska, C.N., D. Porter. Obesity and overweight. World Health Organization [cited 2009; Available from: http://www.who.int/dietphysicalactivity/publications/ facts/obesity/en/.

5. Jazet, I.M., H. Pijl, and A.E. Meinders, Adipose tissue as an endocrine organ: impact on insulin resistance. Neth J Med, 2003. 61(6): p. 194-212.

6. Heilbronn, L., S.R. Smith, and E. Ravussin, Failure of fat cell proliferation, mitochondrial function and fat oxidation results in ectopic fat storage, insulin resistance and type II diabetes mellitus. Int J Obes Relat Metab Disord, 2004. 28 Suppl 4: p. S12-21.

7. Goralski, K.B. and C.J. Sinal, Type 2 diabetes and cardiovascular disease: getting to the fat of the matter. Can J Physiol Pharmacol, 2007. 85(1): p. 113-32.

8. Kahn, B.B. and J.S. Flier, Obesity and insulin resistance. J Clin Invest, 2000. 106(4): p. 473-81.

9. Hajer, G.R., T.W. van Haeften, and F.L. Visseren, Adipose tissue dysfunction in obesity, diabetes, and vascular diseases. Eur Heart J, 2008. 29(24): p. 2959-71.

10. Cinti, S., The adipose organ. Prostaglandins Leukot Essent Fatty Acids, 2005. 73(1): p. 9-15.

11. Lundgren, M., et al., Fat cell enlargement is an independent marker of insulin resistance and 'hyperleptinaemia'. Diabetologia, 2007. 50(3): p. 625-33.

12. Yang, X., et al., Evidence of impaired adipogenesis in insulin resistance. Biochem Biophys Res Commun, 2004. 317(4): p. 1045-51.

13. Nair, S., et al., Increased expression of inflammation-related genes in cultured preadipocytes/stromal vascular cells from obese compared with non-obese Pima Indians. Diabetologia, 2005. 48(9): p. 1784-8.

14. Hammarstedt, A., et al., Wnt-signaling is maintained and adipogenesis inhibited by TNFalpha but not MCP-1 and resistin. Biochem Biophys Res Commun, 2007. 357(3): p. 700-6.

15. Dahlman, I., et al., Downregulation of electron transport chain genes in visceral adipose tissue in type 2 diabetes independent of obesity and possibly involving tumor necrosis factor-alpha. Diabetes, 2006. 55(6): p. 1792-9.

16. Lee, Y.H., et al., Microarray profiling of isolated abdominal subcutaneous adipocytes from obese vs non-obese Pima Indians: increased expression of inflammation-related genes. Diabetologia, 2005. 48(9): p. 1776-83.

17. Eurlings, P.M., et al., Identification of differentially expressed genes in subcutaneous adipose tissue from subjects with familial combined hyperlipidemia.

J Lipid Res, 2002. 43(6): p. 930-5. 


\section{Intrinsic gene expression changes in T2DM preadipocytes}

18. Troost, F.J., et al., Identification of the transcriptional response of human intestinal mucosa to Lactobacillus plantarum WCFS1 in vivo. BMC Genomics, 2008. 9: p. 374.

19. Van Tienen, F.H. Supplementary material thesis. 2010 [cited; Available from: http://www.personeel.unimaas.nl/florence-vantienen/.

20. Akaike, $\mathrm{H}$. Information theory and an extension of the maximum likelihood principle. in Second International Symposium on Inference Theory. 1973. Budapest: Akadémiai Kiadó.

21. Salomonis, N., et al., GenMAPP 2: new features and resources for pathway analysis. BMC Bioinformatics, 2007. 8: p. 217.

22. Doniger, S.W., et al., MAPPFinder: using Gene Ontology and GenMAPP to create a global gene-expression profile from microarray data. Genome Biol, 2003. 4(1): p. R7.

23. Ashburner, M., et al., Gene ontology: tool for the unification of biology. The Gene Ontology Consortium. Nat Genet, 2000. 25(1): p. 25-9.

24. Ihaka R, G., R "R: a language for data analysis and graphics". Journal of Computational Graphics and Statistics, 1996. 5(3): p. 299-314.

25. Lindsey, J., Models for repeated measurements, 2nd edition. 1999, Oxford: Oxford University Press. 536.

26. van Vlies, N., et al., An improved enzyme assay for carnitine palmitoyl transferase $I$ in fibroblasts using tandem mass spectrometry. Mol Genet Metab, 2007. 90(1): p. 24-9.

27. Wabitsch, M., et al., Characterization of a human preadipocyte cell strain with high capacity for adipose differentiation. Int J Obes Relat Metab Disord, 2001. 25(1): p. 8-15.

28. Morey, J.S., J.C. Ryan, and F.M. Van Dolah, Microarray validation: factors influencing correlation between oligonucleotide microarrays and real-time PCR. Biol Proced Online, 2006. 8: p. 175-93.

29. Meex, S.J., et al., Up-regulation of CD36/FAT in preadipocytes in familial combined hyperlipidemia. Faseb J, 2005. 19(14): p. 2063-5.

30. Li, D., et al., Kruppel-like factor-6 promotes preadipocyte differentiation through histone deacetylase 3-dependent repression of DLK1. J Biol Chem, 2005. 280(29): p. 26941-52.

31. Bentov, I., et al., Insulin-like growth factor-i regulates Kruppel-like factor-6 gene expression in a p53-dependent manner. Endocrinology, 2008. 149(4): p. 1890-7.

32. Hackl, H., et al., Molecular processes during fat cell development revealed by gene expression profiling and functional annotation. Genome Biol, 2005. 6(13): p. R108.

33. Oishi, Y., et al., Kruppel-like transcription factor KLF5 is a key regulator of adipocyte differentiation. Cell Metab, 2005. 1(1): p. 27-39.

34. Le Lay, S., et al., Insulin and sterol-regulatory element-binding protein-1c (SREBP-1C) regulation of gene expression in 3T3-L1 adipocytes. Identification of CCAAT/enhancer-binding protein beta as an SREBP-1C target. J Biol Chem, 2002. 277(38): p. 35625-34.

35. Ramji, D.P. and P. Foka, CCAAT/enhancer-binding proteins: structure, function and regulation. Biochem J, 2002. 365(Pt 3): p. 561-75.

36. Miyaoka, Y., et al., Oncostatin $M$ inhibits adipogenesis through the RAS/ERK and STAT5 signaling pathways. J Biol Chem, 2006. 281(49): p. 37913-20. 


\section{Chapter 4}

37. Eguchi, J., et al., Interferon regulatory factors are transcriptional regulators of adipogenesis. Cell Metab, 2008. 7(1): p. 86-94.

38. Gregoire, F.M., Adipocyte differentiation: from fibroblast to endocrine cell. Exp Biol Med (Maywood), 2001. 226(11): p. 997-1002.

39. Dubois, S.G., et al., Decreased expression of adipogenic genes in obese subjects with type 2 diabetes. Obesity (Silver Spring), 2006. 14(9): p. 1543-52.

40. Wang, M.Y., et al., Adipogenic capacity and the susceptibility to type 2 diabetes and metabolic syndrome. Proc Natl Acad Sci U S A, 2008. 105(16): p. 6139-44.

41. Tseng, Y.H., et al., Prediction of preadipocyte differentiation by gene expression reveals role of insulin receptor substrates and necdin. Nat Cell Biol, 2005. 7(6): p. 601-11.

42. Wang, P., et al., Insulin modulates the secretion of proteins from mature 3T3-L1 adipocytes: a role for transcriptional regulation of processing. Diabetologia, 2006. 49(10): p. 2453-62.

43. Vu, T.H. and Z. Werb, Matrix metalloproteinases: effectors of development and normal physiology. Genes Dev, 2000. 14(17): p. 2123-33.

44. Kawaguchi, N., et al., ADAM12 induces actin cytoskeleton and extracellular matrix reorganization during early adipocyte differentiation by regulating beta1 integrin function. J Cell Sci, 2003. 116(Pt 19): p. 3893-904.

45. Janke, J., et al., Mature adipocytes inhibit in vitro differentiation of human preadipocytes via angiotensin type 1 receptors. Diabetes, 2002. 51(6): p. 1699707.

46. Brown, N.F., et al., Mouse white adipocytes and 3T3-L1 cells display an anomalous pattern of carnitine palmitoyltransferase (CPT) I isoform expression during differentiation. Inter-tissue and inter-species expression of CPT I and CPT II enzymes. Biochem J, 1997. 327 ( Pt 1): p. 225-31.

47. Jocken, J.W., et al., Adipose triglyceride lipase and hormone-sensitive lipase protein expression is decreased in the obese insulin-resistant state. J Clin Endocrinol Metab, 2007. 92(6): p. 2292-9. 


\section{Chapter}

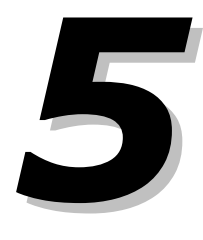

Wnt5b stimulates adipogenesis by activating PPARY, and inhibiting the $\beta$-catenin dependent Wnt signalling pathway together with Wnt5a

F.H.J. van Tienen, H. Laeremans, C.J.H. van der Kallen, H.J.M. Smeets 


\section{Chapter 5}

\section{Abstract}

Correct Wnt signalling is required for adipogenesis and alterations occur in Type 2 Diabetes Mellitus (T2DM). Gene expression studies showed that $\beta$-catenin independent $W n t 5 b$ was down-regulated in T2DM preadipocytes, while its paralog $W n t 5 a$ was unchanged. Our study aimed at defining the expression profile and function of Wnt5a and Wnt5b during adipogenesis by determining their effect on $A P 2$ and PPARY expression and assessing the level of $\beta$-catenin translocation in mouse 3T3-L1 preadipocytes. Additionally, we explored the effect on adipogenic capacity by $W n t 5 b$ overexpression in combination with stimulation of the $\beta$-catenin dependent or $\beta$-catenin independent Wnt signalling. Expression of $W n t 5 b$ was, like Wnt5a, down-regulated upon induction of differentiation and both inhibit $\beta$-catenin dependent Wnt signalling at the initiation of adipogenesis. Wnt5b additionally appears to be a potent enhancer of adipogenic capacity by stimulation of PPARY and $a P 2$. Down-regulation of $W n t 5 b$ could therefore contribute to decreased adipogenesis observed in T2DM diabetic subjects.

\section{Introduction}

Gene expression analysis of preadipocytes of Type 2 Diabetes Mellitus (T2DM) patients displayed decreased expression of a number of genes involved in the formation of new adipocytes, called adipogenesis, when compared to age- and BMI matched controls. One of the down-regulated genes was WNT5b, which is a member of the Wnt family. Expression of WNT5a, which is a paralog of WNT5b, was unchanged [1]. As T2DM is associated with decreased adipogenesis and altered Wnt expression [2], we decided to study the role WNT5 $b$ and WNT5a during adipogenesis in further detail.

The Wnt family is an evolutionarily conserved family of secreted glycoproteins with well-established roles in cellular proliferation, differentiation, and polarity during embryogenesis as well as control homeostatic self-renewal in a number of adult tissues [3, 4]. Wnt membership is based on sequence rather than functional properties. Functionally, the Wnt family can be divided into at least two groups, the $\beta$-catenin dependent Wnt pathway, which activates target genes through 
stabilization of $\beta$-catenin, and the $\beta$-catenin independent or aspecific Wnt pathway that is independent of $\beta$-catenin and activates gene transcription e.g. by stimulation of the intracellular calcium flux leading to activation of $\mathrm{Ca}^{2+}$-dependent effectors, like activation of phospholipase $C$ and protein kinase $C$ (PKC).

The $\beta$-catenin dependent Wnt-signaling pathway has been reported to inhibit adipogenesis e.g. by Wnt10b or Wnt3a [5-7]. On the other hand, $\beta$-catenin independent Wnt genes like Wnt4, Wnt5a, Wnt5b have been proposed as stimulators of adipogenesis [8]. Expression of $\beta$-catenin independent Wnt5a has a stimulatory effect in the early phase, since Wnt5a gets down-regulated 12 hours after induction of differentiation in 3T3-L1 mouse preadipocytes, and knock-down of Wnt5a results in decreased adipogenesis and reduced expression of key regulators of adipogenesis, like peroxisome proliferator activated receptor $\mathrm{Y}$ (PPARY) and CCAAT/enhancer binding protein a (CEBPa) [8, 9]. In contrast, $W n t 5 b$, which is a paralog of Wnt5a, is reported to be up-regulated during adipogenesis with the highest expression at day two, and overexpression of $W n t 5 b$ significantly stimulates adipogenesis in murine preadipocytes $[10,11]$.

Since $W N T 5 b$, and not WNT5a, was down-regulated in preadipocytes of T2DM patients, our aim was to explore the expression pattern of both Wnt5b and Wnt5a during adipogenesis, using mouse 3T3-L1 preadipocytes as model for adipogenesis. In addition, the effect of $W n t 5 b$ on murine adipogenesis was studied under conditions in which either the $\beta$-catenin dependent Wnt pathway or the $\beta$-catenin independent Wnt pathway was stimulated.

\section{Materials and Methods}

\section{Sequence alignment}

Nucleotide and protein sequence alignment were performed using the Clustal W multiple sequence alignment program [12]. mRNA sequence alignment of mouse Wnt5a mRNA NM_009524.2 and Wnt5b NM_009525.2 mRNA, and human WNT5a NM_03392.3 and the 2 isoforms of human WNT5b NM_032642.2 and NM_030775.2 were performed. On protein level, mouse Wnt5a NP_033550, human WNT5a 


\section{Chapter 5}

NP_003383, rat Wnt5a NP_072153, mouse Wnt5b NP_033551, human WNT5b NP_110402 and rat Wnt5b XP_001057561 were analyzed.

\section{Cell culture}

Mouse 3T3-L1 preadipocytes (ATCC) were grown in Dulbecco's modified Eagle's medium (DMEM) (Gibco) containing 10\% FCS (Gibco) and penicillin/streptavidin (Gibco). Differentiation was induced in two-days post-confluence by changing medium with DMEM containing $0.5 \mathrm{mM} 3-$ isobutyl-1-methylxantine (IBMX) (Sigma), $10 \mu \mathrm{g} / \mathrm{ml}$ insulin (Sigma) and $1 \mu \mathrm{M}$ dexamethasone (Sigma). After two days, medium was replaced with DMEM containing $10 \%$ FCS and $10 \mu \mathrm{g} / \mathrm{ml}$ insulin, and changed every two days.

Wnt3a conditioned medium (Wnt3a-CM), Wnt5a conditioned medium (Wnt5a-CM), and L-cell control medium were prepared in DMEM with $2 \% \mathrm{HS}$ (Invitrogen). Cells were plated on day 0 with a dilution of $1 / 10$. Conditioned medium was collected on days 4 and 7 . Both batches were mixed and filtered before use. The media was used at a final concentration of $20 \%$ in all experiments. Medium of two days confluent 3T3-L1 cells was replaced with DMEM $10 \%$ FCS containing $20 \%$ Wnt3aCM, Wnt5a-CM or L-cell control medium. After 8 hours, media was replaced by differentiation medium containing $20 \%$ Wnt3a-CM, Wnt5aCM or L-cell control medium.

\section{Transfection}

3T3-L1 preadipocytes were transfected at 70\% confluence with PSPORTWnt5b (Openbiosystems) or pcDNA3.1 empty vector with Fugene HD transfection reagents (Roche) according to the manufacturers' protocol.

\section{Quantitative real-time $P C R$ validation}

RNA isolation and DNAse treatment were performed with the high pure RNA isolation kit (Roche) according to the manufacturers' protocol. cDNA was generated from $1 \mu \mathrm{g}$ RNA in a standard reverse transcriptase reaction using M-MulV reverse transcriptase (Finzymes). Primers were designed using Primer Express ${ }^{\circ}$ software version 3.0 (Applied Biosystems) or derived from literature. Wnt5b forward primer 5'-

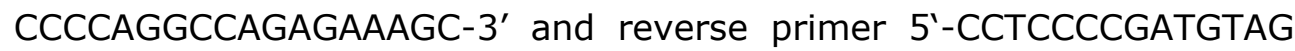
GACAT-3'; WNT5a forward primer 5'-ACTTCGAGAGGCTCCCAGAAC-3' and reverse primer 5'-CTGGGAAAGGAGTGAAGCAAA-3'; aP2 5'GCGTGGAATTC GATGAAATCA-3' and reverse primer 5'- 
CCCGCCATCTAGGGTTATGA-3' [13]; NIk 'CCCAACCGAAGCATTTCAGT' and 'CCGACCTCTGAGATTGTACCTTT C-3'; PPARY 5'TGCCAAAAATATCCCTGGTTTC-3' and reverse primer 5'GGAGGCCAGCATCGTGTAGA-3'. Quantification of transcripts was carried out using the ABI $7900 \mathrm{HT}$ Real-Time PCR detection system using Eurogentec qPCR Mastermix Plus for SYBR Green ${ }^{\circledR}$ I. The cycling conditions were: an initial step for $2^{\prime}$ at $50^{\circ} \mathrm{C}$, activation of the Hot Goldstar enzyme at $95^{\circ} \mathrm{C}$ for $10^{\prime}, 40$ cycles of $15^{\prime \prime}$ at $95^{\circ} \mathrm{C}$ followed by $1^{\prime}$ at $60^{\circ} \mathrm{C}$ (denaturation, annealing, and elongation). The mRNA levels of each gene were normalized to those of the housekeeping gene encoding Cyclophilin A ( СуpA). Statistic analysis was performed using Student Ttest.

\section{Western blots}

For Western blot, nuclear and cytoplasmatic protein extracts were isolated using NE-PER reagent according to the manufacturers protocol (Pierce Biotechnology Inc.). Protein content was measured using the BCA protein assay (Pierce); $20 \mu \mathrm{g}$ of total protein was denatured by boiling in Laemmli sample buffer (BioRad), separated on a $10 \%$ SDSpage gel, and transferred onto a Hybond $C$ nitrocellulose membrane (Amersham Biosciences). After blocking ( $5 \%$ non-fat dry milk (BioRad), $0.1 \%$ Tween in TBS) for $1 \mathrm{~h}$, membranes were incubated overnight at $4^{\circ} \mathrm{C}$ with primary antibodies directed against $\beta$-catenin $1 / 2000$ (BD Biosciences) and $\beta$-actin 1/2000 (Sigma). Anti-mouse immunoglobulin G $1 / 5000$ (Vector Labs Inc.) was used as the secondary antibody, and the membranes were developed using the Western blotting luminal reagent for chemiluminescence detection (Santa Cruz), which was detected with the Chemidoc XRS. Analysis of the images was performed independently by two persons with the Quantity 1-D analysis software (Roche). 


\section{Chapter 5}

\section{Results}

Wnt5a and Wnt5b homology and expression pattern during 3T3-L1 differentiation

To explore the role of WNT5b and its paralog WNT5a in normal adipogenesis, we used the well-characterized mouse 3T3-L1 preadipocyte cell line. First we determined if Wnt5a and Wnt5b mRNA and protein in mouse was comparable to human, using the ClustalW analysis tool. Human Wnt5b encodes 2 different mRNAs which are $97 \%$ homologous, but encode the same protein, whereas for mouse only one mRNA is known. In both human and mouse, Wnt5a mRNA is about twice the size of Wnt5b. Human WNT5a mRNA shows resp. $38 \%$ and $39 \%$ homology to WNT5b NM_032642.2 and NM_030775.2 mRNA, and human WNT5a and WNT5b protein are $79.7 \%$ homologous. Mouse Wnt5a and $W n t 5 b$ are $42 \%$ homologous at the mRNA level and $77.7 \%$ at the protein level. Human WNT5a mRNA shows $70 \%$ mRNA homology and $98.6 \%$ on for the protein compared to mouse Wnt5a. Mouse Wnt5b shows resp. $73 \%$ and $75 \%$ mRNA homology with human WNT5b NM_032642.2 and NM_030775.2 and $94.1 \%$ for the protein. To study their role in adipogenesis, we first analyzed the normal expression pattern of Wnt5a and Wnt5b during 3T3-L1 differentiation. At two days post confluence, day 0 , differentiation was induced. RNA was isolated at day $0,1,2,4,7$ and 10 . As shown in figure 1 , the expression of both Wnt5a and Wnt5b was down-regulated during differentiation. Wnt5b

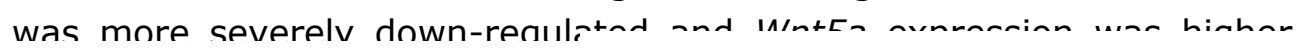
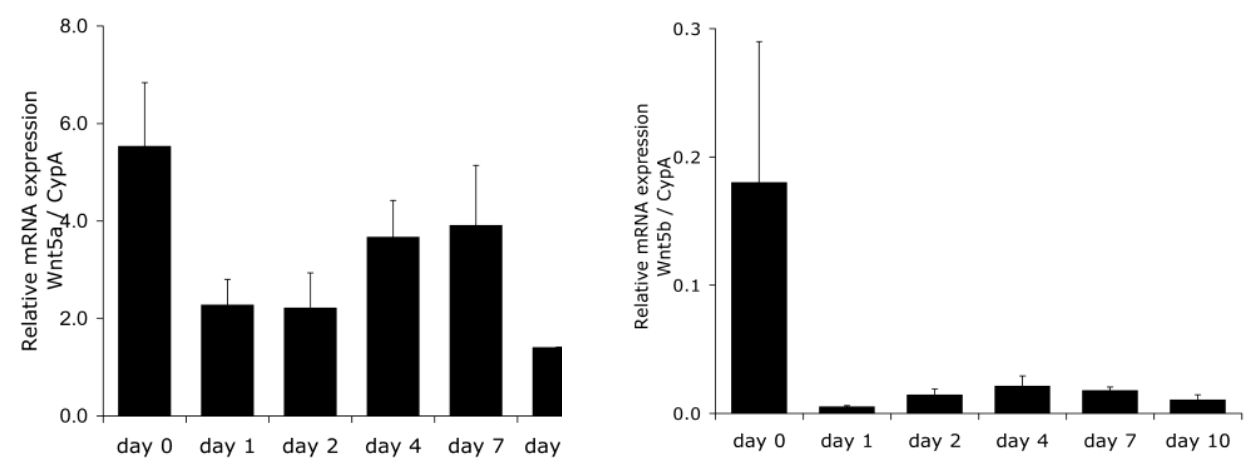

Fig. 1. Both Wnt5a and Wnt5b mRNA expression is down-regulated during differentiation of 3T3-L1 preadipocytes. Total RNA was isolated at indicated time-points, subjected to qPCR and expression was normalized with Cyclophilin A (CypA) expression. The bars indicate S.E. $(n=3)$. 
The effect of Wnt5b overexpression on adipocyte differentiation

To study the biological role of $W n t 5 b$ gene expression in adipogenesis, we analyzed the effect of Wnt5b overexpression during adipogenesis (experimental design Fig. 2A). In two independent experiments, mouse 3T3-L1 preadipocytes were transfected in duplo with pSPORT-Wnt5b overexpression construct or an empty pcDNA3.1 vector as transfection control. Two days post-confluence, the medium was replaced with differentiation medium and RNA was isolated at day 0, 2, 4, and day 10. $W n t 5 b$ overexpression resulted in increased expression of adipocyte specific transcription factor $a P 2$ (Fig. 2B). Additionally, the key transcription factor for adipogenesis, $P P A R Y$, was also significantly upregulated by $W n t 5 b$ at day 2,4 and 10 (Fig. 2C). The independent experiments yielded comparable results, although absolute values per culture differed. In figure $2 \mathrm{~B}$ and $2 \mathrm{C}$, the results of one representative experiment are shown.

The effect of Wnt5b overexpression on adipogenesis with stimulated $\beta$ catenin dependent Wnt signalling

The $\beta$-catenin dependent Wnt pathway was stimulated by incubation with Wnt3a-CM. which resulted in decreased adipogenesis as assessed by $a P 2$ and $P P A R Y$ expression (Fig. 2). Wnt5b overexpression resulted in significantly increased $a P 2$ and $P P A R Y$ expression. However, Wnt5b stimulated adipogenesis in Wnt3a-CM was still significantly less than in control medium without $W n t 5 b$ overexpression. Additionally, Wnt5a expression was reduced in cells incubated with Wnt3a-CM, but overexpression of Wnt5b significantly increased Wnt5a expression when incubated with Wnt3a-CM (Fig. 3).

To verify at protein level that Wnt3a-CM indeed stimulates $\beta$-catenin dependent Wnt signalling and the effect of $W n t 5 b$ overexpression, $\beta$ catenin quantification in cytoplasmatic and nuclear protein extracts was performed a day 0 . Wnt3a-CM resulted in a three-fold increase in nuclear $\beta$-catenin when compared to control medium. On protein level, at day $0,28 \% \beta$-catenin was observed in 3T3-L1 cells incubated in Wnt3a-CM and transfected with empty vector. In 3T3-L1 cells overexpressing $W n t 5 b$ and incubated with Wnt3a-CM, nuclear $\beta$-catenin was $17 \%$, while cells incubated with control medium, transfected with Wnt5b overexpression construct or empty vector, nuclear $\beta$-catenin was resp. $12 \%$ and $10 \%$ (Fig 4.). Wnt5b overexpression in cells incubated in 


\section{Chapter 5}

WNT3a-CM results in a $\sim 65 \%$ reduction in $\beta$-catenin translocation, but is still $\sim 35 \%$ higher than higher than in cells incubated with control medium.

a

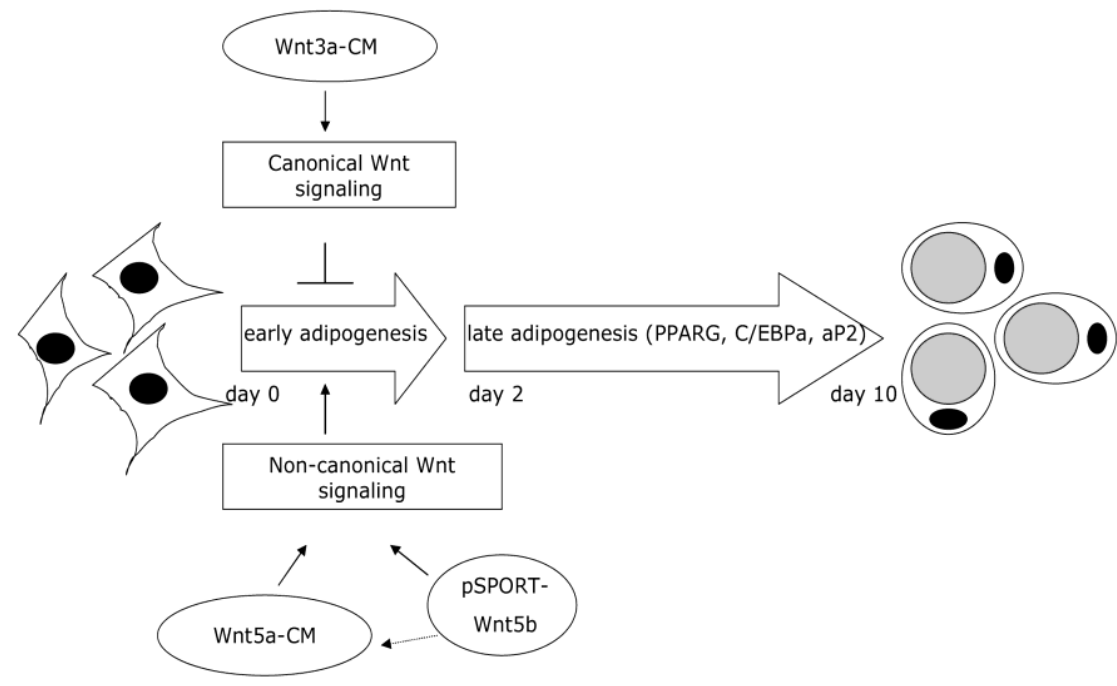

b

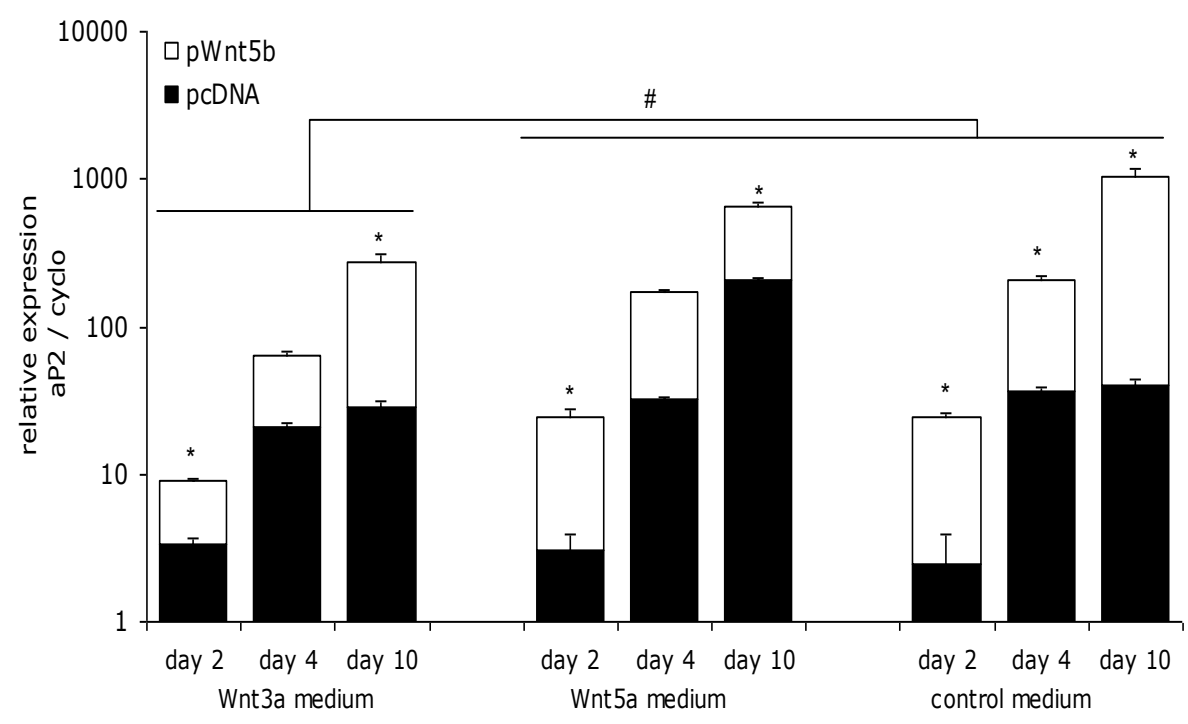


C

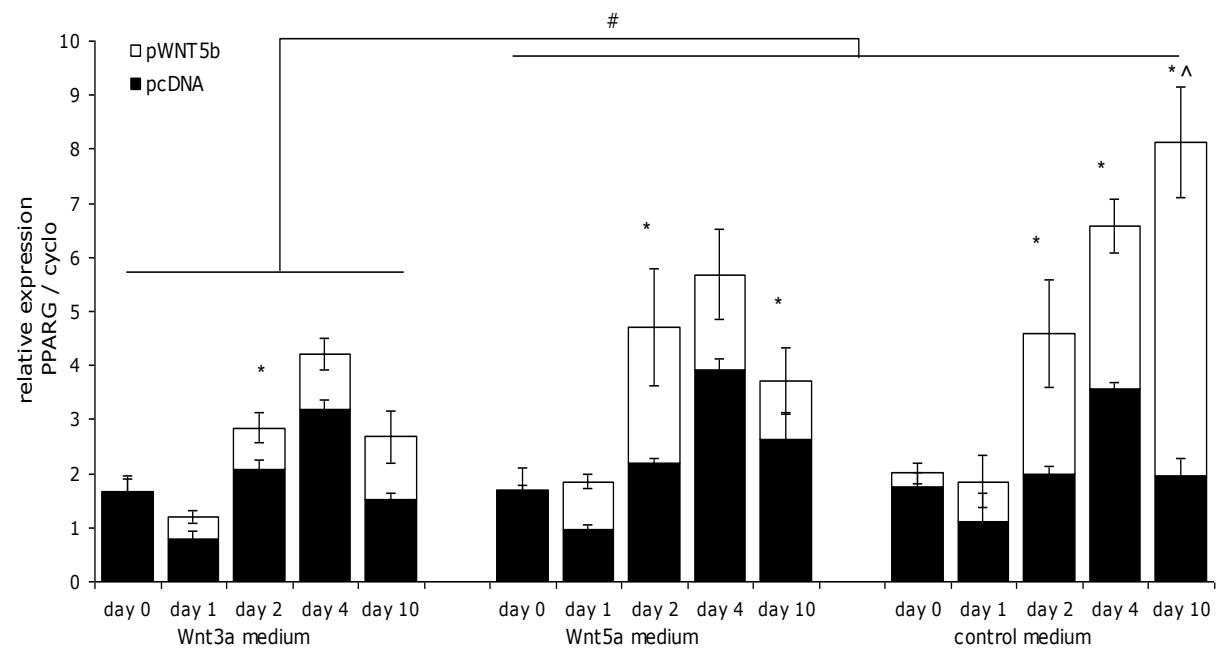

Fig. 2. Wnt5b overexpression enhances $a P 2$ and $P P A R Y$ expression during 3T3-L1 differentiation irrespective of stimulation of the $\beta$-catenin (in)dependent Wnt signaling pathway. 3T3-L1 cells were transfected with pSPORT-Wnt5b or pcDNA3.1 empty vector and differentiated in the presence of resp. 20\% L-cell control medium, 20\% Wnt5a-CM, or $20 \%$ Wnt3a-CM. Total RNA was isolated at indicated time-points, subjected to qPCR and expression was normalized with Cyclophilin A (CypA) expression. Error bars indicate S.D. of triplo RNA measurements for one representative experiment. A) Illustration of the experimental setup to study the effect of $W n t 5 b$ overexpression in combination with stimulation of the $\beta$-catenin independent Wnt signaling pathway by Wnt5a-CM or by stimulation of the $\beta$-catenin dependent Wnt signaling pathway with Wnt3a-CM. B) $a P 2$ mRNA expression of Wnt5b overexpressed 3T3-L1 cells compared to cells transfected with empty vector / time-point / medium. C) PPARY mRNA expression of Wnt5b overexpressed 3T3-L1 cells compared to cells transfected with empty vector / time-point / medium. * $\mathrm{p}<0.05$ Wnt5b overexpressed cells vs. empty vector transfected cells; \# $\mathrm{p}<0.05 \mathrm{Wnt3a}-\mathrm{CM}$ vs. Wnt5a-CM and control medium; $\wedge \mathrm{p}<0.05$ Wnt5b overexpressed cells in Wnt5a-CM vs. Wnt5b overexpressed cells in control medium.

The effect of Wnt5b overexpression on adipogenesis with stimulated $\beta$ catenin independent Wht signalling

Wnt5a conditioned medium (Wnt5a-CM) yields excessive availability of Wnt5a protein, which activates the $\beta$-catenin independent Wnt signalling pathway. We verified on protein level that Wnt5a-CM counteracts the $\beta$ catenin dependent Wnt-signalling in 3T3-L1 cells by quantifying nuclear and cytoplasmatic $\beta$-catenin from cells incubated with $20 \%$ Wnt3a-CM, or with both $20 \%$ Wnt3a-CM and $20 \%$ Wnt5a-CM. Addition of Wnt5a-CM resulted in a $60 \%$ reduction in nuclear $\beta$-catenin, indicating that Wnt5aCM inhibits $\beta$-catenin dependent Wnt signalling in 3T3-L1 cells (data not shown). 


\section{Chapter 5}

We explored if high availability of Wnt5a by addition of Wnt5a-CM had the same stimulatory effect on adipogenic potential as $W n t 5 b$ overexpression, and explored if a combination of Wnt5b overexpression and Wnt5a-CM (Fig 2) would increase adipogenic potential further. As shown in figure 2, Wnt5a-CM itself did not enhance $a P 2$ or $P P A R Y$ expression compared to control medium, but $W n t 5 b$ overexpression in combination with Wnt5a-CM resulted in significantly enhanced aP2 (Fig. 2B) and PPARY (Fig. 2C) expression at day 2 and 10. Wnt5a expression was reduced in Wnt5a-CM, which indicates compensation by paracrine signalling.

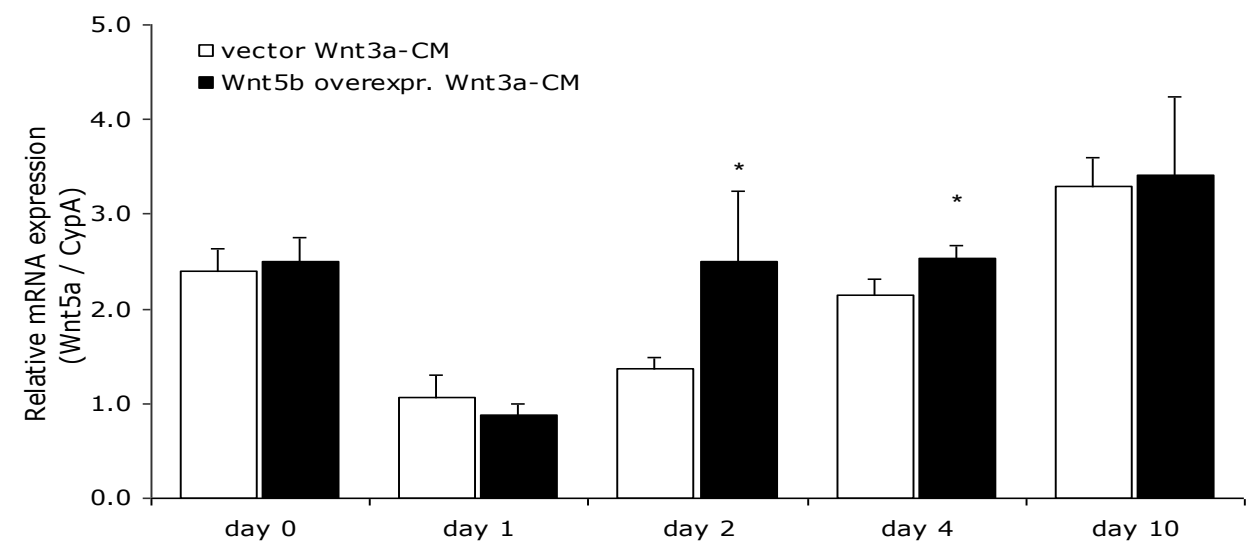

Fig. 3. $W n t 5 b$ overexpression enhances $W n t 5 a$ expression during 3T3-L1 differentiation with Wnt3a-CM. 3T3-L1 cells were transfected with pSPORT-Wnt5b or pcDNA3.1 empty vector and differentiated in the presence of $20 \%$ Wnt3a-CM. Total RNA was isolated at indicated time-points, subjected to qPCR and Wnt5a expression was normalized to the expression of Cyclophilin A (CypA). Error bars indicate S.E. $(n=2)$.

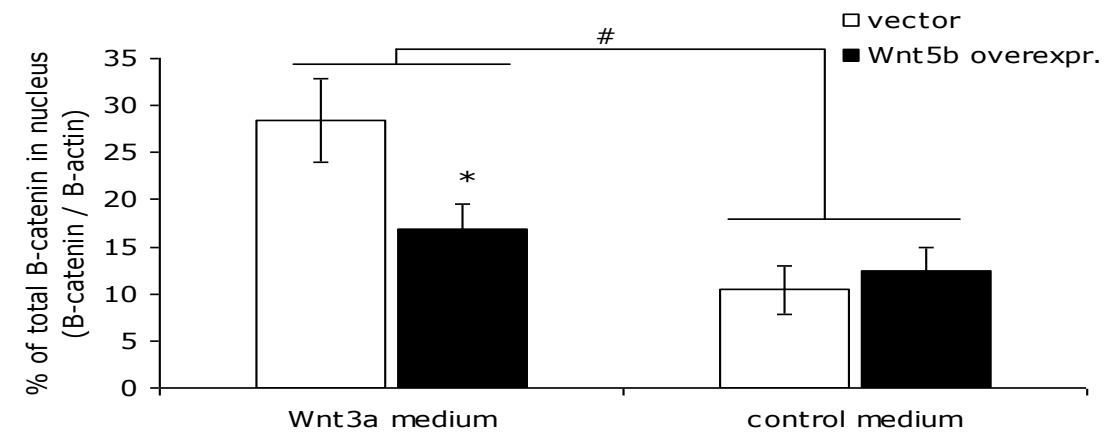

Fig. 4. Wnt5b overexpression reduces translocation of $\beta$-catenin to the nucleus. 3T3-L1 cells were transfected with pSPORT-Wnt5b or pCDNA3.1 empty vector and after 16 hours incubation with $20 \% \mathrm{Wnt} 3 \mathrm{a}-\mathrm{CM}$, nuclear and cytoplasmic proteins were isolated, loaded on SDS-PAGE gel, and incubated with anti- $\beta$-catenin. $\beta$-catenin level was normalized to the level of $\beta$-actin for loading. * $p<0.05 W n t 5 b$ overexpressed cells vs. vector transfected cells; \# $p<0.05$ Wnt3a-CM vs. control medium. Error bars indicate S.D. $(n=2)$. 


\section{Discussion}

Inhibition of $\beta$-catenin dependent Wnt signaling and stimulation of $\beta$ catenin independent Wnt signaling has been shown to be required for adipogenesis, and altered Wnt signaling has been observed in adipose tissue of T2DM subjects [2]. WNT5b was down-regulated in T2DM preadipocytes whereas the expression of its paralog WNT5a was unchanged (Van Tienen et al. submitted). The aim of our study was to determine the functional significance of this observation. First we determined the expression of both genes during adipogenesis. Analysis of mRNA and protein sequences showed that Wnt5a and Wnt5b are moderately conserved $( \pm 70 \%)$ at the mRNA level and highly conserved $( \pm 95 \%)$ at the protein level between mouse and human. Therefore, we used the mouse preadipocyte cell line 3T3-L1, which allows reproducible differentiation, to analyze expression of these genes during adipogenesis. Wnt5a was more abundantly expressed than Wnt5b, but both were down-regulated upon induction of differentiation. Overexpression of $W n t 5 b$ in control medium resulted in increased PPARY and $\mathrm{AP} 2$ expression, which are markers for adipogenesis (Fig. 2B and $2 \mathrm{C})$. In addition, stimulation of the $\beta$-catenin independent $\mathrm{Wnt}$ signaling pathway by enhancing Wnt5a protein availability (Wnt5a-CM) combined with $W n t 5 b$ overexpression did not enhance adipogenesis compared to control medium with $W n t 5 b$ overexpression. Stimulation of the $\beta$-catenin dependent Wnt signaling by Wnt3a has been shown to decrease adipogenic potential [5]. Inhibition of adipogenesis was also observed with Wnt3a-CM, but Wnt5b overexpression could partly overcome this inhibition and increase PPARY and $A P 2$ expression (Fig $2 \mathrm{~B}$ and $2 \mathrm{C}$ ). Still, aP2 expression was lower with Wnt3a-CM than control medium. On protein level, nuclear $\beta$-catenin translocation was partially inhibited $(65 \%)$ by $W n t 5 b$ overexpression. These data indicate that $W n t 5 b$ is a potent stimulator of adipogenesis, but is in our model not sufficient to completely overcome the inhibition of adipogenesis induced by Wnt3a$\mathrm{CM}$. Additionally, stimulation of the $\beta$-catenin dependent Wht signaling pathway with Wnt3a-CM resulted in reduced expression of $\beta$-catenin independent $W n t 5 a$, but $W n t 5 b$ overexpression was able to enhance Wnt5a expression, indicating that Wnt5b can enhance adipogenesis by stimulating Wnt5a expression and inhibiting $\beta$-catenin dependent Wnt signaling. In contrast to the stimulation by $W n t 5 b$ overexpression, 


\section{Chapter 5}

enhancing availability of Wnt5a protein by using Wnt5a-CM inhibited $\beta$ catenin translocation to the nucleus, but did not enhance adipogenesis or PPARY expression. Additionally, decreased Wnt5a expression was observed, indicating that paracrine signaling limits the Wnt5a availability. Our data suggests that $W n t 5 b$ is more crucial for stimulation of key regulators of adipogenesis than Wnt5a. The down-regulated expression of Wnt5a during differentiation is similar to that previously reported for Wnt5a by Nishizuka et al. [8], but the Wnt5b downregulation is contradictory to the up-regulation at day 2 observed by Kanazawa et al. [10]. Since both $W n t 5 a$ and $W n t 5 b$ function as inhibitors of $\beta$-catenin dependent Wnt signaling, and this inhibition of $\beta$ catenin dependent Wnt signaling at the initial phase of adipogenesis is required to allow adipogenesis, the observed expression pattern fits their function $[14,15]$. Additionally, Wnt5b can stimulate PPARY expression and subsequently enhance adipogenesis. However, PPARY is expressed from day 2 of differentiation, and not when Wnt5b has its highest expression, this stimulation is possibly mediated through upstream activators of $P P A R Y$, like $C / E B P \beta$ or $C / E B P \delta$, which are expressed in the same time-frame as $W n t 5 b$ [16].

The down-regulation of $\beta$-catenin independent $W n t 5 b$ in preadipocytes could contribute to decreased adipogenesis as observed in insulin resistance and type 2 diabetic subjects by reduced stimulation of PPARY [2]. However, the exact mechanism by which Wnt5b stimulates PPARY remains to be elucidated.

\section{Acknowledgements}

The research presented was funded by the Dutch Diabetes Foundation (DFN 2004.00.040). 


\section{References}

1. van Tienen, F.H., et al., Preadipocytes of Type 2 Diabetes subjects display an intrinsic gene expression profile of decreased differentiation capacity. International Journal of Obesity, 2010.

2. Yang, X., et al., Evidence of impaired adipogenesis in insulin resistance. Biochem Biophys Res Commun, 2004. 317(4): p. 1045-51.

3. Logan, C.Y. and R. Nusse, The Wht signaling pathway in development and disease. Annu Rev Cell Dev Biol, 2004. 20: p. 781-810.

4. Clevers, H., Wnt/beta-catenin signaling in development and disease. Cell, 2006. 127(3): p. 469-80.

5. Kennell, J.A. and O.A. MacDougald, Wnt signaling inhibits adipogenesis through beta-catenin-dependent and -independent mechanisms. J Biol Chem, 2005. 280(25): p. 24004-10.

6. Zhou, H., et al., Osteoblasts directly control lineage commitment of mesenchymal progenitor cells through Wnt signaling. J Biol Chem, 2008. 283(4): p. 1936-45.

7. Bennett, C.N., et al., Regulation of Wnt signaling during adipogenesis. J Biol Chem, 2002. 277(34): p. 30998-1004.

8. Nishizuka, M., et al., Wnt4 and Wnt5a promote adipocyte differentiation. FEBS Lett, 2008. 582(21-22): p. 3201-5.

9. Tseng, Y.H., et al., Prediction of preadipocyte differentiation by gene expression reveals role of insulin receptor substrates and necdin. Nat Cell Biol, 2005. 7(6): p. 601-11.

10. Kanazawa, A., et al., Association of the gene encoding wingless-type mammary tumor virus integration-site family member 5B (WNT5B) with type 2 diabetes. Am J Hum Genet, 2004. 75(5): p. 832-43.

11. Kanazawa, A., et al., Wnt5b partially inhibits canonical Wnt/beta-catenin signaling pathway and promotes adipogenesis in 3T3-L1 preadipocytes. Biochem Biophys Res Commun, 2005. 330(2): p. 505-10.

12. Larkin, M.A., et al., Clustal $W$ and Clustal $X$ version 2.0. Bioinformatics, 2007. 23(21): p. 2947-8.

13. Li, J., et al., Insig-1 "brakes" lipogenesis in adipocytes and inhibits differentiation of preadipocytes. Proc Natl Acad Sci U S A, 2003. 100(16): p. 9476-81.

14. Ross, S.E., et al., Inhibition of adipogenesis by Wnt signaling. Science, 2000. 289(5481): p. 950-3.

15. Topol, L., et al., Wnt-5a inhibits the canonical Wnt pathway by promoting GSK-3independent beta-catenin degradation. J Cell Biol, 2003. 162(5): p. 899-908.

16. Jazet, I.M., H. Pijl, and A.E. Meinders, Adipose tissue as an endocrine organ: impact on insulin resistance. Neth J Med, 2003. 61(6): p. 194-212. 



\section{Chapter}

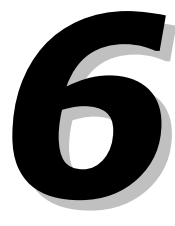

Prolonged Nrf1 overexpression triggers adipocyte inflammation and insulin resistance

F.H.J. van Tienen, P.J. Lindsey, C.J.H. van der Kallen, H.J.M. Smeets 


\section{Chapter 6}

\section{Abstract}

Adipose tissue is currently being recognized as an important endocrine organ, carrying defects in a number of metabolic diseases. Mitochondria play a key role in normal adipose tissue function and mitochondrial alterations can result in pathology, like lipodystrophy or type 2 diabetes. Although Pgc1a is regarded as the main regulator of mitochondrial function, downstream Nrf1 is the key regulator of mitochondrial biogenesis. Nrf1 is also involved in a wide range of other processes, including proliferation, innate immune response and apoptosis. To determine transcriptional targets of $\mathrm{Nrf1}$, 3T3-L1 preadipocytes were transfected with either pNrf1 or a control vector. Two days postconfluence, 3T3-L1 preadipocytes were allowed to differentiate. At day 8 of differentiation, Nrf1 overexpressing cells had an increased mtDNA copy number and reduced lipid content. This was not associated with an increased ATP production rate per cell. Using global gene expression analysis, we observed that Nrf1 overexpression stimulated cell proliferation, apoptosis, and cytokine expression. In addition, prolonged Nrf1 induced an adipokine expression profile of insulin resistant adipocytes. Nrf1 has a wide range of transcriptional targets, stimulators as well as inhibitors of adipose tissue functioning. Therefore, posttranscriptional regulation of Nrf1, or stimulating specific Nrf1 targets may be a more suitable approach for treating adipose tissue defects, instead of directly stimulating Nrf1 expression. In addition, our results show that despite promising short-term effects, long-term effects can drastically differ. 


\section{Introduction}

The incidence of obesity and obesity-associated diseases, like cardiovascular diseases, hypertension, dyslipidemia and Type 2 Diabetes Mellitus (T2DM) is rapidly increasing throughout the world [1]. Obesity and T2DM are currently being considered world-wide epidemics [2]. Adipose tissue is together with skeletal muscle, a key organ in development of both obesity and T2DM. Obesity is characterized by excessive caloric intake that requires increased adipocyte proliferation and adipocyte enlargement. Prolonged excessive adipocyte overload can lead to adipocyte dysfunction, which is characterized by increased macrophage content, inflammation, lipolysis, altered adipokine secretion profile, insulin resistance and decreased adipocyte differentiation capacity [3].

Central in normal lipid metabolism are the mitochondria. Mitochondria are responsible for the majority of ATP production in a cell and accomplish a number of important cellular processes, like regulating the balance between fatty acid synthesis, $\beta$-oxidation and lipolysis. These processes are impaired in both skeletal muscle and adipose tissue from T2DM subjects [4-6]. The relation between mitochondrial aberrations and abnormal lipid metabolism is also exemplified by observations that m.8344A $>$ G, m.8363G $>A$ and m.3271T $>C$ mutation carriers, and persons having a mtDNA depletion due to anti-retroviral therapy, may develop lipodystrophy and T2DM as part of the clinical spectrum $[7,8]$. In addition, decreased mitochondrial density and activity, as well as decreased expression of key regulators of mitochondrial biogenesis have been observed in muscle and adipose tissue of T2DM patients [5, 6, 9, 10]. One of those regulators is peroxisome-proliferator activated receptor Y coactivator $1 \mathrm{a}(\operatorname{PgC1a})$. Pgc1a is a transcriptional co-activator that is involved in regulation of a number of transcription factors involved in energy metabolism, e.g. PPARY, Nrf1, ERRa, and CREB. As a result, Pgc1a provides a direct link between external physiological stimuli and the regulation of mitochondrial biogenesis [11]. Thiazolidinediones (TZDs), e.g. pioglitazone and rosiglitazone, are antidiabetic agents that activate the adipogenic transcription factor PPARY, resulting in improved adipose tissue mitochondrial function, insulin sensitivity, and adipokine expression [9, 12-14]. Like pioglitazone, two 


\section{Chapter 6}

days of Nrf1 overexpression results in increased adiponectin secretion, which regulates energy homeostasis and glucose and lipid metabolism in adipocytes [15].

Although Nrf1 is mainly regarded as a Pgc1a target, which controls Tfam expression and mitochondrial biogenesis, altered Pgc1a expression does not necessarily influence Nrf1. For example, inactivity resulted in decreased Pgc1a expression whereas Nrf1 expression was increased [16], and Nrf1 is not influenced by Sirt1, which is an upstream activator of Pgc1a [17]. Nrf1 encodes a phosphorylated nuclear protein with a bZIP binding domain and plays, besides stimulating mitochondrial biogenesis, a crucial role in multiple other processes. Immunoprecipitation assays have shown that Nrf1 targets $\sim 700$ promoters of genes involved in a number of functions, e.g. DNA replication and repair, cell proliferation, migration and apoptosis [18]. Taken together, current data indicates that Nrf1 is more than a Pgc1a target and mitochondrial dysfunction in adipose tissue and reduced Nrf1 are key aspects of T2DM development. In this study we aim to identify the genes that are directly regulated by Nrf1 in adipocytes, which may help identifying new more specific downstream therapeutic targets for T2DM, or other diseases associated with mitochondrial dysfunction.

\section{Materials and Methods}

\section{Cell culture}

Mouse 3T3-L1 preadipocytes (ATCC) were grown in Dulbecco's modified Eagle's medium (DMEM) (Gibco) containing 10\% FCS (Gibco) and penicillin/streptavidin (Gibco). As shown in figure 1, 3T3-L1 preadipocytes were transfected at $70 \%$ confluence with pSPORT-Nrf1 (Openbiosystems) or pEGFP control vector with Fugene HD transfection reagents (Roche) according to the manufacturers' protocol. Transfection was repeated at day 4 of differentiation. Differentiation was induced in two-days post-confluence by changing medium with DMEM containing $0.5 \mathrm{mM}$ 3-isobutyl-1-methylxantine (IBMX) (Sigma), $10 \mu \mathrm{g} / \mathrm{ml}$ insulin (Sigma) and $1 \mu \mathrm{M}$ dexamethasone (Sigma). After two days, medium was replaced with DMEM containing $10 \%$ FCS and $10 \mu \mathrm{g} / \mathrm{ml}$ insulin, and changed every two days till day 8. 


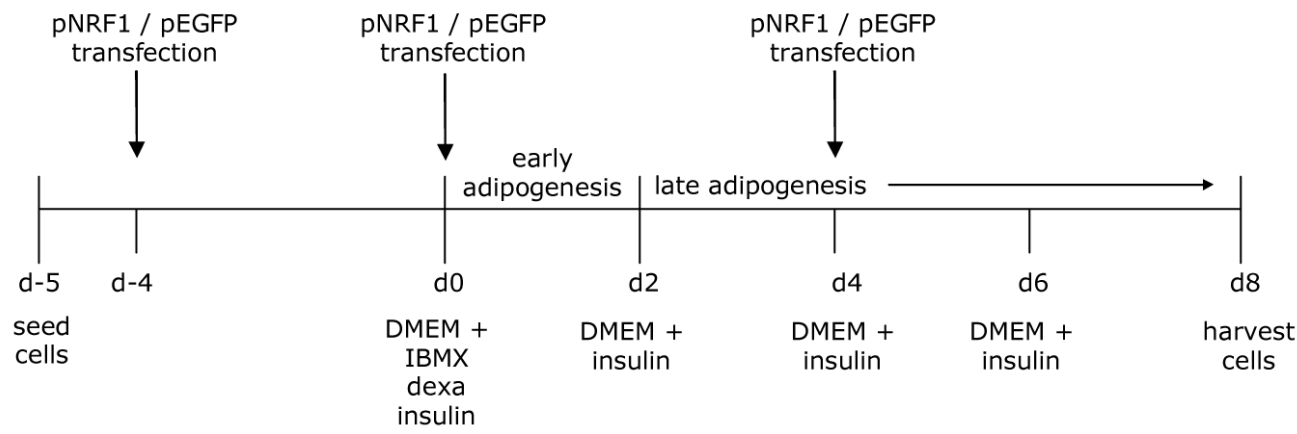

Fig. 1. Experimental setup

3T3-L1 cells were seeded at day -5 and $70 \%$ confluent at d-4 when 3T3-L1 cells were transfected with either PSPORT-Nrf1 overexpression construct or control pEGFP vector. Two-days post-confluence, at day 0 , transfection was repeated and differentiation mix was added. Cells were differentiated for 8 days with refreshing medium+insulin every other day. IBMX: 3-isobutyl-1-methylxanthine, dexa: dexamethasone.

\section{mtDNA copy number determination}

DNA was isolated using the Qiagen DNA isolation kit according to the manufacturers' protocol (Qiagen, Hilden, Germany). The mtDNA content was determined by comparing the ratio of mtDNA (ND1) to nDNA (18S rRNA) in triplo by real-time quantitative PCR. DNA was amplified in a $12.5 \mu$ l reaction containing $5 \mathrm{ng}$ DNA, $1.25 \mathrm{pmol}$ of forward and reverse primer, and ABI SYBR Green PCR master mix (Applied Biosystems, Carlsbad, CA, USA). The cycling conditions were: an initial step for $2^{\prime}$ at $50^{\circ} \mathrm{C}$, activation of the Hot Goldstar enzyme at $95^{\circ} \mathrm{C}$ for $10^{\prime}, 40$ cycles of $15^{\prime \prime}$ at $95^{\circ} \mathrm{C}$ followed by $1^{\prime}$ at $60^{\circ} \mathrm{C}$.

Gene expression analysis

RNA isolation and DNAse treatment were performed with the high pure RNA isolation kit (Roche) according to the manufacturers' protocol. RNA quantity and purity were determined spectrophotometrically using the Nanodrop ND-1000 (Nanodrop Technologies, Wilmington - DE, USA) and RNA integrity was assessed by determining the RNA 28S/18S ratio using the Bioanalyzer 2100 (Agilent Technologies, Santa Clara - CA, USA). Mouse adipocyte RNA spiked with four bacterial RNA transcripts was reverse transcribed into CDNA and amplified in a two-round amplification reaction according to the manufacturer's protocol (Affymetrix, Santa Clara - CA, USA). A mixture of cDNA and added hybridization controls was hybridized on Affymetrix Mouse Genome 430 2.0 arrays, followed by staining and washing steps in the GeneChip 


\section{Chapter 6}

fluidics station 400 and were scanned using the GeneChip scanner 3000 (Affymetrix.

Detection of over-represented transcription factor binding sites

The online tool oPOSSUM V2.0 was used to identify over-represented transcription factor binding sites in the promoters of differentially expressed genes [19]. All genes with $>20 \%$ up- and down-regulated expression in NRF1-overexpressing 3T3-L1 cells at day 8 of differentiation were uploaded in oPossum. Settings were as followed: Taxonomic supergroup: vertebrates, top 10 level of conserved regions (min. conservation $>70 \%$ ), matrix match threshold $80 \%$, 2000 bp upand downstream sequence, $Z$-score $\geq 10$.

\section{Statistical analysis}

Statistical analysis was performed using the freely available program $\mathrm{R}$ [20] and the publicly available library 'growth' [21]. The chip description file (CDF) used for the analysis was an update [22, 23] based on Ensembl (version 10). This resulted in the analysis of 15768 genetranscripts. All genes were analyzed using a Gaussian linear regression ( $\mathrm{N}\left(\mu, \sigma^{2}\right)$, where $\mu$ is the mean and $\sigma^{2}$ is the variance, including the chip's log mean intensity and the best hybridization spike. The inference criterion used for comparing the models is their ability to predict the observed data, i.e. models are compared directly through their minimized minus log-likelihood. When the numbers of parameters in models differ, they are penalized by adding the number of estimated parameters, a form of the Akaike information criterion (AIC) [24]. The transcript under consideration was found to be differentially expressed, if the AIC decreased compared to the model not containing the group effect, the confidence interval did not include a fold change of 1 , there was at least a $20 \%$ fold change increase or decrease, and each group had a mean signal intensity $>200$ or $>300$ in one group. Visualization and analysis of microarray data on biological pathways was performed using the Gene Map Annotator and Pathway Profiler (GenMAPP, version 2.0) [25]. Gene database Mm-std_20070817 and MAPPs version Mm_contributed_20080619 were used. Significantly changed pathways were identified using the MAPPFinder program, which expresses a " $\mathrm{z}$ score" for each pathway using Fisher's exact test. z-scores $>1.96$ and permute $P \leq 0.05$ were considered to be significant [26]. In addition, 
gene ontology (GO) based analysis of biological processes of level 5 were analyzed in DAVID [27].

Quantitative real-time $P C R$

CDNA was generated from $1 \mu \mathrm{g}$ RNA in a standard reverse transcriptase reaction using M-MulV reverse transcriptase (Finnzymes, Espoo, Fi). Primers were designed using Primer Express ${ }^{\circledR}$ software version 3.0 (Applied Biosystems; for primer sequences, see Electronic Supplementary Material (ESM 4). All ESM can be found at http://www.personeel.unimaas.nl/florence-vantienen/, also indicated by reference [28]. Quantification of transcripts was carried out using the ABI 7900 HT Real-Time PCR detection system using Eurogentec qPCR Mastermix Plus for SYBR Green $\AA$ I (Eurogentec, Seraing, B). The cycling conditions were: an initial step for $2^{\prime}$ at $50^{\circ} \mathrm{C}$, activation of the Hot Goldstar enzyme at $95^{\circ} \mathrm{C}$ for $10^{\prime}, 40$ cycles of $15^{\prime \prime}$ at $95^{\circ} \mathrm{C}$ followed by $1^{\prime}$ at $60^{\circ} \mathrm{C}$. The mRNA levels of each gene were normalized to those of the housekeeping gene encoding the Cyclophilin. Primer sequences for the analyzed genes can be found in the Electronic Supplementary Material (ESM 4 [28]).

ATP production and Caspase 3/7 assays

ATP production in 3T3-L1 cells transfected with PSPORT-Nrf1 or pEGFP (control) was measured at day 8 using the CellTiter-Glo luminescent Cell viability assay (Promega, Madison, WI, USA) according manufacturer's protocol. ATP production was corrected for cell density by quantification of dsDNA determined with the Quant-iT PicoGreen dsDNA kit (Invitrogen, Carlsbad, CA, USA). Caspase 3/7 activity was measured at day 8 , after trypsinization, cell were counted and an equal amount of cells per well were used for quantification of caspase 3/7 activity using the Caspase-Glo $₫ 3 / 7$ assay (Promega) according to manufacturer's protocol.

\section{Crystal violet staining}

Cell numbers were assessed by the crystal violet assay. Cells were fixed for 1 hour in $3.7 \%$ paraformaldehyde in PBS. They were stained for 30 min with a $0.1 \%$ solution of crystal violet, and destained 5 times in demineralised water. The dye was released with $10 \%$ acetic acid at RT for $30 \mathrm{~min}$, and the $A_{590}$ signal was then read using the Multiskan Spectrum microplate spectrophotometer (Thermo Fisher Scientific). 


\section{Chapter 6}

\section{Electron microscopy}

Cells were fixed overnight in $2.5 \%$ glutaraldehyde (Merck, Darmstadt, D), post-fixed in $1 \%$ osmium tetroxide solution, dehydrated, and embedded in epoxy resin. Semi-thin $(1 \mu \mathrm{m})$ serial sections were stained with toluidine blue. Ultra-thin sections ( 70 to $90 \mathrm{~nm}$ ) were mounted on Formvar-coated 75 mesh copper grids (1595 E, Merck, Amsterdam, NL), and counterstained with uranyl acetate and lead citrate before analysis on a Philips CM100 transmission electron microscope (Philips, Eindhoven, NL). Quantitative analysis of mitochondrial size was carried out using freely available Image J software (version $1.42 q$ ).

\section{Results}

Mouse 3T3-L1 preadipocytes were transfected with either a vector containing Nrf1 or a control vector without Nrf1 (pEGFP), and were allowed to differentiate for eight days (Fig. 1). Mitochondrial DNA copy number and gene expression of Nrf1 and adipogenic regulators were determined at several time points after transfection and during differentiation to test if the transfection showed the expected result. On day 8 of differentiation, analysis of mitochondrial function, density and morphology were analyzed together with global gene expression levels to determine transcriptional targets of Nrf1.

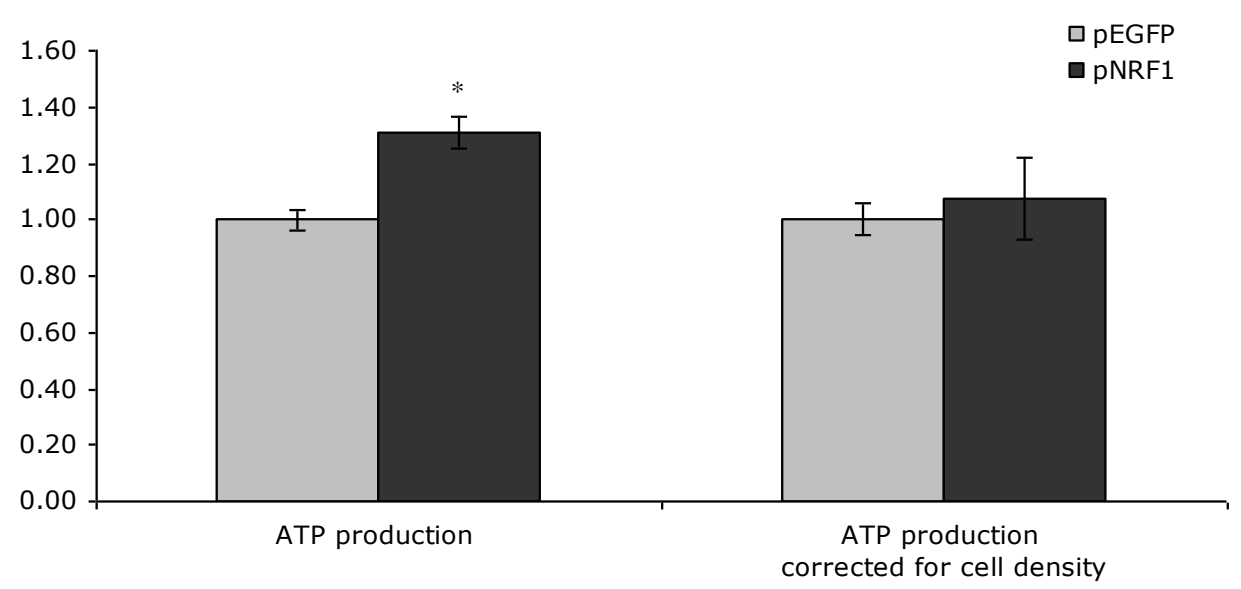

Fig. 2. ATP production in 3T3-L1 adipocytes at day 8 of differentiation.

3T3-L1 cells were seeded at the same density, transfected at 70\% confluence with pNrf1 or pEGFP and differentiation was induced 2 -days post-confluence. $n=4, * p<0.05$. 
Effect of Nrf1 overexpression on mitochondrial parameters in differentiating preadipocytes

MtDNA copy number was quantified by the ratio between mitochondrial encoded ND1 and nuclear encoded $18 S$ rRNA. The mitochondrial DNA copy number in 3T3-L1 adipocytes was significantly increased $(P=0.01$; $\mathrm{n}=4)$ in pNrf1 overexpressing cells $(1.53 \pm 0.3)$ compared with control pEGFP transfected cells. To identify if the mtDNA was continuously increased during differentiation, we analyzed the mtDNA content at day $0,3,6$ and 8 , and observed only a significantly higher mtDNA content in pNRF1-transfected cells at day 6 and day 8, but not earlier (data not shown). To evaluate if $N r f 1$ overexpression resulted in increased ATP production, we measured ATP production at day 8 of differentiation in Nrf1 overexpressing and control pEGFP-transfected 3T3-L1 adipocytes. As shown in figure 2, increased ATP production was observed in Nrf1overexpressing cells. However, after correcting for number of cells, no difference was observed in ATP production. Since cells were seeded at same density, increased cell number in Nrf1-overexpressing cells suggests increased proliferation due to Nrf1 overexpression. Crystal violet staining at day 0 and day 8 showed indeed a $13 \%(P=0.07)$ and $36 \%(P<0.01)$ increased cell number during differentiation in Nrf1 transfected wells. Furthermore, electron microscopic analysis of mitochondrial morphology showed that Nrf1-overexpressing cells had enlarged (Fig. 5,6) and more electron dense mitochondria than pEGFPtransfected cells at day 8 of differentiation (Fig. 5).

Differentially expressed genes due to Nrf1 overexpression in preadipocytes

The effect of Nrf1 overexpression on gene expression was assessed by comparing four replicates of 3T3-L1 cells transfected with pNrf1 overexpression construct with four replicates transfected with pEGFP as controls on day 8 of differentiation. Gene expression analysis was performed using Affymetrix Mouse Genome 4302.0 arrays, and after correction for probe set annotation, 15,768 transcripts were analyzed. Using normal regression modeling, a total of 1,926 transcripts were found differentially expressed between control cells and Nrf1 overexpressing cells with a fold-change of minimal $20 \%$. From these 1,926 transcripts, 920 transcripts were increased, and 1,006 transcripts were decreased in Nrf1 overexpressing 3T3-L1 cells. The majority of the 


\section{Chapter 6}

differentially expressed genes had a fold change of less than two-fold up or down. From the 1,006 transcripts with decreased expression, 51 $(5.1 \%)$ had a fold change $<0.5$, and the most extreme down-regulated probe had a fold change of 0.22 . Of the 920 up-regulated transcripts, 76 transcripts $(8.3 \%)$ were more than two times higher. A few of the increased genes had an extreme fold change with a maximum of 57 fold. The distribution of the fold changes is depicted in electronic supplementary material (ESM) 1 [28], and a list of the 10 most severely up- and down-regulated genes is presented in Table 1.

Table 1. Top 10 most up- and down-regulated transcripts in Nrf1-overexpresing cells

\begin{tabular}{|c|c|c|}
\hline Name & Symbol & $\begin{array}{l}\text { Fold change } \mathrm{Nrf1-} \\
\text { overexpressing cells }\end{array}$ \\
\hline \multicolumn{3}{|l|}{ Down-regulated in NRF1 overexpressing cells } \\
\hline Adiponectin & Adipoq & $0.22(0.11-0.43)$ \\
\hline $\mathrm{Na}+/ \mathrm{K}+$ transporting ATPase subunit beta- 1 & Atp1b1 & $0.22(0.21-0.24)$ \\
\hline Leucine-rich repeat-containing protein 17 Precursor & Lrrc17 & $0.24(0.21-0.28)$ \\
\hline Type-2 angiotensin II receptor & Agtr2 & $0.25(0.24-0.27)$ \\
\hline Matrix Gla protein & Mgp & $0.26(0.25-0.27)$ \\
\hline Fatty acid-binding protein 4 & Fabp4 & $0.27(0.24-0.32)$ \\
\hline ATP-binding cassette sub-family A member 8-A & Abca8a & $0.28(0.27-0.29)$ \\
\hline Dermatopontin & $D p t$ & $0.31(0.29-0.33)$ \\
\hline DNA-binding protein inhibitor ID-2 & $I d 2$ & $0.31(0.29-0.33)$ \\
\hline small proline-rich protein $1 \mathrm{~A}$ & Sprr1a & $0.31(0.30-0.32)$ \\
\hline \multicolumn{3}{|l|}{ Up-regulated in NRF1 overexpressing cells } \\
\hline Cd74 antigen & $C d 74$ & $5.06(4.22-6.08)$ \\
\hline Chitinase 3 -like 1 & Chi3/1 & $5.17(4.96-5.39)$ \\
\hline Chemokine (C-X-C motif) ligand 11 & Cxcl11 & $5.34(5.15-5.53)$ \\
\hline Complement factor B & $C f b$ & $6.99(6.67-7.33)$ \\
\hline Prolactin family 2 , subfamily $c$, member 4 & Prl2c4 & $7.20(6.13-8.47)$ \\
\hline Lipopolysaccharide binding protein & $L b p$ & $10.4(10.2-10.7)$ \\
\hline Chemokine ( $\mathrm{C}-\mathrm{X}-\mathrm{C}$ motif) ligand 5 & Cxcl5 & $10.5(9.22-12.0)$ \\
\hline Haptoglobin & $H p$ & $21.8(20.8-22.8)$ \\
\hline Serum amyloid $A 3$ protein & Saa3 & $35.4(33.0-37.8)$ \\
\hline Lipocalin 2 & $\operatorname{Lcn} 2$ & $56.8(53.0-60.8)$ \\
\hline
\end{tabular}

Data are means \pm confidence interval (CI)

We used a process-based approach to identify differentially expressed pathways in adipocytes due to overexpression of Nrf1. The 1,926 differently expressed genes that could be annotated were used for gene ontology based analysis using the GenMAPP/MAPPFinder program, which performs global analysis of expression data in the context of hundreds of 
pathway MAPPs and thousands of Gene Ontology (GO) terms, calculates the cumulative total of genes changed for a local MAPP or term, and provides a statistical z-score to assess significance (Table 2, ESM 2). Eight pathways (Table 4) were found significantly changed with MAPPFinder analysis with a $z$-score $>1.96$ and permute $P \leq 0.05$. In addition, ten $\mathrm{GO}$ biological processes at level 5 were found significantly changed $(\mathrm{P} \leq 0.05)$ in pNrf1 transfected cells, and comprised cell-cycle regulation, inflammation and apoptosis related genes, as seen in the Mappfinder analysis (ESM 3 [28]).

Table 2. $\mathrm{qPCR}$ validation of microarray data

\begin{tabular}{lccc}
\hline Gene & Symbol & $\begin{array}{c}\text { Fold change } \\
\text { microarray }( \pm \mathrm{CI})\end{array}$ & $\begin{array}{c}\text { Fold change } \\
\text { qPCR }( \pm \mathrm{CI})\end{array}$ \\
\hline Fatty acid-binding protein 4 & Fabp4 & $0.27(0.24-0.32)$ & $0.31(0.26-0.39)$ \\
Superoxide dismutase 2 & Sod2 & $3.35(3.19-3.52)$ & $1.82(1.65-2.00)$ \\
Ceruloplasmin & $C p$ & $4.22(4.12-4.34)$ & $4.00(3.36-4.75)$ \\
Haptoglobin & $H p$ & $21.8(20.8-22.8)$ & $215(179-257)$ \\
Serum amyloid A-3 protein & Saa3 & $35.4(33.0-37.8)$ & $369(312-437)$ \\
Adiponectin & Adipoq & $0.22(0.11-0.43)$ & $0.22(0.18-0.26)$ \\
Matrix Gla protein & Mgp & $0.26(0.25-0.27)$ & $0.31(0.27-0.35)$ \\
\hline
\end{tabular}

Data are means \pm confidence interval $(\mathrm{CI})$

Table 3. Overrepresented transcription-factor bindingssites in Nrf1-induced genes

\begin{tabular}{lccc}
\hline Transcription factor (TF) & TF Class & nr. target gene hits & Z score \\
\hline $\begin{array}{l}\text { up-regulated genes } \\
\text { Hepatic leukemia factor (HLF) }\end{array}$ & bZIP & 203 & 12.3 \\
down-regulated genes & & & \\
Serum response factor (SRF) & MADS & 57 & 20.1 \\
Sex-determining Region Y (SRY) & HMG & 579 & 12.1 \\
Forkhead box q1 (Foxq1) & FORKHEAD & 273 & 11.6 \\
Aryl hydrocarbon receptor nuclear & bHLH & 625 & 11.3 \\
translocator / receptor (Arnt-Ahr) & & & \\
SRY-related HMG-box (Sox5) & HMG & 586 & 11.1 \\
\hline Z
\end{tabular}

$Z$ score $>10$ were considered to be significant

Detection of potential Nrf1 overrepresented transcription factor binding sites

Nrf1 contains a bZIP domain and oPOSSUM analysis of the up-regulated genes in Nrf1 overexpressed cells at day 8 of adipogenesis showed that the hepatic leukemia factor (HLF), which belongs to the bZIP class of transcription factors, was solely enriched (Table 3). Subsequent GO biological process analysis of all 203 up-regulated genes containing the 


\section{Chapter 6}

bZIP domain showed they were involved in proliferation and cell-cycle regulation, apoptosis and chemokine/cytokine signaling (ESM 5 [28]). Within the group of down-regulated genes, a more heterogeneous group of five transcription factor binding sites were overrepresented (Table 3 ).

Table 4. Identification of significantly changed pathways by MAPPFinder analysis

\begin{tabular}{lcccc}
\hline MAPP Name & \multicolumn{5}{c}{$\%$} \\
Mm_Adipogenesis & 29 & 92 & 4.28 & 0.00 \\
Mm_Apoptosis & 28 & 96 & 3.09 & 0.00 \\
Mm_Id_NetPath_5 & 30 & 94 & 2.89 & 0.01 \\
Mm_Delta-Notch_NetPath_3 & 28 & 87 & 2.88 & 0.01 \\
Mm_Hypertrophy_model & 38 & 80 & 2.48 & 0.03 \\
Mm_Matrix_Metalloproteinases & 33 & 96 & 2.48 & 0.02 \\
Mm_TGF_Beta_Signaling_Pathway & 27 & 94 & 2.30 & 0.03 \\
Mm_Fas_Pathway_and_Stress_Induction_ & 29 & 92 & 2.21 & 0.04 \\
of_HSP_Regulation & & & &
\end{tabular}

$Z$-score values $>1.96$ and Permute $\mathrm{P} \leq 0.05$ were considered to be significant
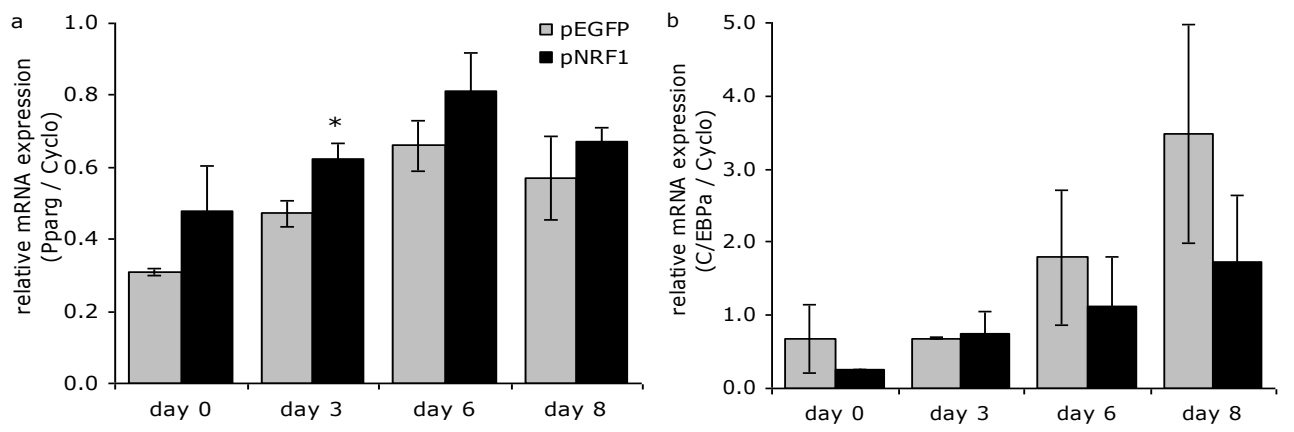

Fig. 3. Ppary and C/EBPa expression during 3T3-L1 differentiation

3T3-L1 cells were transfected with PSPORT-NRF1 or pEGFP empty vector and differentiated. Total RNA was isolated at indicated time-points, subjected to qPCR and expression of Ppary (a) and C/EBPa (b) was normalized to the expression of Cyclophilin A $($ СурA). Values are mean \pm S.D. of three determinations, $* p<0.05$ when compared to pEGFP transfected cells at the same time-point.

\section{Expression analysis during adipogenesis}

A decreased adipogenic capacity of Nrf1-transfected cells could also explain our results, to test this, we transfected 3T3-L1 cells with either pNrf1 or control pEGFP, and harvested at different time-points during adipogenesis. In this way we could determine if differential expression at day 8 of adipogenesis was directly caused by Nrf1 overexpression or was a secondary result. At day 0 , day 3 , day 6 and day 8 , qPCR analysis 

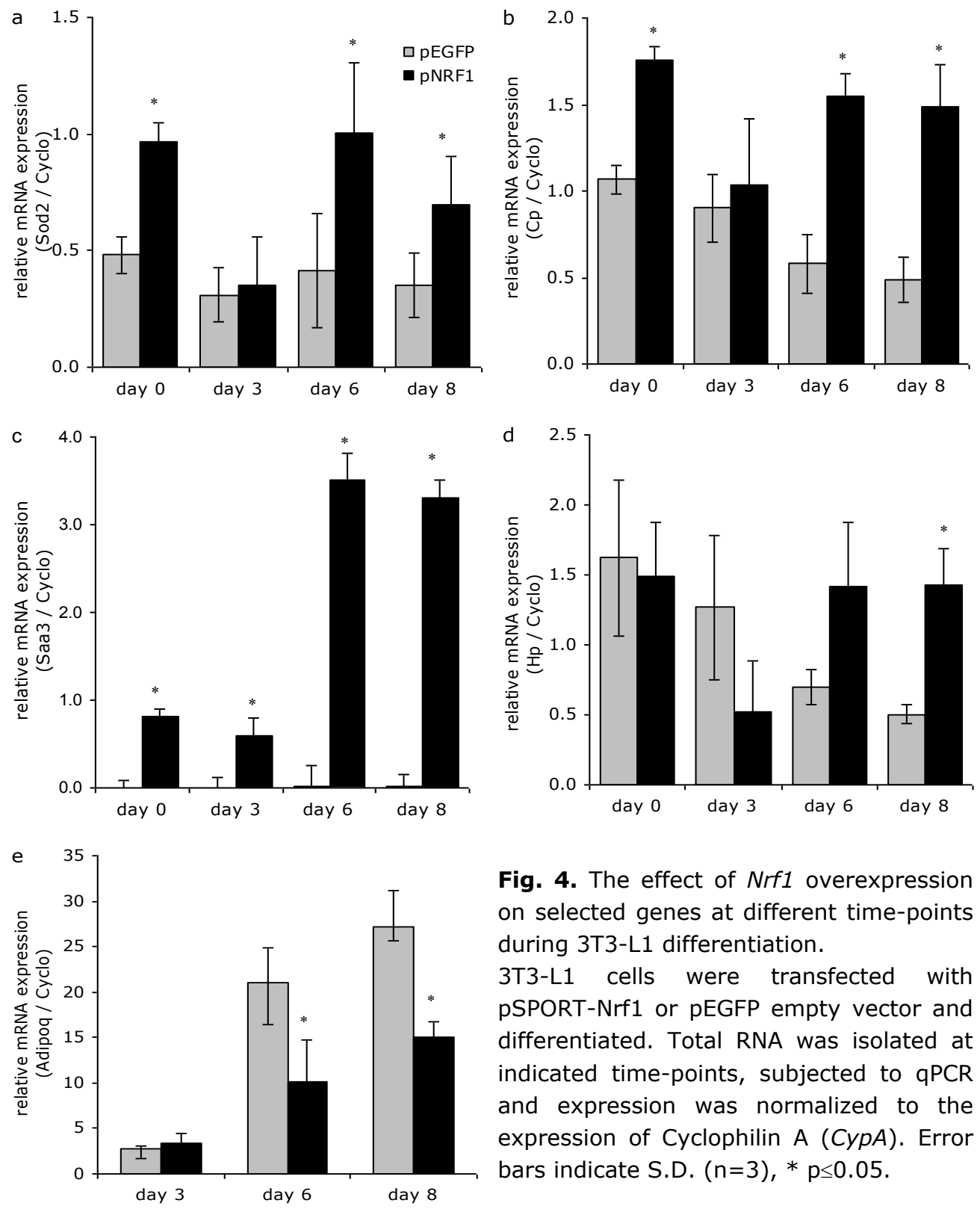

Fig. 4. The effect of Nrf1 overexpression on selected genes at different time-points during 3T3-L1 differentiation.

3T3-L1 cells were transfected with pSPORT-Nrf1 or pEGFP empty vector and differentiated. Total RNA was isolated at indicated time-points, subjected to qPCR and expression was normalized to the expression of Cyclophilin A (CypA). Error bars indicate S.D. $(n=3), * p \leq 0.05$. 


\section{Chapter 6}

was performed of the key regulators of adipogenesis, Ppary and C/EBPa. As shown in figure 3, expression of these two key regulators was not significantly different during adipogenesis. Moreover, Ppary expression was slightly increased in Nrf1 overexpressing cells at all time-points, but this was only significant at day 3 of differentiation. In addition, expression of superoxide dismutase 2 (Sod2), Ceruloplasmin $(C p)$ and Serum amyloid A-3 (Saa3) were higher in Nrf1-overexpressing cells at minimal three of the four time-points, while adiponectin and fatty acid binding protein 4 (Fabp4) were only increased at day 6 and/or 8 of differentiation (Fig. 4).

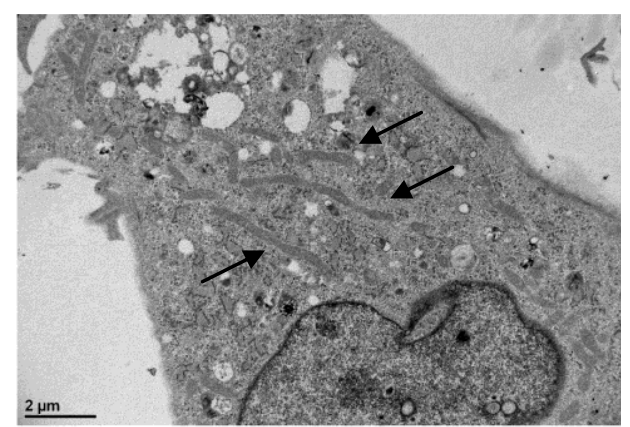

a

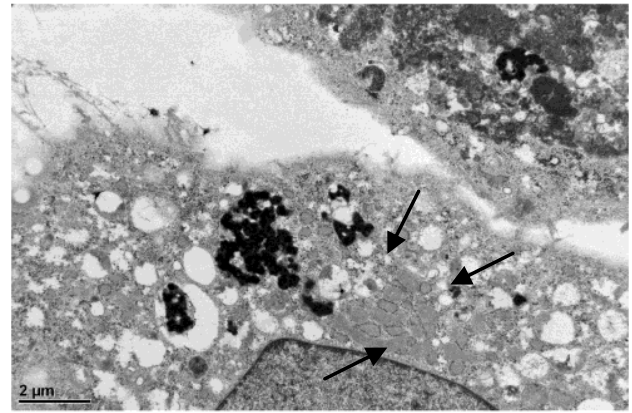

b

Fig. 5. Representative electron microscopy pictures of pNrf1 transfected (a) and control pEGFP transfected (b) 3T3-L1 cells at day 8 of differentiation. Mitochondria are indicated by arrows (not all mitochondria are indicated). Nrf1-transfected cells (a) have more electron dense and larger mitochondria than pEGFP transfected cells (b).

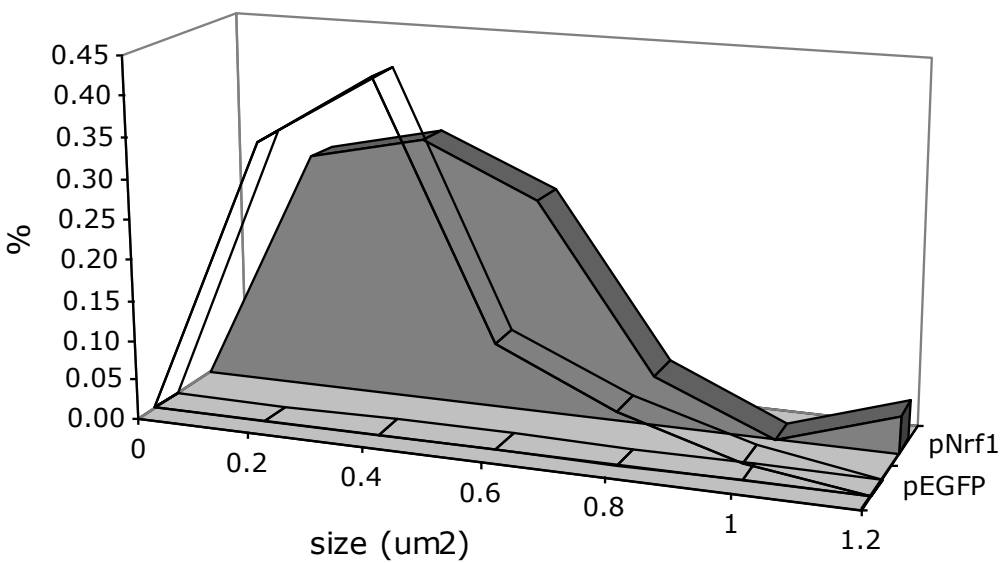

Fig. 6. Size distribution mitochondria of pNrf1 and pEGFP transfected 3T3-L1 adipocytes. Mitochondrial size was quantified using Image $\mathrm{J}$ analysis of electron microscopy images 
Gene expression analysis indicated increased apoptosis in Nrf1 overexpressing cells. The activity of caspase $3 / 7$ was measured, which are the main executors of apoptosis, to functionally test this at day 8 of differentiation. Caspase $3 / 7$ activity was found significantly increased $(p<0.05 ; n=3)$ in pNrf1 overexpressing cells $(1.40 \pm 0.13)$ compared with pEGFP transfected cells $(1.00 \pm 0.05)$.

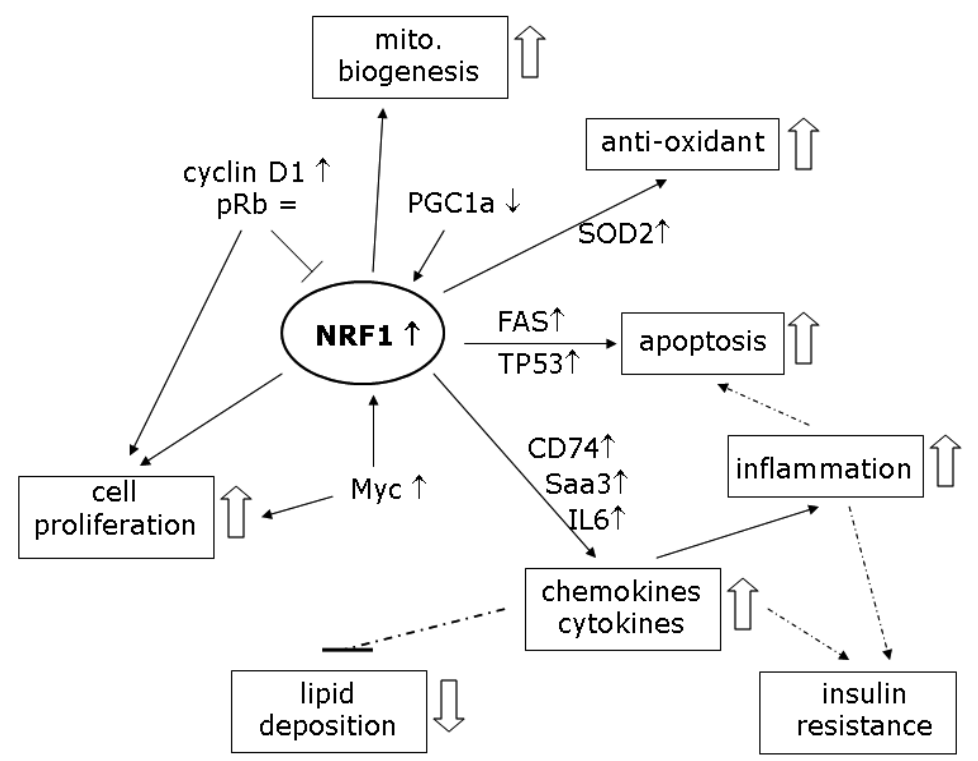

Fig. 7. Processes altered as a result of Nrf1 overexpression in 3T3-L1 adipocytes.

Solid lines indicate directly stimulated processes, and dashed lines indicate secondary effects of NRF1. Gene symbols represent genes involved in the implicated processes. The change in gene expression induced by Nrf1-overexpressing is indicated with arrows; increased expression $(\uparrow)$, decreased expression $(\downarrow)$, or no change $(=)$.

\section{Discussion}

In this study we showed that continuous overexpression of Nrf1 during adipogenesis mainly stimulates cell proliferation, chemokine activation and apoptosis (Fig. 7). With regard to metabolism-related processes, besides increased mitochondrial density, no effects on energy metabolism were seen. We demonstrated that long-term changes can drastically differ from short-term effects. Long-term Nrf1 overexpression mimics insulin-resistant adipocytes in contrast to the short-term insulinsensitizing effect. 
Mitochondrial density, ATP production and lipid handling capacity in Nrf1-overexpressing 3T3-L1 adipocytes

Adipogenesis is characterized by increased lipid deposition and triglyceride synthesis, but, Nrf1-overexpressing 3T3-L1 cells displayed less and smaller lipid droplets than control pEGFP-transfected cells at day 8 of differentiation. Reduced mitochondrial function results in increased lipid droplet size [29], whereas, Nrf1 overexpression has been implicated to enhance mitochondrial function [15]. Therefore, we hypothesized that increased lipid oxidation might be responsible for the reduced lipid deposition in pNrf1 overexpressing 3T3-L1 cells. However, our results showed that increased mitochondrial density was not accompanied by increased ATP production, indicating that Nrf1-induced mitochondrial biogenesis is not sufficient to increase mitochondrial function, as also observed by Baar et al. [30]. In line with this, no changes on gene expression level were observed in pathways related to energy metabolism, indicating that the reduced lipid content was not a result of increased lipid oxidation. Expression of a number of genes involved in triglyceride synthesis, lipid transport and packaging were decreased in Nrf1 overexpressing cells, e.g. fat specific protein 27 (Fsp27), stearoyl-Coenzyme A desaturase 1 (Scd1), Fatty acid binding protein 4 (Fabp4) and cluster of differentiation 36 (Cd36). Therefore, reduced lipid deposition is most likely the result of reduced fatty acid synthesis [31], and increased lipolysis, as seen with knock-out of genes involved in lipid packaging, e.g. perilipin (Plin) or Fsp27 [32, 33].

Since lipid accumulation is a key feature of late adipogenesis and a frequently used parameter to analyze adipogenic capacity, we wanted to test if the reduced lipid content seen in Nrf1-overexpressing cells could be the result of reduced differentiation capacity. Gene expression analysis of two key adipogenic transcription factors, PPARY and C/EBPa, during differentiation (Fig. 3) indicated that Nrf1 overexpression had no significant effect on early adipogenesis. In addition, expression of Adipophilin (Adfp), which is a constituent of the globule surface and a characteristic of late differentiation and was increased as well in Nrf1overexpressing cells, and inhibitors of adipogenesis like Delta like kinase (DIk) and GATA binding protein 2 (Gata2) were decreased in Nrf1overexpressing cells. This excludes reduced differentiation as a possible explanation. 
Nrf1 induced cell proliferation

A number of genes up-regulated by Nrf1 contained a bZIP transcription factor binding site and were involved in a variety of processes, including proliferation and cell-cycle regulation, apoptosis and chemokine/cytokine signaling. These results are supported by increased expression of a number of genes involved in cell-cycle regulation, including $\mathrm{CMyc}_{\mathrm{C}} \mathrm{Cyclin}$ D1 (Ccnd1) and Ccnd3, which are important regulators of cell proliferation and adipogenesis $[34,35]$. In addition, $C / E B P \beta$ and $C / E B P \delta$, two bZIP containing transcription factors involved in clonal expansion $[36,37]$, were also increased at day 8 by Nrf1 overexpression. Mitochondria induced cell-cycle regulation has previously been shown in mouse embryonic fibroblast treated with mitochondrial inhibitors, which resulted in either cell-cycle arrest, repair or cell-death [38]. Furthermore, the role of Nrf1 in cell-cycle progression has previously been established by Cam et al. [18], and may explain the embryonic lethality in $\mathrm{Nrf1}^{-/-}$mice [39]. Overexpression of $\mathrm{Nrf1}$ may cause inappropriate proliferation, which is also a hallmark of cancer cells. In line with this, Nrf1 has been previously associated as one of the transcription factors involved in breast cancer malignant progression [40].

\section{Nrf1 induces apoptosis}

Gene expression profiling showed that a number of pathways involved in apoptosis were significantly changed in Nrf1-overexpressing adipocytes. This was confirmed by an increased caspase 3/7 activity. Apoptosis in Nrf1-overexpression adipocytes appeared to be a result of Nrf1 induced Fas expression, and was possibly further stimulated by chemokine activation and altered adipokine expression. Since the TNF receptor superfamily member 6 (Fas) has a bZIP binding domain, the increased expression of the Fas-mediated apoptosis signaling is likely a direct result of Nrf1 overexpression. In addition to TNFa induced Fas, increased inflammation-related adipokine expression in Nrf1 overexpressing cells may also contribute to increased apoptosis. Furthermore, Nrf1 also induced a number of genes involved in anti-oxidant defense, e.g. superoxide dismutase 2 ( $\operatorname{Sod} 2)$. Although this feature was not clearly represented in the pathway analysis, some key anti-oxidant genes were strongly increased in Nrf1 overexpressing cells, e.g. Sod2, Ceruloplasmin $(C p)$, and Nuclear factor erythroid 2-related factor 1 (Nfe2/1). Excessive Sod2 expression could result in oxidative stress 


\section{Chapter 6}

hypersensitivity and stimulate apoptosis [41], but the relation between Nrf1 and oxidative stress response requires further studying. Despite increased $C M y c$ expression, $B C / 2$ expression was unchanged and mitochondria were not irregularly shaped in Nrf1-overexpressing cells, therefore we do not expect mitochondria dependent induction of apoptosis, as seen by Morrish et al. [35]. Although we can only speculate, since we did not measure cytochrome $c$ or mitochondrial membrane potential. Possibly, we did not observe a similar effect in our Nrf1-overexpressing adipocytes because our cells were not serumdepleted. In line with their results, Nrf1-overexpression in 3T3-L1 cells showed a trend of enlarged and more electron dense mitochondria (Fig. $5)$, likely due to increased import of mitochondrial proteins.

Long-term Nrf1 overexpression induces inflammation and insulinresistant adipokine expression profile

Our data indicate that inflammation in Nrf1-overexpressing cells is the result of both primary Nrf1 targets, as well as triggers of inflammation induced secondary to Nrf1 overexpression. Nrf1 increased a number of cytokines, e.g. cluster of differentiation 47 and 74 ( $C d 47$ and Cd74), which attract macrophages [42], and chemokines like C-X-C motif chemokine $(\mathrm{CxCl}) 2$ and 5, chemokine ligand $(\mathrm{CCl}) 2$ and 7. In addition, a number of adipokines, were amongst the strongest Nrf1 induced genes at day 8 , but some were also increased at earlier time-points (Fig. 4). Strong and immediate induction of e.g. Saa3 and $C p$ indicate that they are direct targets of $\mathrm{Nrf1}$, and that their induction is not a consequence of increased cell density and/or altered adipokine expression pattern later in differentiation, as seen with e.g. Hp and Adipoq. In our study, adiponectin was strongly reduced at day 6 and 8 of differentiation, despite increased mtDNA copy number and normal ATP production in Nrf1-overexpressing cells. These results oppose an earlier study which showed that two days overexpression of Nrf1 resulted in increased mtDNA content and increased adiponectin expression in 3T3-L1 adipocytes [15]. Based on these findings we hypothesize that reduced adiponectin expression is probably not solely a result of reduced mitochondrial content and/or functioning, but from altered adipokine signaling, e.g. increased TNFa and IL6. Taken together, our results indicate that Nrf1 can induce inflammation in adipocytes directly, which likely affects lipid handling in adipocytes [43]. Nrf1-overexpression probably triggers a pro-survival mechanism, which is also seen after 
exposure of cells to the endotoxin lipopolysaccharide (LPS) [44]. We do not expect that our results are an artifact from transfection induced LPScontamination, since inflammation is predominantly present in late adipogenesis and key adipogenic transcription factors are not decreased, and early adipogenesis is not inhibited [45].

In summary, our results demonstrate that Nrf1 is a key regulator of a broad variety of processes (Fig. 7). Some properties of Nrf1 are beneficial to combat T2DM and lipodystrophy, like stimulation mitochondrial biogenesis, anti-oxidant and cell proliferation, while others, e.g. stimulation of inflammation and apoptosis, worsen insulin resistance. These data indicate that direct Nrf1 overexpression is not recommended, but specific post-transcriptional regulation, or activation of Nrf1 targets could be beneficial for increasing adipocyte tissue mass thereby reducing lipid deposition in peripheral tissues [46, 47]. In addition, our results show that despite promising short-term Nrf1 overexpression, long-term secondary effects can drastically differ.

\section{Acknowledgements}

We would like to thank $H$. Duimel for his assistance in the electron microscopy studies. This research was funded by the Dutch diabetes foundation (DFN 2004.00.040) and the Kerry foundation. 


\section{References}

1. Wild, S., et al., Global prevalence of diabetes: estimates for the year 2000 and projections for 2030. Diabetes Care, 2004. 27(5): p. 1047-53.

2. Smyth, S. and A. Heron, Diabetes and obesity: the twin epidemics. Nat Med, 2006. 12(1): p. 75-80.

3. Guilherme, A., et al., Adipocyte dysfunctions linking obesity to insulin resistance and type 2 diabetes. Nat Rev Mol Cell Biol, 2008. 9(5): p. 367-77.

4. Dahlman, I., et al., Downregulation of electron transport chain genes in visceral adipose tissue in type 2 diabetes independent of obesity and possibly involving tumor necrosis factor-alpha. Diabetes, 2006. 55(6): p. 1792-9.

5. Mootha, V.K., et al., PGC-1alpha-responsive genes involved in oxidative phosphorylation are coordinately downregulated in human diabetes. Nat Genet, 2003. 34(3): p. 267-73.

6. Lowell, B.B. and G.I. Shulman, Mitochondrial dysfunction and type 2 diabetes. Science, 2005. 307(5708): p. 384-7.

7. Mallon, P.W., et al., In vivo, nucleoside reverse-transcriptase inhibitors alter expression of both mitochondrial and lipid metabolism genes in the absence of depletion of mitochondrial DNA. J Infect Dis, 2005. 191(10): p. 1686-96.

8. Munoz-Malaga, A., et al., Lipomatosis, proximal myopathy, and the mitochondrial 8344 mutation. A lipid storage myopathy? Muscle Nerve, 2000. 23(4): p. 538-42.

9. Choo, H.J., et al., Mitochondria are impaired in the adipocytes of type 2 diabetic mice. Diabetologia, 2006. 49(4): p. 784-91.

10. Patti, M.E., et al., Coordinated reduction of genes of oxidative metabolism in humans with insulin resistance and diabetes: Potential role of PGC1 and NRF1. Proc Natl Acad Sci U S A, 2003. 100(14): p. 8466-71.

11. Arany, Z., PGC-1 coactivators and skeletal muscle adaptations in health and disease. Curr Opin Genet Dev, 2008. 18(5): p. 426-34.

12. Bogacka, I., et al., Pioglitazone induces mitochondrial biogenesis in human subcutaneous adipose tissue in vivo. Diabetes, 2005. 54(5): p. 1392-9.

13. Lv, Q., et al., Effect of pioglitazone on visfatin expression in 3T3-L1 adipocytes and SD rats. Endocr Res, 2009. 34(4): p. 130-41.

14. Sugii, S., et al., PPARgamma activation in adipocytes is sufficient for systemic insulin sensitization. Proc Natl Acad Sci U S A, 2009. 106(52): p. 22504-9.

15. Koh, E.H., et al., Essential role of mitochondrial function in adiponectin synthesis in adipocytes. Diabetes, 2007. 56(12): p. 2973-81.

16. Timmons, J.A., et al., Expression profiling following local muscle inactivity in humans provides new perspective on diabetes-related genes. Genomics, 2006. 87(1): p. $165-72$.

17. Gerhart-Hines, Z., et al., Metabolic control of muscle mitochondrial function and fatty acid oxidation through SIRT1/PGC-1alpha. Embo J, 2007. 26(7): p. 191323.

18. Cam, H., et al., A common set of gene regulatory networks links metabolism and growth inhibition. Mol Cell, 2004. 16(3): p. 399-411.

19. Ho Sui, S.J., et al., oPOSSUM: identification of over-represented transcription factor binding sites in co-expressed genes. Nucleic Acids Res, 2005. 33(10): p. 3154-64. 
20. Ihaka, R. and R. Gentleman, "R: a language for data analysis and graphics". Journal of Computational Graphics and Statistics, 1996. 5(3): p. 299-314.

21. Lindsey, J., Models for repeated measurements, 2nd edition. 1999, Oxford: Oxford University Press. 536.

22. Dai, M., et al., Evolving gene/transcript definitions significantly alter the interpretation of GeneChip data. Nucleic Acids Res, 2005. 33(20): p. e175.

23. http://brainarray.mbni.med.umich.edu. cited; chip description file].

Available from: http://brainarray.mbni.med.umich.edu.

24. Akaike, H. Information theory and an extension of the maximum likelihood principle. in Second International Symposium on Inference Theory. 1973. Budapest: Akadémiai Kiadó.

25. Salomonis, N., et al., GenMAPP 2: new features and resources for pathway analysis. BMC Bioinformatics, 2007. 8: p. 217.

26. Doniger, S.W., et al., MAPPFinder: using Gene Ontology and GenMAPP to create a global gene-expression profile from microarray data. Genome Biol, 2003. 4(1): p. R7.

27. Dennis, G., Jr., et al., DAVID: Database for Annotation, Visualization, and Integrated Discovery. Genome Biol, 2003. 4(5): p. P3.

28. Van Tienen, F.H. Supplementary material thesis. 2010 [cited; Available from: http://www.personeel.unimaas.nl/florence-vantienen/.

29. Vankoningsloo, S., et al., Mitochondrial dysfunction induces triglyceride accumulation in 3T3-L1 cells: role of fatty acid beta-oxidation and glucose. J Lipid Res, 2005. 46(6): p. 1133-49.

30. Baar, K., et al., Skeletal muscle overexpression of nuclear respiratory factor 1 increases glucose transport capacity. Faseb J, 2003. 17(12): p. 1666-73.

31. Rossmeisl, M., et al., Decreased fatty acid synthesis due to mitochondrial uncoupling in adipose tissue. Faseb J, 2000. 14(12): p. 1793-800.

32. Nishino, N., et al., FSP27 contributes to efficient energy storage in murine white adipocytes by promoting the formation of unilocular lipid droplets. J Clin Invest, 2008. 118(8): p. 2808-21.

33. Toh, S.Y., et al., Up-regulation of mitochondrial activity and acquirement of brown adipose tissue-like property in the white adipose tissue of fsp27 deficient mice. PLoS One, 2008. 3(8): p. e2890.

34. Fox, K.E., et al., Regulation of cyclin D1 and Wnt10b gene expression by CAMPresponsive element-binding protein during early adipogenesis involves differential promoter methylation. J Biol Chem, 2008. 283(50): p. 35096-105.

35. Morrish, F., C. Giedt, and D. Hockenbery, c-MYC apoptotic function is mediated by NRF-1 target genes. Genes Dev, 2003. 17(2): p. 240-55.

36. Tang, Q.Q., T.C. Otto, and M.D. Lane, CCAAT/enhancer-binding protein beta is required for mitotic clonal expansion during adipogenesis. Proc Natl Acad Sci U S A, 2003. 100(3): p. 850-5.

37. Narayanan, $\mathrm{K}$, et al., The CCAAT enhancer-binding protein (C/EBP)beta and Nrf1 interact to regulate dentin sialophosphoprotein (DSPP) gene expression during odontoblast differentiation. J Biol Chem, 2004. 279(44): p. 45423-32.

38. Kulawiec, M., V. Ayyasamy, and K.K. Singh, p53 regulates mtDNA copy number and mitocheckpoint pathway. J Carcinog, 2009. 8: p. 8. 


\section{Chapter 6}

39. Huo, L. and R.C. Scarpulla, Mitochondrial DNA instability and peri-implantation lethality associated with targeted disruption of nuclear respiratory factor 1 in mice. Mol Cell Biol, 2001. 21(2): p. 644-54.

40. Niida, A., et al., Integrative bioinformatics analysis of transcriptional regulatory programs in breast cancer cells. BMC Bioinformatics, 2008. 9: p. 404.

41. Kowald, A., H. Lehrach, and E. Klipp, Alternative pathways as mechanism for the negative effects associated with overexpression of superoxide dismutase. J Theor Biol, 2006. 238(4): p. 828-40.

42. Leng, L., et al., MIF signal transduction initiated by binding to CD74. J Exp Med, 2003. 197(11): p. 1467-76.

43. Gustafson, B. and U. Smith, Cytokines promote Wht signaling and inflammation and impair the normal differentiation and lipid accumulation in 3T3-L1 preadipocytes. J Biol Chem, 2006. 281(14): p. 9507-16.

44. Suliman, H.B., et al., Lipopolysaccharide stimulates mitochondrial biogenesis via activation of nuclear respiratory factor-1. J Biol Chem, 2003. 278(42): p. 415108.

45. Chung, S., et al., Preadipocytes mediate lipopolysaccharide-induced inflammation and insulin resistance in primary cultures of newly differentiated human adipocytes. Endocrinology, 2006. 147(11): p. 5340-51.

46. Gugneja, S. and R.C. Scarpulla, Serine phosphorylation within a concise aminoterminal domain in nuclear respiratory factor 1 enhances DNA binding. J Biol Chem, 1997. 272(30): p. 18732-9.

47. Wang, C., et al., Cyclin D1 repression of nuclear respiratory factor 1 integrates nuclear DNA synthesis and mitochondrial function. Proc Natl Acad Sci U S A, 2006. 103(31): p. 11567-72. 


\section{Chapter 7}

General discussion 


\section{General discussion}

The rapidly emerging incidence of T2DM world-wide has resulted in the doubtful honor of T2DM being classified as an epidemic, just like obesity [1]. T2DM development is a consequence of a variety of environmental and genetic factors, like excessive intake of high calorie foods, low physical activity levels and genetic predisposition, but the primary cause of T2DM development is insulin resistance. This leads to excessive insulin production by pancreatic $\beta$-cells and reduced insulin-mediated glucose uptake. When excessive insulin production is terminated, blood glucose rises and T2DM develops. Since mitochondria are key organelles involved in glucose, lipid and amino acid metabolism, mitochondrial dysfunction has been hypothesized to underlie insulin resistance [2]. However, despite extensive studies, the role of mitochondrial function in T2DM etiology remains under debate. The aim of this thesis was to characterize the role of mitochondrial density, integrity and function in skeletal muscle tissue with the development of T2DM. Additional altered processes in T2DM in skeletal muscle and preadipocytes were further identified using global gene expression analysis. The function and role of two genes specifically reduced in T2DM were studied in vitro. In this general discussion, the findings and significance of the different studies will be merged and discussed in a broader perspective of the pathophysiology and future treatment options for T2DM.

\section{Reduced lipid handling capacity in T2DM}

\section{Adipose tissue}

Obesity, a major risk factor for T2DM development, is characterized by an imbalance between energy intake and energy expenditure. Although adipose tissue has been regarded in the past as an inert organ for storage of excess lipid, it is nowadays well recognized as an endocrine organ and accounts together with skeletal muscle tissue for $>90 \%$ of insulin-stimulated glucose uptake $[3,4]$. As adipose tissue is the primary storage site for lipids, obesity is characterized by an increased adipocyte number (hyperplasia) and volume (hypertrophy). Gene expression profiling showed that obesity was associated with higher adipocyte and preadipocyte inflammation $[5,6]$. Furthermore, sustained 
lipid-overload results in enlarged adipocytes, which triggers inflammation, lipolysis and insulin resistance. Therefore, adipose tissue is one of the key organs in T2DM etiology.

Enlarged adipocytes are found in T2DM patients and prediabetic individuals and appear to be a marker of insulin resistance in prediabetic subcutaneous adipose tissue, independent of BMI [7-9]. Adipocyte enlargement is also associated with increased inflammation and reduced insulin-responsiveness in obese and insulin resistant individuals $[10,11]$. In addition, reduced expression of key regulators of adipogenesis, e.g. PPARY and C/EBP $\beta$ has been observed in adipocytes of T2DM subjects [7]. It was, however, unknown if the reduction in adipogenic capacity was a direct result of macrophage infiltration and altered adipokine signaling from enlarged adipocytes, or rather an intrinsic change within T2DM preadipocytes. As shown in chapter 4, global gene expression analysis of preadipocytes from T2DM and ageand BMI-matched controls revealed that T2DM preadipocytes displayed an expression profile of reduced differentiation capacity. Since the cells were cultured, these changes reflected intrinsic changes of the preadipocytes, and were not due to signaling from other cell-types. Our data are supported by the data of Isakson et al., who observed a negative relation of preadipocyte differentiation with BMI and adipocyte enlargement, despite a positive correlation between BMI and number of preadipocytes [12]. Since adipocyte tissue mass does not expand during adulthood [13], the relevance of a reduced preadipocyte differentiation capacity in T2DM subjects might be questioned. However, the same study showed annual renewal of $\sim 10 \%$ of the adipocytes [13]. T2DMmediated inhibition of the preadipocyte differentiation capacity may affect this renewal, reducing the number of adipocytes and resulting in a further increase in adipocyte volume and/or adipocyte lipolysis. This may progress to an insulin resistant state. Inhibition of adipocyte formation has been proposed as a treatment option for obesity. However, in light of T2DM etiology, the formation of adipocytes should not be blocked, but rather stimulated. Evidence supporting this conclusion is derived from lipodystrophy patients who have a reduced adipocyte tissue mass, which is like adipocyte enlargement, associated with ectopic lipid deposition and insulin resistance [14]. 


\section{Chapter 7}

\section{Skeletal muscle lipid handling}

The ability of fatty acids to induce skeletal muscle insulin resistance has been demonstrated nearly 50 years ago by Randle et al. in 1963. They hypothesized that insulin resistance was the result of substrate competition between fatty acids and glucose [15]. This hypothesis was later revised by the recognition that fatty acids directly inhibit insulinstimulated glucose uptake, rather than glucose oxidation [16]. A positive relation between intramyocellular lipid (IMCL) content and insulin resistance has been demonstrated by a number of groups, and implies a causal role for IMCL in the development of insulin resistance $[17,18]$. However, highly insulin sensitive endurance-trained athletes also had a similar or even higher IMCL content when compared with obese and T2DM subjects. This makes IMCL unlikely as a causal factor and ubiquitous marker for development of insulin resistance [19]. Although no structural differences in lipid droplet size or distribution was seen between athletes and obese insulin resistant subjects, the fatty acid make-up of the lipid droplet may differ between groups. IMCL can contain a variety of fatty acids, e.g. triacylglycerol (TAG), diacylglycerols (DAGs), ceramides, and long-chain acyl CoAs (LCACoAs). Intramyocellular TAGs are regarded as neutral lipids that are surrounded by a phospholipid monolayer and lipid droplet coating proteins like ADRP. In contrast, fatty acid intermediates, like DAG, ceramides, and LCACoAs, are not packed and current evidence demonstrates that they impair insulin signaling by activating DAG-sensitive PKC mediated serine phosphorylation of IRS1 and by inhibiting phosphorylation and activation of PKB/Akt downstream of IRS1 [20, 21]. As shown in chapter 2, expression of the gene encoding the lipid storage droplet protein ADRP was reduced in long-standing T2DM patients, suggestive of increased formation of lipid intermediates and associated inhibition of insulin signaling. In accordance with this, ADRP expression has been shown to increase with weight loss in obese non-diabetic subjects and in obese diabetic subjects after metformin or troglitazone treatment [22]. In contrast, another study observed reduced expression of ADRP after rosiglitazone treatment of T2DM subjects, and ADRP expression correlated negatively with insulin mediated glucose uptake [23].

In conclusion, adipose tissue plays a fundamental role in the development of insulin resistance and T2DM. Reduced adipogenic potential and adipose tissue dysfunction increase the burden of fatty 
acids in liver and skeletal muscle, which play a key role in maintaining glucose homeostasis. In contrast to the initial assumption that IMCL content correlates with insulin resistance, the current hypothesis is that instead of TAG, distinct forms of lipid-intermediates, e.g. ceramides and DAG, are involved in the development of insulin resistance by impairing insulin-mediated glucose uptake.

\section{Mitochondria dysfunction is secondary to T2DM}

The role of mitochondria in energy generation from carbohydrates, lipids and proteins, and the development of diabetes in patients with a specific mutation in the mtDNA $(m .3243 A>G)$ led to the hypothesis that decreased mitochondrial function could play a key role in T2DM development. A number of alterations in skeletal muscle mitochondrial quality and quantity has been reported for T2DM patients, including decreased mitochondrial size and density, reflected by a lower mtDNA copy number and/or reduced citrate synthase (CS) activity [2, 24]. Other studies have failed to confirm these observations in T2DM patients [25-27]. In our study (chapter 2) we performed ex vivo analysis of mitochondrial density, complex I and IV activity, and assessed the maximal ATP production rate in vivo by Phosphocreatine (PCr) recovery rate, in both newly diagnosed and long-standing T2DM subjects [28]. In addition, we assessed mtDNA copy number, mtDNA integrity and screened for the diabetogenic m.3243A>G mutation. Global gene expression analysis was performed as an unbiased approach to identify pathogenic processes. These comprehensive data on different aspects of mitochondrial function are a major improvement to previous studies, which assessed only one or just a few aspects. Moreover, the use of both newly identified untreated and long-standing insulin-treated subjects allowed us to distinguish between causal processes in T2DM etiology and secondary alterations. We showed that decreased mitochondrial density and complex I activity are only observed in longstanding T2DM subjects, which demonstrates that mitochondrial dysfunction is not a prerequisite for the development of T2DM. Our hypothesis is further supported by the large ATP overproduction capacity of mitochondria of $\sim 150$ fold, which makes it unlikely that the reported reduction of $30 \%$ of maximal ATP production capacity in T2DM subjects 


\section{Chapter 7}

$[29,30]$ would directly result in insufficient ATP production [31]. This finding was corroborated by the enhanced mitochondrial function in diabetic Asians [32]. Furthermore, diabetes is absent in the majority of patients with a strongly reduced OXPHOS function, despite development of a wide range of neuromuscular symptoms. Mitochondrial function and density are influenced by many other factors, like obesity, physical inactivity and medication. Therefore, the reduced mitochondrial function seen by us in long-standing T2DM subjects may not be the sole result of T2DM development, but can also be attributed to other factors. Reduced physical activity level in long-standing T2DM subjects opposed to healthy age- and BMI-matched controls is an obvious factor. As shown in Table 1 of chapter 1 , differences in T2DM and control group characteristics exist, which may influence assessment of mitochondrial function as a result of diabetes development. Differences in medication among T2DM subjects can also influence mitochondrial function. Generally, PPARy activators are excluded, but a number of studies analyzed mitochondrial function in T2DM subjects treated with metformin. Metformin inhibits mitochondrial complex I and enhances anaerobic glucose metabolism. The reduced ATP production rate in these patients is most likely the result of metformin use and not of T2DM pathology [33, 34]. Likewise, our long-standing T2DM patients were also metformin treated, which may also have contributed to the reduced complex I activity in our study.

Apart from differences in subject characteristics, the methods used to assess mitochondrial function vary between studies. In general, in vivo ATP production rate is assessed by Phosphocreatine ( $\mathrm{PCr}$ ) recovery rate, or the maximal ATP production rate is determined at basal- and/or insulin-stimulated condition. Major drawback of these in vivo studies is the inability to quantify and correct for mitochondrial density when assessing OXPHOS function. EX vivo analysis of OXPHOS capacity is generally performed by respirometry, or analysis of the individual OXPHOS complexes. Ex vivo techniques do allow to correct for mitochondrial density, but can introduce differences as a result of the analysis procedure, for example when using isolated muscle fibers or permeabilized mitochondria. These methodological and subject dependent differences complicate the comparison and judgment of the studies and illustrate the necessity to use multiple, standardized 
techniques to assess mitochondrial function, and a well-characterized patient and matched control group.

The association between IMCL content and development of insulin resistance has been thought to result from reduced mitochondrial $\beta$ oxidation [35]. Our observations that newly diagnosed T2DM patients have normal mitochondrial density and function, an IMCL content similar to age- and BMI-matched controls [28], and a gene expression profile of increased fatty acid utilization as energy source further oppose against a causal role for mitochondrial dysfunction in T2DM (chapter 2). The observed increase in IMCL content and reduction in mitochondrial density and complex I function in our group of long-standing T2DM subjects strengthens this hypothesis. However, we cannot determine whether mitochondrial dysfunction causes increased IMCL deposition, or if it is the reverse. A number of observations suggest that increased fat oxidation is required for development of insulin resistance. Mice on a high-fat diet can develop insulin resistance despite a significant increase in mitochondrial content [36] and mice lacking $\beta$-oxidation promoting malonyl-CoA decarboxylase $(M C D)$ have an increased glucose oxidative capacity and resist diet induced obesity despite a high IMCL content [37]. Subsequent mitochondrial dysfunction, which is associated with T2DM progression, could also be the result from increased oxidative stress. This is a plausible hypothesis, as mitochondria are the major site for the production of oxygen radicals and fatty acids are a rich source of reducing equivalents. Consequently, a metabolic imbalance resulting from enhanced energy input combined with reduced requirement or capacity to produce ATP may stimulate the formation of reactive oxygen species. A growing body of evidence is supporting this hypothesis. Insulin resistant $K_{K A^{y}}$ mice required hyperlipidemia for increased ROS formation [38], and fatty acid oxidation opposed to glucose oxidation is associated with increased oxidative stress [39]. In addition, Abdul-Ghani et al. demonstrated a normal ATP producing capacity in T2DM skeletal muscle samples, but this was associated with twice as much reactive oxygen species (ROS) to produce the same amount of ATP as nondiabetic subjects [40].

Like common T2DM, the m.3243A>G mutation is also associated with an enhanced oxidative stress level, which may contribute to initiation and progression of mitochondrial diabetes [41]. Although diabetes as a 


\section{Chapter 7}

result of the m.3243A $>\mathrm{G}$ mutation belongs to the class of monogenic forms of diabetes, it mimics common T2DM by its adult onset and development of insulin resistance [42]. In contrast to other mtDNA mutations that are rarely implicated in development of diabetes, over $90 \%$ of m.3243A $>$ G carriers develop diabetes before the age of 70 with a mean age of onset of 38 years [43]. Studies using cybrids showed that a reduced ATP production was observed from a m.3243A>G mutation load $>70 \%$, while people with a much lower mutation percentage already develop diabetes [44]. The development of diabetes in m.3243A $>\mathrm{G}$ carriers is not associated with a further reduction in $\mathrm{PCr}$ recovery rate [45]. Furthermore, in our study (chapter 3), expression of OXPHOS-related genes was higher in the controls and young diabetic m.3243A $>$ G carriers with a high m.3243A>G mutation percentage than in the group containing young carriers with more severe neuromuscular disease and older mildly affected carriers with a lower mutation percentage and later onset diabetes. These data indicate that abolished ATP production is not the determining factor in the development of insulin resistance, but presumably altered lipid metabolism and increased ROS production play a role. This has been reported for the m.3243A $>$ G mutation, and does not occur in all carriers of a primary mitochondrial defect. It should be worthwhile to investigate the levels of ROS production in carriers of mtDNA mutations with and without diabetes. Development of diabetes and hearing-loss are currently the only characteristics of m.3243A>G that correlate with mutation percentage $[43,46]$. Studying pancreatic $\beta$-cells and skeletal muscle of mitochondrial diabetes patients will increase our understanding of insulin resistance and mitochondrial malfunctioning in common T2DM patients. Since they are not obese, there is no obesity effect nor altered adipokine signaling present. Development of disease characteristics appears to depend on multiple factors rather than solely on mutation percentage or haplogroups [47]. Current gene expression analysis showed that the alterations in skeletal muscle gene expression between two clusters of a heterogeneous group of $m .3243 A>G$ carriers did not purely reflect clinical presentation or muscle pathology. Since development of symptoms increase with age, aging is clearly involved, but other factors that play a role remain to be identified.

In conclusion, our data and an increasing number of other studies support mitochondrial dysfunction being a consequence rather than a 
causal factor in the development of insulin resistance and T2DM. Emerging evidence proposes a causal role for lipid induced inhibition of insulin signaling in the development of T2DM. Both, mitochondrial dysfunction and increased oxidative stress are mainly linked to overt diabetic state and not to the development of insulin resistance. Therefore, they are likely more involved in T2DM disease progression and the development of diabetes associated complications. In T2DM studies, mitochondrial dysfunction is based on their ATP production capacity. However, it is more likely that other more subtle changes in mitochondria play a role in T2DM pathophysiology, e.g. insufficient sequestering of fatty acids, excessive formation of ROS, and processes like cell proliferation, differentiation and apoptosis. Processes that may also be altered in m.3243A>G carriers and predispose to diabetes development. However, irrespective of the cause, a reduced mitochondrial function and density is apparent in skeletal muscle of T2DM patients and stimulation of mitochondrial OXPHOS function may improve the diabetic state.

\section{Improved mitochondrial function and glycemic control in T2DM patients}

Since obesity and T2DM originate from an imbalance between energy intake and energy expenditure, the most effective T2DM therapy is weight loss and increased physical activity. Exercise training is the first advice general practitioners give to newly identified T2DM subjects, and numerous studies have shown the beneficial effects of this approach on glycemic control and cardiovascular risk profile [48]. In contrast, longstanding T2DM with polyneuropathy are generally not advised to participate in exercise training, because of possible adverse side-effects. However, Praet et al. showed that a low-level interval exercise training program is feasible and safe for these complicated T2DM patients [49]. Interval training makes use of short-intervals of high intensity training followed by lower intensity recovery training, as opposed to traditional training programs of longer periods of submaximal exercise training. Interval training was shown to induce stronger improvements in cellular signaling processes involved in energy metabolism. In addition, the short-interval periods do not result in overload of the cardiovascular system, which makes it a safe therapy for T2DM patients with a high 


\section{Chapter 7}

cardiovascular risk profile [50]. In chapter 2, we show that one year of low-level interval exercise training increases mitochondrial function and content, and also induces the same compensatory mechanisms present in newly identified, untreated T2DM subjects (chapter 2, figure 3). Lowlevel exercise training increased expression of genes involved in fatty acid $\beta$-oxidation, Krebs cycle, and oxidative phosphorylation, which may reduce the burden of lipid intermediates on insulin signaling. Low-level exercise training also induced PGC1a expression, which is regarded as key regulator of mitochondrial biogenesis and functioning. Although the magnitude of PGC1a expression and upstream activators is intensity dependent [51], our results show that the low-level exercise protocol applied in our study is already sufficient to stimulate PGC1a.

Exercise training is associated with improved glycemic control and a reduction in the requirement of oral blood glucose lowering medication and/or exogenous insulin in most studies. The magnitude of improvement is quite variable, and depends on type, duration and intensity of the exercise training protocol $[52,53]$. As a guideline, T2DM subjects should have a minimum energy expenditure of $1000 \mathrm{kcal} /$ week, which equals $\sim 30 \mathrm{~min} /$ day of moderate-intensity physical activity on five days per week [54]. In addition, the loss of body fat as a result of exercise only or combined with dietary intake restriction plays a key role [55-58]. The low-level exercise training protocol applied in our study (chapter 2) with complex T2DM patients did not result in changes in fasting blood glucose or HbA1c levels. However, it stabilized the exogenous insulin requirements of the patients, which had increased with $0.8 \mathrm{U} /$ month over the three preceding years. This stabilization was a result of increased whole-body insulin sensitivity, as there was no increase in insulin production observed. Since exercise training mainly improves fat oxidative capacity, this stabilization may also be a result of decreasing hepatic glucose output, increasing muscle mass, increasing glycogen and intramuscular lipid storage capacity, and/or improving skeletal muscle perfusion. Since the low-level exercise training protocol used in our study (chapter 2), did not affect subjects' weight, we can conclude that the observed alterations are a result of increased energy expenditure and are not a consequence of reduced energy intake during the exercise training period. In addition to the exercise induced alterations, weight loss by restricting energy intake may further improve insulin sensitivity, and reduce fasting glucose and plasma free fatty 
acids [59]. In addition to skeletal muscle alterations, life-style interventions also target other tissues implicated in T2DM. For example, lipid loss may result in smaller adipocytes with a more insulin sensitive adipokine secretion profile and reduced lipolysis [60], and liver insulinsensitivity [61].

Our data show that T2DM patients with complications can benefit from exercise training. Although this cannot completely reverse the disease, it stabilizes exogenous insulin requirements and likely prevents disease progression. Moreover, exercise induced $\beta$-oxidation and triglyceride deposition was also seen in newly identified T2DM subjects. Exerciseinduced mitochondrial function would likely reverse the diabetic state in newly identified T2DM subjects. Unfortunately, despite a number of campaigns, the majority of people with a high risk for the development of T2DM or those that have already been diagnosed with T2DM do not engage in life-style changes to restrain their energy intake and increase their energy expenditure [57, 62]. Therefore, industry and academic research groups focus on development and medication to treat T2DM. The predominant therapeutic target in T2DM patients is glycemic control. Reduction of blood glucose levels to a near-normal range will prevent/delay disease progression and development of T2DM-associated complications [63]. Despite the large pathophysiological heterogeneity of T2DM, therapy is currently uniform for nearly all T2DM subjects, which obviously results in a high number of non-responders. Personalized treatment strategies, which takes phenotypic characteristics, socio-cultural factors, biomarker data, and genetic makeup into account should improve T2DM treatment and predict and prevent T2DM development. The last decade, genetic testing has improved very rapidly and has significantly improved treatment of monogenetic diabetes patients that were initially diagnosed T1DM or T2DM patients [64-66]. With the fast development of SNP detection systems and next-generation sequencing technology, using a persons' genetic profile to tailor treatment of diseases has become more realistic. The past years, genome wide association studies (GWAS) have provided us with information on a number of SNP loci associated with T2DM. However, these SNPs explained relatively little ( $6 \%)$ of T2DM heritability [67]. Although these SNPs might have a considerable impact on the population attributable risk, they are rarely useful for predicting risks in individual patients and for prevention. Their main contribution 


\section{Chapter 7}

will be the unraveling of the entire network of factors involved in T2DM. Moreover, GWAS only allows detection of common SNPs, but more rare SNPs with a moderate penetrance and larger functional effects cannot be picked-up. Next generation sequencing does allow detection of these rare, moderately penetrant SNPs, which may cumulatively have a more pronounced effect on development of T2DM and could probably explain a significant part of the heritability. Moreover, since prices of nextgeneration sequencing runs are decreasing, the capacity is increasing and the 1,000 genome sequencing initiative will provide a comprehensive catalogue of variants. Whole-genome sequencing will likely become a quick and valuable tool for identifying diabetes associated pathological variants in the future.

Complex diseases like T2DM depend on many different factors, which is a major challenge for personalized medicine. Although currently available SNP detection and next-generation sequencing techniques speed up the identification of variants, the assessment of the functional effect of a variant is a more tedious and laborious task. The amount of data and variants generated will be huge. For common SNPs it is known, that the functional effect can be small. It is expected that the stronger functional effects of the rare variants will make it easier to elucidate their role in pathogenesis. Other issues will certainly also dominate the discussions on next-generation sequencing leading to the personal genome. Privacy, legal and societal legislation issues will have to be overcome. The personal genome data will not only be usable for risk prediction or prevention. Drug metabolism is also largely genetically defined and pharmacogenetics or comparative-effectiveness research, which takes groups of patients into account, will be equally important to move towards personalized medicine. The identification of genetic variants, which differ between responders and non-responders of a treatment or rapid or slow metabolizers, will allow prediction of a clinical response. Polymorphisms in a number of genes have been identified that influence response to e.g. metformin, sulphonylureas and thiazolidinediones [68], but more studies are warranted to identify the complete set of risk alleles and their effect on enzyme or protein function. 


\section{In vitro characterization of genes implicated in T2DM pathophysiology}

Identification of therapeutic targets requires extensive knowledge of the function and the metabolic network the target gene is involved in. Putative targets often arise from comparative studies in the (affected) tissue of both patients and controls or from genome wide association studies, which allow detection of genetic associations with observable traits, e.g. diabetes, weight and blood pressure. Follow-up studies using human material are often not possible due to limited sample and/or difficult culture of primary cells. Therefore, model systems have been developed. The Zucker diabetic fatty (ZDF) rats, and the ob/ob and $d b / d b$ mice are most commonly used to mimic obesity and T2DM. In addition to animal models, (mouse) cell-lines are extensively used to identify the function of a gene and screen compounds, since they can still be reproducibly differentiated after a few passages. To assess the role of $W n t 5 b$, which was implicated in the adipogenesis process and had a reduced expression in T2DM preadipocytes (chapter 4), we analyzed its role during adipogenesis using mouse 3T3-L1 cells. As shown in chapter 5, expression of Wnt5b was, like its homolog Wnt5a, down-regulated upon induction of differentiation and both inhibited $\beta$ catenin dependent Wnt signaling at the initiation of adipogenesis [69]. In contrast to Wnt5a, Wnt5b stimulated PPARY. The down-regulation of $W n t 5 b$ in T2DM preadipocytes likely contributes to decreased adipogenic capacity by reduced stimulation of key adipogenic regulator PPARY. As described in chapter 6, we applied a similar experimental setup to identify the transcriptional targets and effect of Nrf1 overexpression during adipogenesis of mouse preadipocytes. NRF1 is regarded as key regulator of mitochondrial biogenesis, and expression of both NRF1 as its upstream activator PGC1a have been found reduced in T2DM [70]. Furthermore, PPARY agonists have been shown to increase insulin sensitivity and adiponectin expression in adipocytes in conjunction with increased mitochondrial content [71]. In agreement with this, two-day overexpression of $\mathrm{Nrf1}$ in adipocytes resulted in a significant increase in mtDNA content and increased adiponectin expression [72]. However, Nrf1 is not solely regulated by Pgc1a, and Nrf1 is also involved in other processes, e.g. cell proliferation and immune-response [73, 74]. As shown in chapter 6, Nrf1 overexpression during adipogenesis resulted 


\section{Chapter 7}

in increased mtDNA content, but it also induced processes linked to inflammation and cell proliferation, which were not seen after two-days Nrf1 overexpression [72]. This mimicked the gene expression profile of insulin resistant adipocytes with increased pro-inflammatory adipokine expression and reduced adiponectin expression. Our results indicated that Nrf1 linked metabolism to cell proliferation and shows the interaction and multiple routes of regulation on cell-level. The central role of Nrf1 explains the lack of consistent results obtained for NRF1 in PGC1a stimulated or deprived cells $[75,76]$. Identification of the exact signaling processes and branches downstream of Nrf1 is essential to identify more specific therapeutic targets to gain cell mass or mtDNA biogenesis, without triggering other Nrf1-induced processes. Our results further highlight the importance of identifying secondary targets in addition to the genes primary regulated by the putative therapeutic target. Although differences are apparent between human and mouse cells, significant gain in process-knowledge is obtained via in vitro analysis of genes and processes using murine cell-lines, demonstrating their value. Moreover, the use of unbiased microarray analysis allows the detection of unexpected relations and processes involved. The relevance of distinguishing primary and secondary processes and upand downstream targets is also exemplified by the TGF $\beta$ paradox in Marfan syndrome as well as cancer [77, 78]. Both enhanced and reduced TGF $\beta$ are implicated in these disorders, however, depending on the disease context, the effect of TGF $\beta$ therapy differs. To study the complex regulatory mechanisms, the usage of model systems, the unbiased screening of alterations and the step-by-step characterization of the complex regulatory networks involved are indispensible to develop and fine-tune effective therapeutic interventions.

\section{Conclusions}

This thesis provides a comprehensive overview of the mitochondrial and molecular alterations associated with different stages of T2DM development. Our approach bypassed a number of pitfalls of previous studies and showed that mitochondrial dysfunction is rather a consequence than a cause of T2DM, which can be improved by regular exercise training (chapter 2). Gene expression analysis provided a 
molecular explanation for the mitochondrial dysfunction observed in skeletal muscle of long-standing T2DM (chapter 2), but also identified the intrinsic molecular alterations that likely contribute to the reduced adipogenic potential of T2DM preadipocytes (chapter 4). Moreover, unsupervised clustering of gene expression data from a heterogeneous group of m.3243A>G carriers was applied as an unbiased approach to define patient clusters and subsequently identify differences between the clusters. Neither clinical phenotype nor skeletal muscle pathology could explain the gene expression differences between the clusters. It was striking that the relatively young patients with diabetes and high mutation load clustered with the controls, indicating that muscle might not be the first tissue revealing pathological processes for these patients. However, it is evident that the key discriminating factors for the entire group need to be identified by follow-up investigations, based on the differences in the processes observed (chapter $\mathbf{3}$ ). Although the relevance of gene expression analysis is sometimes doubted, our results show that much knowledge can be gained from gene expression analysis as it provides detailed insights in the underlying molecular mechanisms and reveals potential predictive markers and new targets for therapy. Subsequent in vitro characterization of two genes implicated in T2DM pathology showed that $W n t 5 b$ is a potent inducer of key adipogenic regulators (chapter 5), and implicated a key role for Nrf1 in a number of processes which were not all beneficial for treatment of T2DM (chapter 6). These results demonstrate the requirement to extent knowledge on the signaling cascades underlying the altered processes associated with T2DM, especially for selecting genes as targets for therapy. 


\section{References}

1. Smyth, S. and A. Heron, Diabetes and obesity: the twin epidemics. Nat Med, 2006. 12(1): p. 75-80.

2. Kelley, D.E., et al., Dysfunction of mitochondria in human skeletal muscle in type 2 diabetes. Diabetes, 2002. 51(10): p. 2944-50.

3. Reaven, G.M., Banting lecture 1988. Role of insulin resistance in human disease. Diabetes, 1988. 37(12): p. 1595-607.

4. Minokoshi, Y., C.R. Kahn, and B.B. Kahn, Tissue-specific ablation of the GLUT4 glucose transporter or the insulin receptor challenges assumptions about insulin action and glucose homeostasis. J Biol Chem, 2003. 278(36): p. 33609-12.

5. Lee, Y.H., et al., Microarray profiling of isolated abdominal subcutaneous adipocytes from obese vs non-obese Pima Indians: increased expression of inflammation-related genes. Diabetologia, 2005. 48(9): p. 1776-83.

6. Nair, S., et al., Increased expression of inflammation-related genes in cultured preadipocytes/stromal vascular cells from obese compared with non-obese Pima Indians. Diabetologia, 2005. 48(9): p. 1784-8.

7. Yang, X., et al., Evidence of impaired adipogenesis in insulin resistance. Biochem Biophys Res Commun, 2004. 317(4): p. 1045-51.

8. Arner, E., et al., Adipocyte turnover: relevance to human adipose tissue morphology. Diabetes. 59(1): p. 105-9.

9. Lundgren, M., et al., Fat cell enlargement is an independent marker of insulin resistance and 'hyperleptinaemia'. Diabetologia, 2007. 50(3): p. 625-33.

10. Hammarstedt, A., et al., Wnt-signaling is maintained and adipogenesis inhibited by TNFalpha but not MCP-1 and resistin. Biochem Biophys Res Commun, 2007. 357(3): p. 700-6.

11. Franck, N., et al., Insulin-induced GLUT4 translocation to the plasma membrane is blunted in large compared with small primary fat cells isolated from the same individual. Diabetologia, 2007. 50(8): p. 1716-22.

12. Isakson, P., et al., Impaired preadipocyte differentiation in human abdominal obesity: role of Wnt, tumor necrosis factor-alpha, and inflammation. Diabetes, 2009. 58(7): p. 1550-7.

13. Spalding, K.L., et al., Dynamics of fat cell turnover in humans. Nature, 2008. 453(7196): p. 783-7.

14. Mallon, P.W., et al., In vivo, nucleoside reverse-transcriptase inhibitors alter expression of both mitochondrial and lipid metabolism genes in the absence of depletion of mitochondrial DNA. J Infect Dis, 2005. 191(10): p. 1686-96.

15. Randle, P.J., et al., The glucose fatty-acid cycle. Its role in insulin sensitivity and the metabolic disturbances of diabetes mellitus. Lancet, 1963. 1(7285): p. 785-9.

16. Dresner, A., et al., Effects of free fatty acids on glucose transport and IRS-1associated phosphatidylinositol 3-kinase activity. J Clin Invest, 1999. 103(2): p. 253-9.

17. Anderwald, C., et al., Effects of insulin treatment in type 2 diabetic patients on intracellular lipid content in liver and skeletal muscle. Diabetes, 2002. 51(10): p. 3025-32.

18. Perseghin, G., et al., Metabolic defects in lean nondiabetic offspring of NIDDM parents: a cross-sectional study. Diabetes, 1997. 46(6): p. 1001-9.

19. van Loon, L.J., et al., Intramyocellular lipid content in type 2 diabetes patients compared with overweight sedentary men and highly trained endurance athletes. Am J Physiol Endocrinol Metab, 2004. 287(3): p. E558-65.

20. Holland, W.L., et al., Lipid mediators of insulin resistance. Nutr Rev, 2007. 65(6 Pt 2): p. S39-46.

21. Chibalin, A.V., et al., Downregulation of diacylglycerol kinase delta contributes to hyperglycemia-induced insulin resistance. Cell, 2008. 132(3): p. 375-86.

22. Phillips, S.A., et al., Adipocyte differentiation-related protein in human skeletal muscle: relationship to insulin sensitivity. Obes Res, 2005. 13(8): p. 1321-9. 
23. Minnaard, R., et al., Adipocyte differentiation-related protein and OXPAT in rat and human skeletal muscle: involvement in lipid accumulation and type 2 diabetes mellitus. J Clin Endocrinol Metab, 2009. 94(10): p. 4077-85.

24. Ortenblad, N., et al., Reduced insulin-mediated citrate synthase activity in cultured skeletal muscle cells from patients with type 2 diabetes: evidence for an intrinsic oxidative enzyme defect. Biochim Biophys Acta, 2005. 1741(1-2): p. 206-14.

25. Boushel, R., et al., Patients with type 2 diabetes have normal mitochondrial function in skeletal muscle. Diabetologia, 2007. 50(4): p. 790-6.

26. Ritov, V.B., et al., Deficiency of subsarcolemmal mitochondria in obesity and type 2 diabetes. Diabetes, 2005. 54(1): p. 8-14.

27. Asmann, Y.W., et al., Skeletal muscle mitochondrial functions, mitochondrial DNA copy numbers, and gene transcript profiles in type 2 diabetic and nondiabetic subjects at equal levels of low or high insulin and euglycemia. Diabetes, 2006. 55(12): p. 3309-19.

28. De Feyter, H.M., et al., Early or advanced stage type 2 diabetes is not accompanied by in vivo skeletal muscle mitochondrial dysfunction. Eur J Endocrinol, 2008. 158(5): p. 643-53.

29. Phielix, E., et al., Lower intrinsic ADP-stimulated mitochondrial respiration underlies in vivo mitochondrial dysfunction in muscle of male type 2 diabetic patients. Diabetes, 2008. 57(11): p. 2943-9.

30. Mogensen, M., et al., Mitochondrial respiration is decreased in skeletal muscle of patients with type 2 diabetes. Diabetes, 2007. 56(6): p. 1592-9.

31. Holloszy, J.O., Skeletal muscle "mitochondrial deficiency" does not mediate insulin resistance. Am J Clin Nutr, 2009. 89(1): p. 463S-6S.

32. Nair, K.S., et al., Asian Indians have enhanced skeletal muscle mitochondrial capacity to produce ATP in association with severe insulin resistance. Diabetes, 2008. 57(5): p. 1166-75.

33. Brunmair, B., et al., Thiazolidinediones, like metformin, inhibit respiratory complex I: a common mechanism contributing to their antidiabetic actions? Diabetes, 2004. 53(4): p. 1052-9.

34. Feinstein, D.L., et al., Receptor-independent actions of PPAR thiazolidinedione agonists: is mitochondrial function the key? Biochem Pharmacol, 2005. 70(2): p. 177-88.

35. Kelley, D.E. and L.J. Mandarino, Fuel selection in human skeletal muscle in insulin resistance: a reexamination. Diabetes, 2000. 49(5): p. 677-83.

36. Hancock, C.R., et al., High-fat diets cause insulin resistance despite an increase in muscle mitochondria. Proc Natl Acad Sci U S A, 2008. 105(22): p. 7815-20.

37. Koves, T.R., et al., Mitochondrial overload and incomplete fatty acid oxidation contribute to skeletal muscle insulin resistance. Cell Metab, 2008. 7(1): p. 45-56.

38. Bonnard, C., et al., Mitochondrial dysfunction results from oxidative stress in the skeletal muscle of diet-induced insulin-resistant mice. J Clin Invest, 2008. 118(2): p. 789-800.

39. Anderson, E.J., H. Yamazaki, and P.D. Neufer, Induction of endogenous uncoupling protein 3 suppresses mitochondrial oxidant emission during fatty acidsupported respiration. J Biol Chem, 2007. 282(43): p. 31257-66.

40. Abdul-Ghani, M.A., et al., Mitochondrial reactive oxygen species generation in obese non-diabetic and type 2 diabetic participants. Diabetologia, 2009. 52(4): p. 574-82.

41. Pang, C.Y., H.C. Lee, and Y.H. Wei, Enhanced oxidative damage in human cells harboring A3243G mutation of mitochondrial DNA: implication of oxidative stress in the pathogenesis of mitochondrial diabetes. Diabetes Res Clin Pract, 2001. 54 Suppl 2: p. S45-56.

42. Szendroedi, J., et al., Impaired mitochondrial function and insulin resistance of skeletal muscle in mitochondrial diabetes. Diabetes Care, 2009. 32(4): p. 677-9.

43. Maassen, J.A., et al., Mitochondrial diabetes: molecular mechanisms and clinical presentation. Diabetes, 2004. 53 Suppl 1: p. S103-9. 
44. van den Ouweland, J.M., et al., Functional and morphological abnormalities of mitochondria harbouring the tRNA(Leu)(UUR) mutation in mitochondrial DNA derived from patients with maternally inherited diabetes and deafness (MIDD) and progressive kidney disease. Diabetologia, 1999. 42(4): p. 485-92.

45. van Elderen, S.G., et al., Phosphorus-31 magnetic resonance spectroscopy of skeletal muscle in maternally inherited diabetes and deafness A3243G mitochondrial mutation carriers. J Magn Reson Imaging, 2009. 29(1): p. 127-31.

46. Chinnery, P.F., et al., The spectrum of hearing loss due to mitochondrial DNA defects. Brain, 2000. 123 ( Pt 1): p. 82-92.

47. Pierron, D., et al., New evidence of a mitochondrial genetic background paradox: impact of the $J$ haplogroup on the A3243G mutation. BMC Med Genet, 2008. 9: p. 41.

48. Marwick, T.H., et al., Exercise training for type 2 diabetes mellitus: impact on cardiovascular risk: a scientific statement from the American Heart Association. Circulation, 2009. 119(25): p. 3244-62.

49. Praet, S.F., et al., Long-standing, insulin-treated type 2 diabetes patients with complications respond well to short-term resistance and interval exercise training. Eur J Endocrinol, 2008. 158(2): p. 163-72.

50. Wisloff, U., et al., Superior cardiovascular effect of aerobic interval training versus moderate continuous training in heart failure patients: a randomized study. Circulation, 2007. 115(24): p. 3086-94.

51. Egan, B., et al., Exercise intensity-dependent regulation of PGC-1 \{alpha\} $m R N A$ abundance is associated with differential activation of upstream signalling kinases in human skeletal muscle. J Physiol.

52. Sigal, R.J., et al., Effects of aerobic training, resistance training, or both on glycemic control in type 2 diabetes: a randomized trial. Ann Intern Med, 2007. 147(6): p. 357-69.

53. Bweir, S., et al., Resistance exercise training lowers HbA1c more than aerobic training in adults with type 2 diabetes. Diabetol Metab Syndr, 2009. 1: p. 27.

54. Di Loreto, C., et al., Make your diabetic patients walk: long-term impact of different amounts of physical activity on type 2 diabetes. Diabetes Care, 2005. 28(6): p. $1295-302$.

55. Wycherley, T.P., et al., A High Protein Diet With Resistance Exercise Training Improves Weight Loss And Body Composition In Overweight And Obese Patients With Type 2 Diabetes. Diabetes Care.

56. Pi-Sunyer, X., et al., Reduction in weight and cardiovascular disease risk factors in individuals with type 2 diabetes: one-year results of the look AHEAD trial. Diabetes Care, 2007. 30(6): p. 1374-83.

57. Dunstan, D.W., et al., Home-based resistance training is not sufficient to maintain improved glycemic control following supervised training in older individuals with type 2 diabetes. Diabetes Care, 2005. 28(1): p. 3-9.

58. Torjesen, P.A., et al., Lifestyle changes may reverse development of the insulin resistance syndrome. The Oslo Diet and Exercise Study: a randomized trial. Diabetes Care, 1997. 20(1): p. 26-31.

59. Gray, R.E., et al., Effect of weight loss on muscle lipid content in morbidly obese subjects. Am J Physiol Endocrinol Metab, 2003. 284(4): p. E726-32.

60. Varady, K.A., et al., Degree of weight loss required to improve adipokine concentrations and decrease fat cell size in severely obese women. Metabolism, 2009. 58(8): p. 1096-101.

61. Vitola, B.E., et al., Weight loss reduces liver fat and improves hepatic and skeletal muscle insulin sensitivity in obese adolescents. Obesity (Silver Spring), 2009. 17(9): p. 1744-8.

62. Wisse, W., et al., Prescription of physical activity is not sufficient to change sedentary behavior and improve glycemic control in type 2 diabetes patients. Diabetes Res Clin Pract.

63. Intensive blood-glucose control with sulphonylureas or insulin compared with conventional treatment and risk of complications in patients with type 2 diabetes 
(UKPDS 33). UK Prospective Diabetes Study (UKPDS) Group. Lancet, 1998. 352(9131): p. 837-53.

64. McCarthy, M.I. and A.T. Hattersley, Learning from molecular genetics: novel insights arising from the definition of genes for monogenic and type 2 diabetes. Diabetes, 2008. 57(11): p. 2889-98.

65. Masia, R., et al., An ATP-binding mutation (G334D) in KCNJ11 is associated with a sulfonylurea-insensitive form of developmental delay, epilepsy, and neonatal diabetes. Diabetes, 2007. 56(2): p. 328-36.

66. Pearson, E.R., et al., Switching from insulin to oral sulfonylureas in patients with diabetes due to Kir6.2 mutations. N Engl J Med, 2006. 355(5): p. 467-77.

67. Manolio, T.A., et al., Finding the missing heritability of complex diseases. Nature, 2009. 461(7265): p. 747-53.

68. Avery, P., S.S. Mousa, and S.A. Mousa, Pharmacogenomics in type II diabetes mellitus management: Steps toward personalized medicine. Pharmacogenomics and Personalized Medicine, 2009(2): p. 79-91.

69. Nishizuka, M., et al., Wnt4 and Wnt5a promote adipocyte differentiation. FEBS Lett, 2008. 582(21-22): p. 3201-5.

70. Patti, M.E., et al., Coordinated reduction of genes of oxidative metabolism in humans with insulin resistance and diabetes: Potential role of PGC1 and NRF1. Proc Natl Acad Sci U S A, 2003. 100(14): p. 8466-71.

71. Choo, H.J., et al., Mitochondria are impaired in the adipocytes of type 2 diabetic mice. Diabetologia, 2006. 49(4): p. 784-91.

72. Koh, E.H., et al., Essential role of mitochondrial function in adiponectin synthesis in adipocytes. Diabetes, 2007. 56(12): p. 2973-81.

73. Cam, H., et al., A common set of gene regulatory networks links metabolism and growth inhibition. Mol Cell, 2004. 16(3): p. 399-411.

74. Chang, W.T. and A.M. Huang, Alpha-Pal/NRF-1 regulates the promoter of the human integrin-associated protein/CD47 gene. J Biol Chem, 2004. 279(15): p. 14542-50.

75. Gerhart-Hines, Z., et al., Metabolic control of muscle mitochondrial function and fatty acid oxidation through SIRT1/PGC-1alpha. Embo J, 2007. 26(7): p. 191323.

76. Timmons, J.A., et al., Expression profiling following local muscle inactivity in humans provides new perspective on diabetes-related genes. Genomics, 2006. 87(1): p. $165-72$.

77. Tian, M. and W.P. Schiemann, The TGF-beta paradox in human cancer: an update. Future Oncol, 2009. 5(2): p. 259-71.

78. Dietz, H.C., TGF-beta in the pathogenesis and prevention of disease: a matter of aneurysmic proportions. J Clin Invest. 120(2): p. 403-7. 



\section{Summary}

\section{Samenvatting}




\section{Summary}

\section{Summary}

Type 2 diabetes mellitus (T2DM) is a disorder of energy metabolism, characterized by insulin resistance and pancreatic $\beta$-cell failure. The incidence of T2DM is rapidly increasing and it is currently regarded as an epidemic, like obesity. Skeletal muscle and adipose tissue are two key organs involved in T2DM pathophysiology, since they account for $>90 \%$ of insulin-mediated glucose uptake. Mitochondria generate the majority of ATP from glucose, lipid and amino acid metabolism through the oxidative phosphorylation system (OXPHOS). Mitochondrial dysfunction was hypothesized to underlie insulin resistance and T2DM. However, despite extensive studies, the role of mitochondrial function in T2DM etiology remains under debate. In addition to a role in common T2DM, a mitochondrial defect can also give rise to a monogenetic form of diabetes. For example the mitochondrial DNA mutation at position 3243 of the tRNA ${ }^{\text {Leu }}$ gene nearly always leads to development of diabetes. Aim of this thesis was to perform a comprehensive study of the alterations in skeletal muscle and adipose tissue regarding mitochondrial function and global gene expression analysis of common T2DM patients (chapter $\mathbf{2 , 4}$ ) and characterize skeletal muscle gene expression alterations in mildly and severely affected m.3243A>G carriers (chapter 3). In addition, we characterized two relevant genes altered in T2DM further in vitro, namely $W n t 5 b$ and Nrf1 (chapter 5 and 6). Finally, chapter 7 discusses the relevance of our findings and concludes with directions for future research to extent understanding of energy metabolism disorders and development of therapeutic options.

Chapter $\mathbf{1}$ is a general introduction on T2DM pathophysiology, key organs involved, and the role of mitochondria in energy metabolism and T2DM. Key organs are muscle, adipose tissue, pancreas and liver, of which we studied adipose tissue and muscle in further detail (chapter 2-6). Data on the primary or secondary role for mitochondria in the development of T2DM are conflicting and possible explanations are discussed. This question has also been addressed experimentally in chapter 2. Skeletal muscle samples from newly diagnosed diabetic patients, long-standing insulin-treated T2DM patients and age- and BMImatched controls were used for a cross-sectional analysis of mitochondrial density, mitochondrial function, and global gene 
expression. A reduced mitochondrial activity of complex I, mitochondrial density reflected by citrate synthase activity and OXPHOS-related gene expression were apparent in long-standing T2DM patients, but not in newly identified diabetes subjects. Microarray analysis indicated reduced expression of genes involved in OXPHOS and Krebs cycle, confirming the presence of mitochondrial alterations in long-standing T2DM patients. Gene expression analysis of newly identified T2DM subjects indicated normal mitochondrial function, but an increased utilization of fatty acids as energy source, possibly as a rescue mechanism to avoid detrimental effects of increased lipid(-intermediates) on insulin signaling. Our data indicated that mitochondrial dysfunction is a consequence rather than a cause of T2DM development. Next, we studied if prolonged resistance type and endurance type exercise training could improve the energy capacity of T2DM patients. Subsequent analysis after one year of regular interval exercise training of long-standing T2DM subjects showed that exercise training is feasible and beneficial for T2DM patients with polyneuropathy. In vivo analysis of mitochondrial function indicated an improvement of $21 \pm 2 \%$, but also showed an improved blood lipid profile and stabilization of the exogenous insulin requirement. The reduced expression of genes involved in aerobic energy generation in T2DM subjects compared with controls, was reversed and increased in the long-standing T2DM subjects after one year of exercise. Also, $\beta$ oxidation genes were increased, suggesting a higher usage of fatty acids for energy generation as seen in newly diagnosed T2DM subjects. Our data indicates that mitochondrial dysfunction in long-standing T2DM patients can, at least partly, be reversed by prolonged endurance and resistance type exercise training.

In contrast to the secondary mitochondrial defects in patients with T2DM, the m.3243A $>\mathrm{G}$ mutation is a monogenetic cause of diabetes. Carriers of the m.3243A>G mutation nearly always develop diabetes, either as primary phenotype or as one of the phenotypes in a whole spectrum of clinical symptoms. Since there is no exact correlation between the m.3243A>G mutation percentage with the background mtDNA haplogroups and clinical manifestations, we performed gene expression profiling of muscle RNA from patients with varying levels of the m.3243A>G mutation and controls to unravel differences and similarities between patients. Unsupervised clustering of the gene 


\section{Summary}

expression data revealed which of the m.3243A>G carriers cluster together and which processes differ between the clusters (chapter $\mathbf{3}$ ). The young diabetic m.3243A>G carriers grouped together with healthy controls and the young carriers with more severe neuromuscular disease and older mildly affected m.3243A>G carriers clustered in a second group. Subsequent analysis of differently expressed genes and processes showed that aging and neuromuscular disease progression, but not development of diabetes in m.3243A>G carriers, was accompanied by reduced expression of genes involved in aerobic energy generation. In addition, an imbalance between cell proliferation and apoptosis was apparent in the young more severely affected and older mildly affected patients, most likely resulting in less functional muscle cells. Based on our observations we hypothesized that interventions aimed at improving mitochondrial function and stimulating cell regeneration could prevent or delay disease progression.

Adipose tissue is next to muscle another important tissue in T2DM pathophysiology. As T2DM is generally preceded by obesity, adipose tissue is considered the first affected tissue. Obesity induced adipocyte overloading causes adipocyte enlargement, which is associated with altered adipokine signaling, inflammation, insulin resistance and reduced adipogenic potential. We studied the intrinsic changes in T2DM preadipocytes compared with age- and BMI-matched controls. Our data (chapter 4) showed a decreased expression level of genes involved in differentiation, providing a molecular explanation for the reduced adipogenic potential in T2DM preadipocytes, which most likely contributes to ectopic fat depositioning. The preadipocytes were cultured and, therefore, the observed alterations are considered intrinsic alterations of T2DM preadipocytes and are not the result of signaling from other cell-types. Gaining insight in the different molecular mechanisms of adipogenesis may lead to the identification of targets for therapeutic interventions, aiming at enhancing adipocyte differentiation and metabolism to improve lipid storage capacity. This could reduce ectopic fat depositioning and enhance insulin sensitivity as well. One of the genes that had a reduced expression in T2DM preadipocytes was $W N T 5 b$, whereas its paralog WNT5a was unchanged. Chapter 5 describes the role of $W n t 5 b$ and $W n t 5 a$ during adipogenesis. Induction of adipogenesis requires inhibition of the $\beta$-catenin dependent and stimulation of the $\beta$-catenin independent Wnt signaling pathway, in 
which $W n t 5 a$ and $W n t 5 b$ are both involved. Gene expression and protein analysis during murine 3T3-L1 preadipocyte differentiation showed that both $W n t 5 a$ and $W n t 5 b$ inhibit $\beta$-catenin dependent Wnt signaling at the start of adipogenesis, and increased levels of $\beta$-catenin dependent Wnt3a inhibited adipogenesis. Furthermore, in contrast to Wnt5a, Wnt5b appears to be a potent enhancer of adipogenic capacity by stimulation of $P P A R Y$ and $a P 2$. Down-regulation of $W n t 5 b$ could therefore contribute to decreased adipogenesis observed in T2DM diabetic subjects. Another gene down-regulated by T2DM is Nrf1, which is the key regulator of mitochondrial biogenesis. In chapter 6, Nrf1 was overexpressed during adipogenesis to analyze the effect on mitochondrial density and function, and characterize its downstream transcriptional targets. Nrf1 overexpressing cells had an increased mtDNA copy number and reduced lipid content, which was not associated with an increased ATP production rate per cell. Using global gene expression analysis, we observed that Nrf1 overexpression stimulated cell proliferation, apoptosis and cytokine expression. Moreover, we observed that in contrast to the insulinsensitizing effect of short-term Nrf1 overexpression, prolonged Nrf1 overexpression induced an adipokine expression profile of insulin resistant adipocytes. Because of the wide range of transcriptional targets, post-transcriptional regulation of Nrf1, or stimulating specific downstream Nrf1 targets may be a more suitable and specific approach for treating adipose tissue defects, instead of directly stimulating Nrf1 expression. Chapter $\mathbf{7}$ discusses the relevance of our findings in the current timeframe, in which new genetic technologies, especially nextgeneration sequencing, provide new opportunities for the development of personalized medicine and a genome-wide in vitro characterization of genes and their roles. The chapter concludes with future research options to gain further insight in mitochondrial energy metabolism and T2DM pathophysiology and development of new treatment options and scenarios. 


\section{Samenvatting}

\section{Samenvatting}

Type 2 diabetes mellitus (T2DM) is een aandoening van het energie metabolisme die gekarakteriseerd wordt door insuline resistentie en onvoldoende insulineproductie door falende $\beta$-cellen in de alvleesklier. De incidentie van T2DM stijgt snel en wordt momenteel als een epidemie beschouwd, net als obesitas. Twee belangrijke organen betrokken bij de ontwikkeling van T2DM zijn skeletspier en vetweefsel, aangezien ze samen zorgen voor meer dan $90 \%$ van de insuline-gestimuleerde glucose opname. Bij verbranding van glucose, vetten en eiwitten wordt de meerderheid aan ATP (energie) gegenereerd door middel van het oxidatieve fosforylatie systeem (OXPHOS) in de mitochondriën. Verminderde mitochondriële functie werd gedacht ten grondslag te liggen aan de ontwikkeling van insuline resistentie en T2DM. Ondanks vele studies is de rol van de mitochondriën bij de ontwikkeling van T2DM echter nog steeds onduidelijk. Naast een rol bij de ontwikkeling van T2DM, kan een mitochondrieel defect ook leiden tot een monogenetische vorm van diabetes. Bijvoorbeeld de mitochondriële DNA mutatie op positie 3243 van het tRNA ${ }^{\text {Leu }}$ gen leidt in bijna alle gevallen tot ontwikkeling van diabetes. Doel van dit proefschrift was door middel van een uitvoerige studie de veranderingen m.b.t. mitochondriële functie en globale genexpressie analyse in skeletspier en vetweefsel van T2DM patiënten in kaart te brengen (hoofdstuk 2 en 4). Genexpressie analyse is tevens gebruikt om de genexpressie veranderingen in skeletspier van mild aangedane en ernstig aangedane dragers van de m.3243A $>$ G mutatie in kaart te brengen (hoofdstuk 3). Daarnaast hebben we twee genen betrokken bij de ontwikkeling van T2DM, namelijk $W n t 5 b$ en $N r f 1$, verder geanalyseerd in vitro (hoofdstuk 5 en 6). Tot slot, hoofdstuk $\mathbf{7}$ bediscussieert de relevantie van onze bevindingen en sluit af met conclusies en suggesties voor toekomstig onderzoek om het inzicht in aandoeningen van het energie metabolisme te verruimen en therapieën te ontwikkelen.

Hoofdstuk 1 geeft een algemene introductie over T2DM pathofysiologie, de belangrijke organen die hierbij betrokken zijn en de rol van de mitochondriën in het energie metabolisme en de veranderingen geassocieerd met de ontwikkeling van T2DM. Spier, vetweefsel, alvleesklier en de lever zijn belangrijke organen betrokken bij de ontwikkeling van T2DM, waarvan skeletspier en vetweefsel meer 
in detail besproken zullen worden (hoofdstuk 2-6). De huidige gegevens betreffende de primaire of secundaire rol van de mitochondriën bij de ontwikkeling van T2DM zijn erg tegenstrijdig. In hoofdstuk 1 worden mogelijke verklaringen hiervoor bediscussieerd, welke in hoofdstuk $\mathbf{2}$ experimenteel getest worden. Skeletspier monsters van nieuw gediagnosticeerde T2DM patiënten, langdurig insuline-behandelde T2DM patiënten en controles met vergelijkbare leeftijd en BMI zijn gebruikt om T2DM-gerelateerde veranderingen in de hoeveelheid mitochondriën, mitochondriële functie en globale genexpressie te analyseren. In tegenstelling tot nieuw gediagnosticeerde T2DM patiënten, had de groep langdurige T2DM patiënten een verlaagde activiteit van mitochondrieel complex I en een verlaagde hoeveelheid mitochondriën bepaald aan de hand van de hoeveelheid citraat synthase. Genexpressie analyse bevestigde de mitochondriële veranderingen in de groep langdurige T2DM patiënten, aangezien deze een verminderde expressie hadden van genen betrokken bij de citroenzuurcyclus en het OXPHOS systeem. Uit genexpressie analyse van de groep nieuw gediagnosticeerde T2DM patiënten bleek de mitochondriële functie in deze groep vergelijkbaar met de controle groep. In de nieuw gediagnosticeerde T2DM patiënten was er wel verhoogd gebruik van vetzuren als energiebron, mogelijk om schade aan de insuline signalering door ophoping van vetzuren te beperken. Uit onze data concluderen wij dat een verminderde mitochondriële functie een gevolg is van T2DM ontwikkeling, en dus niet de oorzakelijk is. Vervolgens hebben we gekeken of langdurige duur- en krachttraining de mitochondriële functie van langdurige T2DM patiënten zou kunnen verbeteren. Uit analyse van langdurige T2DM patiënten die 1 jaar interval bewegingstraining ondergingen bleek dat bewegingstraining haalbaar en gunstig is voor T2DM patiënten met polyneuropathie. De in vivo mitochondriële functie bleek met $21 \pm 2 \%$ verbeterd te zijn. Bovendien hadden ze een verbeterd lipiden profiel in bloed en was de benodigde hoeveelheid exogene insuline gestabiliseerd. De verlaagde expressie van genen betrokken bij aërobe energie productie in langdurige T2DM patiënten ten opzichte van controles was in deze patiënten verhoogd na een jaar training. Tevens was de expressie van genen betrokken bij de $\beta$-oxidatie toegenomen, wat mogelijk duidt op een verhoogd gebruik van vetzuren om energie te maken, vergelijkbaar met nieuw gediagnosticeerde T2DM patiënten. Uit onze data kunnen we 


\section{Samenvatting}

concluderen dat verminderde mitochondriële functie in langdurig T2DM patiënten gedeeltelijk kan worden verbeterd door langdurige duur- en krachttraining.

In tegenstelling tot de verminderde mitochondriële functie ten gevolge van T2DM ontwikkeling, veroorzaakt de m.3243A>G mutatie een monogenetische vorm van diabetes. Bijna alle dragers van de m.3243A>G mutatie ontwikkelen diabetes als primair fenotype of als een van de fenotypes in een heel spectrum van klinische symptomen. Aangezien er geen exacte correlatie bestaat tussen het m.3243A>G mutatie percentage en de mtDNA haplogroepen of met de klinische manifestaties, hebben we genexpressie analyse uitgevoerd van skeletspier RNA van m.3243A>G dragers met een verschillend mutatie percentage en controles. Ongecontroleerde clustering is toegepast om na te gaan welke m.3243A>G dragers op basis van hun genexpressie profiel samenclusterden en welke processen verschillend waren tussen de clusters (hoofdstuk 3). De jonge m.3243A>G dragers met milde symptomen en diabetes clusterden samen met de gezonde controles in cluster 1. De zwaarder aangedane m.3243A $>\mathrm{G}$ dragers en oudere mild aangedane $\mathrm{m} .3243 \mathrm{~A}>\mathrm{G}$ dragers clusterden samen in het tweede cluster. $\mathrm{Na}$ het analyseren van de differentieel tot expressie komende genen en processen in de twee clusters bleek dat progressie van het ziektebeeld vergezeld werd door een verminderde expressie van genen betrokken bij aërobe energie productie. Tevens was er in de zwaarder aangedane groep m.3243A>G dragers een onbalans zichtbaar tussen genen betrokken bij celproliferatie en apoptose, die waarschijnlijk leidt tot een verminderd aantal functionele spiercellen. Op basis van onze resultaten stellen we dat interventies gericht op het verbeteren van de mitochondriële functie en stimulering van celregeneratie, progressie van het ziektebeeld zou kunnen voorkomen of uitstellen.

Naast skeletspier is ook vetweefsel een belangrijk weefsel dat betrokken is bij T2DM pathofysiologie. Aangezien ontwikkeling van T2DM normaliter wordt voorafgegaan door obesitas, kan vetweefsel als het eerst aangedane weefsel worden beschouwd. Obesitas leidt tot overbelasting van de adipocyten (vetcellen) waardoor deze vergroot worden. Door deze vergroting verandert het het patroon aan stoffen, genaamd adipokines, die door de adipocyten geproduceerd worden. Hierdoor ontstaat er ontsteking, insuline resistentie en een verlaagde 
capaciteit van preadipocyten om te ontwikkelen tot adipocyt. In hoofdstuk 4 hebben we de intrinsieke veranderingen in preadipocyten van T2DM patiënten vergeleken met preadipocyten van controles van vergelijkbare leeftijd en BMI. Onze data duidt op een verlaagd expressie niveau van genen betrokken bij differentiatie. Dit geeft een moleculaire verklaring voor de gereduceerde differentiatiecapaciteit in preadipocyten van T2DM patiënten, die hoogstwaarschijnlijk bijdraagt aan vetdepositie in andere weefsels dan het vetweefsel. Aangezien de preadipocyten gekweekt zijn, kunnen we stellen dat de gevonden veranderingen intrinsieke veranderingen van de T2DM preadipocyten zijn en niet het resultaat van invloeden van andere celtypen. Meer inzicht verkrijgen in de veranderingen die optreden tijdens het adipogenese proces, kan mogelijk leiden tot de identificatie van therapeutische interventiepunten gericht op stimulering van de differentiatie en verbetering van de insuline gevoeligheid. Een van de genen met een verlaagde expressie in T2DM preadipocyten was Wnt5b, terwijl zijn paraloog Wnt5a onveranderd was. Hoofdstuk 5 beschrijft de rol van Wnt5b en Wnt5a tijdens het adipogenese proces. Inductie van het adipogenese proces wordt geremd door de $\beta$-cateïne afhankelijke Wnt signalering waartoe o.a. Wnt3a behoort. Genexpressie en eiwit analyse tijdens de differentiatie van muizen 3T3-L1 preadipocyten toonde aan dat Wnt5a en $W n t 5 b$ die behoren tot de $\beta$-cateïne onafhankelijke Wnt signalering het adipogenese proces stimuleren door de $\beta$-cateïne afhankelijke Wnt signalering te remmen. In tegenstelling tot Wnt5a, bleek Wnt5b overexpressie het adipogenese proces duidelijk te versterken door middel van stimulatie van $P P A R Y$ en $a P 2$. Verlaagde expressie van Wnt5b zou daarom kunnen bijdragen aan de verlaagde differentiatiecapaciteit in preadipocyten van T2DM patiënten. Een ander gen waarvan de expressie verlaagd is in T2DM patiënten is Nrf1, dat een sleutelrol speelt in het mitochondriële biogenese proces. In hoofdstuk 6 is het effect van Nrf1 overexpressie op de mitochondriële biogenese en functie tijdens adipogenese geanalyseerd en hebben we genen die door Nrf1 transcriptioneel gereguleerd worden in kaart gebracht. Cellen waarin Nrf1 tot overexpressie werd gebracht hadden een verhoogd mitochondrieel DNA kopie aantal en een verlaagde hoeveelheid lipiden die niet veroorzaakt werd door een verhoogde ATP productie per cel. Met behulp van globale genexpressie analyse konden de processen waarin Nrf1 een rol speelt worden geïdentificeerd, namelijk 


\section{Samenvatting}

celproliferatie, apoptose en cytokine expressie. Daarnaast hebben we geobserveerd dat langdurige $N r f 1$ overexpressie leidt tot de ontwikkeling van een adipokine expressie profiel van insulineresistente adipocyten. Deze observatie is tegengesteld aan de verhoogde insuline gevoeligheid die wordt waargenomen bij kortdurende Nrf1 overexpressie. Gezien het groot aantal door Nrf1 transcriptioneel gereguleerde genen lijkt directe stimulatie van Nrf1 niet de aan te raden. Post-transcriptionele regulatie van Nrf1 of specifieke activatie van een door Nrf1 geïnduceerd gen lijkt een geschiktere benadering voor de behandeling van defecten aan het vetweefsel.

In hoofdstuk 7 wordt de relevantie van onze bevindingen afgezet tegen het huidig tijdsbeeld waarin nieuwe technologieën, met name de "nextgeneration sequencing" technologie, nieuwe kansen bieden voor de ontwikkeling van gepersonaliseerde geneeskunde en genoombrede in vitro analyse van genen en hun functie. Dit hoofdstuk sluit af met mogelijke toekomstplannen om het mitochondriële energie metabolisme en de T2DM pathofysiologie verder te ontrafelen en behandelmethoden op te zetten. 


\section{Dankwoord}




\section{Dankwoord}

\section{Dankwoord}

Als je als AIO begint dan is het schrijven van een dankwoord nog ver weg, zelfs als je de thesis naar de beoordelingscommissie stuurt lijkt het nog steeds ver weg, maar nu is het dan zover, wat dus betekent dat $\mathrm{m}^{\prime} \mathrm{n}$ boekje nu echt klaar is!

Een promotieonderzoek doe je nooit alleen en daarom is het op $z^{\prime} n$ plaats om hen die hieraan bijgedragen hebben te bedanken. Allereerst wil ik natuurlijk mijn promotor Prof. Smeets bedanken. Beste Bert, na mijn stage bij popgen gaf je me de mogelijkheid om als AIO te startten. Bedankt voor die kans, de vrijheid die je me in het project gegeven hebt en de hulp wanneer nodig. Jij vertrouwde er altijd op dat alles goed zou komen en wist bij tegenslagen er toch altijd iets positiefs in te zien. Gelukkig kwam je aanstelling als professor juist voordat de thesis naar de beoordelingscommissie ging en kun je mijn promotor zijn. Mijn dank gaat verder uit naar mijn twee copromotores Dr. van der Kallen en Dr. de Coo. Beste Carla, toen in het $1^{\mathrm{e}}$ jaar het project niet echt wilde opschieten gaf jij me de mogelijkheid om te starten met genexpressie analyse in preadipocyten, welke naar mijn mening leuke resultaten hebben opgeleverd. Bedankt dat ik altijd bij je kon binnenvallen en ik zal proberen in de toekomst de discussies van artikelen korter te maken. Beste René, een (kinder)neuroloog en een onderzoek naar T2DM lijkt niet een voor de hand liggende combinatie, maar is voor dit project wel heel belangrijk geweest. Toen het verzamelen van biopten niet wilde lukken heb jij via verschillende wegen geprobeerd aan biopten te komen en is door jouw hulp toch alles op z'n pootjes terechtgekomen, heel erg bedankt hiervoor.

Voor de T2DM studie in skeletspier heb ik met plezier samengewerkt met Luc van Loon, Stephan Praet, Jeanine Prompers en Nicole van den Broek. Luc, bedankt voor je kritische opmerkingen en suggesties voor het manuscript. Stephan, bedankt voor alle hulp bij het uitzoeken van de enorme berg klinische gegevens die je verzameld had en Jeanine en Nicole bedankt voor het (her)analyzeren van de NMR data.

Natuurlijk wil ik mijn collega's bij Popgen/Clinical Genomics bedanken, te beginnen met de AIO-kamer. De "oude" garde, Bianca, Lars, Nicole, 
Ruben en Rudy, waar later An, Rita, Abishek en Auke aan toe zijn gevoegd. Bianca, ik ben net zo "goed" als jou in het vinden van de weg, maar het is ons toch goed gelukt om in Aussois te komen (in verhouding sneller dan van Rotterdam CS naar het Erasmus MC ;-), maar ja, we hebben wel gelachen. Lars en Rudy, bedankt voor de IT support op de AIO kamer en het lachen om jullie strakke opmerkingen. Lars, gaan we nog eens de Kennedymars lopen? Nicole, we hebben veel gemeen, onder andere dat we als enigen in de groep werktte we aan T2DM, al was het vanuit een hele andere invalshoek welke leidde tot leuke discussies, en een leuk tripje naar de EASD in Kopenhagen. Veel succes nog in Saarbrücken en bedankt dat je paranimf wilde zijn. An, met jou klikte het vrijwel meteen en vaak was een blik al voldoende om in lachen uit te barsten (soms tot ongenoegen van omstanders). Succes met de laatste loodjes en jij ook bedankt dat je paranifm wilde zijn in deze drukke tijden voor jou! Ruben, onze enige echte Hollander op de AIO kamer die net als ik anti-carnaval is, je hebt veel labervaring en had vaak handige tips, bedankt hiervoor en veel succes in de VS en met de afronding van je thesis. Rita, ons onderzoek had niet veel gemeen, maar je zuiders temprament bracht wel leven in de brouwerij (AIO kamer) en ik ga je portugees gebak wel missen. Abishek, you were only a short period with us, but I doubt that you're new PhD-room is as fun as ours, good luck with your PhD study. Auke, je staat nog aan het begin van je AIO-traject, veel succes met de zebravisjes. Trouwens, bevalt "mijn" bureau aan het raam?

Ook de andere collega's wil ik graag bedanken. Rosy, bedankt dat ik altijd bij je terecht kon als er iets geregeld moest worden of als ik niet wist bij wie ik moest zijn voor iets. Patrick, this project required a number of statistical analyses and we encountered a number of pitfalls. Many thanks for performing the statistical analyses again, again and again! Ton, Fons, Miroslav en Jo, bedankt voor alle hulp als de PC weer eens niet met mij wilde meewerken. Mike, ik vond het dagje uit wat we hebben georganiseerd erg goed gelukt, veel succes nog met je thesis. Erika, dank voor je hulp met de Tecan('t) scanner en de vele dingen daarnaast waar je me mee geholpen hebt. Bieke bedankt voor het runnen van de Affymetrix arrays en Iris voor de qPCR die je nog op de valreep hebt gedaan. Caroline, als stagiaire ben ik ben ik bij jou en Rudy begonnen en jij hebt me wegwijs gemaakt op het lab en in het runnen van arrays. Ellen, Marion, Sabine, Jos, Roselie, Rob, Frank, Liesbeth, Jim, Bart, Wanwisa, Aimee, Anja, Torik en Mary, allen bedankt voor de 


\section{Dankwoord}

gezellige tijd bij Popgen. Ook de stagiaires mag ik niet vergeten te bedanken, met name Ivo voor alle qPCRs die je in de laatste maanden voor me hebt gedaan. Ik wil ook de mensen van klinische genetica bedanken, met name Suzanne voor het uitzoeken van de klinische gegevens voor de MELAS studie en de "mito" mensen Alexandra, Chantal en Eveline en Debby. Ook de "mito" mensen uit Rotterdam mag ik niet vergeten: Kees, Wim en Alex bedankt! De buren van Moleculaire Genetica en Celbiolgie, de mensen van Interne geneeskunde en Humane Biologie, bedankt voor jullie adviezen en de mogelijkheid af en toe iets te lenen. Hilde bedankt voor je hulp met het Wnt5b manuscript. Ingrid, bedankt voor op de goeie momenten een praatje te komen maken en veel succes met de afronding van thesis!

Pap en mam, bedankt voor alles wat jullie voor me gedaan hebben, ook al was mijn onderzoek "chinees" voor jullie, jullie waren altijd geïnteresseerd en hebben me altijd gesteund en op alle manieren proberen te helpen. Mijn broer(tje) Bob, jij ook bedankt en veel succes met je bedrijf.

Bart, bedankt voor al je steun de afgelopen jaren. Je had er nooit problemen mee als ik 's avonds weer eens achter de laptop zat en ging vaak mee op zondagmiddag naar de universiteit om de cellen te "verzorgen", vanaf nu kunnen we op zondag iets anders gaan doen.

Florence 


\section{Curriculum}

vitae 


\section{Curriculum vitae}

\section{Curriculum vitae}

Florence van Tienen werd geboren op 21 augustus 1980 te Sittard. Van 1992 tot 1996 doorliep zij het Middelbaar Administratief Voorbereidend Onderwijs (MAVO) aan MAVO St. Jacobus te Born, waarna zij startte met de Middelbare Laboratorium Opleiding (MLO) richting Klinische Chemie en Hematologie bij Leeuwenborgh Opleidingen te Sittard. Na het behalen van haar MLO diploma in 2000, startte ze met de Hogere Laboratorium Opleiding (HLO) specialisatie Moleculaire Biologie aan de Hogeschool Utrecht te Utrecht. Haar afstudeerstage werd uitgevoerd bij de afdeling Moleculaire Genetica van het Nederlands Kanker Instituut te Amsterdam en ze behaalde in 2003 haar Bachelor Degree. Vervolgens startte ze in 2003 haar Master opleiding Biomedische Wetenschappen aan de Vrije Universiteit te Amsterdam. Om zich verder te ontwikkelen en kennis te maken met verschillende takken van de moleculaire biologie liep ze stage bij de afdeling Genetica van de VU en de afdeling Moleculaire Biologie van de Universiteit van Edinburgh (UK), waar ze epigenetische veranderingen in Petunia Hybrida en Drosophila Melanogaster bestudeerde. Vervolgens liep te haar laastste stage bij de Universiteit Maastricht. Bij de sectie Populatie Genetica van de afdeling Genetica en Celbiologie maakte ze kennis met genexpressie analyse en mitochondriële ziekten. Na het behalen van haar universitair diploma in augustus 2005 bleef ze bij Populatie Genetica en startte ze met haar promotietraject als Assistent in Opleiding (AIO). Het promotieonderzoek was gericht op Type 2 Diabetes Mellitus (T2DM) en had als doel om meer inzicht te krijgen in de rol van de mitochondriën en pathologische processen betrokken bij de ontwikkeling van T2DM. Sinds 1 november 2010 is ze als development expert werkzaam bij EmulTech te Eindhoven. 


\section{Publications}




\section{Publications}

\section{Publications}

F.H.J. van Tienen, H. Laeremans, C.J.H. van der Kallen, H.J.M. Smeets (2009). Wnt5b stimulates adipogenesis by activating PPARY, and inhibiting the beta-catenin dependent Wnt signaling pathway together with Wnt5a. Biochem Biophys Res Commun 387(1): 207-11.

F.H.J. van Tienen, P.J. Lindsey, C.J.H. van der Kallen, H.J. Smeets (2010). Prolonged Nrf1 overexpression triggers inflammation and insulin resistance in 3T3-L1 adipocytes. Journal of Cellular Biochemistry, In press.

F.H.J. van Tienen, C.J.H. van der Kallen, P.J. Lindsey, R.J. Wanders, M.M. van Greevenbroek, H.J.M. Smeets. Preadipocytes of Type 2 Diabetes subjects display an intrinsic gene expression profile of decreased differentiation capacity. International Journal of Obesity, Accepted.

F.H.J. van Tienen, S.F.E. Praet, H.M. de Feyter, N.M. van den Broek, P.J. Lindsey, K.G.C. Schoonderwoerd, I.F.M. de Coo, K. Nicolay, J.J. Prompers, H.J.M. Smeets, and L.J.C. van Loon, Skeletal muscle mitochondrial dysfunction is secondary to type 2 diabetes and can be improved by prolonged exercise training. Manuscript submitted

F.H.J. van Tienen, P.J. Lindsey, S.C.E.H. Salleveld, S.G. Frints, I.F.M. de Coo, V. Tiranti, H.J.M. Smeets, Gene expression profiling reveals differences in pathological processes in skeletal muscle of affected carriers of the m.3243A >G (MELAS) mutation. Manuscript in preparation. 


\section{Abstracts}

43nd annual meeting EASD 2007, Kopenhagen (DK)

Keystone symposia Molecular control of Adipogenesis 2008, Banff (CA)

F.H.J. van Tienen, C.H.J. van der Kallen, P.J. Lindsey, H.J.M. Smeets. Gene expression profiling identifies molecular pathways that cause decreased differentiation of preadipocytes in Type 2 diabetes patients. (poster presentation)

FEBS Mitochondria in Life, Death and Disease 2007, Aussois (FR)

F.H.J. van Tienen, R.G. van Eijsden, C.M.M. van den Burg, W. Sluiter, K. Schoonderwoerd, R.F.M. de Coo, P.J. Lindsey, H.J.M. Smeets. The role of OXPHOS deficiency in the relation between the mitochondrial A3243G mutation and the type 2 diabetes phenotype (poster presentation).

Genetica Retraite 2009, Kerkrade (NL)

F.H.J. van Tienen, H. Laeremans, C.J. van der Kallen, H.J. Smeets. Wnt5b stimulates adipogenesis by activating PPARgamma, and inhibiting the beta-catenin dependent Wnt signaling pathway together with Wnt5a. (oral presentation)

UMDF Mitochondrial Medicine symposium 2010, Scottsdale (US)

F.H.J. van Tienen, S.F.E. Praet, H.M. de Feyter, N.M. van den Broek, P.J. Lindsey, K.G.C. Schoonderwoerd, I.F.M. de Coo, K. Nicolay, J.J. Prompers, H.J.M. Smeets, and L.J.C. van Loon, Skeletal muscle mitochondrial dysfunction is secondary to type 2 diabetes and can be improved by prolonged exercise training. (oral presentation). 
Abbreviations 


\section{Abbreviations}

$\begin{array}{ll}\text { 1RM } & \text { 1 repetition maximum } \\ \text { AIC } & \text { Akaike Information Criterion } \\ \text { BMI } & \text { body mass index } \\ \text { CI } & \text { confidence interval } \\ \text { CS } & \text { citrate synthase } \\ \text { DAG } & \text { diacylglycerol } \\ \text { DAVID } & \text { Database for Annotation, Visualisation and Integrated } \\ & \text { Discovery } \\ \text { DEXA } & \text { dual-energy x-ray absorptiometry } \\ \text { ECM } & \text { extracellular matrix } \\ \text { EI } & \text { exogenous insulin } \\ \text { EM } & \text { electron microscopy } \\ \text { ESM } & \text { electronic supplemetary material } \\ \text { FFA } & \text { free fatty acids } \\ \text { GEenMAPP } & \text { Gene Map Annotator and Pathway Profiler } \\ \text { GEO } & \text { gene expression omnibus } \\ \text { GO } & \text { gene ontology } \\ \text { HIT } & \text { high-intensity interval exercise training } \\ \text { HSL } & \text { hormone sensitive lipase } \\ \text { IBMX } & \text { 3-isobutyl-1-methylxantine } \\ \text { IDDM } & \text { Insulin Dependent Diabetes Mellitus } \\ \text { IGT } & \text { impaired glucose tolerant } \\ \text { IMCL } & \text { intramyocellular lipid } \\ \text { MRS } & \text { magnetic resonance spectroscopy } \\ \text { mEDNA } & \text { mitochondrial DNA } \\ \text { nDNA } & \text { nuclear DNA } \\ \text { NEFA } & \text { non-esterified fatty acids } \\ \text { OGT } & \text { oral glucose tolerance test } \\ \text { OXPHOS } & \text { oxidative phosphorylation } \\ \text { PCr } & \text { Phosphocreatine } \\ \text { PRT } & \text { progressive resistance type exercise training } \\ \text { qPCR } & \text { quantitative PCR } \\ \text { ROS } & \text { reactive oxygen species } \\ \text { RRF } & \text { ragged red fibers } \\ \text { rRNA } & \text { ribosomal RNA } \\ \text { SD } & \text { standard deviation } \\ & \end{array}$


SE

SGBS

SVC

T1DM

T2DM

TG

tRNA

TZD

VO2peak

WHO

Wmax standard error

Simpson-Golabi-Behmel Syndrome stromal vascular cells

Type 1 Diabetes Mellitus

Type 2 Diabetes Mellitus

triglycerides

transfer RNA

thiazolidinediones

maximal oxygen uptake capacity

world health organisation

maximal workload capacity 